\title{
Serotonin and integrated brain-gut function in irritable bowel syndrome
}

Citation for published version (APA):

Kilkens, T. O. C. (2007). Serotonin and integrated brain-gut function in irritable bowel syndrome. [Doctoral Thesis, Maastricht University]. Universitaire Pers Maastricht. https://doi.org/10.26481/dis.20071207tk

Document status and date:

Published: 01/01/2007

DOI:

10.26481/dis.20071207tk

Document Version:

Publisher's PDF, also known as Version of record

\section{Please check the document version of this publication:}

- A submitted manuscript is the version of the article upon submission and before peer-review. There can be important differences between the submitted version and the official published version of record.

People interested in the research are advised to contact the author for the final version of the publication, or visit the DOI to the publisher's website.

- The final author version and the galley proof are versions of the publication after peer review.

- The final published version features the final layout of the paper including the volume, issue and page numbers.

Link to publication

\footnotetext{
General rights rights.

- You may freely distribute the URL identifying the publication in the public portal. please follow below link for the End User Agreement:

www.umlib.nl/taverne-license

Take down policy

If you believe that this document breaches copyright please contact us at:

repository@maastrichtuniversity.nl

providing details and we will investigate your claim.
}

Copyright and moral rights for the publications made accessible in the public portal are retained by the authors and/or other copyright owners and it is a condition of accessing publications that users recognise and abide by the legal requirements associated with these

- Users may download and print one copy of any publication from the public portal for the purpose of private study or research.

- You may not further distribute the material or use it for any profit-making activity or commercial gain

If the publication is distributed under the terms of Article $25 \mathrm{fa}$ of the Dutch Copyright Act, indicated by the "Taverne" license above, 
Serotonin and integrated brain-gut function in irritable bowel syndrome 
(C) T. Kilkens, Maastricht 2007

ISBN: 978-90-5278-678-0

Layout: Tiny Wouters

Cover: Tessa Kilkens

Production: Datawyse | Universitaire Pers Maastricht 


\title{
Serotonin and integrated brain-gut function in irritable bowel syndrome
}

\author{
Proefschrift \\ ter verkrijging van de graad van doctor \\ aan de Universiteit Maastricht, \\ op gezag van de Rector Magnificus, Prof. mr. G.P.M.F. Mols, \\ volgens het besluit van het College van Decanen, \\ in het openbaar te verdedigen \\ op vrijdag 7 december 2007 om 12.00 uur
}

door

Theresia Odilia Catharina Kilkens

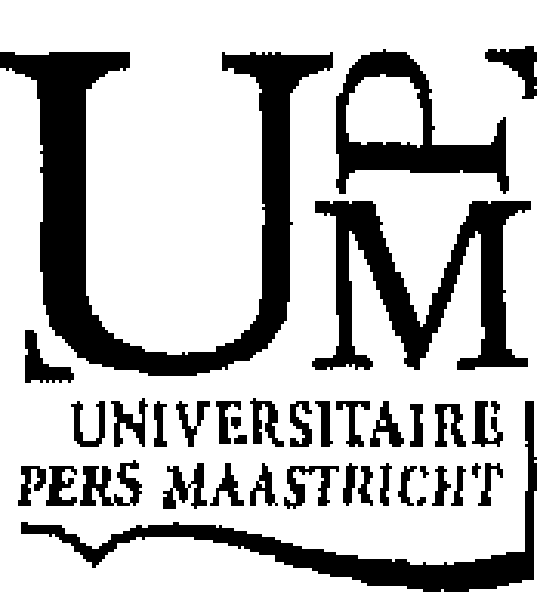




\section{Promotor}

Prof. dr. R-J. M. Brummer

\section{Copromotores}

Dr. A. Honig (St. Lucas Andreas ziekenhuis, Amsterdam)

Dr. M.A. van Nieuwenhoven

\section{Beoordelingscommissie}

Prof. dr. J. van Os (voorzitter)

Prof. dr. A.A.M. Masclee

Prof. dr. M. Schemann (TU, München)

Dr. M. Simrén (Sahigrenska University Hospital, Göteborg)

Prof. dr. R.W. Stockbrügger 


\section{Contents}

\section{Abbreviations}

Chapter $1 \quad$ General introduction 11

Chapter 2 Review article: serotonergic modulation and assessment of integrated brain-gut function in irritable bowel syndrome

Chapter 3 Systematic review: serotonergic modulators in the treatment of irritable bowel syndrome-influence on psychiatric and gastrointestinal symptoms

Chapter $4 \quad$ Fatty acid profile and affective dysregulation in irritable bowel syndrome

Chapter 5 Acute tryptophan depletion affects brain-gut responses in irritable bowel syndrome patients and controls

Chapter $6 \quad$ Acute tryptophan depletion alters cortical activation following visceral stimuli

Chapter $7 \quad$ The effects of an acute serotonergic challenge on brain-gut responses in irritable bowel syndrome patients and controls

Chapter 8 Rectal compliance and neurohormonal-immune signalling

143 in diarrhoea-predominant irritable bowel syndrome

Chapter $9 \quad$ General discussion

Summary

Samenvatting

Nawoord

Curriculum vitae 


\section{Abbreviations}

\begin{tabular}{|c|c|}
\hline AAAD & aromatic L-amino acid decarboxylase \\
\hline AA & arachidonic acid \\
\hline $\mathrm{ACC}$ & anterior cingulate cortex \\
\hline ACTH & adrenocorticotropic hormone \\
\hline ALA & a-linolenic acid \\
\hline ANS & autonomic nervous system \\
\hline ATD & acute tryptophan depletion \\
\hline BBB & blood brain barrier \\
\hline BMI & body mass index \\
\hline BOLD & blood oxygen level dependent \\
\hline BPRS & brief psychiatric rating scale \\
\hline CRH & corticotropin-releasing hormone \\
\hline CSF & cerebrospinal fluid \\
\hline CNS & central nervous system \\
\hline $\mathrm{DHA}$ & docosahexaenoic acid \\
\hline DPA & docosapentaenoic acid \\
\hline$d-\mid B S$ & diarrhoea-predominant irritable bowel syndrome \\
\hline EC & enterochromaffin \\
\hline EEG & electroencephalography \\
\hline ENS & enteric nervous system \\
\hline EPA & eicosapentaenoic acid \\
\hline FA & fatty acids \\
\hline FAME & fatty acid methyl ester \\
\hline fMRI & functional magnetic resonance imaging \\
\hline FGIDs & functional gastrointestinal disoders \\
\hline GHQ & general health questionnaire \\
\hline $\mathrm{Gl}$ & gastrointestinal \\
\hline GIT & groninger intelligence test \\
\hline HADS & hospital anxiety and depression scale \\
\hline HAM-D17 & 17-item hamilton depression rating scale \\
\hline HDL-C & high-density lipoprotein cholesterol \\
\hline HDRS & hamilton depression rating scale \\
\hline $\mathrm{HF}$ & high frequency \\
\hline HPA & hypothalamic-pituitary-adrenal \\
\hline HPLC & high performance liquid chromatography \\
\hline HRV & heart rate variability \\
\hline IBD & inflammatory bowel disease \\
\hline 5-HIAA & 5-hydroxyindole acetic acid \\
\hline $5-\mathrm{HT}$ & 5-hydroxytryptamine, serotonin \\
\hline 5-HTP & 5-hydroxytryptophan \\
\hline
\end{tabular}


IBS

IBSQOL

IL

I.V.

LA

LDL-C

LF

LNAAs

MAO

MEG

MINI

MMD

MSNA

MUFAs

Oba

PET

PCR

PI-IBS

POMS

PPP

PUFAs

RMBF

ROI

S1

SAFAs

SSRI

SCID

SCL

SERT

TCA

TNF- $\alpha$

TPH

VAS

Zung irritable bowel syndrome

irritable bowel syndrome quality of life questionnaire

interleukin

intravenous

linoleic acid

low-density lipoprotein cholesterol

low frequency

large neutral amino acids

monoamine oxidase

magnetoencephalography

mini international neuropsychiatric interview

minimum meaningful difference

muscle sympathetic nerve activity

monounsaturated fatty acids

osbond acid

positron emission tomography

polymerase chain reaction

postinfectious irritable bowel syndrome

profile of mood states

platelet poor plasma

polyunsaturated fatty acids

rectal mucosal blood flow

region of interest

primary sensory cortex

saturated fatty acids

selective serotonin reuptake inhibitor

structured clinical interview for DSM-IV

symptom checklist

serotonin reuptake transporter

tricyclic antidepressant

tumour necrosis factor-alpha

tryptophan hydroxylase

visual analogue scale

zung depression rating scale 


\section{Chapter 1}

General introduction 


\section{Irritable bowel syndrome a functional gastrointestinal disorder}

Functional gastrointestinal disorders (FGIDs) are common and often chronic disabling conditions accounting for a substantial percentage of primary care as well as about $40-60 \%$ of all referrals to gastroenterology clinics ${ }^{1}$. Moreover, these FGIDs are associated with significant work absenteeism, impaired health-related quality of life and high health-care utilization costs ${ }^{2}$. Within the concept of FGIDs irritable bowel syndrome (IBS), functional heartburn and functional dyspepsia are particularly common in the general population, with an overlapping symptom pattern and a tendency for transition between disorders over time. FGIDs classification is based on symptom-based criteria defined by an international group of experts during a consensus conference held in Rome in 1999 (Rome II criteria). For the history of the Rome diagnostic criteria see Table 1.1. Recently, the Rome III criteria, an update of the Rome II criteria, were released in the summer of $2006^{3,4}$.

IBS is described as a bowel disorder characterized by chronic or recurrent lower gastrointestinal (GI) tract symptoms that are conventionally not explained by structural abnormalities, infection, or metabolic changes. The Rome II criteria for IBS (as applied in this thesis) require abdominal discomfort or pain for at least 12 weeks or more, which need not to be consecutive, in the preceding 12 months. Also at least two out of three features (1) relieve with defecation; and/or (2) onset associated with a change in frequency of stool; and/or (3) onset associated with a change in form of stool are needed. IBS can be subdivided into constipation, diarrhoea, and the alternating constipation and diarrhoea type of IBS, respectively. Additional criteria for diarrhoeapredominant IBS (d-IBS) comprise more than three bowel movements a day or loose/watery stools or urgency ${ }^{5}$. Symptoms following ROME III criteria are now recommended to originate 6 months before diagnosis and be currently active i.e. at least three days per month in the last three months $s^{5,6}$. The age of onset of IBS varies, but the incidence appears to increase during adolescence and peaks in the third and fourth decade of life. About 10 to $20 \%$ of the Western population has symptoms consistent with a diagnosis of IBS with a female predominance of $2: 1^{5}$. Over the years IBS has been labelled by many terms such as spastic colon, mucus colitis and neurogenic colitis, often reflecting the state of pathophysiological understanding at the time $e^{7,8}$. 
$14 \mid$ Chapter 1

Table 1.1 History of the Rome Diagnostic Criteria (After Thompson) ${ }^{9}$.

The Manning Criteria for IBS (1978)
The Kruis Criteria for IBS (1984)
The Rome Guidelines for IBS (1989) (Rome-2 IBS Criteria)
The Rome Classification System for FGIDs (1990) (Rome-1)
The Rome I Criteria for IBS (1992) (14 $^{14}$ and the FGIDs (1994)
The Rome II Criteria for IBS $(1999)^{5}$ and the FGIDs (1999)
The Rome III Criteria (2006)
A historical perspective: disease, enteric nervous
system and serotonin

\begin{abstract}
Disease
The concept of body and mind being inseparable is not new. Ancient Greeks viewed illness from a "holistic" (Greek-holos) perspective. It reflects that in order to understand medical disease, one must take into account the whole person rather than just the affected disease part. A major paradigm shift occurred in Europe in 1637 through the influence of Rene Descartes, who proposed the separation of mind from the body (dualism), which replaced the holistic approach for at least 300 years. With Pasteur's discovery of microorganisms, and other discoveries in the $17^{\text {th }}$ and $18^{\text {th }}$ century relating to disease pathology, Western medicine moved further into the direction of the biomedical model of illness, characterized by reductionism and dualism ${ }^{16,17}$. However, as disease entities do not tend to restrict themselves to specific organs or organ systems and people with specific structural (organic) diagnoses show significant variation in their symptom presentation and clinical behaviour, medical science is slowly turning back to a more modernized holistic or biopsychosocial approach (initially described by George Engel in 1977) to medicine ${ }^{16,18}$. This model organizes systems in order of complexity and comprehensiveness, starting at the level of atoms and moving through cells, organs, individuals to family- and finally society level. Each system affects and is affected by other systems.
\end{abstract}

\title{
Enteric nervous system
}

Auerbach and Meissner discovered that the gut contains extremely large neural plexuses (later called enteric nervous system (ENS)). In the $19^{\text {th }}$ century Bayliss and Starling demonstrated that intestinal motility can be regulated by the ENS without input from the central nervous system (CNS) and they called this the law of the intestine. In 1917 Trendelenburg confirmed these findings by in vitro studying the guinea pig bowel and the law of the intestine became 
known as the peristaltic reflex. The independence of the ENS was incorporated by Langley into his description of the autonomic nervous system (ANS) including three divisions: the sympathetic (thoracolumbar), the parasympathetic (craniosacral), and the enteric (none). Because of the discrepancy in number of neurons between vagal $\left(10^{3}\right)$ and enteric neurons $\left(10^{8}\right)$, Langley suggested that most enteric neurons are not directly innervated by the CNS. After Langley the "classical" view of the ENS considered the neurons in the bowel wall to be "simple" parasympathetic relays between the CNS and its enteric effectors. Although the ENS by itself is able to control intestinal motility, the concept of bidirectional signalling between the CNS and ENS is only recently gaining interest $^{20}$.

\section{Serotonin}

In 1937, Erspamer isolated a compound from a subset of epithelial cells called the enterochromaffin $(E C)$ cells that would cause smooth muscle contractions. This substance was named "enteramine". In the same period Rapport et al., in search of a humoral agent responsible for hypertension, isolated and characterised an identical substance from serum that caused vasoconstriction and named it serotonin ${ }^{21}$. Later serotonin was biochemically determined to be 5-hydroxytryptamine (5-HT). The first isolation of serotonin from neural tissue in the brain occurred in 1953, and over the following decade the general pattern and distribution of serotonin-containing neurons throughout the entire CNS was discovered ${ }^{22}$.

\section{Current concept: enteric nervous system, brain-gut axis, serotonin}

\section{Enteric nervous system}

Gut function is modulated by both extrinsic and intrinsic pathways. The intrinsic innervation is provided by neurons of the myenteric and the submucosal plexuses, and the extrinsic innervation is provided by the spinal "sympathetic" and vagal-sacral "parasympathetic" nerves (Figure 1.1). Visceral signals are transmitted via ascending (e.g., spinothalamic and spinoreticular) pathways to the midbrain, thalamus, and cortex. The somatosensory cortex receives somatotopic information about the location and intensity of pain, whereas the limbic system (anterior cingulate cortex, insula, medial thalamus) is involved with the affective and motivational processing of pain. The latter system has the potential to modulate the pain experience, via activation of descending 
regulatory pathways (e.g., the endorphin-mediated analgesic system) to the dorsal horn and to up-regulate or down-regulate incoming visceral signals ${ }^{23}$.

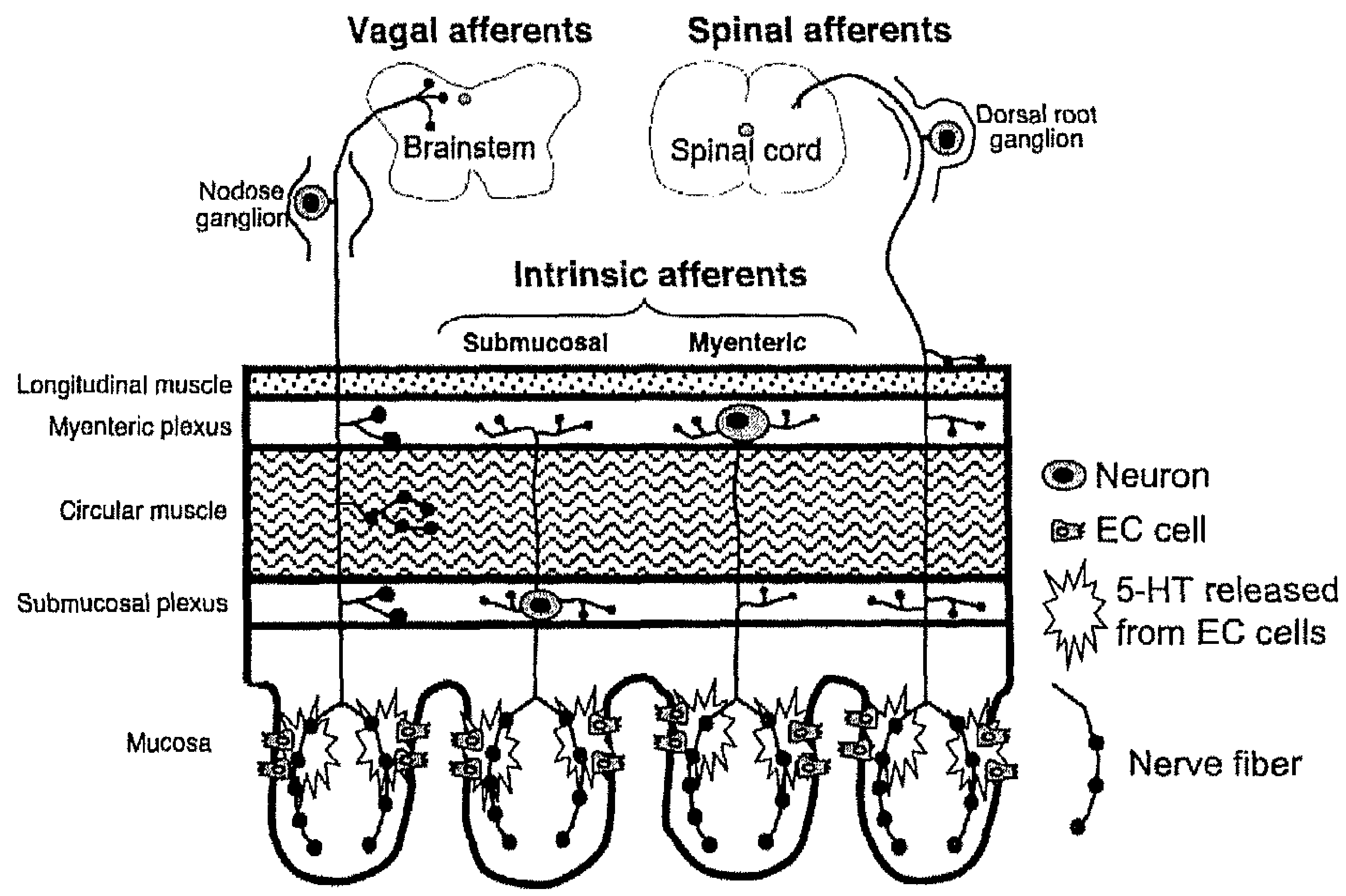

Figure 1.1 Schematic diagram depicting the branch patterns of intrinsic and extrinsic primary afferent neurons that innervate the intestinal mucosa. Intrinsic primary afferent nerve fibres originate from neurons with cell bodies located in the myenteric and submucosal plexuses. These neurons are involved in local activities such as motility, secretion and vasodilatation within the intestine. Extrinsic primary afferent nerves arise from neurons with cell bodies located in the nodose ganglia (vagal afferents) and dorsal root ganglia (spinal afferents). Extrinsic afferents transmit signals related to digestive reflexes, satiety, pain and discomfort from the gut to the central nervous system. As all these types of primary afferent neurons extend processes into the lamina propria of the intestines, they can be activated by serotonin (5-HT) released from enterochromaffin (EC) cells that are located in the crypt epithelium (after Mawe et al. $)^{38}$.

Irritable bowel syndrome; a disorder of brain-gut function

IBS research over the past half century shows a changing focus from psychological and stress-related research via emphasis on motility disturbances, to autonomic nervous system imbalance and visceral hypersensitivity ${ }^{24-27}$. FGIDs were initially attributed to motor dysfunction. By the late 1980s it had become apparent that the cause of most FGIDs could not be explained solely by motility disturbances. A series of contemporary studies suggested that patients with FGIDs might have a sensory dysfunction, which 
causes them to perceive physiological stimuli to the GI tract as GI symptoms ${ }^{28}$. The term visceral hypersensitivity indicates that a normally non-painful stimulus is experienced as painful (allodynia) or that the threshold for the perception of a painful stimulus is decreased (hyperalgesia). There is general agreement that IBS patients are more sensitive to distension of the sigmoid colon, ileum, and rectum than controls ${ }^{29}$. Given the high prevalence of sensory abnormalities in IBS, and the correlation of sensitivity with symptoms, altered visceral perception is considered to be a biological hallmark of $\mathrm{IBS}^{2}$. In addition to GI symptomatology, a high co-occurrence of IBS with affective dysregulation, mainly depression and anxiety, has been reported in gastroenterological settings. In addition, psychosocial stressors play a prominent role in symptom generation ${ }^{30-33}$. The high rates of affective dysregulation in IBS patients may be a specific and integral part of IBS, rather than a non-specific comorbidity related to chronic intestinal disease ${ }^{34-36}$. Visceral perception is known to be modulated at the cortical level and may therefore be influenced by cognitive mechanisms. The biopsychosocial approach of IBS attempts to offer an integrated model that reflects the complexity of IBS (Figure 1.2). In recent years, research has focused on the dysregulation of brain-gut interactions as the pathophysiological basis for IBS ${ }^{37}$. Summarizing, the pathophysiology of IBS remains incompletely understood, although research has shed light on a variety of putative pathophysiological mechanisms that provides a more integrated understanding of IBS as a brain-gut disorder.

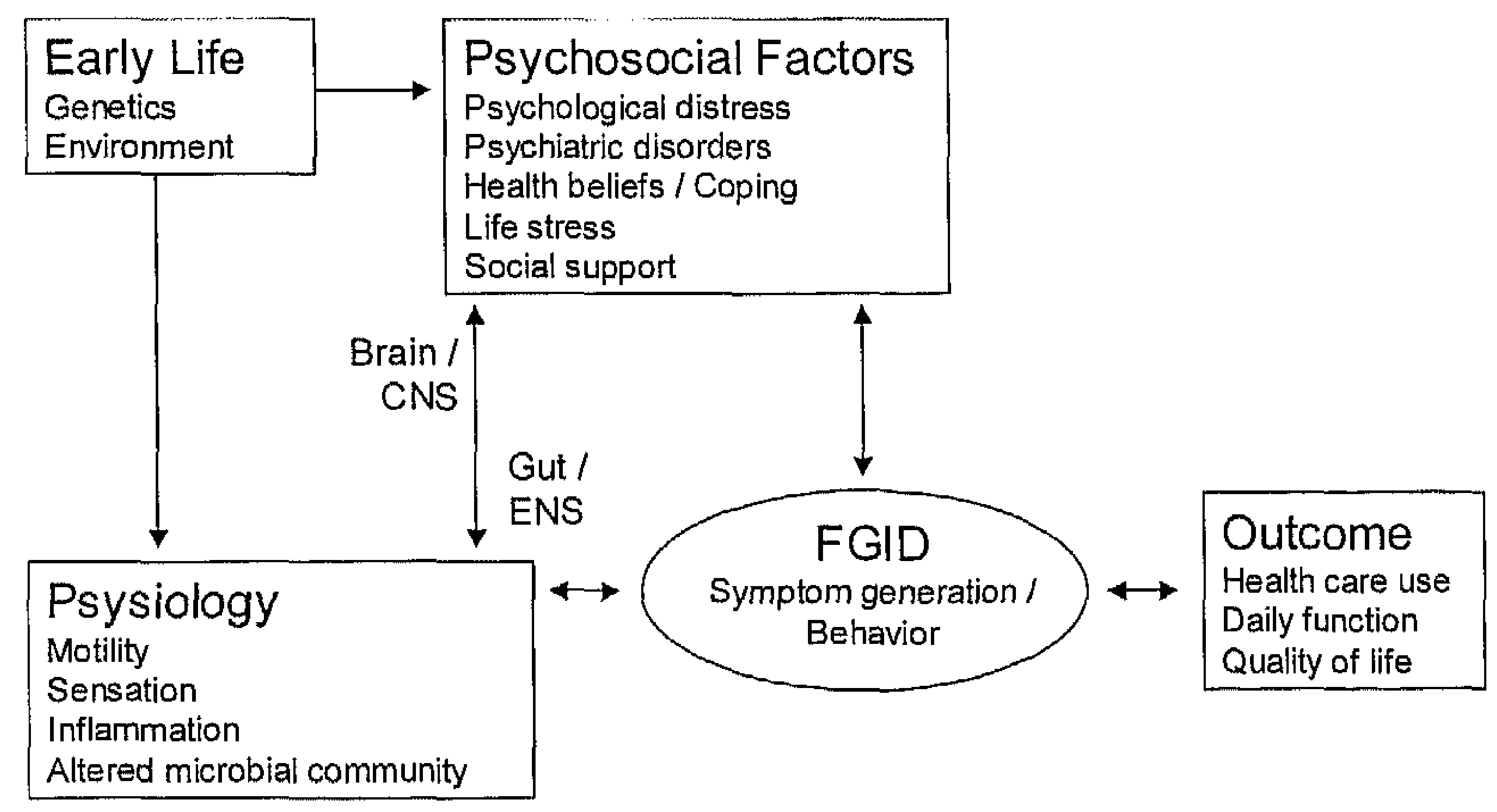

Figure 1.2 This biopsychosocial concept of the pathogenesis and clinical expression of functional gastrointestinal disorders (FGIDs) shows the relationship between early life, psychosocial and physiological factors, FGID symptoms, and clinical outcome (according to Ringel et al. ${ }^{19}$. CNS, central nervous system; ENS, enteric nervous system. 


\section{Serotonin}

Serotonin is often regarded as a neurotransmitter exclusive to the CNS, because of its roles in CNS functions including depression and anxiety. However, the major source of bioavailable 5-HT in the human body is located in the bowel, primarily in a subset of epithelial cells called enterochromaffin cells $^{38}$. Serotonergic dysregulation at CNS level is involved in the aetiology of many neuropsychiatric disorders including affective disorders such as depression and anxiety disorders ${ }^{39}$. Functional deficiencies in 5-HT are considered to be involved in the pathophysiology of depressive syndromes, and restoring the normal function of 5-HT-associated signalling pathways has been the target of antidepressant drugs ${ }^{40}$. Antidepressant therapy is recently recognized to be effective in the treatment of FGIDs ${ }^{36,41}$. Serotonin is currently regarded one of the key denominators involved in the brain-gut interaction. 


\section{General aim and outline of the thesis}

This thesis attempts to further elucidate the involvement of serotonin in braingut interactions and signalling with relevance to the pathophysiology of (diarrhoea-predominant) irritable bowel syndrome by addressing the following aspects:

- To determine (i) shared pathophysiological mechanisms in IBS and affective disorders and the role of serotonin by reviewing the co-occurrence of affective dysregulation in d-IBS and the influence of serotonergic treatment and (ii) to offer the framework for a new research paradigm in order to evaluate serotonergic modulation and assessment of integrated brain-gut function.

- To investigate effects of acute serotonergic modulation (increased and decreased serotonergic activity) on the brain-gut interaction by simultaneously investigating visceral sensory and motor function, cognition, mood, serotonergic parameters, brain activation and neuroendocrine responses.

- To study mediators of brain-gut signalling including (i) intermediary lipid metabolism (fatty acids and cholesterol) in association with affective symptomatology, and (ii) neurohormonal and immune signalling in relation with rectal motor physiology. 


\section{Outline of this thesis}

Chapter 2 is a comprehensive review regarding serotonergic modulation and integrated assessment of brain-gut function

Chapter 3 is a structured review of studies regarding presence of baseline $\mathrm{GI}$ and psychiatric symptomatology in IBS and the subsequent influence of serotonergic treatment on these $\mathrm{Gl}$ and psychiatric symptoms. In Chapter 4 an explorative study is presented on the fatty acid profile of plasma phospholipids and cholesterol in association with affective dysregulation in d-IBS and healthy controls. Following a randomised, double-blind crossover design, the effects of lowered serotonergic activity on visceral perception, cognition and mood in $\mathrm{d}$-IBS patients and controls are described in Chapter 5 using the acute tryptophan depletion (ATD) method. In Chapter 6 this method to lower 5-HT activity is used to study brain responses to visceral stimulation using functional magnetic resonance imaging (fMRI) in healthy females. Following a randomised, double-blind crossover design the effects of an acutely increased serotonergic activity by citalopram administration (a selective serotonin reuptake inhibitor) on visceral perception, cognition, mood and neuroendocrine response in d-IBS patients and controls are reported in Chapter 7 . The role of the intermediary metabolism within the concept of brain-gut signalling is evaluated in Chapter 8 by studying neurohormonal-immune mechanisms in relation to rectal motor physiology in $\mathrm{d}-\mathrm{JBS}$ and healthy controls.

In Chapter 9 the results of our studies are interpreted and discussed by addressing i) to what extend our results support the general concept of serotonergic involvement in brain-gut signalling with regard to IBS pathophysiology, ii) to what extend the results are applicable and constitute valid foundations for IBS research, clinical practice and therapy and iii) which recommendations can be made regarding future IBS research. 


\section{References}

1. Budavari $\mathrm{Al}$, Olden $\mathrm{KW}$. Psychosocial aspects of functional gastrointestinal disorders. Gastroenterol Clin North Am 2003;32:477-506.

2. Mertz H. Review article: visceral hypersensitivity. Aliment Pharmacol Ther 2003;17:623-633.

3. Drossman DA, Heaton K, Irvine EJ, Muller-Lissner S. Functional bowel disorders and functional abdominal pain. In: Drossman DA, Corazziari E, Talley N, Thompson WG, Whitehead WE, editors. The functional gastrointestinal disorders. Diagnosis, pathophysiology and treatment: A multinational consensus. McLean, VA: Degnon Associates; 2000:351-398.

4. Drossman DA. The functional gastrointestinal disorders and the Rome III process. Gastroenterology 2006;130:1377-1390.

5. Thompson WG, Longstreth GF, Drossman DA, Heaton KW, Irvine EJ, Muller-Lissner SA. Functional bowel disorders and functional abdominal pain. Gut 1999;45 Suppl 2:1143-1147.

6. Longstreth GF, Thompson WG, Chey WD, Houghton LA, Mearin F, Spiller RC. Functional bowel disorders. Gastroenterology 2006;130:1480-1491.

7. Bockus HI, Bank J, Wilkinson SA. Neurogenic mucous colitis. American Journal of Medical Science 1928;39:433-445.

8. Jacobson E. Spastic esophagus and mucous colitis: etiology and treatment by progressive relaxation. Archives of Internal Medicine 1927;39:433-445.

9. Thompson WG. The road to Rome. Gastroenterology 2006;130:1552-1556.

10. Manning AP, Thompson WG, Heaton KW, Morris AF. Towards positive diagnosis of the irritable bowel. Br Med J 1978;2:653-654.

11. Kruis W, Thieme $C$, Weinzierl M, Schussler $P$, Holl J, Paulus W. A diagnostic score for the irritable bowel syndrome. Its value in the exclusion of organic disease. Gastroenterology 1984;87:1-7.

12. Thompson WG, Dotevall G, Drossman DA, Heaton KW, Kruis W. Irritable bowel syndrome: guidelines for the diagnosis. Gastroenterol Int 1989;2:92-95.

13. Drossman DA, Funch-Jensen $P$, Janssens J, Talley $N J$, Thompson WG, Whitehead WE. Identification of subgroups of functional bowel disorders. Gastroenterol Int 1990;3:159-172.

14. Thompson WG, Creed FH, Drossman DA, Heaton KW, Mazzacca G. Functional bowel disorders and functional abdominal pain. Gastroenterol Int 1992;5:75-91.

15. Drossman DA, Richter JE, Talley NJ, Corazziari E, Thompson WG, Whitehead WE. Fucntional gastrointestinal disorders. Boston: Little, Brown; 1994.

16. Engel GL. The need for a new medical model: a challenge for biomedicine. Science 1977 196:129-136

17. Reading A. Illness and disease. Med Clin North Am 1977;61:703-710.

18. Halpert A, Drossman D. Biopsychosocial issues in irritable bowel syndrome. J Clin Gastroenterol 2005;39:665-669.

19. Ringel Y, Sperber A, Drossman D. Irritable bowel syndrome. Annu Rev Med 2001;52:319-38.

20. Gershon MD. Serotonin and its implication for the management of irritable bowel syndrome. Rev Gastroenterol Disord 2003;3 Suppl 2:S25-34.

21. Page IH. The discovery of serotonin. Perspect Biol Med 1976;20:1-8.

22. Saxena PR. Historical aspects of 5-hydroxytryptamine, discovery and receptor classification. New York: Raven Press; 1992.

23. Azpiroz F. Intestinal perception: mechanisms and assessment. Br J Nutr 2005;93 Suppl 1: S7-12.

24. Welgan $P$, Meshkinpour $H$, Hoehler $F$. The effect of stress on colon motor and electrical activity in irritable bowel syndrome. Psychosom Med 1985;47:139-149.

25. Kellow JE, Langeluddecke PM, Eckersley GM, Jones MP, Tennant CC. Effects of acute psychologic stress on small-intestinal motility in health and the irritable bowel syndrome. Scand J Gastroenterol 1992;27:53-58.

26. Aggarwal A, Cutts TF, Abell TL, Cardoso S, Familoni B, Bremer J, Karas J. Predominant symptoms in irritable bowel syndrome correlate with specific autonomic nervous system abnormalities. Gastroenterology 1994;106:945-950. 
27. Bueno L, Fioramonti J, Delvaux M, Frexinos J. Mediators and pharmacology of viscera sensitivity: from basic to clinical investigations. Gastroenterology 1997;112:1714-1743.

28. Azpiroz F. Hypersensitivity in functional gastrointestinal disorders. Gut 2002;51 Suppl 1: i25-28.

29. Delvaux M. Role of visceral sensitivity in the pathophysiology of irritable bowel syndrome. Gut 2002;51 Suppl 1:i67-71

30. Noyes R, Jr., Cook B, Garvey M, Summers R. Reduction of gastrointestinal symptoms following treatment for panic disorder. Psychosomatics 1990;31:75-79.

31. Walker EA, Katon WJ, Jemelka RP, Roy-Bryne PP. Comorbidity of gastrointestinal complaints, depression, and anxiety in the Epidemiologic Catchment Area (ECA) Study. Am $J$ Med 1992;92:26S-30S.

32. Zaubler TS, Katon W. Panic disorder and medical comorbidity: a review of the medical and psychiatric literature. Bull Menninger Clin 1996;60:A12-38.

33. Lydiard RB. Anxiety and the irritable bowel syndrome: psychiatric, medical, or both? J Clin Psychiatry 1997;58:51-58; discussion 59-61.

34. Walker EA, Roy-Byrne PP, Katon WJ, Li L, Amos D, Jiranek G. Psychiatric iliness and irritable bowel syndrome: a comparison with inflammatory bowel disease. Am J Psychiatry 1990;147:1656-1661.

35. Fock KM, Chew CN, Tay LK, Peh LH, Chan S, Pang EP. Psychiatric illness, personality traits and the irritable bowel syndrome. Ann Acad Med Singapore 2001;30:611-614.

36. Kilkens TO, Honig A, Rozendaal N, Van Nieuwenhoven MA, Brummer RJ. Serotonergic modulators in the treatment of irritable bowel syndrome - influence on psychiatric and gastrointestinal symptoms. Aliment Pharmacol Ther 2003;17:43-51.

37. Wood JD, Alpers DH, Andrews PL. Fundamentals of neurogastroenterology. Gut 1999;45 Suppl 2:I6-II16.

38. Mawe GM, Coates MD, Moses PL. Review article: intestinal serotonin signaling in irritable bowel syndrome. Aliment Pharmacol Ther 2006;23:1067-1076.

39. Blier P, Abbott FV. Putative mechanisms of action of antidepressant drugs in affective and anxiety disorders and pain. J Psychiatry Neurosci 2001;26:37-43.

40. Elhwuegi AS. Central monoamines and their role in major depression. Prog Neuropsychopharmacol Biol Psychiatry 2004;28:435-451.

41. Jackson JL, O'Malley PG, Tomkins G, Balden E, Santoro J, Kroenke K. Treatment of functional gastrointestinal disorders with antidepressant medications: a meta-analysis. Am J Med 2000;108:65-72. 


\section{Chapter 2}

Review article: serotonergic modulation and assessment of integrated brain-gut function in irritable bowel syndrome

TO Kilkens, RJ Brummer

Submitted 


\section{Summary}

Although irritable bowel syndrome (IBS) is generally considered as a disorder of the brain-gut axis it remains a complex and not fully understood syndrome. Serotonin is one of the key denominators involved in brain-gut axis signalling and function.

This review proposes a framework for investigating brain-gut function and the potential role for serotonin in brain-gut signalling. In this report we specifically focus on the assessment of integrated brain-gut function and serotonergic modulation paradigms including the acute tryptophan depletion method and the citalopram (SSRI) challenge test in healthy subjects in general and in IBS patients in particular. Several available biomarkers of brain-gut function are presented including biomarkers at "end-organ" level i.e. at brain and gut level and mediators of brain-gut signalling including autonomic nervous system, neuroendocrine-immune, intermediary and serotonergic systems, respectively.

In order to achieve breakthroughs in pharmacological and nutritional interventions in IBS the multicomponent nature of IBS pathophysiology should be taken into consideration. Serotonergic modulation of the brain-gut axis by pharmacological or nutritional means and subsequent assessment of integrated brain-gut function offers the possibility to gain further insight into the role of serotonergic regulation and brain-gut function in functional gastrointestinal disorders.

\section{Contents}

* Introduction

* Brain-gut axis and serotonin in irritable bowel syndrome

-The brain-gut concept

Gut

Brain

Autonomic nervous system (ANS)

Neuroendocrine-immune function

Intermediary metabolism

-Serotonin

-Involvement of serotonin in the brain-gut axis

Gut

Brain

Autonomic nervous system (ANS)

Neuroendocrine-immune function

Intermediary metabolism

* Biomarkers of integrated brain-gut function

- Parameters of end-organ function

Gastroenterological and psychiatric diagnosis and symptomatology

Gut

Brain

Mood and cognition

-Mediators of brain-gut signalling

Autonomic nervous system

Neuroendocrine-immune function

Intermediary metabolism

Serotonergic function

* Serotonergic modulation techniques

-Acutely decreasing serotonergic activity by acute tryptophan depletion

Mechanism and procedure

Effects on brain-gut function

Irritable bowel syndrome

-Increasing serotonergic activity by citalopram administration

Mechanism and procedure

Effects on brain-gut function

Irritable bowel syndrome

* Conclusions

* References 
Serotonergic modulation and assessment of brain-gut function $\mid 25$

\section{Introduction}

The irritable bowel syndrome (IBS) is a common functional gastrointestinal disorder (FGID) characterized by chronic or recurrent lower gastrointestinal (GI) tract symptoms that are not conventionally explained by structural abnormalities, infection, or metabolic changes. About 10 to $15 \%$ of the Western population has symptoms consistent with a diagnosis of IBS, and they account for as many as $40 \%$ of all new referrals to the gastroenterologist ${ }^{1}$. A better understanding of the pathophysiological mechanisms underlying IBS may help to develop more effective pharmacological or even nutritional interventions for this disorder. Although extensive research has indicated that altered intestinal motor physiology and visceral hypersensitivity contribute to the symptom generation in IBS, the pathophysiology of IBS remains not fully understood. A multicomponent conceptual model of IBS has been postulated, integrating physiologic, affective, cognitive and behavioural factors ${ }^{2}$. According to the brain-gut axis model, the brain and the gut are highly integrated and interact through bi-directional communication pathways connecting the enteric nervous system (ENS), the central nervous system (CNS), the autonomic nervous system (ANS), neuroendocrine centres, and the immune system (Figure 2.1) (2,3 $^{2}$. IBS is now generally considered as a disorder primarily involving the brain-gut axis $^{3}$. Serotonin (5-HT) is one of the key denominators involved in the brain-gut interaction ${ }^{4}$. A recent review summarized the evidence for altered 5-HT signalling in IBS at the level of the gut ${ }^{5}$. The authors concluded that as a universal aetiology for IBS is not likely to exist, it is unlikely that one given defect or alteration is responsible for the various changes associated with this syndrome $e^{5}$. Although the concept of the brain-gut axis is commonly accepted, assessment of integrated brain-gut function is still in its infancy. Consequently, serotonergic modulation of the brain-gut axis by pharmacological or nutritional means and subsequent assessment of integrated brain-gut function offers the possibility to gain further insight into the role of serotonergic regulation and brain-gut function. In this report we specifically focus on the assessment of integrated brain-gut function and serotonergic modulation paradigms in healthy subjects in general and in IBS patients in particular. Firstly, we describe the brain-gut axis concept and the involvement of $5-\mathrm{HT}$. Secondly, we focus on available biomarkers of integrated brain-gut function at the level of the gut, the brain and mediators of brain-gut axis signalling, respectively. Finally, we describe serotonergic modulation techniques with an emphasis on the acute tryptophan depletion (ATD) method and the citalopram (selective serotonin reuptake inhibitor, SSRI) challenge test and applications of these techniques within brain-gut research. We conclude with describing the opportunities and future perspectives of serotonergic modulation and the assessment of integrated brain-gut function. 
$26 \mid$ Chapter 2

Biomarkers

Visceral perception Cognition

Mood

CNS processing/activity

5-HT metabolism/signalling

Sympatho- vagal balance Immune/inflammatory parameters

Neuroendocrine hormones Lipid profile

5-HT metabolism/signalling

Motor function

Low-grade inflammation

Extrinsic nerve

Autonomic activity

5-HT metabolism/signalling

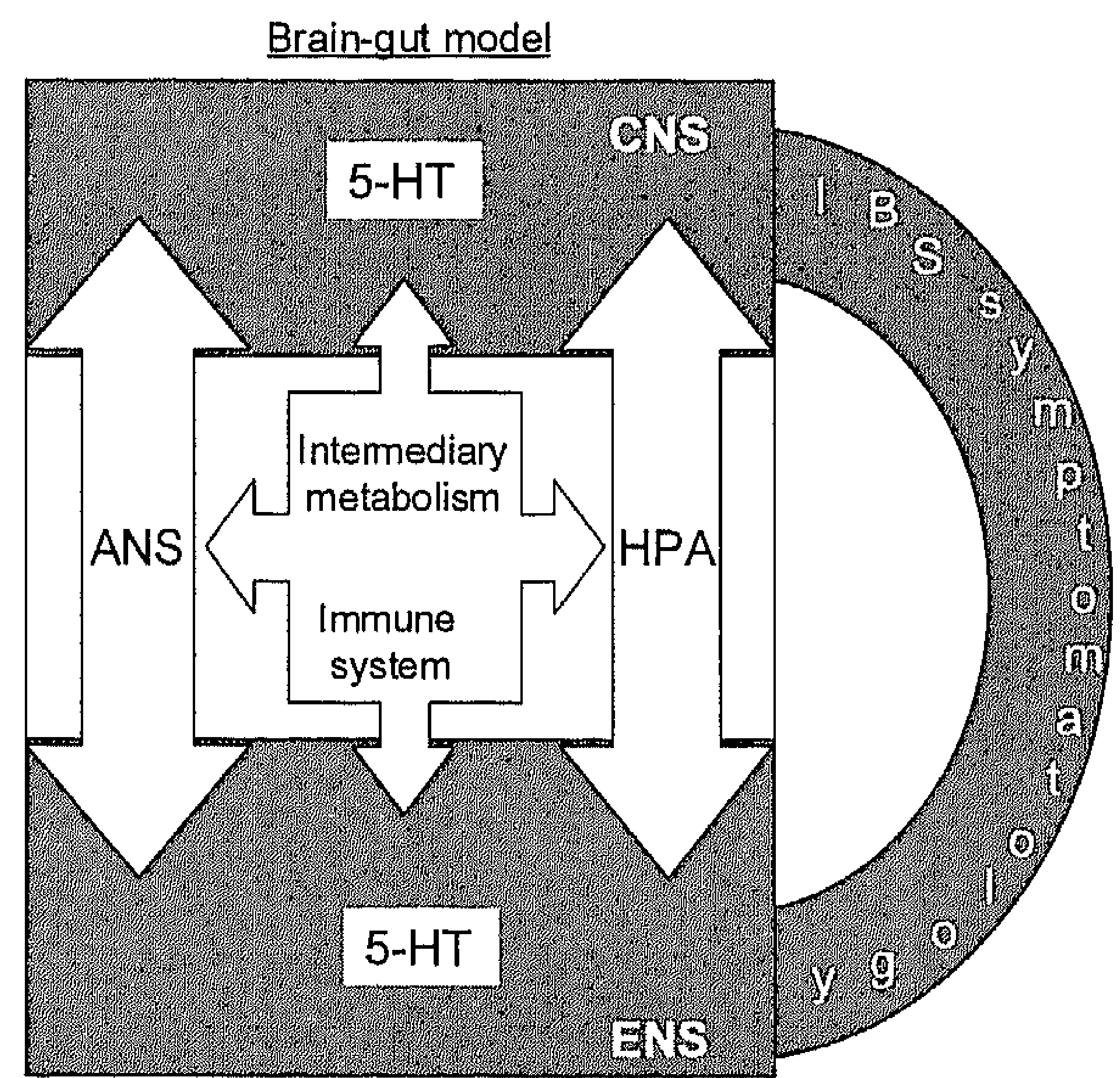

Figure 2.1 Schematic representation of an integrated brain-gut axis model displaying a "blackbox" of communication pathways connecting several domains comprising the central nervous system (CNS), the enteric nervous system (ENS), neuroendocrine centres (hypothalamic-pituitary-adrenal (HPA) axis), the autonomic nervous system (ANS), the immune system and intermediary metabolism. Gastrointestinal symptom generation can be traced back to the level of either the brain or the gut. Irritable bowel syndrome (IBS) research which integratively determines the various biomarkers of these domains along the brain-gut axis may facilitate the translation from brain-gut axis dysregulation to IBS symptomatology. As depicted, serotonin (5-HT) plays a major role in the modulation of the brain-gut connection.

\section{Brain-gut axis and serotonin in irritable bowel syndrome}

\section{The brain-gut concept}

Gut

Control and modulation of $\mathrm{Gl}$ function occurs both locally in the gut wall by intrinsic neural pathways called the enteric nervous system (ENS), and extrinsically by vagal and spinal nerves connecting CNS and ENS. Although the ENS is capable of functioning independently of CNS control, the extrinsic nervous system conveys information bi-directionally via afferent and efferent pathways between the ENS and the CNS ${ }^{6}$.

Exaggerated intestinal motor responses have been shown to occur after stress and meal ingestion in IBS, and also aberrations in intestinal wall compliance 
are considered to be a consistent feature of IBS ${ }^{7,8}$. Given the high prevalence of sensory abnormalities in IBS, and the correlation of visceral sensitivity with symptoms, altered rectal perception has been considered to be a biological hallmark of $\mathrm{IBS}^{9}$. Enhanced visceral perception (hypersensitivity) and motor responses (compliance, contraction, accommodation) may result both from peripheral (e.g. inflammation, infection) or central (e.g. attention, anticipation, and mood) sensitisation mechanisms.

\section{Brain}

In IBS, a high proportion (40 to $94 \%$ ) of co-occurrence with psychiatric axis I diagnoses has been described including depression, anxiety and somatization disorders ${ }^{10}$. Although psychiatric comorbidity is most prevalent in tertiary care settings, the comorbidity rates are higher than in general medical patients or patients with organic Gl disorder such as inflammatory bowel disease (IBD). It is now well acknowledged that psychiatric comorbidity, and psychosocial stressors play a prominent role in IBS symptom generation ${ }^{11}$.

Depressive disorders are known to be characterised by a substantial impairment in cognitive function (e.g. memory impairment) and affective changes (lowered mood). "Mood congruent" attentional bias toward negative emotional material and an attentional bias away from positive emotional cues are present in patients with major depressive disorder ${ }^{12}$. Pathophysiological changes in IBS occur at the level of the brain which have a high similarity with those characteristic of affective disorders. It can be hypothesized that CNS mechanisms result in increased attention and hypervigilance to $\mathrm{Gl}$ sensations in IBS and influence $G$ f function and sensation ${ }^{13}$. The modulatory role of psychological factors in visceral perception is illustrated by the fact that a stress signal decreases pain thresholds, and that distraction and relaxation increase pain thresholds, respectively ${ }^{14}$. Conversely, abnormal $\mathrm{Gl}$ function may influence the CNS. This is supported by the fact that visceral signals tend to activate brain regions such as the anterior cingulate cortex (ACC) associated with unpleasant affect and autonomic responses ${ }^{15}$. This region is of particular interest in both affective disorder and IBS ${ }^{16,17}$. Abnormal CNS processing of visceral stimuli in IBS has been postulated ${ }^{15}$. Summarizing, psychiatric disorders may not only represent epiphenomena to IBS but these disorders may share pathophysiological mechanisms.

\section{Autonomic nervous system (ANS)}

The ANS is anatomically and functionally integrated with the brain-gut axis ${ }^{18}$. Increased sympathetic and decreased parasympathetic activity are the most frequently noted ANS-associated features of IBS $^{19}$. Affective and cognitive factors are capable of altering autonomic balance and major depressive and 
$28 \mid$ Chapter 2

anxiety disorders have also been associated with autonomic imbalance ${ }^{20}$. Some studies have reported that anxiety and depression are related to ANS function in IBS ${ }^{21}$. As regions involved in the central processing of $\mathrm{Gl}$ sensation overlap with brain regions involved in emotional and autonomic regulation, this may explain how these systems interact in IBS ${ }^{22}$.

\section{Neuroendocrine-immune function}

Abnormal neuroendocrine-immune interactions may contribute to altered $\mathrm{GI}$ physiology and hypersensitivity in at least a subset of patients with $\mathrm{IBS}^{23,24}$. Activation of brain corticotropin-releasing hormone (CRH) receptor signalling pathways has been implicated in stress-related endocrine responses, the development of anxiety-like behaviour, and in stress-related stimulation of colonic function and hypersensitivity ${ }^{25,26}$. In accordance with the brain-gut model recent advances in psychoneuro-immunology have shown that the CNS and immune system are intimately linked. There is evidence for alterations in the activity of the hypothalamic-pituitary-adrenal (HPA) axis and the immune system (proinflammatory response) underlying the pathophysiology of major depressive disorder ${ }^{27}$. Interaction of the bidirectional communication between the immune system and the neuroendocrine system takes place through both neuroendocrine outflow via the HPA axis, and through direct neural influences through sympathetic, parasympathetic (cholinergic) and peptidergic/sensory innervation of peripheral tissues including lymphoid organs, blood vessels and serotonergic pathways ${ }^{28}$.

\section{Intermediary metabolism}

Reports in recent years have shown that lipid metabolism interacts with CNS and immune function ${ }^{29,30}$. Polyunsaturated fatty acids (PUFAs) are precursors of eicosanoids (prostaglandins, thromboxanes, prostacyclines and leukotrienes) $)^{31}$. Eicosanoids derived from $n-3$ PUFAs have weaker inflammatory properties than those derived from n-6 PUFAs. Although the precise mechanisms explaining the effects of PUFAs remain to be clarified, modifications of physical properties of the neuronal membrane, and effects on gene expression and/or post-transcriptional modification may occur ${ }^{32}$. Specific changes in the composition of PUFAs, cholesterol and the immune profile have been observed in patients with affective disorders and inflammatory bowel disease (IBD) ${ }^{29,33}$. Dietary supplementation of $n-3$ PUFAs has been shown to improve affective dysregulation in major depressive disorder and to be beneficial in the treatment of many inflammatory conditions ${ }^{34,35}$. Whether these findings suggest involvement of the PUFAs profile as a metabolic mediator in the brain-gut interaction remains to be elucidated. More knowledge of PUFAs 
metabolism in IBS patients may have both pathophysiological and therapeutic implications $^{36}$.

Summarizing, the brain-gut axis consists of bidirectional communication pathways connecting the cognitive and emotional brain centres, with neuroendocrine centres, the ENS, the ANS and the immune system. Hence, alterations at any level between the brain and the gut may influence IBS symptoms by autonomic, cognitive-affective, neuroendocrine-immune and intermediate mechanisms (Figure 2.1). Serotonin (5-HT) is a pivotal neurotransmitter involved in the regulation of these domains.

\section{Serotonin}

Serotonin is a biogenic amine that functions as a neurotransmitter and paracrine signalling molecule. It is involved in a number of diverse physiologic functions including mood, appetite, sleep, memory and learning, vasoconstriction, haemostasis, behaviour and immune responses. More than $80 \%$ of total body $5-\mathrm{HT}$ is located in the GI tract; with $90 \%$ in enterochromaffin (EC) cells and $10 \%$ in enteric neurons, respectively. The remainder of $5-\mathrm{HT}$ is located in dense core storage vesicles in blood platelets or in the CNS, mainly in the raphe nuclei.

Serotonin is synthesized in a two-step reaction from the essential amino acid tryptophan $^{37}$. The availability of tryptophan influences serotonin synthesis (Figure 2.2). The majority of 5-HT in blood is derived from release by EC cells in the gut wall and is functionally inactive and stored in blood platelets or metabolised by the liver and kidney. Hence, 'free' concentrations of 5-HT in plasma are very low. This supports the evidence that $5-\mathrm{HT}$ in blood exists in two distinct pools, a rapid turnover pool in plasma which is sensitive to acute change and a slow turnover pool in platelets, respectively ${ }^{38}$. Gastrointestinal 5HT may be released via a number of mechanisms including chemical stimuli, vagal stimulation and application of pressure to the mucosa ${ }^{39} .5-\mathrm{HT}$ receptors are classified into seven $5-\mathrm{HT}$ receptor families $\left(5-\mathrm{HT}_{1}-5-\mathrm{HT} \mathrm{T}_{7}\right)$ and multiple subtypes. A 5-HT transporter called serotonin reuptake transporter (SERT) mediates $5-H T$ reuptake. SERT in the brain (serotonergic neurons) is similar to that in the gut (epithelial cells and enteric neurons) ${ }^{40}$. Serotonin can be metabolised, as a result of the activity of the enzyme monoamine oxidase (MAO) to 5-hydroxyindole acetic acid (5-HIAA) ${ }^{41}$. A model of a synapse depicting $5-\mathrm{HT}$ metabolism and the proteins of interest is presented in Figure 2.2. 
$30 \mid$ Chapter 2

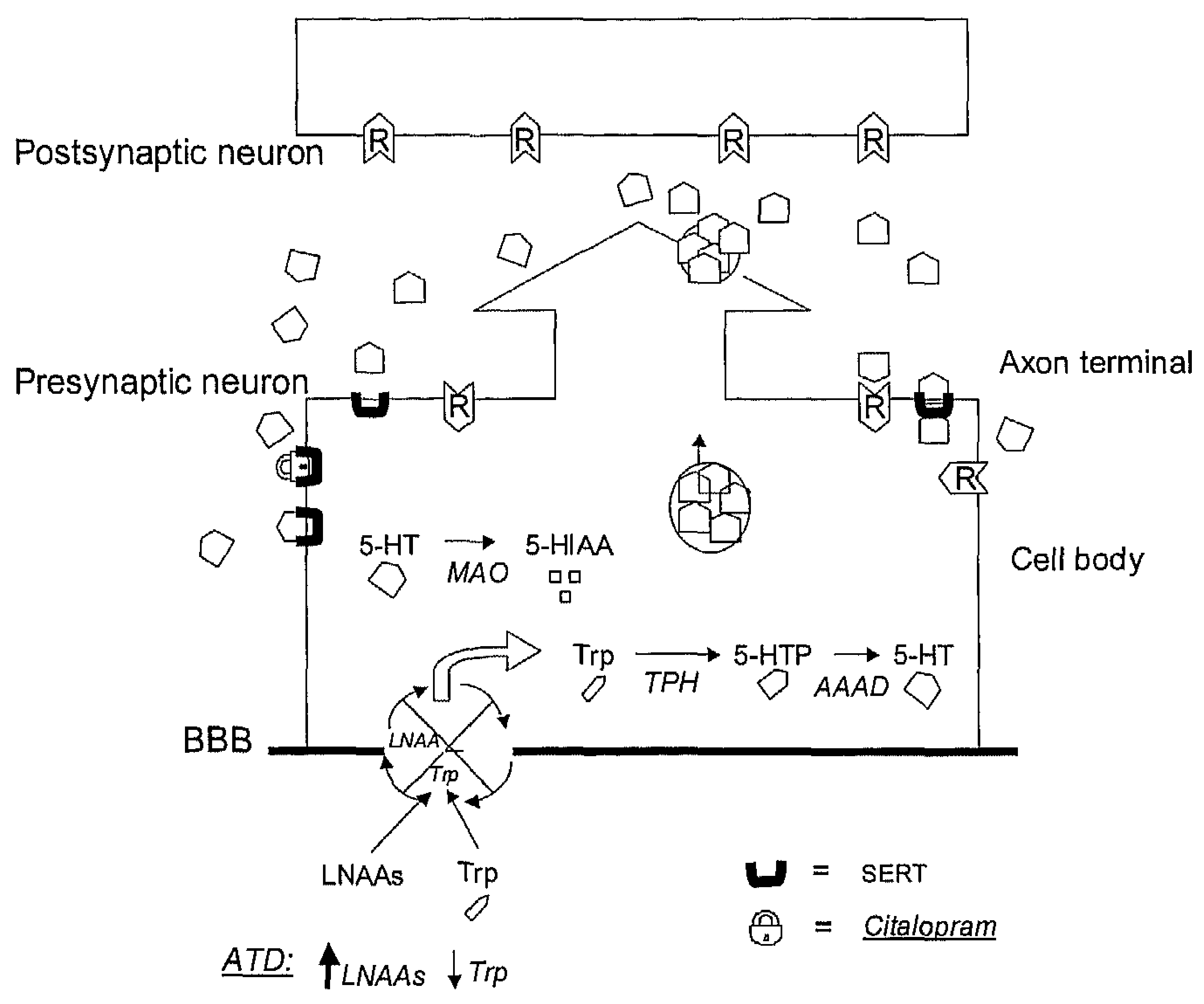

Figure 2.2 Simplified serotonin synapse. Serotonin $(5-\mathrm{HT})$ is shown from synthesis to synaptic release and then binding to pre- and postsynaptic receptors ( $R$ ). Brain tryptophan (Trp) uptake depends on the serum levels of tryptophan and several other large neutral amino acids (LNAAs), which compete for transport at the blood brain barrier (BBB) (revolving door model) ${ }^{42}$. Serotonin is synthesized from tryptophan by two enzymatic steps. Firstly, hydroxylation of tryptophan by the enzyme tryptophan hydroxylase (TPH) (the activity of which is rate limiting) produces 5-hydroxytryptophan (5-HTP). The second enzymatic step is decarboxylation of 5-HTP by the enzyme aromatic L-amino acid decarboxylase (AAAD) producing 5-HT. 5-HT is inactivated primarily by reuptake by the serotonin reuptake transporter (SERT). Serotonin can be very rapidly metabolised by monoamine oxidase (MAO) to 5 -hydroxyindole acetic acid (5-HIAA).

ATD: An acute decrease of $5-H T$ activity can be achieved by means of the Acute Tryptophan Depletion (ATD) method which lowers the tryptophan concentration and increases the concentration of large neutral amino acids (LNAAs).

Citalopram: An acute increase of 5-HT activity can be achieved by administration of the selective serotonin reuptake inhibitor (SSRI) citalopram. SSRIs block the reuptake of 5-HT into the presynaptic neuron by the SERT and consequently increase 5-HT levels in the synapse. 
Serotonergic modulation and assessment of brain-gut function $\mid 31$

Involvement of serotonin in the brain-gut axis

Gut

5-HT initiates $\mathrm{Gl}$ function responses as diverse as nausea, vomiting, vascular tone, perception, intestinal secretion, and peristalsis ${ }^{43}$. Recent evidence indicates that various alterations in serotonergic content, release, and reuptake at the level of the gut and systemically in platelet free plasma may be involved in IBS pathophysiology $y^{5,44}$. We recently observed that lowering $5-\mathrm{HT}$ activity enhances visceral perception and slows gastric emptying ${ }^{45,46}$.

Brain

At the CNS level, 5-HT is involved in mood regulation and cognition ${ }^{47}$. Serotonergic dysregulation at CNS level plays a role in the aetiology of many neuropsychiatric disorders including affective disorders such as depression and anxiety disorders ${ }^{48}$. Functional deficiencies in $5-H T$ have been implicated in the pathophysiology of depressive syndromes, and restoring the normal function of 5-HT-associated signalling pathways has been the target of antidepressants ${ }^{49}$. Antidepressant therapy has also been applied in the treatment of FGIDs ${ }^{50,51}$.

\section{Autonomic nervous system}

Regarding ANS function, central serotonergic activity plays a significant role in the reflex control of heart rate and blood pressure ${ }^{52}$. Antidepressant treatment has been associated with changes in vagal modulation and a potential role for SSRIs has been suggested in patients with autonomic disturbances ${ }^{53,54}$.

\section{Neuroendocrine-immune function}

There is an increasing volume of evidence to link 5-HT with HPA axis regulation ${ }^{55}$. Neuroendocrine release is controlled by hypothalamic neurons, many of which are innervated by serotonergic nerve terminals. Stimulation of central serotonergic neurotransmission as well as pro-inflammatory cytokines have the ability to trigger the activation of the HPA axis by provoking the release of hormones such as $\mathrm{CRH}$ and vasopressin, adrenocorticotropic hormone (ACTH), cortisol and prolactin ${ }^{56-58}$. In addition, both the HPA axis and serotonergic mechanisms are involved in the immune response by modulating the production of cytokines ${ }^{28,59}$.

\section{Intermediary metabolism}

The role of lipid metabolism, especially that of PUFAs and cholesterol in the pathophysiology of affective disorders has not only been related to attenuated immunological responses but also to alterations in CNS serotonergic 
$32 \mid$ Chapter 2

neurotransmitter systems, membrane-bound receptor function, and enzymatic activity caused by disruption of neuronal membrane composition and structure $^{60}$.

Summarizing, 5-HT signalling plays a major role in the modulation of the braingut connections and hence altered 5-HT signalling may affect both intestinal and extraintestinal symptom generation in IBS (Figure 2.1).

\section{Biomarkers of integrated brain-gut function}

As outlined above, brain-gut interactions are bidirectional. Therefore, it is possible that $\mathrm{Gl}$ symptomatology is due to enhanced visceral signalling (bottom-up), altered CNS input to the ENS (top-down), aberrant CNS perception of normal visceral afferent signalling or a combination of these possibilities. Also the communication pathways between CNS and ENS may be structurally damaged (disease), or more likely, functionally altered (disorder), possibly by alterations in ANS, neuroendocrine-immune, intermediary or serotonergic functioning. Thus, biomarkers of communication pathways enable monitoring of the intrinsic function of the route itself as well as brain-gut signalling during both basal as well as challenged (e.g. stressors, meal ingestion) conditions.

It is difficult to establish the primacy of any of the components of the brain-gut axis in IBS symptom generation, as one may affect the other. In order to achieve breakthroughs in IBS research the multi-component nature of IBS pathophysiology should be taken into consideration. Therefore, it is essential not to examine one isolated aspect of pathophysiology. One should try to assess integrated brain-gut function by simultaneous measurement of biomarkers along the brain-gut axis. Several available biomarkers of brain-gut function are presented below. Firstly, biomarkers at "end-organ" level, i.e. at brain and gut level are presented. Secondly, mediators of brain-gut signalling are discussed including ANS, neuroendocrine-immune, intermediary and serotonergic systems, respectively.

\section{Parameters of end-organ function}

\section{Gastroenterological and psychiatric diagnosis and symptomatology}

Current guidelines recommend assessment of "psychological distress or prior mental health" in trials of psychological intervention or psychoactive drugs and these psychological factors are regarded as potential confounders in nonpsychological treatments ${ }^{61}$. In our opinion, future studies should not only 
Serotonergic modution and assessment of brimgut function $\mid 33$

apply the ROME criteria for IBS but should also screen for potential psychiatric diagnosis, to improve our understanding of the pathology in IBS (e.g. by using the Structured Clinical Interview for the DSM-IV (SCID) or Mini International Neuropsychiatric Interview (MINI) $)^{62,63}$. In addition the momentary presence and severity of $\mathrm{Gl}$ symptoms and psychiatric symptomatology can be determined (the latter by using e.g. the Hospital Anxiety and Depression Scale (HADS), Hamilton Depression Rating Scale (HDRS), Beck Depression Inventory (BDI-II), Symptom Checklist-90-Revised (SCL-90-R) ${ }^{64-67}$. These scales are suitable for tracking (short-term) changes in severity over time, not only in a research setting but also for clinical follow-up.

\section{Gut}

Assessment of visceral sensitivity requires probing stimuli of different intensities applied within the gut (e.g. mechanical, chemical, electrical, thermal) and appropriate means of evaluating the elicited responses. For an overview regarding the principles of investigating sensation and motility testing the reader is referred to recent reviews ${ }^{68,69}$. An often applied stimulus comprises a pressure-controlled gut distension. The barostat device has been designed to evaluate the sensitivity to a distension stimulus (visceral perception) and the pressure-volume relations (e.g. tone, compliance) of hollow organs. Using this isobaric approach, a volume expansion reflects a relaxation, and a volume reduction a contraction. The reproducibility, sensitivity, specificity and predicative value of rectal sensory testing in IBS have been carefully characterized ${ }^{70,71}$. The intensity and the quality of individuals' subjective visceral perception can be measured by means of standardized rating scales.

\section{Brain}

In order to gain more pathophysiological insight and for more objective and direct assessment of specific CNS network activation in response to visceral stimuli advances have been made by functional brain imaging and electrophysiological techniques. This is usually performed in conjunction with rectal distension in order to provide an adequate visceral afferent signal that elicits a detectable response in the CNS. Positron emission tomography (PET) and functional magnetic resonance imaging (fMRI) are the most commonly used techniques. PET assesses blood flow in the brain or regional cerebral metabolism after i.v. injection of a radiolabeled compound. fMRI is used to measure increases in local oxygenated haemoglobin level (blood oxygenation level-dependent (BOLD) responses) as an indirect measure of increased blood flow, a marker of focal brain activity. Other techniques include electroencephalography (EEG) and magnetoencephalography (MEG). Discussion of these techniques and their application in FGID is beyond the 
scope of this article and is covered in recent reviews ${ }^{17,72,73}$. It remains difficult to determine whether changes in visceral perception arise from peripheral afferent dysfunction or from (non-specific) central changes ${ }^{74-76}$. Even brain imaging techniques do not have sufficient temporal resolution to differentiate between activity directly related to the stimulus and the activity that is produced endogenously representing higher cognitive functions ${ }^{75}$. In IBS approaches to overcome these problems are EEG in combination with independent component analysis and functional neuroimaging of subliminal (below perception) visceral stimulation ${ }^{75,77}$. The former has the potential to reveal the dynamic activation in network processing of visceral stimulation, the latter has been proposed to evaluate visceral afferent/sensory neural circuitry irrespective of stimulus/perception-related cognitive processes ${ }^{78,79}$. The concept of subliminal stimulation in order to overcome response bias seems to be attractive. However, response bias has been demonstrated even for subliminal stimuli (also called nonconscious, preattentive or masked stimuli) in patients with depression and anxiety disorder and in children with recurrent abdominal pain $^{80-82}$.

\section{Mood and cognition}

The assessment of mood, cognition and attentional interference / response bias can be performed to gain insight into potential CNS mechanisms influencing GI perception. Subjective mood status can be assessed with visual analogue scales with e.g. profile of mood states (POMS) ${ }^{83}$. However, response bias may offer a more objective and sensitive biomarker. This psychological response bias can be demonstrated by affective word recall and $/$ or concomitant registration of event-related brain potentials of word processing ${ }^{46,84}$.

\section{Mediators of brain-gut signalling}

\section{Autonomic nervous system}

ANS function in relation to the cardiovascular system is generally considered to be representative for general autonomic function and hence applied in GI research. Frequently used basal parameters include: heart rate, blood pressure, baroreflex sensitivity and beat-to-beat heart rate variability (HRV). Other tests include those of sudomotor function. The most direct measurement of sympathetic activity is by directly recording bursts of efferent muscle sympathetic nerve activity (MSNA) ${ }^{85,86}$. Finally, a more specific assessment of autonomic function of the gut is reflected by rectal mucosal blood flow (RMBF) determined by laser doppler flowmetry. Although it is unclear whether RMBF is representative for the entire gut this method seems to correlate with 
Serotonergic modulation and assessment of brain-gut function $\mid 35$

cardiovagal tone ${ }^{87}$. Autonomic nervous system status can be determined with prolonged recordings of HRV and responsiveness of the ANS can be assessed by applying various standardized autonomic challenge manoeuvres including the Valsalva manoeuvre, deep (metronomic) breathing, and orthostatic challenge. It would be preferable to include meal ingestion, visceral distension or application of physical (cold water hand immersion) or psychological stress (dichotomous listening) as relevant stimuli in ANS function measurements in $\mathrm{IBS}^{88}$.

\section{Neuroendocrine-immune function}

Neuroendocrine status is reflected by basal (unstimulated) HPA activity, which can be assessed by measuring circulating levels of neuroendocrine hormones. Diurnal patterns of e.g. cortisol profiles may be of additional value as HPA axis activity displays a circadian rhythm. The HPA axis can be acutely stimulated by administration of $\mathrm{CRH}$ analogues or by acute serotonergic probes/ challenges ${ }^{57,89}$. Regarding immune activity, distribution of circulating immune cells can be assessed by measuring absolute leukocytes and lymphocyte subset counts and quantitative analysis of lymphocyte subsets ${ }^{90}$. Free plasma cytokine or receptor levels rather than stimulated whole blood or peripheral blood mononuclear cells may reflect the actual biological activity of the cytokines $^{91}$. Immune system responsiveness can be assessed after nutrient load or other psychological or physiological stressors ${ }^{90}$.

Although conventional histology of rectal biopsy specimens in IBS patients may appear normal, the presence of low-grade immune activation can be demonstrated by quantification of immune cell (e.g. macrophages, lymphocytes, mast cells) and EC cell populations using immunohistology and cytokine mRNA expression using reverse transcriptase-polymerase chain reaction $(P C R)^{92}$. A novel technique which absorbs and then extracts inflammatory mediators from cellulose patches compressed against the rectal mucosa, called the "mucosal patch technique" may also be useful to study subclinical intestinal inflammation ${ }^{93}$. The relation between cytokine gene polymorphisms and the association between systemic and mucosal inflammatory markers has recently gained interest ${ }^{94,95}$. Interestingly, the ability to exogenously attenuate (e.g. by fish oil) the inflammatory response may depend on the inherent cytokine production and by gene polymorphisms for cytokines $^{96}$.

\section{Intermediary metabolism}

Instead of determining lipid intake in IBS patients it is possible in this research domain to determine a lipid profile, a biomarker of intermediary metabolism related to inflammation, immune pathways, affective regulation and 
36 Chanter

serotonergic function. Cholesterol can routinely be measured in serum. Profiles of FA can be measured as components of plasma phospholipids or cholesterol esters (past few days), erythrocyte membranes (reflecting last month), and adipose tissue (long-term dietary intake) from various sites ${ }^{97}$. The interpretation and relevance of fatty acid profile as a modulatory intermediary pathway is based on the relative percentage an individual fatty acid contributes to the total of all FA (weight percentages (wt\%) of total FA), instead of the absolute amount of each $F A^{98}$.

\section{Serotonergic function}

Concentration of tryptophan, the precursor of 5-HT, can be determined in plasma or serum ${ }^{99}$. In addition, the ratio of tryptophan/ $/ 2$ LNAAs (large neutral amino acids such as tyrosine, valine, leucine, isoleucine, and phenylalanine) can be determined. This ratio is considered to be a more accurate estimate of tryptophan transport into the brain ${ }^{100}$. Concentrations of whole blood 5-HT are approximately equivalent to that of platelet $5-\mathrm{HT}$, and are indicative of more long-term changes in 5-HT metabolism. Determination of 5-HT in platelet poor plasma is the preferred assessment of acute changes and small differences in $5-\mathrm{HT}$ metabolism ${ }^{101}$. Peripheral SERT can be measured by expressing, binding and reuptake properties of the SERT molecule. Quantitative PCR can be used to quantify SERT mRNA expression in e.g. immune cells. An estimate of the density (maximal binding capacity $(B \max )$ ) and binding affinity (dissociation constant $(K d)$ ) of this transporter can be quantified by e.g. [3H]paroxetine or $[3 \mathrm{H}]$ citalopram binding and reuptake properties of SERT by $[3 \mathrm{H}] 5-\mathrm{HT}$ uptake in e.g. platelets ${ }^{102}$. The level of $5-H T$ breakdown can be measured as platelet MAO activity ${ }^{103}$. Cerebrospinal fluid 5-HIAA levels, indicative of central serotonergic activity, significantly correlate to platelet MAO activity ${ }^{104}$. The major metabolite of serotonin, 5-HIAA, can be determined in blood and urine. In blood, 5-HT turnover (5-HIAA:5-HT) is regarded as a surrogate marker of peripheral 5-HT metabolism ${ }^{105}$.

Gut specific 5-HT measurements (using biopsy specimens) comprise mucosal content and release of 5-HT, mucosal 5-HT turnover (5-HIAA:5-HT), quantitative measurement of serotonin-containing EC cell numbers, SERT measurement by immunoreactivity intensity and measurement of mRNA encoding SERT and TPH1 using PCR ${ }^{106}$.

Since direct measurement of synaptic serotonergic activity in the CNS is not possible in humans, the neuroendocrine response to serotonergic probes or cerebrospinal fluid (CSF) 5-HT metabolite concentrations have been used in a psychiatric setting as an indirect parameter of central serotonergic function ${ }^{107}$. As will be mentioned later in this review, aberrations of the neuroendocrine response to serotonergic stimulation in patients with affective disorders have been shown ${ }^{108}$ 
Many studies have used blood platelets in the peripheral circulation as a peripheral model of $5-\mathrm{HT}$ neurons ${ }^{109}$. However, such peripheral models may fail to differentiate between localized brain regions and specific pathophysiological processes.

in vivo imaging of CNS SERT with selective radiotracers (e.g. [123I]ADAM, [123l]h-CIT) can be performed using PET and this provides an important tool to study the availability and activity of SERTs in living human brain ${ }^{110}$. Another potentially valuable recent technique that needs further validation comprises a combination of pharmacological challenges and $\mathrm{fMRI}$; the so-called direct pharmacoMRI (pMRI). This technique has the potential to detect and localise functional $5-\mathrm{HT}$ effects on brain function by acute i.v. administration of citalopram as a pharmacological challenge ${ }^{111}$.

Candidate genes involved in 5-HT function may encode pivotal enzymes in 5HT metabolism (TPH and MAO), SERT and 5-HT receptors. Genes encoding various components of the $5-\mathrm{HT}$ system are being studied as risk factors as well as efficacy parameters of drugs affecting the $5-\mathrm{HT}$ system in psychiatric disorders like depression and anxiety ${ }^{112}$. The most extensively studied functional polymorphism is the one coding for the SERT molecule. SERT polymorphism has been associated with altered neuroendocrine and neuromodulatory responses following citalopram administration and response to SSRI treatment in mood disorders ${ }^{113,114}$.

Data on SERT polymorphism in IBS are equivocal ${ }^{115-118}$. It needs to be elucidated to what extend functional responses of the serotonin system may contribute to IBS symptom generation and whether these responses represent a neurobiological substrate for the differential response to antidepressant treatment ${ }^{119}$.

\section{Serotonergic modulation techniques}

Acutely decreasing serotonergic activity by acute tryptophan depletion

\section{Mechanism and procedure}

An acute decrease of central 5-HT activity can be achieved by means of the acute tryptophan depletion (ATD) method. ATD selectively lowers 5-HT synthesis, between four and seven hours after oral administration of an amino acid mixture devoid of the 5-HT precursor tryptophan. The amino acid drink contains 15 amino acids dissolved in $200 \mathrm{ml}$ tap water in the same proportion as in human milk, except that tryptophan, aspartic acid and glutamic acid are omitted (Table 2.1) ${ }^{120}$. A tryptophan-containing drink serves as a placebo. A 
$38 \mid$ Chapter 2

common ATD paradigm involves two test days about 1 week apart in a doubleblind, crossover design. The amino acid load stimulates protein synthesis, which requires tryptophan. Tryptophan is incorporated into proteins which leads to a $60-70 \%$ decrease of plasma tryptophan levels. Brain tryptophan uptake depends on the serum levels of tryptophan and other LNAAs, which compete for transport at the BBB (revolving door model)(Figure 2.2) ${ }^{42}$. In human subjects, substantial reductions of brain 5-HT synthesis and decreased levels of CSF 5-HIAA have been demonstrated after ATD ${ }^{121}$. Studies with PET in healthy subjects confirmed these data, by showing that ATD decreases brain 5HT synthesis by $90 \%$ or more ${ }^{122,123}$.

Until now, ATD has been used with regard to changes in CNS functioning and only plasma tryptophan/LNAAs ratios have been routinely assessed as a surrogate marker of central serotonergic depletion. However, we have provided evidence that ATD exerts its metabolic action also at the level of the gut as ATD significantly decreased plasma 5 -HIAA concentrations ${ }^{46}$.

Acute tryptophan depletion is a relatively inexpensive, non-invasive method of serotonergic modulation which is safe and acceptable to human investigation ${ }^{124}$. Reducing tryptophan by administration of long-term diets has been shown to reduce plasma tryptophan levels by only $15-20 \%$ and to have few behavioural effects ${ }^{125}$. Moreover, long periods of dieting seem both unethical and impractical.

Table 2.1 Composition of the amino acid drink. Amounts of each of the specific amino acid according to the proportions described by Young et al. ${ }^{120}$.

\begin{tabular}{lc}
\hline Amino Acid & Standard Weight (g) \\
\hline L-alanine & 4.1 \\
L-arginine & 3.7 \\
L-cysteine & 2.0 \\
Glycine & 2.4 \\
L-histidine & 2.4 \\
L-lysine & 6.7 \\
L-methionine & 2.3 \\
L-proline & 9.2 \\
L-serine & 5.2 \\
L-threonine & 4.7 \\
LNAAs: & \\
L-isoleucine & 6.0 \\
L-leucine & 10.1 \\
L-phenylalanine & 4.3 \\
L-tyrosine & 5.2 \\
L-valine & 6.7 \\
& \\
L-tryptophan & 3.0 \\
Total (minus L-tryptophan) & \\
Total (plus L-tryptophan) & 75 \\
\hline
\end{tabular}


Serotonergic modulation and assessment of brain.gut function $\mid 39$

\section{Effects on brain-gut function}

ATD has been used in a psychiatric setting to investigate the role of the central $5-\mathrm{HT}$ system in patients with affective disorders (depression and anxiety) and their first-degree relatives. A transient lowering of mood was the first reported behavioural effect of ATD in humans ${ }^{120}$. However, subsequent studies in euthymic healthy subjects without a family history of depression report no change of mood symptoms during ATD ${ }^{126}$. Women may be more prone to the effects of ATD compared to men ${ }^{127}$. This may be in line with the evidence that there may be a sex difference in 5-HT metabolism and the increased prevalence of depressive disorder and IBS in women compared to men ${ }^{123}$. An increased vulnerability to mood alteration during ATD has also been reported in euthymic subjects who have a family history of affective illness ${ }^{128}$. An SS polymorphism (i.e. two short alleles) of the promoter region of the SERT gene and a positive family history of depression are additional risk factors for the development of mood changes during ATD ${ }^{129}$. ATD induces transient symptoms in $50-60 \%$ of those with remitted depression treated with serotonergic agents ${ }^{130}$. ATD does not worsen the symptoms of currently depressed patients (potentially because of a floor effect) and induces no symptoms or only mild dysphoria in healthy volunteers ${ }^{120}$. This seems conflicting with a simple model of the pathogenesis of depression based on a low 5-HT activity. The concept of serotonergic vulnerability may describe more adequately the association between serotonin and depression and potentially between serotonin and IBS ${ }^{130}$.

Most studies reported that ATD had very little effect on ratings of anxiety in both healthy controls and patients with panic disorder. However, combining ATD with an anxiety-provoking challenge induces small increases in ratings of nervousness in healthy volunteers and in patients with untreated panic disorder it tends to result in an increased rate of panic attacks ${ }^{131}$.

The most consistent finding from studies in healthy volunteers and vulnerable subjects is an adverse effect of ATD on cognitive measures of delayed recall and recognition ${ }^{132}$. Studies have suggested that ATD produces changes in emotional processing and these result in a shift of affective memory bias towards negative or mood-congruent affective stimuli ${ }^{133}$.

One study concerning the effects of ATD on ANS function showed a decreased HRV in a specific group of remitted depressed patients ${ }^{134}$.

Studies regarding immune function and ATD suggest that ATD does not promote an inflammatory cytokine response in healthy volunteers ${ }^{135,136}$. ATD induced, however, a transient reduction in soluble interleukin (sIL-4) levels (anti-inflammatory cytokine) related to depression ratings in remitted patients with seasonal affective disorder ${ }^{137}$. 
$40 \mid$ Chapter 2

\section{Iritable bowel syndrome}

We provided the first evidence that ATD influences $G$ function and brain-gut interactions. ATD affects GI physiology by delaying gastric emptying in healthy females and ATD affects both visceral perception as well as cognition (affective memory bias) in d-IBS patients and healthy matched controls ${ }^{45,46}$. ATD may lead to a shift from primary visceral pain sensation, as represented by activation of the right insula and the inferior frontal operculum, towards a more affective or emotional experience of the visceral pain sensation, as represented by increased activation of the ACC, superior frontal cortex and right insula (see Chapter 6).

\section{Increasing serotonergic activity by citalopram administration}

\section{Mechanism and procedure}

The selective serotonin reuptake inhibitors (SSRIs) constitute a class of agents of which the principle mode of action is blocking the reuptake of 5-HT into the presynaptic neuron. Thus, these agents augment the extracellular concentration of serotonin. Citalopram is the SSRI with the highest selectivity for the SERT, with no known intrinsic activity at any 5-HT receptor subtype or at any other neurotransmitter system ${ }^{138}$. Citalopram is well-tolerated and effective in the treatment of major depression and panic disorder ${ }^{139}$. An acute increase of 5-HT activity can be achieved by either i.v. or oral administration of citalopram. Doses used (5-10-20-30 mg) appear to be safe and well tolerated $d^{108,140,141}$. The i.v. placebo procedure consists of $0.9 \%$ saline solution. The use of parenteral rather than oral citalopram administration reduces pharmacokinetic variability ${ }^{140}$. Although citalopram is known for its central effects on 5-HT function it may have also a peripheral influence on 5-HT metabolism ${ }^{111,142}$. We demonstrated increased platelet poor plasma 5-HT levels after $20 \mathrm{mg}$ i.v. administration of citalopram ${ }^{143}$.

It appears that SSRIs are effective against depression not only because of their acute effects on serotonergic transmission. Clinical improvement is associated with the long-term adaptive changes in monoamine neurotransmission arising from the chronic inhibition of serotonin reuptake. Converging preclinical evidence suggests that mechanisms underlying this response timeline involve a variety of adaptive neurochemical changes including aminergic reuptake inhibition and associated presynaptic autoregulatory desensitization, up- and downregulation of multiple postsynaptic receptor sites, and receptor-mediated second messenger and neurotrophic effects ${ }^{144}$. 


\section{Effects on brain-gut function}

Acute i.v. administration of citalopram significantly enhances memory performance and reduces the processing of negative relative to positive emotional material without notable subjective mood differences in healthy controls $^{145}$. A four-week treatment study with citalopram in patients with major depression showed that induced changes of the cortisol response pattern correlated with improvement of working memory ${ }^{146}$. Furthermore, significant reductions in both interpretive bias and anxiety levels in patients with a diagnosis of generalized anxiety disorder were reported ${ }^{147}$.

The effects of acute citalopram administration on ANS function are yet unclear. Studies suggest that SSRIs may have a sympatho-inhibitory effect ${ }^{148}$.

The neuroendocrine response induced by a citalopram challenge has been used in a psychiatric setting as an indirect measure of central serotonergic function ${ }^{107,141}$. Kapitany et al. showed significantly blunted prolactin secretion in depressed patients, whereas differences in cortisol secretion following serotonergic stimulation with citalopram did not reach significance ${ }^{108}$. Decreased availability of 5-HT reuptake in mid-brain and brain-stem regions in patients with depression could contribute to decreased functional responses to 5 -HT challenge identified by neuroendocrine tests ${ }^{149}$. Smith et al. demonstrated that SERT polymorphism is associated with an altered functional response of the serotonin system following i.v. citalopram in healthy volunteers ${ }^{150}$. Hence, variability in acute responses to $5-\mathrm{HT}$ challenge probes, determined by either neuroendocrine responses or functional imaging, can be used to measure variability in the function of the 5-HT system.

There are no systematic studies on the effects of acute citalopram administration on immune function or intermediary metabolism. However, treatment with different types of SSRIs demonstrated that IL-6 levels significantly decreased in patients with major depression as well as in controls $^{59}$. In patients with anxiety disorders effective treatment with a SSRI may decrease cholesterol levels ${ }^{151}$.

\section{Irritable bowe/ syndrome}

Gorard et al. were the first to assess the effects of acute oral fenfluramine (a $5-\mathrm{HT}$ releasing agent) in patients with IBS. They did not demonstrate significant differences in neuroendocrine responses between patients with IBS, inflammatory bowel disease patients or healthy controls, respectively ${ }^{152}$. We demonstrated that $20 \mathrm{mg}$ i.v. citalopram administration enhanced memory performance and induced an affective memory bias towards positive material without significantly influencing the subjective mood status or rectal perception in d-IBS and healthy controls. In addition, citalopram significantly increased plasma $5-\mathrm{HT}, \mathrm{ACTH}$ and cortisol levels compared with placebo in these 
42 Chapter 2

groups. Although citalopram-induced neuroendocrine responses were significantly negatively associated with the level of affective dysregulation, neuroendocrine responses did not differentially affect the IBS patient or control group ${ }^{143}$. Tack et al. demonstrated that in healthy subjects rather than IBS acute i.v. citalopram administration induced an increase in colonic phasic contractility and the occurrence of high-amplitude propagated contractions, increased colonic compliance and a suppression of the colonic tonic response to a meal ${ }^{153,154}$.

In the first placebo-controlled treatment trial using citalopram Tack et al. showed that citalopram significantly improved IBS symptoms including abdominal pain after six weeks treatment (three weeks $20 \mathrm{mg}$, three weeks $40 \mathrm{mg}$ ) in non-depressed IBS patients. Interestingly, this therapeutic effect was independent of effects on anxiety, depression and colonic sensorimotor function ${ }^{154}$. Regarding long-term SSRI treatment including paroxetine and fluoxetine in IBS some improvement in abdominal pain and overall well-being has been reported ${ }^{155-158}$.

\section{Conclusions}

Irritable bowel syndrome remains a complex and not fully understood syndrome. In order to achieve breakthroughs in IBS research the multicomponent nature of IBS pathophysiology should be taken into consideration. This review proposed a framework for investigating integrated brain-gut function and the potential role for serotonin $(5-\mathrm{HT})$ in brain-gut signalling.

In this review we focused mainly on acute serotonergic modulatory paradigms including the acute tryptophan depletion method and citalopram challenge test. It should be taken into consideration that an acute, temporary limited modulation of 5-HT levels may not be a valid model for chronic and more severe alterations in 5-HT metabolism or signalling. In addition, as there are multiple 5-HT receptors subtypes the question remains where the alterations in the signalling process occur. Hence, future studies which combine these paradigms with 5-HT receptor-specific agonists and antagonists such as $5-\mathrm{HT}_{3}$ antagonists and $5-\mathrm{HT}_{4}$ agonists could be of potential interest regarding receptor-specific function in brain-gut regulation ${ }^{159}$. Furthermore, relationships between systemic, gastrointestinal and CNS 5-HT metabolism and the influence of acute and chronic serotonergic modulation on each of these systems needs further elucidation. Pharmacological or nutritional modulation at one level consequently modifies efferent and afferent activity at other levels of the brain-gut axis. 
Currently, it remains difficult if not impossible to determine whether changes in brain-gut functioning arise from specific or even non-specific peripheral or central alterations or both. Investigating the principles of brain-gut axis dysregulation and attempts to assess "integrated" brain-gut function seems like solving the "chicken-and-egg problem". Brain imaging techniques such as combined analysis of EEG and $\mathrm{FMRI}$ data, EEG in combination with independent component analysis and magnetoencephalography (MEG) may yield insight in brain-gut processing and symptom generation ${ }^{76,160}$.

In order to assess integrated brain-gut function several available biomarkers of brain-gut function have been presented. Several confounding factors should be taken into consideration in order to make valid between-group comparisons of biomarkers. These include gender differences, menstrual cycle, age, (family) history of psychiatric disorder and multiple physiological (e.g. postprandial testing) and psychological factors (e.g. stress) ${ }^{161-165}$.

It is unlikely that all IBS patients turn out to have 5-HT-related symptom generation. The framework of integrated brain-gut assessment we presented is also suitable for other challenges apart from 5-HT modulation paradigms. Also noradrenergic pathways seem to play a role in the pathophysiology of both depression and $\mathrm{IBS}^{166,167}$. Hence, dual acting antidepressants such as serotonin-norepinephrine reuptake inhibitors (SNRIs) appear interesting regarding brain-gut modulation.

ATD could be regarded as a proof of principle that 5-HT activity can be modulated by nutritional means. However, increase of central serotonergic activity, rather than tryptophan depletion, is regarded advantageous with regard to brain-gut regulation and could result in improved mood, cognition and visceral sensitivity. Foods that elevate brain tryptophan levels are rich in carbohydrates rather than proteins. It is hypothesized that carbohydrateinduced insulin secretion reduces plasma levels of LNAAs, leading to enhanced $5-\mathrm{HT}$ brain content by reducing the competition for tryptophan uptake. On the other hand, high-protein foods are rich in amino acids that compete with tryptophan, thus reducing its entry into serotonergic neurons ${ }^{168}$. Evidence suggests that the use of tryptophan as a supplement or dietary source may be used as a therapeutic or preventive intervention in subjects with mild depression or those at risk, respectively, which may be of relevance for IBS $^{169,170}$.

In summary, assessing brain-gut function is clearly important in functional gastrointestinal disorders. Biomarkers of brain-gut function are undergoing further validation. In the present review, the role of serotonin and integrated 
44 Chapter 2

brain-gut function assessment has been presented. Various aspects concerning the assumed link between alterations in serotonergic signalling and brain-gut dysregulation via enteric nervous system, hypothalamic-pituitaryadrenal axis and neurohormonal-immune interactions in IBS have been discussed. A better understanding of these interacting domains will significantly improve our understanding of IBS symptom generation and potentially of other functional gastrointestinal disorders, and will hopefully lead to the development of novel pharmaceutical and nutritional interventions for this common and debilitating disorder. 


\section{References}

1. Drossman DA, Heaton $K$, Irvine EJ, Muller-Lissner S. Functional bowel disorders and functional abdominal pain. In: Drossman DA, Corazziari E, Talley N, Thompson WG, Whitehead WE, editors. The functional gastrointestinal disorders. Diagnosis, pathophysiology and treatment: A multinational consensus. McLean, VA: Degnon Associates; 2000:351-398.

2. Mayer EA. Emerging disease model for functional gastrointestinal disorders. Am J Med 1999; 107:12S-19S.

3. Jones MP, Dilley JB, Drossman D, Crowell MD. Brain-gut connections in functional $G$ disorders: anatomic and physiologic relationships. Neurogastroenterol Motil 2006;18:91-103.

4. Kim DY, Camilleri M. Serotonin: a mediator of the brain-gut connection. Am J Gastroenterol 2000;95:2698-2709.

5. Mawe GM, Coates MD, Moses PL. Review article: intestinal serotonin signaling in irritable bowel syndrome. Aliment Pharmacol Ther 2006;23:1067-1076.

6. Gershon MD. Nerves, reflexes, and the enteric nervous system: pathogenesis of the irritable bowel syndrome. J Clin Gastroenterol 2005;39:S184-193.

7. Zar S, Benson MJ, Kumar D. Rectal afferent hypersensitivity and compliance in irritable bowel syndrome: differences between diarrhoea-predominant and constipation-predominant subgroups. Eur J Gastroenterol Hepatol 2006;18:151-158.

8. Camilleri M, Ford MJ. Review article: colonic sensorimotor physiology in health, and its alteration in constipation and diarrhoeal disorders. Aliment Pharmacol Ther 1998;12:287-302.

9. Mertz H, Naliboff B, Munakata J, Niazi N, Mayer EA. Altered rectal perception is a biological marker of patients with irritable bowel syndrome. Gastroenterology 1995;109:40-52.

10. Whitehead WE, Palsson $O$, Jones KR. Systematic review of the comorbidity of irritable bowel syndrome with other disorders: what are the causes and implications? Gastroenterology 2002;122:1140-1156.

11. Lydiard RB. Anxiety and the irritable bowel syndrome: psychiatric, medical, or both? J Clin Psychiatry 1997;58:51-8; discussion 59-61.

12. Leppanen JM. Emotional information processing in mood disorders: a review of behavioral and neuroimaging findings. Curr Opin Psychiatry 2006;19:34-39.

13. Whitehead WE, Palsson OS. Is rectal pain sensitivity a biological marker for irritable bowel syndrome: psychological influences on pain perception. Gastroenterology 1998;115: 1263-1271.

14. Accarino AM, Azpiroz F, Malagelada JR. Attention and distraction: effects on gut perception. Gastroenterology 1997;113:415-422.

15. Derbyshire SW. Visceral afferent pathways and functional brain imaging. Scientific World Journal 2003;3:1065-1080.

16. Ebmeier KP, Donaghey C, Steele JD. Recent developments and current controversies in depression. Lancet 2006;367:153-167.

17. Derbyshire SW. A systematic review of neuroimaging data during visceral stimulation. Am J Gastroenterol 2003;98:12-20.

18. Tougas $G$. The autonomic nervous system in functional bowel disorders. Can J Gastroenterol 1999;13 Suppl A:15A-17A.

19. Tillisch K, Mayer EA, Labus JS, Stains J, Chang L, Naliboff BD. Sex specific alterations in autonomic function among patients with irritable bowel syndrome. Gut 2005;54:1396-1401.

20. Critchley HD. Neural mechanisms of autonomic, affective, and cognitive integration. J Comp Neurol 2005;493:154-166.

21. Jarrett ME, Burr RL, Cain KC, Hertig V, Weisman P, Heitkemper MM. Anxiety and depression are related to autonomic nervous system function in women with irritable bowel syndrome. Dig Dis Sci 2003;48:386-394.

22. Mertz $\mathrm{H}$. Role of the brain and sensory pathways in gastrointestinal sensory disorders in humans. Gut 2002;51 Suppl 1:i29-33.

23. Spiller R, Campbell E. Post-infectious irritable bowel syndrome. Curr Opin Gastroenterol 2006;22:13-17. 
24. Dinan TG, Quigley EM, Ahmed SM, Scully P, O'Brien $S$, O'Mahony L, O'Mahony $S$, Shanahan F, Keeling PW. Hypothalamic-pituitary-gut axis dysregulation in irritable bowel syndrome: plasma cytokines as a potential biomarker? Gastroenterology 2006;130:304-311.

25. Tache $Y$, Martinez V, Wang $L$, Million M. CRF1 receptor signaling pathways are involved in stress-related alterations of colonic function and viscerosensitivity: implications for irritable bowel syndrome. $\mathrm{Br} J$ Pharmacol 2004;141:1321-1330.

26. Sagami $Y$, Shimada $Y$, Tayama J, Nomura T, Satake M, Endo $Y$, Shoji T, Karahashi $K$, Hongo $M$, Fukudo $S$. Effect of a corticotropin releasing hormone receptor antagonist on colonic sensory and motor function in patients with irritable bowel syndrome. Gut 2004;53: 958-964.

27. Schiepers OJ, Wichers MC, Maes M. Cytokines and major depression. Prog Neuropsychopharmacol Biol Psychiatry 2005;29:201-217.

28. Leonard BE. The HPA and immune axes in stress: the involvement of the serotonergic system. Eur Psychiatry 2005;20 Suppl 3:S302-306.

29. Mills SC, Windsor AC, Knight SC. The potential interactions between polyunsaturated fatty acids and colonic inflammatory processes. Clin Exp Immunol 2005;142:216-228.

30. Calder PC. Polyunsaturated fatty acids, inflammation, and immunity. Lipids $2001 ; 36$ : 1007-1024.

31. Hibbeln JR, Linnoila M, Umhau JC, Rawlings R, George DT, Salem N, Jr. Essential fatty acids predict metabolites of serotonin and dopamine in cerebrospinal fluid among healthy control subjects, and early- and late-onset alcoholics. Biol Psychiatry 1998;44:235-242.

32. Calder PC. N-3 polyunsaturated fatty acids and inflammation: from molecular biology to the clinic. Lipids 2003;38:343-352.

33. Hibbeln JR, Umhau JC, Linnoila M, George DT, Ragan PW, Shoaf SE, Vaughan MR, Rawlings $R$, Salem N, Jr. A replication study of violent and nonviolent subjects: cerebrospinal fluid metabolites of serotonin and dopamine are predicted by plasma essential fatty acids. Biol Psychiatry 1998;44:243-249.

34. Gil A. Polyunsaturated fatty acids and inflammatory diseases. Biomed Pharmacother 2002 56:388-396.

35. Su KP, Huang SY, Chiu CC, Shen WW. Omega-3 fatty acids in major depressive disorder. A preliminary double-blind, placebo-controlled trial. Eur Neuropsychopharmacol 2003;13: 267-271.

36. Kilkens TO, Honig $A$, Maes $M$, Lousberg $R$, Brummer RJ. Fatty acid profile and affective dysregulation in irritable bowel syndrome. Lipids 2004;39:425-431.

37. Tyce GM. Origin and metabolism of serotonin. J Cardiovasc Pharmacol 1990;16 Suppl 3: S17.

38. Ortiz J, Artigas F, Gelpi E. Serotonergic status in human blood. Life Sci 1988:43:983-990.

39. Gershon MD. Serotonin and its implication for the management of irritable bowel syndrome. Rev Gastroenterol Disord 2003;3 Suppl 2:S25-34.

40. Wade PR, Chen J, Jaffe B, Kassem IS, Blakely RD, Gershon MD. Localization and function of a $5-\mathrm{HT}$ transporter in crypt epithelia of the gastrointestinal tract. J Neurosci 1996;16: 2352-2364.

41. Kema IP, de Vries EG, Muskiet FA. Clinical chemistry of serotonin and metabolites. J Chromatogr B Biomed Sci Appl 2000;747:33-48.

42. Fernstrom JD. Branched-chain amino acids and brain function. J Nutr 2005;135:1539S-46S.

43. Gershon MD. Review article: serotonin receptors and transporters - roles in normal and abnormal gastrointestinal motility. Aliment Pharmacol Ther 2004;20 Suppl 7:3-14.

44. Atkinson W, Lockhart S, Whorwell PJ, Keevil B, Houghton LA. Altered 5-hydroxytryptamine signaling in patients with constipation- and diarrhea-predominant irritable bowel syndrome. Gastroenterology 2006;130:34-43.

45. van Nieuwenhoven MA, Valks SD, Sobczak S, Riedel WJ, Brummer RJ. Acute tryptophan depletion slows gastric emptying in females. Br J Nutr 2004;91:351-355.

46. Kilkens TO, Honig A, van Nieuwenhoven MA, Riedel WJ, Brummer RJ. Acute tryptophan depletion affects brain-gut responses in irritable bowel syndrome patients and controls. Gut 2004:53:1794-1800. 
47. Jacobs BL, Fornal CA, Serotonin and behaviour. $A$ general hypothesis. In: Psychopharmacology: The Fourth Generation of Progress. New York: Raven Press Ltd.; 1995:461-469.

48. Blier P, Abbott FV. Putative mechanisms of action of antidepressant drugs in affective and anxiety disorders and pain. J Psychiatry Neurosci 2001;26:37-43.

49. Elhwuegi AS. Central monoamines and their role in major depression. Prog Neuropsychopharmacol Biol Psychiatry 2004;28:435-451.

50. Kilkens TO, Honig A, Rozendaal N, Van Nieuwenhoven MA, Brummer RJ. Serotonergic modulators in the treatment of irritable bowel syndrome - influence on psychiatric and gastrointestinal symptoms. Aliment Pharmacol Ther 2003;17:43-51.

51. Jackson JL, O'Malley PG, Tomkins $G$, Balden E, Santoro J, Kroenke K. Treatment of functional gastrointestinal disorders with antidepressant medications: a meta-analysis. Am J Med 2000;108:65-72.

52. Jordan D. Vagal control of the heart: central serotonergic (5-HT) mechanisms. Exp Physiol 2005:90:175-181.

53. Grubb BP, Karas BJ. The potential role of serotonin in the pathogenesis of neurocardiogenic syncope and related autonomic disturbances. J Interv Card Electrophysiol 1998;2:325-332.

54. Nahshoni E, Aravot D, Aizenberg D, Sigler M, Zalsman G, Strasberg B, Imbar S, Adier E Weizman A. Heart rate variability in patients with major depression. Psychosomatics 2004 ; 45:129-134.

55. Dinan TG. Serotonin and the regulation of hypothalamic-pituitary-adrenal axis function. Life Sci 1996;58:1683-1694.

56. John $C D$, Buckingham JC. Cytokines: regulation of the hypothalamo-pituitary-adrenocortical axis. Curr Opin Pharmacol 2003;3:78-84.

57. Raap DK, Van de Kar LD. Selective serotonin reuptake inhibitors and neuroendocrine function. Life Sci 1999:65:1217-1235.

58. Lowry CA, Moore FL. Regulation of behavioral responses by corticotropin-releasing factor Gen Comp Endocrinol 2006;146:19-27.

59. Basterzi AD, Aydemir C, Kisa C, Aksaray S, Tuzer V, Yazici K, Goka E. IL-6 levels decrease with SSRI treatment in patients with major depression. Hum Psychopharmacol 2005;20: 473-476

60. Choi JH, Zhang SY, Park KW, Cho YS, On BH, Lee MM, Park YB, Kim HS. The association between the T102C polymorphism of the HTR2A serotonin receptor gene and HDL cholesterol level in Koreans. J Biochem Mol Biol 2005;38:238-242.

61. Irvine EJ, Whitehead WE, Chey WD, Matsueda K, Shaw M, Talley NJ, Veldhuyzen van Zanten SJ. Design of treatment trials for functional gastrointestinal disorders. Gastroenterology 2006;130:1538-1551.

62. First MB, Spitzer RL, Gibbon M, J.B.W. W. Structured clinical interviews of DSM-IV Axis I disorders-clinicians version. Washington, DC: American Psychiatric Press; 1997.

63. Sheehan D. MINI International neuropsychiatric Interview: Tampa: University of South Florida; 1994

64. Beck AT, Steer RA, Brown GK. Manual for the Beck Depression Inventory-II. San Antonio, TX: The psychological Corporation; 1996.

65. Hamilton M. Development of a rating scale for primary depressive illiness. $\mathrm{Br} \mathrm{J}$ Soc Clin Psychol 1967;6:278-296.

66. Derogatis L. Confirmation of the dimcusional structure of the SCL-90: a study in construct validity. J of Clin Psychology 1977;33:981-989.

67. Zigmond AS, Snaith RP. The hospital anxiety and depression scale. Acta Psychiatr Scand 1983;67:361-370

68. Kellow JE. Principles of motility and sensation testing. Gastroenterol Clin North Am 2003;32: 733-750.

69. Mulak A. Testing of visceral sensitivity. J Physiol Pharmacol 2003;54 Suppl 4:55-72.

70. Bouin $M$, Plourde $V$, Boivin $M$, Riberdy $M$, Lupien $F$, Laganiere $M$, Verrier $P$, Poitras $P$. Rectal distention testing in patients with irritable bowel syndrome: sensitivity, specificity, and predictive values of pain sensory thresholds. Gastroenterology 2002;122:1771-1777. 
4 Chemer

71. van der Schaar PJ, Lamers CB, Masclee AA. The role of the barostat in human research and clinical practice. Scand J Gastroenterol Suppl 1999;230:52-63.

72. Hobson AR, Aziz Q. Brain imaging and functional gastrointestinal disorders: has it helped our understanding? Gut 2004;53:1198-1206.

73. Aziz Q. Thompson DG. Brain-gut axis in health and disease. Gastroenterology 1998;114: 559-578.

74. Andresen V, Poeltinger A, Tsrouya $C$, Bach D, Stroh A, Foerschler A, Georgiewa $P$, Schmidtmann $M$, van der Voort IR, Kobelt $P$, Zimmer $C$, Wiedenmann B, Klapp BF, Monnikes $\mathrm{H}$. Cerebral processing of auditory stimuli in patients with irritable bowel syndrome. World $\mathrm{J}$ Gastroenterol 2006;12:1723-1729.

75. Hobson AR, Hillebrand A. Independent component analysis of the EEG: is this the way forward for understanding abnormalities of brain-gut signalling? Gut 2006; 55: 597-600.

76. Hobson $A R, A z i z Q$. Central nervous system processing of human visceral pain in health and disease. News Physiol Sci 2003;18:109-114.

77. Kern MK, Shaker R. Cerebral cortical registration of subliminal visceral stimulation. Gastroenterology 2002;122:290-298.

78. Lawal A, Kern M, Sidhu H, Hofmann C, Shaker R. Novel evidence for hypersensitivity of visceral sensory neural circuitry in irritable bowel syndrome patients. Gastroenterology 2006; 130:26-33.

79. Sidhu $H$, Kern M, Shaker $R$. Absence of increasing cortical fMRI activity volume in response to increasing visceral stimulation in IBS patients. Am J Physiol Gastrointest Liver Physiol 2004;287:G425-435

80. Mathews $A$, Ridgeway $V$, Williamson DA. Evidence for attention to threatening stimuli in depression. Behav Res Ther 1996;34:695-705.

81. Mogg K, Bradley BP. Selective orienting of attention to masked threat faces in social anxiety. Behav Res Ther 2002;40:1403-1414.

82. Boyer MC, Compas BE, Stanger $C$, Colletti RB, Konik BS, Morrow SB, Thomsen AH. Attentional biases to pain and social threat in children with recurrent abdominal pain. J Pediatr Psychol 2006;31:209-220

83. Monair DM, Lorr M, Droppleman LF. Manual for the Profile of Mood States (POMS) manual. Educational and Industrial Testing Service. San Diego, CA; 1992.

84. Nikendei $C$, Dengler $W$, Wiedemann $G$, Pauli $P$. Selective processing of pain-related word stimuli in subclinical depression as indicated by event-related brain potentials. Biol Psychol 2005;70:52-60.

85. Burke $D$, Sundlof $G$, Wallin $G$. Postural effects on muscle nerve sympathetic activity in man. J Physiol 1977;272:399-414.

86. Hilz MJ, Dutsch M. Quantitative studies of autonomic function. Muscle Nerve $2006 ; 33: 6-20$

87. Emmanuel AV, Kamm MA. Laser Doppler flowmetry as a measure of extrinsic colonic innervation in functional bowel disease. Gut 2000;46:212-217.

88. van Orshoven NP, Andriesse Gl, van Schelven LJ, Smout AJ, Akkermans LM, Oey PL. Subtle involvement of the parasympathetic nervous system in patients with irritable bowel syndrome. Clin Auton Res 2006;16:33-39.

89. Lembo T, Plourde V, Shui Z, Fullerton S, Mertz H, Tache Y, Sytnik B, Munakata J, Mayer E. Effects of the corticotropin-releasing factor (CRF) on rectal afferent nerves in humans. Neurogastroenterol Motil 1996;8:9-18.

90. Elsenbruch S, Holtmann G, Oezcan D, Lysson A, Janssen O, Goebel MU, Schedlowski M. Are there alterations of neuroendocrine and cellular immune responses to nutrients in women with irritable bowel syndrome? Am J Gastroenterol 2004;99:703-710.

91. Pollmacher $T$, Haack $M$, Schuld $A$, Reichenberg A, Yirmiya R. Low levels of circulating inflammatory cytokines-Do they affect human brain functions? Brain Behav Immun 2002;16: 525-532.

92. Chadwick VS, Chen W, Shu D, Paulus B, Bethwaite $P$, Tie A, Wilson I. Activation of the mucosal immune system in irritable bowel syndrome. Gastroenterology 2002;122:1778-1783. 
93. Kristjansson $G$, Venge $P$, Wanders $A$, Loof $L$, Hallgren $R$. Clinical and subclinical intestinal inflammation assessed by the mucosal patch technique: studies of mucosal neutrophil and eosinophil activation in inflammatory bowel diseases and irritable bowel syndrome. Gut 2004; 53:1806-1812.

94. van der Veek PP, van den Berg M, de Kroon YE, Verspaget HW, Masclee AA. Role of tumor necrosis factor-alpha and interleukin-10 gene polymorphisms in irritable bowel syndrome. Am J Gastroenterol 2005;100:2510-2516.

95. Gonsalkorale WM, Perrey C, Pravica V, Whorwell PJ, Hutchinson IV. Interleukin 10 genotypes in irritable bowel syndrome: evidence for an inflammatory component? Gut 2003; 52:91-93.

96. Grimble RF, Howell WM, O'Reilly G, Turner SJ, Markovic O, Hirrell S, East JM, Calder PC. The ability of fish oil to suppress tumor necrosis factor alpha production by peripheral blood mononuclear cells in healthy men is associated with polymorphisms in genes that influence tumor necrosis factor alpha production. Am J Clin Nutr 2002;76:454-459.

97. Arab L. Biomarkers of fat and fatty acid intake. J Nutr 2003; 133 Suppl 3: 925S-932S.

98. Parker $G$, Gibson NA, Brotchie H, Heruc G, Rees AM, Hadzi-Pavlovic D. Omega-3 Fatty acids and mood disorders. Am J Psychiatry 2006;163:969-978.

99. Turnell DC, Cooper JD. Rapid assay for amino acids in serum or urine by pre-column derivatization and reversed-phase liquid chromatography. Clin Chem 1982;28:527-531.

100. Fernstrom JD. Diet-induced changes in plasma amino acid pattern: effects on the brain uptake of large neutral amino acids, and on brain serotonin synthesis. J Neural Transm Suppl 1979:55-67.

101. Atkinson W, Lockhart SJ, Houghton LA, Keevil BG. Validation of the measurement of low concentrations of 5-hydroxytryptamine in plasma using high performance liquid chromatography. J Chromatogr B Analyt Technol Biomed Life Sci 2006;832:173-176.

102. Lima L, Mata S, Urbina M. Allelic isoforms and decrease in serotonin transporter mRNA in lymphocytes of patients with major depression. Neuroimmunomodulation 2005;12:299-306.

103. Oreland L. Platelet monoamine oxidase, personality and alcoholism: the rise, fall and resurrection. Neurotoxicology 2004;25:79-89.

104. Oreland L, Wiberg A, Asberg M, Traskman L, Sjostrand L, Thoren $P$, Bertilsson $L$, Tybring $G$. Platelet MAO activity and monoamine metabolites in cerebrospinal fluid in depressed and suicidal patients and in healthy controls. Psychiatry Res 1981:4:21-29.

105. Lambert GW, Reid C, Kaye DM, Jennings GL, Esler MD. Effect of sunlight and season on serotonin turnover in the brain. Lancet 2002;360:1840-1842.

106. Coates MD, Mahoney CR, Linden DR, Sampson JE, Chen J, Blaszyk H, Crowell MD, Sharkey KA, Gershon MD, Mawe GM, Mose PL. Molecular defects in mucosal serotonin content and decreased serotonin reuptake transporter in ulcerative colitis and irritable bowel syndrome. Gastroenterology 2004;126:1657-1664.

107. Yatham LN, Steiner $M$. Neuroendocrine probes of serotonergic function: a critical review. Life Sci 1993;53:447-463.

108. Kapitany T, Schindl M, Schindler SD, Hesselmann B, Fureder T, Barnas C, Sieghart W, Kasper $\mathrm{S}$. The citalopram challenge test in patients with major depression and in healthy controls. Psychiatry Res 1999;88:75-88.

109. Modai I, Gibel A, Rauchverger B, Ritsner M, Klein E, Ben-Shachar D. Paroxetine binding in aggressive schizophrenic patients. Psychiatry Res 2000;94:77-81.

110. Booij $J$, de Win MM. Brain kinetics of the new selective serotonin transporter tracer [(123)l]ADAM in healthy young adults. Nucl Med Biol 2006;33 185-191.

111. McKie S, Del-Ben C, Elliott R, Williams S, Del Vai N, Anderson I, Deakin JF. Neuronal effects of acute citalopram detected by pharmacoMRI. Psychopharmacology (Berl) 2005;180: 680-686.

112. Serretti $A$, Benedetti $F$, Zanardi $R$, Smeraldi $E$. The influence of Serotonin Transporter Promoter Polymorphism (SERTPR) and other polymorphisms of the serotonin pathway on the efficacy of antidepressant treatments. Prog Neuropsychopharmacol Biol Psychiatry 2005;29: 1074-1084. 
$50 \mid$ Chapter 2

113. Eichhammer $P$, Langguth $B$, Wiegand $R$, Kharraz $A$, Frick $U$, Hajak $G$. Allelic variation in the serotonin transporter promoter affects neuromodulatory effects of a selective serotonin transporter reuptake inhibitor (SSRI). Psychopharmacology (Berl) 2003;166:294-297.

114. Serretti A, Cusin C, Rossini D, Artioli P, Dotoli D, Zanardi R. Further evidence of a combined effect of SERTPR and TPH on SSRIs response in mood disorders. Am J Med Genet B Neuropsychiatr Genet 2004;129:36-40.

115. Kim HJ, Camilleri M, Carlson PJ, Cremonini F, Ferber I, Stephens D, McKinzie S, Zinsmeister $A R$, Urrutia R. Association of distinct alpha(2) adrenoceptor and serotonin transporter polymorphisms with constipation and somatic symptoms in functional gastrointestinal disorders. Gut 2004:53:829-837.

116. Pata $C$, Erdal $M E$, Derici $E$, Yazar $A$, Kanik $A$, Ulu $O$. Serotonin transporter gene polymorphism in irritable bowel syndrome. Am J Gastroenterol 2002;97:1780-1784.

117. Yeo $A$, Boyd $P$, Lumsden $S$, Saunders $T$, Handley $A$, Stubbins $M$, Knaggs $A$, Asquith $S$, Taylor I, Bahari B, Crocker N, Rallan R, Varsani S, Montgomery D, Alpers DH, Dukes GE, Purvis I, Hicks GA. Association between a functional polymorphism in the serotonin transporter gene and diarrhoea predominant irritable bowel syndrome in women. Gut 2004; 53:1452-1458.

118. Lee DY, Park H, Kim WH, Lee SI, Seo YJ, Choi YC. Serotonin transporter gene polymorphism in healthy adults and patients with irritable bowel syndrome. Korean $\mathrm{J}$ Gastroenterol 2004:43:18-22.

119. Camilleri M. Is there a SERT-ain association with IBS? Gut 2004;53:1396-1399.

120. Young SN, Smith SE, Pihl RO, Ervin FR. Tryptophan depletion causes a rapid lowering of mood in normal males. Psychopharmacology 1985;87:173-177.

121. Carpenter LL, Anderson GM, Pelton GH, Gudin JA, Kirwin PD, Price LH, Heninger GR, McDougle CJ. Tryptophan depletion during continuous CSF sampling in healthy human subjects. Neuropsychopharmacology 1998;19:26-35.

122. Young $S N$, Leyton $M$, Benkelfat $C$. Pet studies of serotonin synthesis in the human brain. Adv Exp Med Biol 1999:467:11-18.

123. Nishizawa $S$, Benkelfat $C$, Young SN, Leyton M, Mzengeza $S$, de Montigny $C$, Blier $P$, Diksic $M$. Differences between males and females in rates of serotonin synthesis in human brain. Proc Natl Acad Sci U S A 1997; $94: 5308-5313$.

124. Booij L, Vam der Does AJ, Haffmans PM, Spinhoven P, McNally RJ. Acute tryptophan depletion as a model of depressive relapse: Behavioural specificity and ethical considerations. Br J Psychiatry 2005;187:148-154.

125. Anderson IM, Parry-Billings $M$, Newsholme EA, Fairburn CG, Cowen PJ. Dieting reduces plasma tryptophan and alters brain 5-HT function in women. Psychol Med 1990;20:785-791.

126. Praschak-Rieder N, Wilson AA, Hussey D, Carella A, Wei C, Ginovart N, Schwarz MJ, Zach $J$, Houle $S$, Meyer $\mathrm{JH}$. Effects of tryptophan depletion on the serotonin transporter in healthy humans. Biol Psychiatry 2005;58:825-830.

127. Smith KA, Clifford EM, Hockney RA, Clark DM, Cowen PJ. Effect of tryptophan depletion on mood in male and female volunteers: A pilot study. Human Psychopharmacology 1997;12: 111-117.

128. Klaassen T, Riedel WJ, van Someren A, Deutz NE, Honig A, van Praag HM. Mood effects of 24-hour tryptophan depletion in healthy first-degree relatives of patients with affective disorders. Biol Psychiatry 1999;46:489-497.

129. Neumeister A, Konstantinidis A, Stastny J, Schwarz MJ, Vitouch O, Willeit M, PraschakRieder N, Zach J, de Zwaan M, Bondy B, Ackenheil M, Kasper S. Association between serotonin transporter gene promoter polymorphism (5HTTLPR) and behavioral responses to tryptophan depletion in healthy women with and without family history of depression. Arch Gen Psychiatry 2002;59:613-620.

130. Booij L, Van Der Does AJ, Riedel WJ. Monoamine depletion in psychiatric and healthy populations: review. Mol Psychiatry 2003;8:951-973

131. Miller HE, Deakin JF, Anderson IM. Effect of acute tryptophan depletion on CO2-induced anxiety in patients with panic disorder and normal volunteers. $\mathrm{Br} J$ Psychiatry 2000;176: 182-188. 
132. Riedel WJ. Cognitive changes after acute tryptophan depletion: what can they tell us? Psychol Med 2004;34:3-8.

133. Klaassen T, Riedel WJ, Deutz NE, Van Praag HM. Mood congruent memory bias induced by tryptophan depletion. Psychol Med 2002;32:167-172.

134. Booij L, Swenne CA, Brosschot JF, Haffmans PM, Thayer JF, Van der Does AJ. Tryptophan Depletion Affects Heart Rate Variability and Impulsivity in Remitted Depressed Patients with a History of Suicidal Ideation. Biol Psychiatry 2006.

135. Harrison BJ, Olver JS, Norman TR, Nathan PJ. Effects of serotonin and catecholamine depletion on interleukin-6 activation and mood in human volunteers. Hum Psychopharmacol 2002;17:293-297.

136. Ravindran AV, Griffiths J, Merali $Z$, Knott $V J$, Anisman $H$. Influence of acute tryptophan depletion on mood and immune measures in healthy males. Psychoneuroendocrinology 1999;24:99-113.

137. Stastny J, Konstantinidis A, Schwarz MJ, Rosenthal NE, Vitouch O, Kasper $S$, Neumeister $A$. Effects of tryptophan depletion and catecholamine depletion on immune parameters in patients with seasonal affective disorder in remission with light therapy. Biol Psychiatry 2003; 53:332-337.

138. Hyttel J. Citalopram--pharmacological profile of a specific serotonin uptake inhibitor with antidepressant activity. Prog Neuropsychopharmacol Biol Psychiatry 1982;6:277-295.

139. Bezchlibnyk-Butler K, Aleksic I, Kennedy SH. Citalopram--a review of pharmacological and clinical effects. J Psychiatry Neurosci 2000;25:241-254.

140. Lotrich FE, Bies R, Muldoon MF, Manuck SB, Smith GS, Pollock BG. Neuroendocrine response to intravenous citalopram in healthy control subjects: pharmacokinetic influences. Psychopharmacology (Berl) 2005;178:268-275.

141. Seifritz E, Baumann $P$, Muller MJ, Annen O, Amey M, Hemmeter $U$, Hatzinger M, Chardon $F$, Holsboer-Trachsler E. Neuroendocrine effects of a $20-\mathrm{mg}$ citalopram infusion in healthy males. A placebo-controlled evaluation of citalopram as 5-HT function probe. Neuropsychopharmacology 1996;14:253-263.

142. Carpenter LL, Anderson GM. Acute changes in CSF 5-HIAA following oral paroxetine challenge in healthy humans. Neuropsycho Pharmacology 2003;28:339-347.

143. Kilkens TO, Honig A, Fekkes D, Brummer RJ. The effects of an acute serotonergic challenge on brain-gut responses in irritable bowel syndrome patients and controls. Aliment Pharmacol Ther 2005;22:865-874.

144. Blier P. The pharmacology of putative early-onset antidepressant strategies. Eur Neuropsychopharmacol 2003;13:57-66.

145. Harmer CJ, Shelley NC, Cowen PJ, Goodwin GM. Increased positive versus negative affective perception and memory in healthy volunteers following selective serotonin and norepinephrine reuptake inhibition. Am J Psychiatry 2004;161:1256-1263.

146. Zobel AW, Schulze-Rauschenbach S, von Widdern OC, Metten M, Freymann N, Grasmader $K$, Pfeiffer $U$, Schnell S, Wagner $M$, Maier $W$. Improvement of working but not declarative memory is correlated with HPA normalization during antidepressant treatment. J Psychiatr Res 2004;38:377-383.

147. Mogg K, Baldwin DS, Brodrick P, Bradley BP. Effect of short-term SSRI treatment on cognitive bias in generalised anxiety disorder. Psychopharmacology (Berl) 2004;176:466-470.

148. Siepmann M, Grossmann J, Muck-Weymann M, Kirch W. Effects of sertraline on autonomic and cognitive functions in healthy volunteers. Psychopharmacology (Berl) 2003;168:293-298.

149. Willeit M, Praschak-Rieder N, Neumeister A, Pirker W, Asenbaum S, Vitouch O, Tauscher J, Hilger E, Stastny J, Brucke T, Kasper S. [123l]-beta-CIT SPECT imaging shows reduced brain serotonin transporter availability in drug-free depressed patients with seasonal affective disorder. Biol Psychiatry 2000;47:482-489.

150. Smith GS, Ma $Y$, Dhawan $V$, Gunduz $H$, Carbon $M$, Kirshner $M$, Larson J, Chaly $T$, Belakhleff A, Kramer E, Greenwald B, Kane JM, Laghrissi-Thode F, Pollock BG, Eidelber D. Serotonin modulation of cerebral glucose metabolism measured with positron emission tomography (PET) in human subjects. Synapse 2002;45:105-112. 
52 Chapter 2

151. Peter H, Tabrizian S, Hand I. Serum cholesterol in patients with obsessive compulsive disorder during treatment with behavior therapy and SSRI or placebo. Int J Psychiatry Med 2000;30:27-39.

152. Gorard DA, Dewsnap PA, Medbak SH, Perry LA, Libby GW, Farthing MJ. Central 5hydroxytryptaminergic function in irritable bowel syndrome. Scand J Gastroenterol 1995;30: 994-999.

153. Tack J, Broekaert D, Corsetti M, Fischler B, Janssens J. Influence of acute serotonin reuptake inhibition on colonic sensorimotor function in man. Aliment Pharmacol Ther 2006; 23:265-274.

154. Tack J, Broekaert D, Fischler B, Van Oudenhove L, Gevers A, Janssens J. A controlled cross-over study of the selective serotonin reuptake inhibitor citalopram in irritable bowel syndrome. Gut 2006;55:1095-1103.

155. Creed F, Fernandes L, Guthrie E, Palmer S, Ratcliffe J, Read N, Rigby C, Thompson D Tomenson B. The cost-effectiveness of psychotherapy and paroxetine for severe irritable bowel syndrome. Gastroenterology 2003;124:303-317.

156. Kuiken SD, Tytgat GN, Boeckxstaens GE. The selective serotonin reuptake inhibitor fluoxetine does not change rectal sensitivity and symptoms in patients with irritable bowel syndrome: a double blind, randomized, placebo-controlled study. Clin Gastroenterol Hepatol 2003;1:219-228.

157. Tabas $G$, Beaves $M$, Wang J, Friday $P$, Mardini $H$, Arnold $G$. Paroxetine to treat irritable bowel syndrome not responding to high-fiber diet: a double-blind, placebo-controlled trial. Am J Gastroenterol 2004;99:914-920.

158. Vahedi H, Merat S, Rashidioon A, Ghoddoosi A, Malekzadeh R. The effect of fluoxetine in patients with pain and constipation-predominant irritable bowel syndrome: a double-blind randomized-controlled study. Aliment Pharmacol Ther 2005;22:381-385.

159. Camilleri M. Serotonergic modulation of visceral sensation: lower gut. Gut 2002;51 Suppl 1: i81-86.

160. Stancak A, Polacek H, Vrana J, Rachmanova R, Hoechstetter K, Tintra J, Scherg M. EEG source analysis and fMRI reveal two electrical sources in the fronto-parietal operculum during subepidermal finger stimulation. Neuroimage 2005;25:8-20.

161. Chang L, Toner BB, Fukudo S, Guthrie E, Locke GR, Norton NJ, Sperber AD. Gender, age, society, culture, and the patient's perspective in the functional gastrointestinal disorders. Gastroenterology 2006;130:1435-1446.

162. Sloots CE, Felt-Bersma RJ, Cuesta MA, Meuwissen SG. Rectal visceral sensitivity in healthy volunteers: influences of gender, age and methods. Neurogastroenterol Motil 2000;12: 361-368.

163. Lagier E, Delvaux M, Velias B, Fioramonti J, Bueno L, Albarede JL, Frexinos J. Influence of age on rectal tone and sensitivity to distension in healthy subjects. Neurogastroenterol Motil 1999;11:101-107.

164. Houghton $L A$, Lea $R$, Jackson $N$, Whorwell PJ. The menstrual cycle affects rectal sensitivity in patients with irritable bowel syndrome but not healthy volunteers. Gut 2002;50:471-474.

165. Naliboff BD, Berman S, Chang L, Derbyshire SW, Suyenobu B, Vogt BA, Mandelkern M, Mayer EA. Sex-related differences in IBS patients: central processing of visceral stimuli. Gastroenterology 2003;124:1738-1747.

166. Dinan TG, Barry S, Ahkion S, Chua A, Keeling PW. Assessment of central noradrenergic functioning in irritable bowel syndrome using a neuroendocrine challenge test. J Psychosom Res 1990;34:575-580.

167. Stahl S, Briley M. Understanding pain in depression. Hum Psychopharmacol 2004;19 Suppl 1: S9-S13.

168. Fernstrom JD, Wurtman RJ, Hammarstrom-Wiklund B, Rand WM, Munro HN, Davidson CS. Diurnal variations in plasma concentrations of tryptophan, tryosine, and other neutral amino acids: effect of dietary protein intake. Am J Clin Nutr 1979;32:1912-1922. 
169. Markus CR, Olivier B, Panhuysen GE, Van Der Gugten J, Alles MS, Tuiten A, Westenberg $H G$, Fekkes $D$, Koppeschaar HF, de Haan EE. The bovine protein alpha-lactalbumin increases the plasma ratio of tryptophan to the other large neutral amino acids, and in vulnerable subjects raises brain serotonin activity, reduces cortisol concentration, and improves mood under stress. Am J Clin Nutr 2000;71:1536-1544.

170. Murphy SE, Longhitano C, Ayres RE, Cowen PJ, Harmer CJ. Tryptophan supplementation induces a positive bias in the processing of emotional material in healthy female volunteers. Psychopharmacology (Berl) 2006;187:121-130. 


\section{Chapter 3}

Systematic review: serotonergic modulators in the treatment of irritable bowel syndrome influence on psychiatric and gastrointestinal symptoms 


\section{Abstract}

\section{Background}

Both central and peripheral serotonergic modulators are used in the treatment of irritable bowel syndrome (IBS). The majority of IBS patients presenting to a gastroenterologist demonstrate affective dysregulation. Serotonin $(5-H T)$ may play a regulatory role in both gastrointestinal motility and sensitivity, as well as in affective dysregulation, in IBS.

Aim

To analyse, systematically, randomised controlled trials studying the influence of 5-HT modulators on both gastrointestinal and psychiatric symptoms in IBS, in order to elucidate baseline IBS symptomatology and possible differential effects of 5-HT modulation on this symptomatology.

Methods

A standardized qualitative analysis was performed of studies investigating the influence of 5-HT modulators on both gastrointestinal and psychiatric symptoms in IBS using a blind review approach. The studies were ranked according to their total quality score (maximum 100 points).

\section{Results}

Eleven studies fulfilled entry criteria, six of which scored above 55 points. An association between gastroenterological and psychiatric changes was present in five of the six studies.

\section{Conclusions}

The results strengthen the serotonergic association between gastroenterological and psychiatric symptoms. Adjusted guidelines for combined gastrointestinal and psychiatric assessments are recommended in order to further elucidate the serotonergic interaction between gastrointestinal and psychiatric symptoms. 


\section{Introduction}

The pathophysiology of irritable bowel syndrome (IBS) is poorly understood. A multi-component conceptual model of IBS has been postulated, involving physiological, affective, cognitive and behavioural factors ${ }^{1}$. The concept that IBS is a neurological bowel disease rather than a functional disease is gaining ground ${ }^{2}$. Interaction between the gut and the central nervous system (CNS) plays a major role in the pathophysiology of IBS symptoms. This biopsychosocial conceptualisation is called the 'brain-gut axis', a theoretical model describing the bi-directional neural pathway linking cognitive and emotional centres in the brain to neuroendocrine centres, the enteric nervous system (ENS) and the immune system ${ }^{3}$. According to this model, IBS (and other functional gastrointestinal disorders) results from dysregulation of brain-gut neuroenteric systems.

The high co-occurrence of IBS with psychiatric symptoms, in particular affective dysregulation, has been reported in the gastroenterological setting. The most frequent comorbid psychiatric disorders include: mood disorders (major depression and dysthymic disorder), anxiety disorders (panic and generalized anxiety disorder) and somatoform disorders ${ }^{4-9}$. The prevalence of these psychiatric disorders in IBS patients seen in the gastroenterological setting is estimated between 40 and $90 \%{ }^{6,10,11}$. In patients with inflammatory bowel disease, a disorder which demonstrates similar chronic intermittent abdominal symptoms as IBS, a much lower prevalence of psychiatric comorbidity of about $20 \%$ has been found ${ }^{12,13}$. Hence, the high rates of psychiatric comorbidity in IBS patients indicate that affective symptoms may be a specific and integral part of IBS, rather than a non-specific syndrome related to a chronic intestinal disease ${ }^{5,14}$. This presumption fits well with the proposed 'brain-gut' concept. A possible common denominator involved in the pathophysiology of IBS and affective dysregulation is serotonin (5-HT), as both gut motility and affective regulation are specifically mediated by this neurotransmitter. Manipulation of serotonergic activity by the application of antidepressants, selective serotonin reuptake inhibitors (SSRIs), tricyclic antidepressants (TCAs) and other 5-HT modulators (5-HT 3 and $5-\mathrm{HT}_{4}$ agonists and antagonists), has been widely used in the treatment of both affective disorders and IBS ${ }^{15-20}$. In contrast with former reviews, the purpose of this article is to review only studies which describe both baseline gastrointestinal and psychiatric symptoms and the influence of 5-HT modulators on both sets of symptoms.

In accordance with the 'brain-gut' concept, a change in 5-HT activity will affect the 'brain-gut axis' at any level. We hypothesize that IBS, presented in the gastroenterological setting, is characterised by concurrent baseline gastrointestinal and affective dysregulation, and that treatment of IBS with 
serotonergic modulators, in line with the 'brain-gut concept', will influence both gastrointestinal and psychiatric symptoms simultaneously.

\section{Materials and methods}

\section{Literature search}

We searched MEDLINE (1966 to April 2002), Psychinfo (1966 to April 2002), EMBASE (1984 to April 2002), and the Cochrane Library (2002, issue 2) using the Cochrane Database of Systematic Reviews, Database of Abstracts of Reviews of Effectiveness and The Cochrane Controlled Trials Register. The following key words were used: colon(ic) disease(s)/disorder(s) (functional), irritable colon/bowel (syndrome), gastrointestinal disorders/system. All citations in the papers identified in this way were checked in order to complete our set of publications.

\section{Inclusion criteria}

The papers had to meet all of the following criteria: (i) the patients examined in the study had to be diagnosed with the IBS symptom complex; (ii) one of the aims of the study was the assessment of the efficacy of a serotonergic modulator, e.g. TCA, SSRI, 5- $\mathrm{HT}_{3 / 4}$ (ant)agonist, on both gastrointestinal and psychiatric symptoms; (iii) adequate description of randomisation; (iv) doubleblinding; ( $v$ ) controlled design with placebo and a known serotonergic modulator; (vi) for practical reasons, the study had to be written in English, French, German or Dutch; (vii) a full report. Letters and abstracts were excluded.

\section{Methodological quality assessment}

The methodological quality of the studies was assessed according to standardized methodological criteria ${ }^{21}$. An a priori cut-off of 55 was set, similar to previous studies ${ }^{22,23}$. This score is regarded as representing an adequate quality of the paper. Before examining the retrieved literature, three of the authors (TOCK, AH, R-JMB) designed a list of 47 criteria concerning 11 methodological aspects of a pharmacological study (Table 3.1 and Appendix 3.A). Most of the methodological criteria were adapted from the "Maastricht Methodological Criteria List" ${ }^{21,22}$. These criteria concerned three aspects of the study: (i) internal validity (e.g. randomisation, blinding, loss to follow-up); (ii) precision of the study (sample size and variability); and (iii) relevance of choices with respect to the study population, interventions and outcome parameters $^{21}$. 
Table 3.1 Methodological scores of 11 studies.

\begin{tabular}{|c|c|c|c|c|c|c|c|c|c|c|c|c|c|}
\hline \multicolumn{2}{|c|}{ Criterion } & A & B & C & D & $E$ & $\mathrm{~F}$ & G & $H$ & 1 & $\mathrm{~J}$ & $\mathrm{~K}$ & Total \\
\hline \multicolumn{2}{|c|}{$\begin{array}{l}\text { Maximum Score } \\
\text { Included studies }\end{array}$} & 25 & 4 & 6 & 6 & 4 & 6 & 6 & 20 & 4 & 9 & 10 & 100 \\
\hline \multirow{2}{*}{\multicolumn{2}{|c|}{$\begin{array}{ll}1 & \text { Schutze et al. }{ }^{24} \\
2 & \text { Watson et al. } \\
& \text { Camilleri et al. }{ }^{27} \text { (S3BA3002) }\end{array}$}} & 18 & 4 & 6 & 3 & 3 & 4 & 4 & 20 & 0 & 5 & 10 & 77 \\
\hline & & 17 & 4 & 6 & 4 & 3 & 4 & 4 & 17 & 3 & 3 & 10 & 75 \\
\hline 3 & Van Outryve et al. ${ }^{25}$ & 19 & 4 & 6 & 4 & 3 & 4 & 4 & 14 & 0 & 5 & 8 & 71 \\
\hline \multirow{2}{*}{$\begin{array}{l}4 \\
5\end{array}$} & Greenbaum et al. ${ }^{26}$ & 20 & 4 & 3 & 6 & 3 & 1 & 1 & 14 & 0 & 3 & 8 & 63 \\
\hline & Watson et al. ${ }^{27}$ (S3BA3001) & 15 & 4 & 3 & 4 & 0 & 4 & 4 & 14 & 0 & 3 & 10 & 61 \\
\hline \multirow{2}{*}{$\begin{array}{l}6 \\
7\end{array}$} & Maxton et al. ${ }^{28}$ & 13 & 4 & 3 & 5 & 4 & 1 & 1 & 17 & 0 & 0 & 8 & 56 \\
\hline & 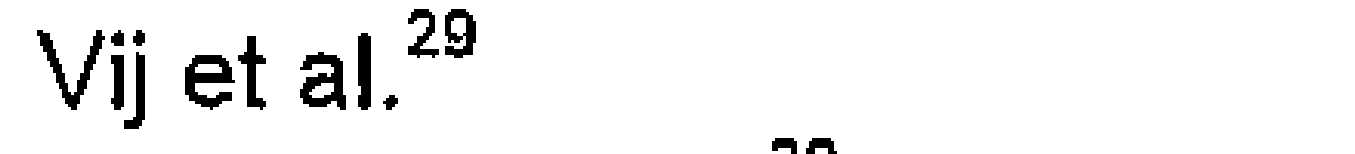 & 10 & 4 & 2 & 1 & 3 & 3 & 3 & 17 & 0 & 3 & 8 & 54 \\
\hline $\begin{array}{l}7 \\
8\end{array}$ & Bergmann et al. ${ }^{30}$ & 10 & 4 & 2 & 2 & 3 & 4 & 4 & 11 & 4 & 0 & 8 & 52 \\
\hline$\theta$ & Myren et al. ${ }^{33}$ & 10 & 4 & 2 & 5 & 4 & 1 & 1 & 17 & 0 & 0 & 8 & 52 \\
\hline & Heefner et al. ${ }^{34}$ & 10 & 4 & 2 & 3 & 3 & 5 & 5 & 11 & 0 & 0 & 6 & 49 \\
\hline & 11 Myren et al. ${ }^{31}$ & 10 & 4 & 3 & 4 & 0 & 3 & 3 & 14 & 0 & 0 & 6 & 47 \\
\hline \multicolumn{2}{|c|}{ Sum score 11 studies } & 152 & 44 & 38 & 41 & 29 & 34 & 34 & 166 & 11 & 22 & 90 & 657 \\
\hline \multicolumn{2}{|c|}{ Mean } & 14 & 4 & 3 & 4 & 3 & 3 & 3 & 15 & 0.6 & 2 & 8 & 60 \\
\hline \multicolumn{2}{|c|}{$\%$ of maximum score } & 55 & 100 & 58 & 62 & 66 & 52 & 52 & 75 & 16 & 22 & 82 & 60 \\
\hline
\end{tabular}

$A$, selection and restriction; $B$, treatment allocation; $C$, study size; $D$, prognostic comparability; $E$, drop outs/follow-up; $F$, intervention/experimental; $G$, intervention/placebo; $H$, outcome measures; l, follow-up period; $J$, description of side-effects in each group; $K$, analyses and presentation of data.

A maximum score of 100 was determined beforehand and important criteria received relatively higher maximum scores. All studies were scored for each of the 47 criteria. In order to prevent bias, reviewed papers were blinded for author, name and location of institute, journal and year of publication. Two other authors (NR, MAvN), who were not involved in the design of the methodological criteria, scored the 47 criteria. Disagreements between the reviewers (NR, MAvN), were identified and subsequently discussed during a consensus meeting. If disagreements were not resolved during this meeting, $\mathrm{AH}$ and R-JMB made a final judgement. The final scores for the articles were summed. This resulted in a hierarchical list in which higher scores indicated studies that were considered to be of a better methodological quality.

\section{Results}

\section{Literature search}

The literature search resulted in the identification of 19 publications ${ }^{24-42}$. Subsequently, nine publications were excluded. Six of these did not assess the influence on both gastrointestinal and psychiatric symptoms before and after treatment ${ }^{36,37,39-42}$. Two sets of two duplicate publications, based on the same data, were identified ${ }^{26,31,32,38}$. One publication was excluded because a combined anxiolytic/antidepressant was applied ${ }^{35}$. Both duplicate studies were 
$60 \mid$ Chapter 3

assessed following the set of methodological criteria; the paper with the lowest methodological score was excluded ${ }^{38}$. The two identical publications of Myren et al. ${ }^{31,32}$ received an equal methodological score; the reference used in this review is the English version ${ }^{32}$. Of the ten publications left, the publication of Watson et al. reported on two different studies (denoted S3BA3001 and S3BA3002), which were reviewed separately ${ }^{27}$. From one of these two studies (S3BA3002), more detailed information was published in another article by Camilleri et al. ${ }^{43}$. This article was also used to review study S3BA3002. Eleven studies met the inclusion criteria and were included in this review ${ }^{24-31,33,34,43}$ (see also Table 3.2).

\section{Blinding procedure}

The raters (NR, MAvN) agreed on the score for 412 of the 517 items $(80 \%)$ before the consensus meeting. In order to assess whether blinding of the articles was successful, the raters were asked for the possible name of the author, journal, year of publication and the place and institute for each article. Blinding of the articles was successful for $100 \%$ of articles with regard to authors, $91 \%$ with regard to journal, $73 \%$ with regard to year of publication and $100 \%$ with regard to place and institute. After the consensus meeting, only eleven items were left to be scored by AH and R-JMB.

\section{Methodological rating}

The results of the methodological assessment are presented in Table 3.1, in which articles are presented according to their total 'quality score': Specific methodological shortcomings are indicated. The methodological score ranged from 47 to 77 (mean, 60). Of the eleven studies, six studies received a score

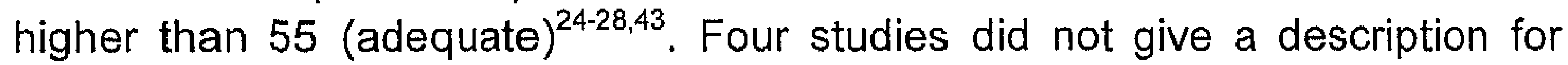
diagnosing IBS. Five studies applied the Rome or Manning criteria for the diagnosis IBS. None of the studies described a psychiatric diagnosis and, in three studies, a psychiatric diagnosis was an exclusion criterion. Eight studies made a subdivision of IBS into diarrhoea- and constipation-predominant subtypes. Eight studies described the level of gastrointestinal symptoms and all studies described the level of psychiatric symptoms at baseline. To assess the level of psychiatric symptoms, ten different subjective symptom checklists, mostly not comparable, on affective dysregulation were used (Table 3.2). Only four studies gave an adequate description of comorbidity and co-medication, and three studies performed a power analysis. All studies had a group size of 15 subjects or more. Prognostic comparability between the placebo and experimental study populations was poorly described, except for comparable demographics and baseline outcome measures. Nine studies described the number of drop-outs. In eight studies, the duration of the intervention was more 
than eight weeks. Drug compliance was described only once ${ }^{34}$. Nine studies used global outcome parameters; only five studies used standardized global outcome measures. In three papers, a differentiation between primary and secondary gastroenterological outcome measures was made ${ }^{24,28,43}$. A follow-up period ranging from four weeks to one year was described in two studies ${ }^{30,43}$. Six of the studies measured possible side-effects, but none of these studies used specific systematic questionnaires. Adequate statistical methods were used in ten studies. In only three studies an intention-to-treat analysis was performed ${ }^{24,27,43}$.

\section{Efficacy}

The following results were derived from the six studies which received an adequate methodological quality score. Two studies compared cisapride (5- $\mathrm{HT}_{4}$ agonist: enhances acetylcholine release; in addition, 5- $\mathrm{HT}_{3}$ antagonist $)^{24,25}$, two alosetron (5-HT 3 antagonist) ${ }^{27,43}$, one ondansetron $\left(5-\mathrm{HT}_{3}\right.$

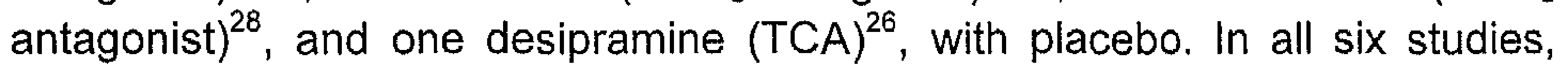
baseline gastroenterological symptomatology, compatible with a diagnosis of IBS, was present in all patients. Although two studies excluded psychiatric disorders ${ }^{24,25}$ in all six studies relevant psychopathological data were present at baseline. At baseline, the studies of Schutze et al. and Van Outryve et al. measured the presence of nervousness, tension, anxiety and feeling depressed on a $0-3$ scale $^{24,25}$. In these studies, nervousness was present in $50-76 \%$ of patients, tension in $25-48 \%$, anxiety in $21-45 \%$, and depression in $14-33 \%$. The Irritable Bowel Syndrome Quality of Life Questionnaire (IBSQOL) used in studies S3BA3001 and S3BA3002, showed, at baseline, decreased scores compared to a control group without IBS (but with other gastrointestinal conditions) for the subscales emotional health, mental health, sleep, energy and social role ${ }^{44}$. The overall score on the IBSQOL varied between 48 and 77 (control group, 88-98)(numbers estimated from tables), with 100 representing the best possible quality of life score. Greenbaum et al. reported a mean score of 45.2 on the Brief Psychiatric Rating Scales (BPRS)(no cut-off score for pathology/non-pathology) and 26.0 on the Hamilton Depression Rating Scale (HDRS) (0-7, no depression; 8-17, mild depression; 18-25, moderate depression; $>26$, severe depression ${ }^{26}$. The Hospital Anxiety and Depression Scale (HADS), used in the study of Maxton et al., was positive for anxiety (mean, 9.2) and negative for depression (mean, 5.9) at baseline ( $\geq 8$ indicating possible pathology $)^{28}$.

In five of the six studies, gastroenterological symptoms improved ${ }^{25-28,43}$. In four of these studies, a simultaneous improvement of psychiatric symptoms was reported $^{25-27,43}$. Two studies reported a partial improvement of psychiatric symptoms: Van Outryve et al. reported an improvement of nervousness only ${ }^{25}$, and Watson et al. S3BA3002 reported no improvement in mental health ${ }^{27}$. 
$62 \mid$ Chapter 3

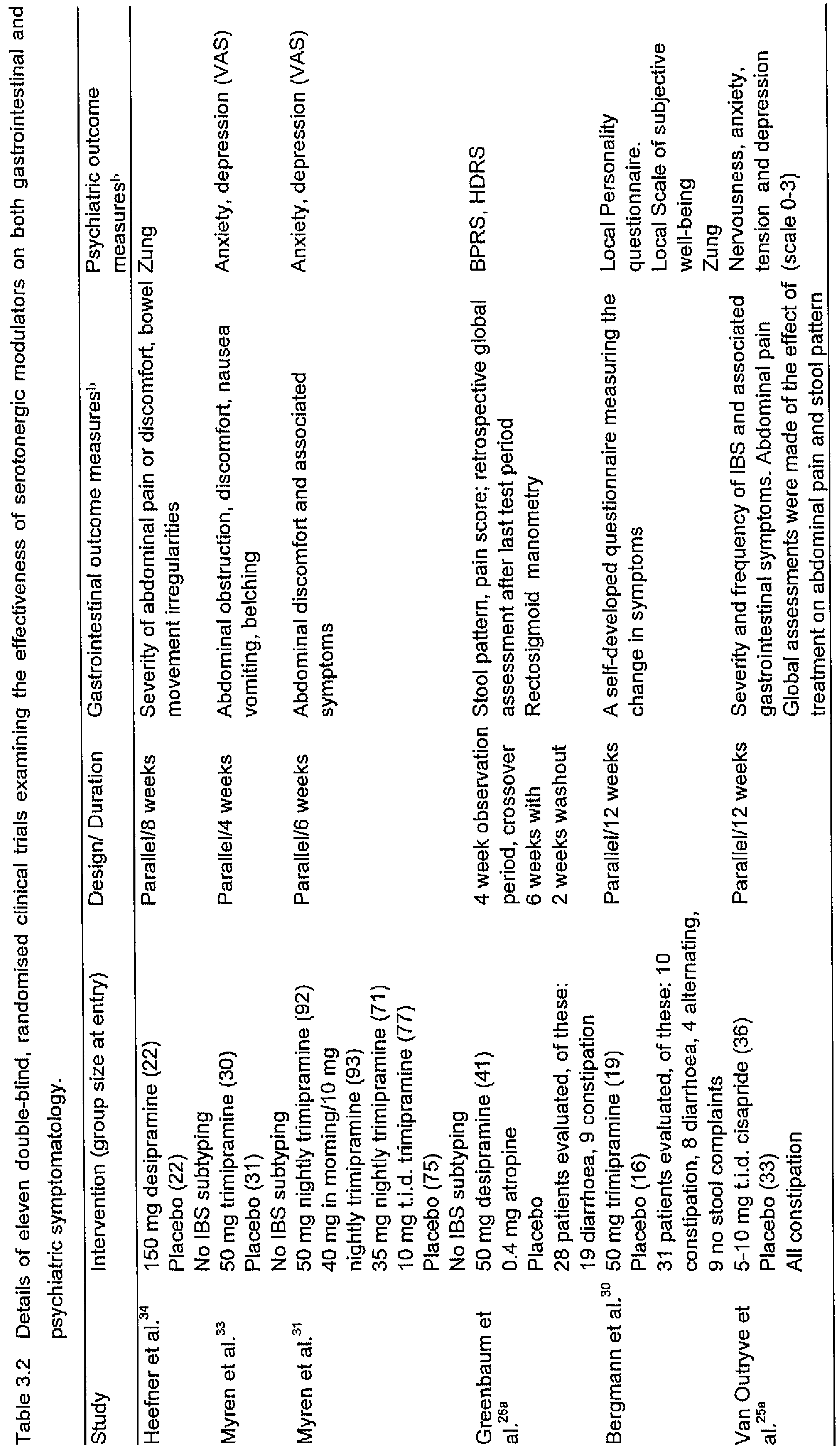


Review: Serotonergic modulation in irritable bowel syndrome $\mid 63$

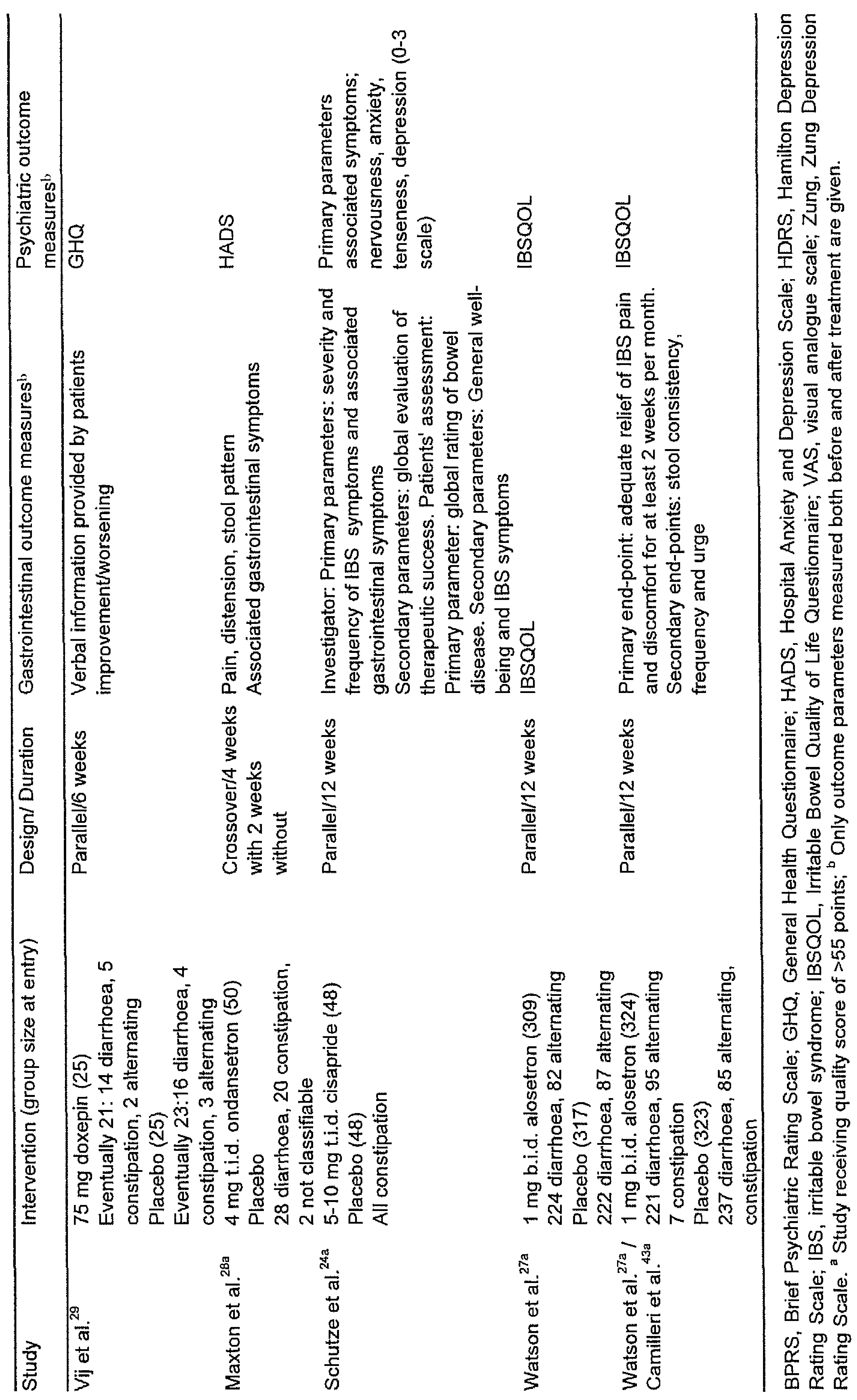


64 Chapter 3

The IBSQOL has a Minimum Meaningful Difference (MMD) determined for each scale. A MMD was present for the subscale social role in study S3BA3002 and the subscales social role and energy in study S3BA3001. Maxton et al. reported no significant improvement for abdominal pain or distension ${ }^{28}$. However, a significant improvement in bowel frequency and improved stool was found in the diarrhoea-predominant IBS patients, as well as some of the associated symptoms (postprandial discomfort, heartburn and flatulence). Psychiatric symptoms, however, did not improve. The study of Schutze et al. showed no significant improvement in gastroenterological (except for stool passage) and psychiatric symptoms ${ }^{24}$. From these results, no possible influence of IBS subtyping can be made. Statistical pooling of the results of the six studies with a methodological score of 55 or above was not possible due to the large variation in design and outcome measures.

\section{Discussion}

Nineteen publications on the influence of serotonergic modulation on both gastrointestinal and psychiatric symptoms were identified ${ }^{24-42}$. Nine publications were excluded: seven studies did not meet the inclusion criteria $a^{36,37,39-42}$ and two sets of two duplicate studies were identified ${ }^{26,31,32,38}$. Of the remaining ten studies, that of Watson et al. reported on two different investigations ${ }^{27}$. Consequently, eleven were included in the qualitative analysis $24-31,33,34,43$. Of these, six studies scored 55 points or above $e^{24-28,43}$. These six studies were published more recently (median publication year, 1997) than the five studies which had a methodological quality score below 55 points (median publication year, 1984). Only one of the six studies that received an adequate methodological score evaluated the effect of an antidepressant agent ${ }^{26}$. The remaining five studies used $5-\mathrm{HT}_{3}$ antagonists and $5-\mathrm{HT}_{4}$ agonists ${ }^{24,25,27,28,43}$. Baseline gastroenterological and psychiatric symptomatology was present in all six studies. An association between the change in gastroenterological and psychiatric symptoms after intervention was present in five of the six studies. Gastroenterological symptoms improved in five studies. In four studies, both gastrointestinal and psychiatric symptoms improved during serotonergic treatment. In one study, no significant improvement in both gastroenterological and psychiatric symptoms was found. The wide variety of study designs, diagnostic criteria and outcome parameters hindered the pooling of data and made it difficult to draw firm general conclusions. However, these results are in line with a possible relationship between gastrointestinal and psychiatric symptoms, and hence support the concept of a 5-HT- driven 'brain-gut axis'. The ENS contains $95 \%$ of the body's $5-\mathrm{HT}$, and the remaining $5 \%$ is located in the $\mathrm{CNS}^{45}$. In addition to influencing serotonergic activity in the CNS, 
antidepressants down-regulate peripheral nociceptive pathways, which may account for some of their benefits ${ }^{46}$. Although it generally assumed that $5-\mathrm{HT}_{3}$ and $5-\mathrm{HT}_{4}$ (ant)agonists are predominantly pharmacologically active in the gastrointestinal tract, our results showed that both primarily centrally and peripherally active 5-HT modulators influenced psychiatric symptomatology in this specific patient group. Because the 'brain-gut axis' consists of an integrated network of pathways, pharmacological modulation at one level consequently modifies efferent and afferent activity at other levels of the 'braingut axis'.

Jackson et al. performed a meta-analysis on the use of antidepressants in the treatment of patients with functional gastrointestinal disorders, and concluded that they appeared to be effective ${ }^{17}$. Whether this improvement was independent of an effect of treatment on depression remained unresolved. Jailwala et al. evaluated the efficacy of pharmacological agents in IBS, and suggested a possible benefit of antidepressants (which were most often tricyclic antidepressants) in IBS patients ${ }^{18}$. However, it was still unclear whether antidepressants improved specific symptoms or had a more global effect.

Guidelines regarding the design of functional gastrointestinal studies have been well described ${ }^{47}$. In addition to these guidelines, we recommend that patients should be subcategorised into the diarrhoea-, constipation- and alternating- predominant types of IBS in trials assessing the influence of serotonergic manipulation in IBS, as $5-\mathrm{HT}$ is involved in both gastrointestinal physiology and affective disorders, and classification into diarrhoea- and constipation-predominant categories appears to reveal physiological differences between these types ${ }^{48-50}$. Our study shows an association between gastrointestinal and psychiatric symptoms; however, it is unclear whether a causal relationship exists. Although psychiatric measurements at baseline are an option in the guidelines ${ }^{47}$, in our opinion, in order to improve our understanding of the pathology in IBS and the role of the 'brain-gut axis', future studies should include both gastroenterological and psychiatric symptomatology at baseline and follow-up. As psychiatric symptomatology may be present in patients with IBS, the presence of possible psychiatric symptomatology or diagnosis should be assessed. In order to describe possible mood, anxiety or somatoform disorders, a structured psychiatric diagnostic interview should be performed. In addition, the level of psychiatric symptoms should be assessed (e.g. Hospital Anxiety and Depression Scale ${ }^{51}$ or Hamilton Depression Rating Scale ${ }^{52}$ ) at baseline and during follow-up.

It should be noted that most IBS studies are carried out in a tertiary-care clinics where psychiatric comorbidity is more often present than in IBS patients in a primary care setting or in those who never present to their doctor ${ }^{5,9}$. The relationship found in this study between gastrointestinal and psychiatric symptoms concerns investigations mostly conducted in tertiary referral centres. 
$60 \mid$ Chapier 3

In accordance with the recommendations of others ${ }^{47}$, it is important to discriminate between primary and secondary gastroenterological outcome parameters beforehand, and to perform an intention-to-treat analysis.

Furthermore, it is recommended that standardized global outcome parameters and standardised side-effect questionnaires are used, and that an adequate description of the presence of comorbidity and the use of co-medication is given.

The main conclusion to be drawn from this review is that, in IBS in the gastroenterological setting, a combination of both gastrointestinal and psychiatric pathology is present. Several types of serotonergic modulation are associated with improvement of gastrointestinal as well as psychiatric symptoms. Both primary peripheral and central $5-\mathrm{HT}$ manipulations were involved in these studies. Adjusted guidelines for combined gastrointestinal and psychiatric assessments are recommended in order to further elucidate the serotonergic interaction between gastrointestinal and psychiatric symptoms. 


\section{Appendix 3A}

Methodological criteria (total scoring). Each criterion must be applied independently of the other criteria.

\begin{tabular}{|c|c|c|}
\hline Criterion & Points & $\begin{array}{l}\text { Number of } \\
\text { studies } \\
\text { fulfilling } \\
\text { criterion }\end{array}$ \\
\hline \multicolumn{3}{|l|}{ A Selection and restriction ( 25 points) } \\
\hline 1 description of IBS: Rome criteria/Manning Criteria & 5 & 5 \\
\hline or other & $0-5$ & 2 \\
\hline 2 subdivision of IBS (diarrhoea/constipation) & 3 & 8 \\
\hline 3 description of level of gastrointestinal symptoms (scores) & 5 & 8 \\
\hline 4 description of psychiatric diagnosis: SCID/CIDI/MINI & 5 & 0 \\
\hline or other & 0.5 & 0 \\
\hline $\begin{array}{l}5 \text { description of level of psychiatric symptoms: } \\
\text { HDRS/HADS/GHQ/ZungNAS }\end{array}$ & 5 & 9 \\
\hline or other: & $0-5$ & 2 \\
\hline 6 description of other comorbidity and co-medication & 2 & $\overline{4}$ \\
\hline \multicolumn{3}{|l|}{ B Treatment allocation (4 points) } \\
\hline 1 allocation procedure (randomisation) blinded & 4 & 11 \\
\hline \multicolumn{3}{|l|}{ C Study size (6 points) } \\
\hline 1 study size based on power analysis & 3 & 3 \\
\hline 2 smallest group larger than 8 subjects & 1 & 11 \\
\hline 3 smallest group larger than or equal to 15 subjects & 1 & 11 \\
\hline 4 smallest group larger than 30 subjects & 1 & 7 \\
\hline \multicolumn{3}{|l|}{ D Prognostic comparability (6 points) } \\
\hline 1 duration of the gastroenterological symptoms & 1 & 5 \\
\hline 2 duration of the psychiatric symptoms & 1 & 1 \\
\hline 3 baseline scores for gastroenterological outcome measures & 1 & 9 \\
\hline 4 baseline scores for psychiatric outcome measures & 1 & 8 \\
\hline 5 comparable demographics (age/gender) & 1 & 11 \\
\hline 6 comparable with regard to subtyping (IBS/psychopathology) & 1 & 7 \\
\hline \multicolumn{3}{|l|}{ E Drop-outs/follow-up (4 points) } \\
\hline 1 number of drop-outs given in each group if number of drop-outs given: & 2 & 9 \\
\hline 2 reasons for withdrawal given in each group or no drop-outs & 1 & 8 \\
\hline 3 drop-outs not leading to bias (or less than $10 \%$ ) & 1 & 3 \\
\hline \multicolumn{3}{|l|}{ F Intervention $=$ experimental $(6$ points $)$} \\
\hline $\begin{array}{l}1 \text { description of dose regimen (times per day, quantity) duration of each } \\
\text { treatment period and wash-out period if applicable more than } 4 \text { weeks }\end{array}$ & 1 & 11 \\
\hline 2 more than 6. weeks & 2 & 8 \\
\hline 3 more than 8 weeks & 1 & 6 \\
\hline 4 compliance presented (pill count or blood samples) & 2 & 1 \\
\hline \multicolumn{3}{|l|}{$G$ Intervention = placebo or other control (6 points) } \\
\hline $\begin{array}{l}1 \text { description of dose regimen (times per day, quantity) duration of each } \\
\text { treatment period and wash-out period if applicable more than } 4 \text { weeks }\end{array}$ & 1 & 11 \\
\hline 2 more than 6 weeks & 2 & 8 \\
\hline 3 more than 8 weeks & 1 & 6 \\
\hline 4 compliance presented (pill count or blood samples) & 2 & 1 \\
\hline \multicolumn{3}{|l|}{ H Outcome measures (20 points) } \\
\hline 1 use of global outcome parameters & 3 & 9 \\
\hline 2 use of standardized global outcome parameters & 3 & 5 \\
\hline 3 primary (core) gastroenterological outcome measures & 3 & 3 \\
\hline 4 secondary gastroenterological outcome measures & 3 & 11 \\
\hline 5 psychiatric assessments: HDRS/HADS/GHQ/Zung/VAS & 5 & 9 \\
\hline or other & $0-5$ & 2 \\
\hline 6 blinded assessment of measurements & 3 & 11 \\
\hline
\end{tabular}




\begin{tabular}{lcc}
\hline Follow-up period (4 points) & 2 & 2 \\
1 follow-up period & 1 & 2 \\
2 timing comparable & 1 & 1 \\
3 measurements three months or longer following acute treatment & 2 & 6 \\
$J$ Description of side-effects in each group (9 points) & 2 & 0 \\
1 self report (e.g. global questioning) & 2 & 0 \\
2 specific systematic questionnaires & 2 & 2 \\
3 specification (listing) of systematic questionnaires & 1 & 6 \\
4 objective measurements (e.g. blood pressure, pulse rate, weight) & & \\
5 blinded assessment of side-effects measurements & 2 & 10 \\
Analyses and presentation of data (10 points) & 2 & 11 \\
1 data presented on results of general outcome measures & 2 & 11 \\
2 data presented on results of gastroenterological outcome measures & 2 & 3 \\
3 data presented on results of psychiatric symptoms & 2 & 10 \\
4 intention-to-treat analyses has been done & 100 & \\
5 adequate statistical methods have been used & \\
\hline
\end{tabular}

GHQ, General Health Questionnaire; HADS, Hospital Anxiety and Depression Scale; HDRS. Hamilton Depression Rating Scale; IBS, irritable bowel syndrome; IBSQOL, Irritable Bowel Syndrome Quality of Life Questionnaire; MINI, Mini International Neuropsychiatric Interview; 5-HT, serotonin; SCID, Structured Clinical Interview for DSM-IV; SSRI, selective serotonin reuptake inhibitor; TCA, tricyclic antidepressant; VAS, visual analogue scale; Zung, Zung Depression Rating Scale. 
Revigw: Serotonergic modulation in irritable bowel syndrome $\mid 69$

\section{References}

1. Mayer EA. Emerging disease model for functional gastrointestinal disorders. Am J Med 1999; 107:12S-19S.

2. Talley NJ. Serotonergic neuroenteric modulators. Lancet 2001; 358: 2061-2068.

3. Mayer EA, Gebhart GF. Basic and clinical aspects of visceral hyperalgesia. Gastroenterology 1994;107:271-293.

4. Noyes R, Jr., Cook B, Garvey $M$, Summers R. Reduction of gastrointestinal symptoms following treatment for panic disorder. Psychosomatics 1990;31:75-79.

5. Walker EA, Katon WJ, Jemelka RP, Roy-Bryne PP. Comorbidity of gastrointestinal complaints, depression, and anxiety in the Epidemiologic Catchment Area (ECA) Study. Am J Med 1992;92:26S-30S.

6. Lydiard RB, Fossey MD, Marsh W, Ballenger JC. Prevalence of psychiatric disorders in patients with irritable bowel syndrome. Psychosomatics 1993;34:229-234.

7. Lydiard RB, Greenwald S, Weissman MM, Johnson J, Drossman DA, Ballenger JC. Panic disorder and gastrointestinal symptoms: findings from the NIMH Epidemiologic Catchment Area project. Am J Psychiatry 1994;151:64-70.

8. Zaubler TS, Katon W. Panic disorder and medical comorbidity: a review of the medical and psychiatric literature. Bull Menninger Clin 1996;60:A12-38.

9. Lydiard RB. Anxiety and the irritable bowel syndrome: psychiatric, medical, or both? J Clin Psychiatry 1997;58:51-8; discussion 59-61.

10. Ford MJ, Miller PM, Eastwood J, Eastwood MA. Life events, psychiatric illness and the irritable bowel syndrome. Gut 1987;28:160-165.

11. Walker EA, Roy-Byrne PP, Katon WJ. Irritable bowel syndrome and psychiatric illness. Am J Psychiatry 1990;147:565-572.

12. Walker EA, Roy-Byrne PP, Katon WJ, Li L, Amos D, Jiranek G. Psychiatric illness and irritable bowel syndrome: a comparison with inflammatory bowel disease. Am J Psychiatry 1990;147:1656-1661.

13. Fock KM, Chew CN, Tay LK, Peh LH, Chan S, Pang EP. Psychiatric illness, personality traits and the irritable bowel syndrome. Ann Acad Med Singapore 2001;30:611-614.

14. Hyams JS, Burke G, Davis PM, Rzepski B, Andrulonis PA. Abdominal pain and irritable bowel syndrome in adolescents: a community- based study. J Pediatr 1996;129:220-226.

15. Gorard DA, Libby GW, Farthing MJ. Influence of antidepressants on whole gut and orocaecal transit times in health and irritable bowel syndrome. Aliment Pharmacol Ther 1994;8:159-166.

16. O'Malley PG, Jackson JL, Santoro J, Tomkins G, Balden $E$, Kroenke K. Antidepressant therapy for unexplained symptoms and symptom syndromes. J Fam Pract 1999;48:980-990.

17. Jackson JL, O'Malley PG, Tomkins G, Balden E, Santoro J, Kroenke K. Treatment of functional gastrointestinal disorders with antidepressant medications: a meta-analysis. Am J Med 2000;108:65-72.

18. Jailwala J, Imperiale TF, Kroenke K. Pharmacologic treatment of the irritable bowel syndrome: a systematic review of randomized, controlled trials. Ann Intern Med 2000;133: 136-147.

19. Fishbain D. Evidence-based data on pain relief with antidepressants. Ann Med 2000; 32: 305-316.

20. Akehurst $R$, Kaltenthaler $E$. Treatment of irritable bowel syndrome: a review of randomised controlled trials. Gut 2001;48:272-282.

21. de Vet HCW, de Bie RA, van der Heijden GJMG, Verhagen AP, Sijpkes P, Knipschild PG. Systematic reviews on the basis of methodolodical criteria. Physiotherapy 1997;83:284-289.

22. Menting JE, Honig $A$, Verhey FR, Hartmans $M$, Rozendaal $N$, de Vet $H C$, van Praag HM. Selective serotonin reuptake inhibitors (SSRIs) in the treatment of elderly depressed patients: a qualitative analysis of the literature on their efficacy and side-effects. Int Clin Psychopharmacol 1996;11:165-175. 
$70 \mid$ Chapter 3

23. Klaassen $T$, Verhey FRJ, Sneijders GHJM, Rozendaal N, de Vet HCW, van Praag HM. Treatment of Depression in Parkinson's Disease: A meta-Analysis. J Neuropsychiatry Clin Neurosci 1995;7:281-286.

24. Schutze K, Brandstatter G, Dragosics B, Judmaier G, Hentschel E. Double-blind study of the effect of cisapride on constipation and abdominal discomfort as components of the irritable bowel syndrome. Aliment Pharmacol Ther 1997;11:387-394.

25. Van Outryve M, Milo R, Toussaint J, Van Eeghem P. "Prokinetic" treatment of constipationpredominant irritable bowel syndrome: a placebo-controlled study of cisapride. J Clin Gastroenterol 1991;13:49-57.

26. Greenbaum DS, Mayle JE, Vanegeren LE, Jerome JA, Mayor JW, Greenbaum RB, Matson RW, Stein GE, Dean HA, Halvorsen NA, et al. Effects of desipramine on irritable bowel syndrome compared with atropine and placebo. Dig Dis Sci 1987;32:257-266.

27. Watson ME, Lacey L, Kong S, Northcutt AR, McSorley D, Hahn B, Mangel AW. Alosetron improves quality of life in women with diarrhea-predominant irritable bowel syndrome. Am J Gastroenterol 2001;96:455-459.

28. Maxton DG, Morris J, Whorwell PJ. Selective 5-hydroxytryptamine antagonism: a role in irritable bowel syndrome and functional dyspepsia? Aliment Pharmacol Ther 1996;10: 595-599.

29. Vij JC, Jiloha RC, Kumar N, Madhu SV, Malika V, Anand BS. Effect of antidepressant drug (doxepin) on irritable bowel syndrome patients. Indian J Psychiat 1991;33:243-246.

30. Bergmann $M$, Heddergott $A$, Schlosser $T$. Die therapie des colons irritable mit trimipramin (Herphonal) - Eine kontrolierte studie. Z klin Med 1991;46:1621-1628.

31. Myren J, Lovland B, Larssen SE, Larsen S. A double-blind study of the effect of trimipramine in patients with the irritable bowel syndrome. Scand J Gastroenterol 1984;19:835-843.

32. Myren J, Lovland B, Larssen SE, Larsen S. Psychopharmacologic drugs in the treatment of the irritable bowel syndrome. A double blind study of the effect of trimipramine. Ann Gastroenterol Hepatol (Paris) 1984;20:117-123.

33. Myren J, Groth H, Larssen SE, Larsen S. The effect of trimipramine in patients with the irritable bowel syndrome. A double-blind study. Scand J Gastroenterol 1982;17:871-875.

34. Heefner JD, Wilder RM, Wilson ID. Irritable colon and depression. Psychosomatics 1978;19: 540-547.

35. Lancaster-Smith MJ, Prout BJ, Pinto T, Anderson JA, Schiff AA. Influence of drug treatment on the irritable bowel syndrome and its interaction with psychoneurotic morbidity. Acta Psychiatr Scand 1982;66:33-41.

36. Alevizos B, Christodoulou GN, loannidis C, Voulgari A, Mantidis A, Spiliadis C. The efficacy of amineptine in the treatment of depressive patients with irritable bowel syndrome. Clin Neuropharmacol 1989;12:S66-76.

37. Steinhart MJ, Wong PY, Zarr ML. Therapeutic usefulness of amitriptyline in spastic colon syndrome. Int J Psychiatry Med 1981;11:45-57.

38. Greenbaum DS. Preliminary report on antidepressant treatment of irritable bowel syndrome: comments on comparison with anxiolytic therapy. Psychopharmacol Bull 1984;20:622-628.

39. Tripathi BM, Misra NP, Gupta AK. Evaluation of tricyclic compound (trimipramine) vis-a-vis placebo in irritable bowel syndrome. J Assoc Physicians India 1983;31:201-203.

40. Tanum L, Malt UF. A new pharmacologic treatment of functional gastrointestinal disorder. A double-blind placebo-controlled study with mianserin. Scand J Gastroenterol 1996;31: 318-325.

41. Rajagopalan $M$, Kurian $G$, John J. Symptom relief with amitriptyline in the irritable bowel syndrome. J Gastroenterol Hepatol 1998;13:738-741.

42. Tanum L, Malt UF. Personality traits predict treatment outcome with an antidepressant in patients with functional gastrointestinal disorder. Scand J Gastroenterol 2000;35:935-941.

43. Camilleri M, Northcutt AR, Kong S, Dukes GE, MCSorley D, Mangel AW. Efficacy and safety of alosetron in women with irritable bowel syndrome: a randomised, placebo-controlled trial. Lancet 2000;355:1035-1040. 
44. Hahn BA, Kirchdoerfer LJ, Fullerton S, Mayer E. Evaluation of a new quality of life questionnaire for patients with irritable bowel syndrome. Aliment Pharmacol Ther 1997;11: 547-552.

45. Gershon MD. Review article: roles played by 5-hydroxytryptamine in the physiology of the bowel. Aliment Pharmacol Ther 1999;13 Suppl 2:15-30.

46. Gebhart GF. Visceral nociception: consequences, modulation and the future. Eur J Anaesthesiol Suppl 1995;10:24-27.

47. Veldhuyzen van Zanten SJ, Talley NJ, Bytzer $P$, Klein KB, Whorwell PJ, Zinsmeister AR. Design of treatment trials for the functional gastrointestinal disorders. In: Drossman DA, Corazziari E, Talley NJ, Thompson WG, Whitehead WE, editors. Rome II: the functional gastrointestinal disorders. 2 ed. McLean: Degnon Associates; 2000:577-622.

48. Prior A, Maxton DG, Whorwell PJ. Anorectal manometry in irritable bowel syndrome: differences between diarrhoea and constipation predominant subjects. Gut 1990;31:458-462.

49. Aggarwal A, Cutts TF, Abell TL, Cardoso S, Familoni B, Bremer J, Karas J. Predominant symptoms in irritable bowel syndrome correlate with specific autonomic nervous system abnormalities. Gastroenterology 1994;106:945-950.

50. Elsenbruch S, Orr WC. Diarrhea- and constipation-predominant IBS patients differ in postprandial autonomic and cortisol responses. Am J Gastroenterol 2001;96:460-466.

51. Zigmond AS, Snaith RP. The hospital anxiety and depression scale. Acta Psychiatr Scand 1983:67:361-370.

52. Hamilton M. Development of a rating scale for primary depressive illness. $\mathrm{Br} \mathrm{J}$ Soc Clin Psychol 1967;6:278-296. 


\section{Chapter 4}

Fatty acid profile and affective dysregulation in irritable bowel syndrome

TO Kilkens, A Honig, M Maes, R Lousberg, RJ Brummer

Lipids 2004; 39: 425-431 
74 Chapter 4

\begin{abstract}
Background

Irritable bowel syndrome (IBS) is a functional gastrointestinal disorder with a high co-occurrence of affective dysregulation. Affective disorders have been associated with specific changes in the polyunsaturated fatty acids (PUFAs) and cholesterol profile. In IBS, similar changes may be present as have been reported in patients with affective disorders.

Aim

This exploratory study investigates (i) the level of affective dysregulation in IBS patients and healthy controls; (ii) PUFAs and cholesterol profiles in IBS patients compared with controls; and (iii) associations between PUFAs and cholesterol parameters with the level of affective dysregulation.
\end{abstract}

Methods

Blood samples were obtained for determination of the FA composition of plasma phospholipids and serum cholesterol in 23 diarrhoea-predominant IBS patients and 23 healthy matched controls. Affective dysregulation was scored using the Symptom Check List depression scale, the Hospital Anxiety and Depression Scale and the Hamilton Depression Rating Scale.

Results

The level of affective dysregulation was higher in IBS compared with controls. PUFAs and cholesterol profile did not differ significantly between groups. Total n-3 PUFAs and cholesterol were significantly negatively and the ratio of $n-6$ to $n-3$ PUFAs and the ratio of arachidonic acid to eicosapentaenoic acid were significantly positively associated with the level of affective dysregulation.

\title{
Conclusions
}

The findings of the present study reveal that affective dysregulation was higher in IBS patients compared with healthy controls and that changes in PUFAs and cholesterol profiles were significantly associated with level of affective dysregulation. These results warrant further studies regarding the role of PUFAs and cholesterol status in the co-occurrence of affective dysregulation and functional gastrointestinal disorders. 


\section{Introduction}

Irritable bowel syndrome (IBS) is a functional gastrointestinal disorder characterized by abdominal pain or discomfort associated with alteration in defecation, in the absence of structural or biochemical abnormalities that can be identified utilizing currently available tests ${ }^{1}$. About $10-20 \%$ of the Western population has symptoms consistent with a diagnosis of $\mathrm{IBS}^{2}$. The quality of life of individuals with IBS is lower than that of individuals with congestive heart failure; health care and employer costs of IBS are high ${ }^{3,4}$. IBS can be classified into diarrhoea-predominant (d-IBS), constipation-predominant, and mixed type of IBS. The pathophysiology of IBS is poorly understood. A multicomponent conceptual model of IBS has been postulated, involving physiological, affective, cognitive, and behavioural factors ${ }^{5}$. Within this model the 'brain-gut axis' has gained interest. The brain-gut axis is a theoretical model describing the bidirectional neural pathways linking cognitive and emotional centres in the brain to neuroendocrine centres, the enteric nervous system, and the immune system. This brain-gut axis plays a major role in the concept of IBS ${ }^{6}$.

The prevalence of affective dysregulation in IBS patients seen in a gastroenterological setting is estimated between 40 and $90 \%{ }^{7-10}$. Chronic modulation of serotonergic activity has been widely applied in the treatment of both affective disorders and IBS. Treatment using serotonergic modulators has been reported to influence both gastrointestinal and psychiatric symptoms in $\mathrm{IBS}^{11}$. Disturbed serotonergic metabolism seems especially prevalent in the d-IBS type of $1 B S^{12-14}$. The high rates of affective dysregulation in IBS patients may be a specific and integral part of IBS, rather than a non-specific comorbid syndrome related to a chronic intestinal disease ${ }^{11,15,16}$.

In patients with affective disorders, such as major depression, specific changes in the composition of PUFAs and cholesterol profiles have been observed. Specifically, affective disorders are associated with diminished levels of plasma total $n-3$ PUFAs, a higher ratio of $n-6$ to $n-3$ PUFAs, a higher ratio of arachidonic acid (AA) to eicosapentaenoic acid (EPA), and lower serum total cholesterol levels ${ }^{17-21}$.

The role of PUFAs and cholesterol in the pathophysiology of affective disorders has been related to alterations in central nervous system (CNS) serotonergic neurotransmitter systems, membrane-bound receptor function, and enzymatic activity caused by disruption of neuronal membrane composition and structure ${ }^{22-24}$. In addition, the immune system may play a role in the mechanism of action of PUFAs in affective disorders ${ }^{25-26}$. 
$76 \mid$ Chapter 4

Consequently, more knowledge of PUFAs composition in d-IBS patients may have both pathophysiological and therapeutic implications. As IBS is associated with a high co-occurrence of affective dysregulation, and as affective disorders have been associated with specific changes in the PUFAs and cholesterol profile, we hypothesized that in d-IBS similar changes may be present as have been reported in patients with affective disorders. This exploratory study investigates: (i) the level of affective dysregulation in d-IBS patients and healthy controls; (ii) PUFAs and cholesterol profiles in d-IBS patients compared with controls; and (iii) associations between PUFAs and cholesterol parameters, respectively, and the level of affective dysregulation.

\section{Materials and methods}

\section{Subjects}

Patients were recruited from the outpatient Department of Gastroenterology of University Hospital Maastricht (Maastricht, The Netherlands). Healthy control subjects were enrolled via advertising in local papers. The Medical Ethics Committee of the University Hospital Maastricht approved the study protocol, and all subjects gave their written informed consent before the start of the study. The required number of subjects was based on results of other ongoing studies at our department and an estimated effect size of 0.9 (based on the standardized mean difference between postmyocardial infarction patients with co-morbid depression and postmyocardial infarction patients without co-morbid depression with respect to the AA/EPA ratio), with $\beta=0.2$ and $\alpha=0.05$ resulting in $n=21$ per group. Patients fulfilled the diagnosis of $d-I B S$ according to the ROME II criteria, as diagnosed by an experienced gastroenterologist ${ }^{27}$. These diagnostic criteria for IBS are abdominal discomfort or pain for at least 12 weeks, which need not to be consecutive, in the preceding 12 months that has two out of three features: (i) relieved with defecation; and/or (ii) onset associated with a change in frequency of stool; and/or (iii) onset associated with a change in form of stool in absence of structural or metabolic abnormalities to explain symptoms. Additional criteria for $d-$ IBS are more than three bowel movements a day or loose/watery stools or urgency. Before participating in the study, all subjects were screened by a standardized psychiatric examination comprising the Mini International Neuropsychiatric Interview (MINI) to determine the present psychiatric state ${ }^{28}$. The level of affective dysregulation was assessed by using the depression scale of the Symptom Check List (SCL-depression) ${ }^{29}$, the Hospital Anxiety and Depression Scale $(\mathrm{HADS})^{30}$, and the 17-item Hamilton Depression Rating Scale $\left(\right.$ HAM-D17) ${ }^{31}$. Physical health was assessed by means of a standard physical 
examination and urine test. A pregnancy test was performed on all female subjects. Exclusion criteria were abdominal surgery, use of medications other than oral contraceptives within 14 days prior to testing, lactose malabsorption (using $\mathrm{H}_{2}$-breath test) ${ }^{32,33}$, a positive first-degree psychiatric family history ${ }^{34}$, any history of psychiatric disease or use of psychoactive medication, premenstrual syndrome, dieting, pregnancy or lactation, excessive alcohol intake ( $>20$ alcoholic consumptions a week), and hypertension (diastolic $>100$ $\mathrm{mmHg}$, systolic $>170 \mathrm{mmHg}$ ), respectively. For the control subjects, the same exclusion criteria applied as those for the patients. Additional exclusion criteria for the control subjects were current or history of gastrointestinal disorder, current psychiatric or psychological symptomatology defined as: a diagnosis on the MINI, SCL-depression score for females $\geq 28$ and for males $\geq 23$, HADS scores $\geq 8$, and HAM-D17 scores above 18 .

\section{Study design}

To eliminate possible bias, all women were evaluated in the follicular phase of the menstrual cycle or while taking oral contraception. To exclude possible seasonal variation, the patient and corresponding matched control subject were evaluated within three months time. They were asked to abstain from heavy physical exercise and consumption of alcoholic beverages the day before their visit. Subjects attended the laboratory after an overnight fast (after 10:00 PM, no eating, drinking, or smoking allowed) at 8:00 AM for blood collection.

\section{Biochemical parameters}

Blood for plasma PUFAs determination was sampled in Vacutainer (K2E) tubes and was immediately placed on ice. Blood for serum cholesterol was sampled in Vacutainer (SST) tubes. All samples were centrifuged within 30 minutes (ten minutes, $900 \mathrm{~g}, 4^{\circ} \mathrm{C}$ ) and stored at $-80^{\circ} \mathrm{C}$ until analysis. PUFAs samples were preserved from oxidation by blanketing them with nitrogen ${ }^{35}$. Samples were analysed blind as to subject status in a single run. Plasma phospholipid concentrations were determined, after isolation from plasma, as fatty acid methyl esters (FAME). To $100 \mu$ of plasma, $300 \mu \mathrm{l}$ of $1 \%$ EDTA and $50 \mu \mathrm{l}$ of internal standard was added $(620 \mathrm{mg} / \mathrm{l}$ 1,2-dinonadecanoyl-sn-glycero-3phosphocholine (Avanti Polar, Alabaster, AL); to all solutions butylated hydroxytoluene (BHT) was added as antioxidant. Total lipids were extracted from plasma with a modified extraction according to Folch et al. ${ }^{36}$. The phospholipid fraction was isolated by multiple solid-phase extraction (Bond Elut aminopropyl bonded silica columns $3 \mathrm{ml}, 500 \mathrm{mg}$; Varian, Palo Alto, CA) as described previously ${ }^{37}$. Phospholipids were hydrolysed, and the resulting FA converted to methyl esters with boron trifluoride/methanol (14\%). The FAME's were determined by a gas chromatograph with flame ionisation detection 
(HP5890 series II; Hewlett-Packard, Palo Alto, CA) with dual capillary column technique for optimal separation of cis- and trans-FAME (BP1 $50 \mathrm{~m} \times 0.22 \mathrm{~mm}$, i.d. $0.1 \mu \mathrm{m}, \mathrm{BPX} 7050 \mathrm{~m} \times 0.22 \mathrm{~mm}$, i.d. $0.25 \mu \mathrm{m}$; SGE, Melbourne, Australia). Serum total cholesterol, high-density lipoprotein cholesterol (HDL-C) and lowdensity lipoprotein cholesterol (LDL-C) were measured by a commercially available colorimetric assay (Beckman Synchron LX20 systems, Fullerton, CA).

Statistical analysis

Group differences (IBS vs. control) in demographic data were analysed using independent $t$ tests. PUFAs profile was the primary outcome parameter consisting of three FA outcome parameters (total n-3 PUFAs, ratio of n-6 to n-3 PUFAs, and ratio of AA to EPA). The secondary outcome parameter was cholesterol profile (serum total cholesterol, HDL-C, and LDL-C). Group differences in outcome parameters were assessed by means of two multivariate ANOVA (one for FA parameters, one for cholesterol parameters). Associations between outcome parameters with level of affective dysregulation as indicated by SCL-depression, HADS, and HAM-D17 scores, respectively, were analysed by linear regression. Cook's distance was used to identify possible influential cases according to the lines described by Hair ${ }^{38}$. Data are presented as mean (SE). Two-tailed p-values $\leq 0.05$ were considered statistically significant. Statistical analyses were performed using the SPSS 10.0 for Windows software package (Chicago, IL).

\section{Results}

\section{Sample description}

Table 4.1 summarizes characteristics of the 46 participants ( 23 patients and 23 healthy controls). No significant differences were found between the two groups with respect to sex, age, body mass index (BMI), contraceptive use, or alcohol and cigarette consumption ( $p$-values $\geq 0.3$ ). Severity of affective dysregulation, as indicated by the SCL-depression, HADS, and HAM-D17 scores, was significantly higher in IBS patients than in healthy controls ( $p$-values $\leq 0.02$ ). 
Table 4.1 Demographic data of patients with irritable bowel syndrome (IBS) and healthy matched controls (mean (SE)).

\begin{tabular}{lccc}
\hline & $\begin{array}{c}\text { IBS } \\
(n=23)\end{array}$ & $\begin{array}{c}\text { Controls } \\
(n=23)\end{array}$ & $\begin{array}{c}\text { Patients vs. Controls } \\
\text { p value }\end{array}$ \\
\hline Females $(\mathrm{n})$ & $14 / 23$ & $14 / 23$ & \\
Oral contraceptives $(\mathrm{n})$ & $11 / 14$ & $11 / 14$ & \\
Age & $32.9(2.3)$ & $28.6(3.3)$ & 0.3 \\
Body mass index & $23.1(0.9)$ & $22.7(0.5)$ & 0.7 \\
Alcohol units/d & $1.12(0.6)$ & $0.53(0.1)$ & 0.4 \\
Cigarettes/d & $2.10(0.8)$ & $1.27(0.7)$ & 0.4 \\
Diagnosis on MINI & $7^{\mathrm{b}}$ & 0 & - \\
SCL-depression & $20.5(1.2)$ & $17.3(0.5)$ & 0.02 \\
HADS & $6.22(0.7)$ & $3.17(0.6)$ & 0.002 \\
HAM-D17 & $4.2(0.8)$ & $0.83(0.2)$ & $<0.001$ \\
\hline
\end{tabular}

MINI, Mini International Neuropsychiatric Interview; HAM-D17, 17-item Hamilton Depression Rating Scale; SCL, Symptom Check List; HADS, Hospital Anxiety and Depression Scale.

${ }^{a}$ Independent samples $t$-test comparing IBS patients vs. control subjects; ${ }^{b}$ Seven subjects had a psychiatric diagnosis: depression $(n=2)$, agoraphobia $(n=3)$, social phobia $(n=2)$, and anxiety disorder $(n=2)$, respectively.

\section{FA profile of plasma phospholipids}

The amounts of the various FA were expressed as weight percentages (wt\%) of total FA as well as absolute concentrations (mg/l). In total, 35 FA were identified, but FA percentages $<0.1 \%$ were not recorded; therefore, 26 FA are listed in Table 4.2. The following FA combinations were calculated: $\Sigma$ SAFA (sum of all saturated FA), $\Sigma$ MUFA (sum of all monounsaturated FA), $\sum$ PUFAs (sum of all PUFAs), $\sum n-6$ PUFAs, $\sum n-3$ PUFAs, ratio of n-6 to n-3 PUFAs, and ratio of $A A$ to EPA $(20: 4 n-6 / 20: 5-n 3)$.

\section{PUFAs and cholesterol profile}

In Table 4.2 the FA outcome parameters and in Table 4.3 the cholesterol parameters are listed. As can be seen in these tables, there was no difference in outcome parameters between subject groups. Multivariate analyses did not show a significant difference in total n-3 PUFAs, ratio of n-6 to n-3 PUFAs, and ratio of AA to EPA $\left[F_{(\mathrm{HOT})}=0.4, \mathrm{df}=3.42, \mathrm{p}=0.8\right]$ nor in total cholesterol, $\mathrm{HDL}-\mathrm{C}$, and $L D L-C$ between IBS patients and controls $\left[F_{(H O T)}=1.8, d f=3.42, p=0.2\right]$, with no univariate significance. 
Chaptar $A$

Table 4.2 Descriptive (wt\% of total FA and absolute levels in $\mathrm{mg} / \mathrm{l}$ ) as well as outcome parameters of FA profile of plasma phospholipids in irritable bowel syndrome (IBS) patients and healthy controls (mean (SE)).

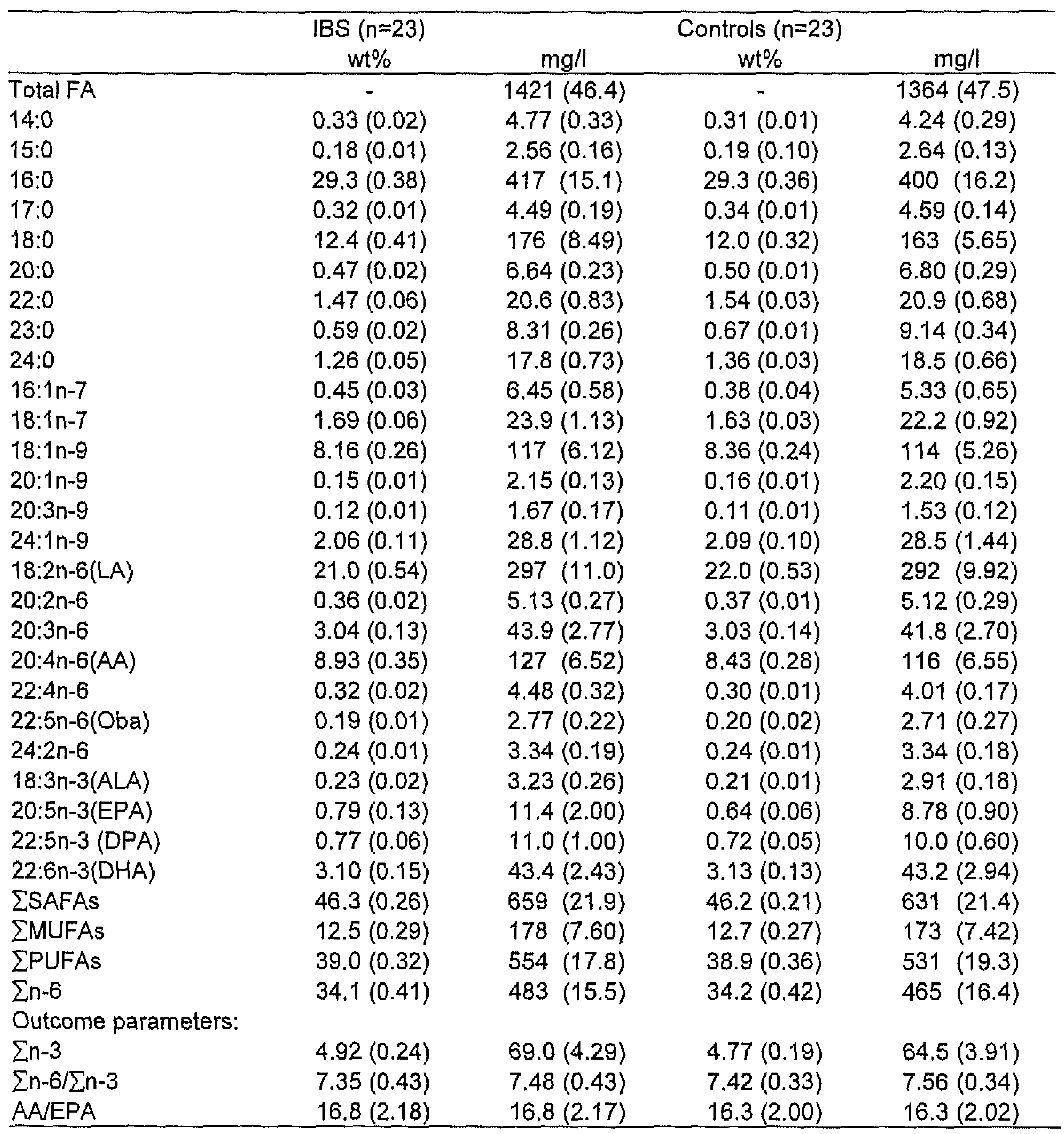

$A A$, arachidonic acid; $A L A$, a-linolenic acid; $D H A$, docosahexaenoic acid, DPA, docosapentaenoic acid; EPA, eicosapentaenoic acid; LA, linoleic acid; Oba, osbond acid; $\Sigma S A F A s:$ sum of all saturated fatty acids; ¿MUFAs: sum of all monounsaturated fatty acids; $\sum$ PUFAs: sum of all polyunsaturated fatty acids; $\sum n-6$ : sum of all n-6 PUFAs; $\sum n-3$ : sum of all n-3 PUFAs. 
Table 4.3 Serum total cholesterol, high-density lipoprotein cholesterol (HDL-C) and low-density lipoprotein cholesterol (LDL-C) in irritable bowel syndrome (IBS) patients and healthy controls (mean (SE)).

\begin{tabular}{lcc}
\hline & IBS $(\mathrm{n}=23)$ & Controls $(\mathrm{n}=23)$ \\
\hline Total cholesterol (mmol/l) & $5.13(0.18)$ & $5.09(0.19)$ \\
HDL-C (mmol/) & $1.09(0.07)$ & $1.22(0.08)$ \\
LDL-C (mmol/l) & $3.31(0.17)$ & $3.39(0.18)$ \\
\hline
\end{tabular}

Associations between PUFAs and total cholesterol with level of affective dysregulation

Total n-3 PUFAs tended to be significantly negatively associated with SCLdepression scores $\left[F=3.5, p=0.07, R^{2}=0.07\right]$. Cook's distance analyses showed that the regression model was unstable as indicated by 2 large Cook's measures; subsequently, these two cases (2 patients) were excluded. After removal of these two cases, total n-3 PUFAs was significantly negatively associated with SCL-depression scores $\left[F=8.6, p=0.006, R^{2}=0.18\right]$. Total $n-3$ PUFAs tended to be negatively associated with HADS scores $[F=3.5, p=0.06$, $\left.R^{2}=0.08\right]$. The ratio $n-6$ to $n-3$ PUFAs was significantly positively associated with SCL-depression scores $\left[F=11.9, p=0.002, R^{2}=0.22\right]$ and HADS scores $\left[F=4.8, p=0.03, R^{2}=0.11\right]$. The ratio of $A A$ to $E P A$ was significantly positively associated with $S C L$-depression scores $\left[F=12.9, p=0.002, R^{2}=0.24\right]$. Total serum cholesterol was significantly negatively associated with $\mathrm{SCL}$-depression scores $\left[F=7.6, p=0.005, R^{2}=0.15\right]$. PUFAs and cholesterol profiles were not significantly associated with Hamilton scores ( $p$-values $>0.2$ ). As an example, Figure 4.1 illustrates the associations between total n-3 PUFAs and ratio of AA to EPA with SCL-depression scores.

A

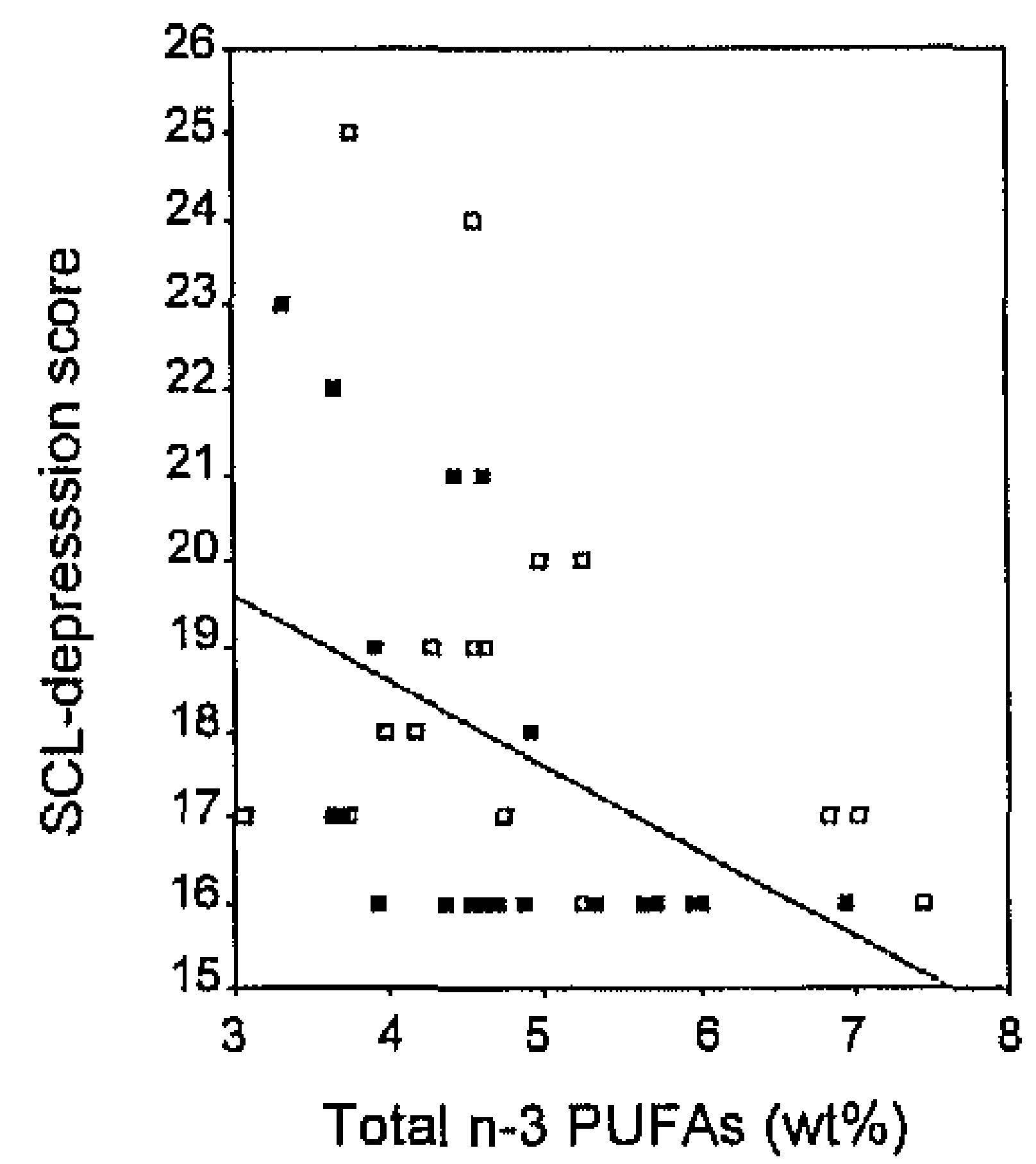

B

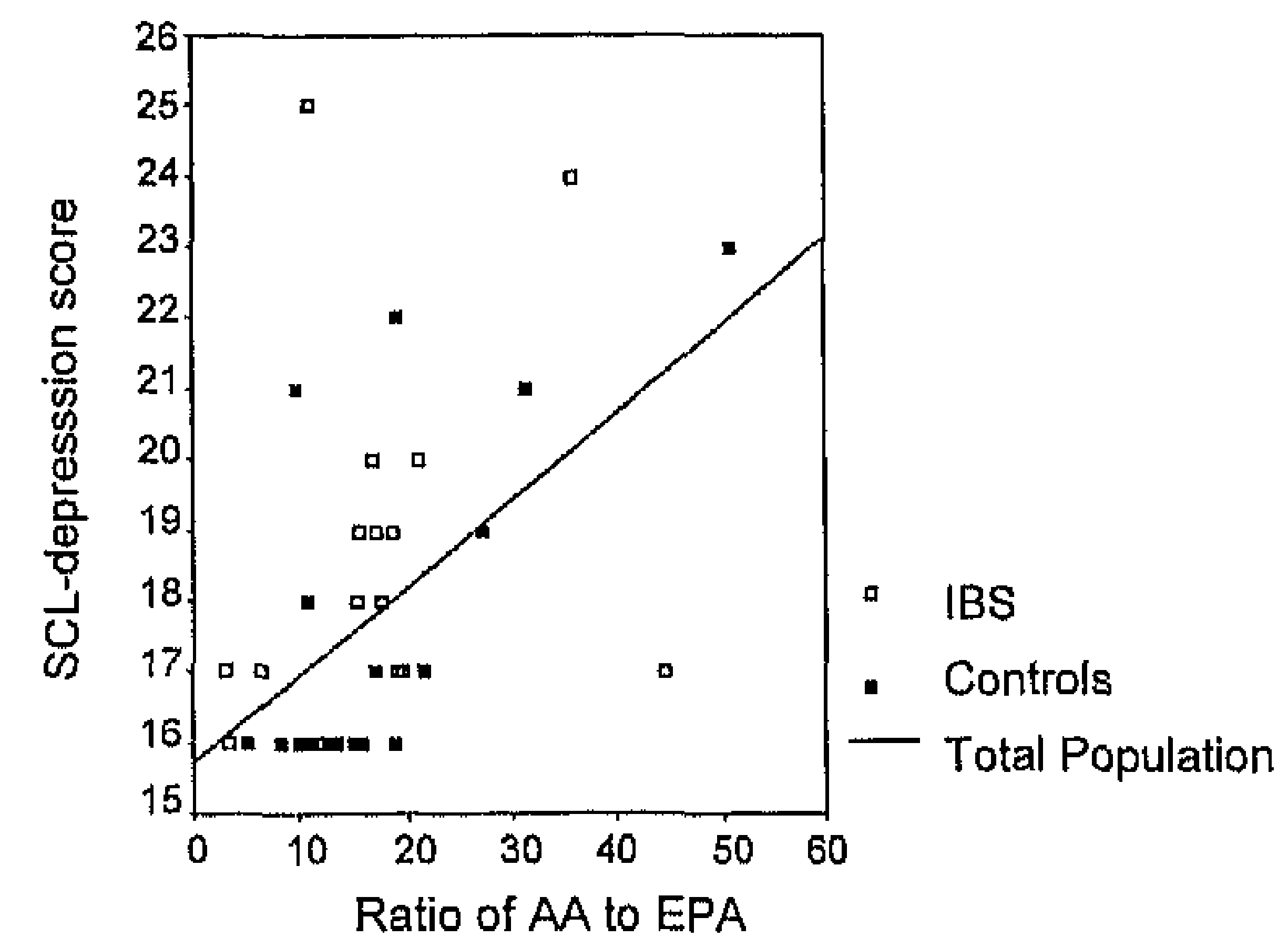

Figure 4.1 Associations between (A) total percentage n-3 PUFAs in phospholipids ( $w$ t $\%$ of total FA) and (B) ratio of arachidonic acid (AA) to eicosapentaenoic (EPA) with level of affective dysregulation as indicated by Symptom Check List (SCL)-depression score. IBS, irritable bowel syndrome. 


\section{Discussion}

The patient group demonstrated a higher level of affective dysregulation compared with the control group but did not differ in PUFAs and cholesterol profiles. However, total n-3 PUFAs and cholesterol were significantly negatively associated and the ratio of $n-6$ to $n-3$ PUFAs as well as the ratio of AA to EPA were significantly positively associated with level of affective dysregulation, respectively.

To our knowledge no previous studies have determined possible changes in PUFAs profile in IBS patients compared with healthy matched controls.

IBS patients had increased levels of affective dysregulation, and PUFAs and cholesterol profiles were significantly associated with affective dysregulation. However, we did not show differences in PUFAs and cholesterol indices between IBS patients and controls, as have been reported in patients with affective disorders. This may be explained by the fact that the level of affective dysregulation in our IBS patients was attenuated compared with that of patients primarily presenting with affective disorders. In addition, we chose to study the d-IBS type of IBS, because classification into subtypes appears to reflect physiological differences between each type ${ }^{39,40}$, and we excluded patients with a previous psychiatric diagnosis or first-degree family history of affective disorders ${ }^{41}$. As a result, failure to identify group differences may be due to inclusion bias and a type II error. Future studies could include a larger cohort of IBS patients that are followed over time.

At present, it is unclear whether changes in PUFAs profile are state markers, i.e., only present during an illness episode, or trait markers, i.e., also present in symptom-free episodes and in those subjects at risk of affective dysregulation. Alterations in PUFAs profile have been reported in symptom-free first-degree relatives of patients with affective disorders who did not have a previous or current psychiatric diagnosis ${ }^{41}$. Our findings indicate that an association between PUFAs profile and affective dysregulation is also present in a population with a low level of affective dysregulation and without a previous psychiatric diagnosis or first-degree family history of affective disorders. Serotonergic modulators are used in the treatment of both affective disorders as well as IBS, and it has been shown that serotonin is a mediator of brain-gut responses ${ }^{11,42,43}$. In addition, changes in PUFAs are associated with parameters of central serotonergic activity ${ }^{23,41,44}$. Whether these findings may suggest a role for PUFAs profile as a metabolic mediator in the brain-gut interaction remains to be elucidated. 
In addition to the role of PUFAs in serotonergic neurotransmitter systems, PUFAs are the precursors of eicosanoids (prostaglandins, thromboxanes, prostacyclines and leukotrienes), which participate in the regulation of immunological and inflammatory responses ${ }^{45}$. Supplementation with $n-3$ PUFAs leads to the production of eicosanoids with attenuated inflammatory effects in comparison with those produced from n-6 PUFAs ${ }^{46}$. Interestingly, coadministration of $n-3$ PUFAs has been reported to improve affective dysregulation in major depressive disorder ${ }^{47-49}$.

IBS has always been considered a functional gastrointestinal disorder without any signs of structural or metabolic abnormalities, such as inflammation of the gut wall. However, the observations that $d$-IBS may be precipitated by an acute enteric infection and that some d-IBS patients have an increased number of inflammatory cells in the intestinal mucosa have recently prompted consideration of inflammation as a putative basis for symptom generation in d-IBS ${ }^{50-52}$. In this regard, d-IBS may follow a similar pattern of pathogenesis similar to other chronic relapsing inflammatory disorders such as rheumatoid arthritis and chronic obstructive pulmonary disease. Dietary supplements of $n-3$ PUFAs have been reported beneficial in the treatment of many inflammatory conditions, i.e., inflammatory bowel disease (IBD: Crohn's disease and ulcerative colitis), eczema, psoriasis and rheumatoid arthritis ${ }^{53,54}$.

Although not the aim of our study, comparison of the functional bowel disorder IBS with IBD with respect to the influence of PUFAs on the pathophysiological processes and course of the diseases is challenging. In both disorders the brain-gut axis seems to play an important role in the pathophysiology ${ }^{55,56}$. The role of the brain-gut axis, however, has been far better established in IBS than IBD. On the other hand, the role of inflammation, even at a systemic level, is much more pronounced in IBD with regard to the pathophysiology and generation of symptoms. Regarding future research, it would be interesting to obtain intestinal biopsies in IBS patients in order to subdivide IBS patients into groups with and without signs of intestinal inflammation and study the relation with PUFAs profile and affective dysregulation ${ }^{57}$.

Examination of the data concerning an association between n-3 PUFAs and SCL-depression scores revealed two outliers. Because of the relatively small number of subjects and the strong influence of these two patients' scores on the regression model, we considered the model without the two outliers to be a better estimation of the association between total n-3 PUFAs and $S C L$-depression scores ${ }^{58}$. Potential confounders are not expected to explain our findings. IBS patients were successfully matched to controls with respect to age, gender, $\mathrm{BMl}$, alcohol consumption, smoking behaviour, menstrual cycle phase, and contraception. As we did not assess dietary fat intake, we cannot 
exclude that the quantitative and qualitative intake in IBS patients differed from that in the controls ${ }^{59}$. However, we screened all subjects for cholesterolrestricted or weight-reducing diets. In addition, patients were screened for the presence of possible lactose malabsorption, which has been associated with symptoms identical to d-IBS as well as with signs of mental depression and could be associated with malabsorption of relevant nutrients ${ }^{33}$. Furthermore, the absolute FA concentrations as well as the percentages of linoleic acid and $\alpha$-linolenic acid, the precursors of the n-6 and n-3 PUFAs series, did not significantly differ between patients and controls, which suggest no difference in intake, intestinal absorption, or synthesis between patients and controls.

In conclusion, the findings of the present study revealed that affective dysregulation was higher in IBS patients compared with healthy controls and that changes in PUFAs and cholesterol profile were significantly associated with affective dysregulation. These results warrant further studies regarding the role of PUFAs and cholesterol status in the co-occurrence of affective dysregulation and functional gastrointestinal disorders. 


\section{References}

1. Talley NJ, Spiller R. Irritable bowel syndrome: a little understood organic bowel disease? Lancet 2002;360:555-564.

2. Thompson WG, Longstreth GF, Drossman DA, Heaton KW, Irvine EJ, Muller-Lissner SA. Functional bowel disorders and functional abdominal pain. Gut 1999;45 Suppl 2:1143-47.

3. El-Serag $H B$, Olden $K$, Bjorkman $D$. Health-related quality of life among persons with irritable bowel syndrome: a systematic review. Aliment Pharmacol Ther 2002;16:1171-1185.

4. Sandler RS, Everhart JE, Donowitz M, Adams E, Cronin K, Goodman C, Gemmen E, Shah S, Avdic A, Rubin R. The burden of selected digestive diseases in the United States. Gastroenterology 2002;122:1500-1511.

5. Mayer EA. Emerging disease model for functional gastrointestinal disorders. Am J Med 1999; 107:12S-19S.

6. Mayer EA, Gebhart GF. Basic and clinical aspects of visceral hyperalgesia. Gastroenterology 1994;107:271-293.

7. Noyes R, Jr., Cook B, Garvey $M$, Summers R. Reduction of gastrointestinal symptoms following treatment for panic disorder. Psychosomatics 1990;31:75-79.

8. Ford MJ, Miller PM, Eastwood J, Eastwood MA. Life events, psychiatric illness and the irritable bowel syndrome. Gut 1987;28:160-165.

9. Walker EA, Roy-Byrne PP, Katon WJ. Irritable bowel syndrome and psychiatric illness. Am J Psychiatry 1990;147:565-572.

10. Lydiard RB, Fossey MD, Marsh W, Ballenger JC. Prevalence of psychiatric disorders in patients with irritable bowel syndrome. Psychosomatics 1993;34:229-234.

11. Kilkens TO, Honig A, Rozendaal N, Van Nieuwenhoven MA, Brummer RJ. Serotonergic modulators in the treatment of irritable bowel syndrome - influence on psychiatric and gastrointestinal symptoms. Aliment Pharmacol Ther 2003;17:43-51.

12. Houghton LA, Atkinson W, Whitaker RP, Whorwell PJ, Rimmer MJ. Increased platelet depleted plasma 5-hydroxytryptamine concentration following meal ingestion in symptomatic female subjects with diarrhoea predominant irritable bowel syndrome. Gut 2003;52:663-670.

13. Singh RK, Pandey HP, Singh RH. Correlation of serotonin and monoamine oxidase levels with anxiety level in diarrhea-predominant irritable bowel syndrome. Indian J Gastroenterol 2003;22:88-90.

14. Camilleri M, Northcutt AR, Kong S, Dukes GE, McSorley D, Mangel AW. Efficacy and safety of alosetron in women with irritable bowel syndrome: a randomised, placebo-controlled trial. Lancet 2000;355:1035-1040.

15. Walker EA, Roy-Byrne PP, Katon WJ, Li L, Amos D, Jiranek G. Psychiatric illness and irritable bowel syndrome: a comparison with inflammatory bowel disease. Am J Psychiatry 1990;147:1656-1661.

16. Fock KM, Chew CN, Tay LK, Peh LH, Chan S, Pang EP. Psychiatric illness, personality traits and the irritable bowel syndrome. Ann Acad Med Singapore 2001;30:611-614.

17. Maes M, Smith R, Christophe A, Cosyns $P$, Desnyder R, Meltzer H. Fatty acid composition in major depression: decreased omega 3 fractions in cholesteryl esters and increased C20: 4 omega 6/C20:5 omega 3 ratio in cholesteryl esters and phospholipids. J Affect Disord 1996; 38:35-46.

18. Hibbeln JR, Umhau JC, Linnoila M, George DT, Ragan PW, Shoaf SE, Vaughan MR, Rawlings R, Salem N, Jr. A replication study of violent and nonviolent subjects: cerebrospinal fluid metabolites of serotonin and dopamine are predicted by plasma essential fatty acids. Biol Psychiatry 1998;44:243-249.

19. Adams PB, Lawson S, Sanigorski A, Sinclair AJ. Arachidonic acid to eicosapentaenoic acid ratio in blood correlates positively with clinical symptoms of depression. Lipids 1996;31 Suppl: S157-S161.

20. Freeman MP. Omega-3 fatty acids in psychiatry: a review. Ann Clin Psychiatry 2000;12: 159-165. 
21. Morgan RE, Palinkas LA, Barrett-Connor EL, Wingard DL. Plasma cholesterol and depressive symptoms in older men. Lancet 1993;341:75-79.

22. Hibbeln JR, Salem N, Jr. Dietary polyunsaturated fatty acids and depression: when cholesterol does not satisfy. Am J Clin Nutr 1995;62:1-9.

23. Hibbeln JR, Linnoila M, Umhau JC, Rawlings R, George DT, Salem N, Jr. Essential fatty acids predict metabolites of serotonin and dopamine in cerebrospinal fluid among healthy control subjects, and early- and late-onset alcoholics. Biol Psychiatry 1998;44:235-242.

24. Chalon S, Vancassel S, Zimmer L, Guilloteau D, Durand G. Polyunsaturated fatty acids and cerebral function: focus on monoaminergic neurotransmission. Lipids 2001;36:937-944.

25. Horrobin DF, Bennett $C N$. Depression and bipolar disorder: relationships to impaired fatty acid and phospholipid metabolism and to diabetes, cardiovascular disease, immunological abnormalities, cancer, ageing and osteoporosis. Possible candidate genes. Prostaglandins Leukot Essent Fatty Acids 1999;60:217-234.

26. Maes M, Christophe A, Delanghe J, Altamura C, Neels H, Meltzer HY. Lowered omega-3 polyunsaturated fatty acids in serum phospholipids and cholesteryl esters of depressed patients. Psychiatry Res 1999;85:275-291.

27. Drossman DA, Heaton K, Irvine EJ, Muller-Lissner S. Functional bowel disorders and functional abdominal pain. In: Drossman DA, Corazziari E, Talley N, Thompson WG, Whitehead WE, editors. The functional gastrointestinal disorders. Diagnosis, pathophysiology and treatment: A multinational consensus. McLean, VA: Degnon Associates; 2000:351-398.

28. Sheehan D. MINI International neuropsychiatric Interview: Tampa: University of South Florida; 1994.

29. Arrindell WA, Ettema JHM. SCL-90. Een multidimensionale psychopathologie indicator [SCL90. A multidimensional indicator of psychopathology]. In. Lisse, The Netherlands: Swets \& Zeitlinger; 1986.

30. Mykletun A, Stordal E, Dahl AA, Hospital Anxiety and Depression (HAD) scale: factor structure, item analyses and internal consistency in a large population. $\mathrm{Br} \mathrm{J}$ Psychiatry 2001; 179:540-544.

31. Hamilton M. Development of a rating scale for primary depressive illness. $\mathrm{Br} \mathrm{J}$ Soc Clin Psychol 1967;6:278-296.

32. Hermans $M M$, Brummer RJ, Ruijgers $A M$, Stockbrugger RW. The relationship between lactose tolerance test results and symptoms of lactose intolerance. Am J Gastroenterol 1997; 92:981-984.

33. Ledochowski $M$, Sperner-Unterweger $B$, Fuchs $D$. Lactose malabsorption is associated with early signs of mental depression in females: a preliminary report. Dig Dis Sci 1998;43: 2513-2517.

34. Sobczak S, Riedel WJ, Booij I, Aan Het Rot M, Deutz NE, Honig A. Cognition following acute tryptophan depletion: difference between first-degree relatives of bipolar disorder patients and matched healthy control volunteers. Psychol Med 2002;32:503-515.

35. Zeleniuch-Jacquotte $A$, Chajes $V$, Van Kappel AL, Riboli $E$, Toniolo P. Reliability of fatty acid composition in human serum phospholipids. Eur J Clin Nutr 2000;54:367-372.

36. Folch $J$, Lees $M$, Sloane Stanley $G H$. A simple method for the isolation and purification of total lipides from animal tissues. J Biol Chem 1957;226:497-509.

37. Kaluzny MA, Duncan LA, Merritt MV, Epps DE. Rapid separation of lipid classes in high yield and purity using bonded phase columns. J Lipid Res 1985;26:135-140.

38. Hair JF. Multivariate data analysis. New Yersey: Prentice-Hall, Inc. A Simon \& Schuster Company; 1998.

39. Prior $A$, Maxton DG, Whorwell PJ. Anorectal manometry in irritable bowel syndrome: differences between diarrhoea and constipation predominant subjects. Gut 1990;31:458-62.

40. Elsenbruch $S$, Orr WC. Diarrhea- and constipation-predominant IBS patients differ in postprandial autonomic and cortisol responses. Am J Gastroenterol 2001;96:460-466.

41. Sobczak S, Honig A, Christophe A, Maes M, Helsdingen RW, De Vriese SA, Riedel WJ. Lower high-density lipoprotein cholesterol and increased omega-6 polyunsaturated fatty acids in first-degree relatives of bipolar patients. Psychol Med 2004;34:103-112. 
42. Kilkens TO, Honig A, van Nieuwenhoven MA, Riedel WJ, Brummer RJ. Acute tryptophan depletion affects brain-gut responses in irritable bowel syndrome patients and controls. Gut 2004;53:1794-1800.

43. Kim DY, Camilleri M. Serotonin: a mediator of the brain-gut connection. Am J Gastroenterol 2000;95:2698-2709.

44. Kodas E, Galineau L, Bodard S, Vancassel S, Guilloteau D, Besnard JC, Chalon S. Serotoninergic neurotransmission is affected by $n-3$ polyunsaturated fatty acids in the rat. $J$ Neurochem 2004;89:695-702.

45. Calder PC. Polyunsaturated fatty acids, inflammation, and immunity. Lipids 2001;36: 1007-1024.

46. Calder PC. N-3 polyunsaturated fatty acids and inflammation: from molecular biology to the clinic. Lipids 2003;38:343-352.

47. Su KP, Huang SY, Chiu CC, Shen WW. Omega-3 fatty acids in major depressive disorder. A preliminary double-blind, placebo-controlled trial. Eur Neuropsychopharmacol 2003;13: 267-271.

48. Puri BK, Counsell SJ, Richardson AJ, Horrobin DF. Eicosapentaenoic acid in treatmentresistant depression. Arch Gen Psychiatry 2002;59:91-92.

49. Peet $M$, Horrobin DF. A dose-ranging exploratory study of the effects of ethyleicosapentaenoate in patients with persistent schizophrenic symptoms. J Psychiatr Res 2002;36:7-18.

50. Collins SM, Piche T, Rampal P. The putative role of inflammation in the irritable bowel syndrome. Gut 2001;49:743-745.

51. Barbara $G$, De Giorgio $R$, Stanghellini $V$, Cremon $C$, Corinaldesi R. A role for inflammation in irritable bowel syndrome? Gut 2002;51 Suppl 1:i41-44.

52. Spiller RC. Postinfectious irritable bowel syndrome. Gastroenterology 2003;124:1662-1671.

53. Gil A. Polyunsaturated fatty acids and inflammatory diseases. Biomed Pharmacother 2002; 56:388-396.

54. Geerling BJ, v Houwelingen AC, Badart-Smook A, Stockbrugger RW, Brummer RJ. Fat intake and fatty acid profile in plasma phospholipids and adipose tissue in patients with Crohn's disease, compared with controls. Am J Gastroenterol 1999;94:410-417.

55. Hollander D. Inflammatory bowel diseases and brain-gut axis. J Physiol Pharmacol 2003;54 Suppl 4:183-190.

56. Mertz HR. Overview of functional gastrointestinal disorders: dysfunction of the brain-gut axis. Gastroenterol Clin North Am 2003;32:463-476.

57. Dunlop SP, Jenkins $D$, Neal KR, Spiller RC. Relative importance of enterochromaffin cell hyperplasia, anxiety, and depression in postinfectious IBS. Gastroenterology 2003;125: 16511659.

58. Cohen J, Cohen P. Applied Multiple Regression/Correlation Analyses for the Behavioral Sciences: Erlbaum, Hillsdale, NJ; 1983.

59. Arab L. Biomarkers of fat and fatty acid intake. J Nutr 2003;133 Suppl 3:925S-932S. 


\section{Chapter 5}

Acute tryptophan depletion affects brain-gut responses in irritable bowel syndrome patients and controls

Kilkens TO, Honig A, van Nieuwenhoven MA, Riedel WJ, Brummer RJ Gut 2004; 53: 1794-1800 
$90 \mid$ Chapter 5

\section{Abstract}

\section{Background}

Serotonin, a key denominator of the brain-gut axis, is involved in the regulation of gastrointestinal motility, secretion, and perception as well as cognition and mood.

Aim

To assess the effects of an acutely lowered serotonin synthesis, using the acute tryptophan depletion (ATD) method, on visceral perception, affective memory performance, and mood in diarrhoea-predominant irritable bowel syndrome patients (d-IBS) and controls.

Methods

In a randomised, double-blind, crossover design, fourteen d-IBS patients and fourteen matched controls were studied under ATD and placebo conditions, respectively. Perception of urge and pain was scored during rectal distensions. Affective memory performance, mood, and biochemical parameters of serotonergic metabolism were simultaneously assessed.

Results

ATD significantly decreased plasma tryptophan $\left(\begin{array}{llllll}67.0 & (2.0) & \text { vs. } 24.9 & (2.0) \mu \mathrm{mol} / \mathrm{l})\end{array}\right.$ and 5-hydroxyindole acetic acid concentrations (29.9 (1.0) vs. $15.8(0.6)$ nmol/l). ATD was associated with significantly increased urge scores specifically in the lower pressure range and overall increased pain scores. ATD significantly lowered the perceptual threshold for first perception compared with placebo (patients $10.6(1.2)$ vs. $13.6(0.8) \mathrm{mmHg}$, controls $12.6(1.3)$ vs. $15.7(1.2)$ $\mathrm{mmHg}$ ), but not for maximal tolerable discomfort (patients $50.5(3.6)$ vs. $51.6(3.3) \mathrm{mmHg}$, controls $50.9(3.3)$ vs. $48.8(2.9) \mathrm{mmHg}$ ). ATD induced a significant shift in affective memory bias towards preferential loss of positive material but no significant changes in mood. ATD did not differentially affect the patient or control group.

\section{Conclusions}

We have provided evidence that serotonergic modulation by ATD affects both visceral perception as well as cognition in d-IBS and controls. Simultaneous measurement of brain and gut function and the application of ATD contribute to the elucidation of the complex pathophysiology of IBS. 
Acute tryptophan depletion and the brain-gut axis $\mid 91$

\section{Introduction}

The pathophysiology of irritable bowel syndrome (IBS) is not fully understood. A multi-component conceptual model of IBS has been postulated, involving physiological, affective, cognitive, and behavioural factors'. The 'brain-gut axis' is a theoretical model describing the bidirectional neural pathways linking cognitive and emotional centres in the brain to neuroendocrine centres, the enteric nervous system, and the immune system, and plays a major role in the concept of IBS ${ }^{2}$. IBS is associated with visceral hypersensitivity and with a high co-occurrence of psychiatric symptoms, in particular affective dysregulation ${ }^{3-5}$.

Serotonin (5-HT) is a biogenic amine that functions as a neurotransmitter and is located predominantly in the gastrointestinal (GI) tract $(80 \%$ of body $5-\mathrm{HT})$. Approximately $5 \%$ is located in the brain and cerebrospinal fluid (CSF). 5 -HT is peripherally involved in the regulation of $\mathrm{GI}$ secretion, motility, and perception, whereas in the central nervous system (CNS) it plays a role in the regulation of mood and cognition $n^{6,7}$. Manipulation of serotonergic activity by application of 5-HT modulators has been used in the treatment of both affective disorders and $\mathrm{IBS}^{8-11}$. Hence $5-\mathrm{HT}$ is a key denominator of the 'brain-gut axis' 10,12 .

Disturbed serotonergic metabolism seems especially prevalent in the diarrhoea-predominant type of IBS (d-IBS) ${ }^{13-15}$. Increased postprandial plasma $5-\mathrm{HT}$ levels in d-IBS patients and increased numbers of 5-HT containing enterochromaffin (EC) cells in postinfectious IBS (PI-IBS) have been observed $^{5,16,17}$. In contrast with the known effects of 5-HT on Gl motility and secretion, the role of 5-HT in visceral perception to volume based distensions has been less well established ${ }^{7,18}$.

An acute decrease in 5-HT synthesis can be achieved by means of the acute tryptophan depletion (ATD) method. ATD selectively lowers 5-HT synthesis, between four and seven hours after oral administering of an amino acid mixture devoid of the 5-HT precursor tryptophan ${ }^{19}$. In human subjects, substantial reductions of brain 5-HT synthesis and decreased levels of CSF 5-hydroxyindole acetic acid (5-HIAA), the major metabolite of 5-HT, have been demonstrated ${ }^{20}$. Peripherally, the ATD method leads to a $60-70 \%$ decrease in plasma tryptophan levels ${ }^{21}$.

ATD has been used over the past decade in the psychiatric setting to investigate the role of the central-5-HT system in patients with affective disorders (depression and anxiety) and their first-degree relatives. It serves as a biological model for inducing symptoms of depression such as impaired mood and affective memory performance (loss of words with a positive 
connotation $)^{22-24}$. In addition, recent findings at our department showed that ATD also affects $\mathrm{Gl}$ physiology by delaying gastric emptying in healthy females ${ }^{25}$.

The above findings indicate that the ATD method offers a potential technique to study the contribution of 5-HT to regulation of the 'brain-gut axis'. This paper describes the effects of serotonergic modulation, using the ATD method, at the level of both the brain and the gut, in patients with d-IBS and healthy controls. We applied this novel model to investigate whether: (1) ATD influences visceral perception during rectal distensions and (2) whether ATD simultaneously influences affective memory performance and mood. Based on the known effects of ATD on the CNS and given the lines of evidence that (1) IBS is associated with enhanced visceral perception ${ }^{4}$ and lowered mood ${ }^{10}$ and (2) lowered mood is associated with decreased serotonergic activity ${ }^{6}$, we hypothesized that decreased serotonergic synthesis would be associated with enhanced visceral perception and impaired affective memory performance and mood.

Materials and methods

\section{Subjects}

All subjects were screened which involved a standardized psychiatric examination using the mini international neuropsychiatric interview (MINI) ${ }^{26}$ to determine the present psychiatric state. General psychological state was assessed using the 17-item Hamilton Depression Rating Scale (HAM-D17) ${ }^{27}$, the Dutch version of the Symptom Check List $(\mathrm{SCL}-90)^{28}$ and the Hospital Anxiety and Depression Scale (HADS) ${ }^{29}$. Physical health was assessed by means of a standard physical examination and a urine test. A pregnancy test was performed in all female subjects. Intelligence was estimated using the Groninger Intelligence Test $(G I T)^{30}$. Prior to the test days a training version of the affective memory performance test was practiced to minimize learning effects.

The Medical Ethics Committee of the University Hospital Maastricht approved the study protocol and all subjects gave written informed consent before start of the study. The required number of included subjects was based on pilot experiments and an estimated effect size of 1.0 (calculated from the standardized mean difference between ATD and placebo conditions with respect to the primary outcome parameter), with $\beta=0.2$ and $\alpha=0.05$. Fourteen patients fulfilled the diagnosis of $d$-IBS according to the ROME II criteria, 
diagnosed by an experienced gastroenterologist ${ }^{31}$. Exclusion criteria were abdominal surgery (other than appendectomy and cholecystectomy), use of medications other than oral contraceptives within fourteen days prior to testing, lactose intolerance ${ }^{32}$, a positive first-degree psychiatric family history ${ }^{21}$, any history of psychiatric disease or use of psychoactive medication, premenstrual syndrome, dieting, pregnancy, or lactation, excessive alcohol intake $(>20$ alcoholic consumptions per week) and hypertension (diastolic $>100 \mathrm{mmHg}$, systolic $>170 \mathrm{mmHg}$ ), respectively.

Fourteen healthy matched control subjects were included. The same exclusion criteria were used. Additional exclusion criteria for the control subjects were current or history of $\mathrm{Gl}$ disorder, current psychiatric or psychological symptomatology defined as a diagnosis on the MINI, HAM-D17 scores above 18 , global severity index score on the SCL-90 for females $\geq 150$ and for males $\geq 131$, or HADS scores $\geq 8$.

Table 5.1 Subject characteristics (mean (SE)).

\begin{tabular}{lccc}
\hline & $\begin{array}{c}d-\text { IBS patients } \\
n=14\end{array}$ & $\begin{array}{c}\text { Controls } \\
n=14\end{array}$ & $\begin{array}{c}\text { Patients vs. Controls } \\
\text { p value }\end{array}$ \\
\hline Sex (F/M) ( $n$ ) & $8 / 6$ & $8 / 6$ & - \\
Oral contraceptives $(n)$ & $6 / 8$ & $6 / 8$ & - \\
Age & $34.1(3.0)$ & $33.9(3.8)$ & 0.7 \\
Intelligence quotient & $106(3.6)$ & $110(3.0)$ & 0.5 \\
Body mass index & $23.3(1.0)$ & $22.9(0.7)$ & 0.7 \\
Alcohol units/day & $0.61(0.2)$ & $0.52(0.1)$ & 0.8 \\
Cigarettes/day & $2.71(1.2)$ & $1.43(0.9)$ & 0.2 \\
Diagnosis on MINI & $4^{\mathrm{c}}$ & 0 & - \\
HAM-D17 & $3.64(1.1)$ & $0.86(0.3)$ & $0.03^{\mathrm{a}}$ \\
SCL-90 (GSI) & $114(6.3)$ & $97.4(2.3)$ & $0.02^{\mathrm{a}}$ \\
HADS (total) & $5.14(0.9)$ & $3.93(0.9)$ & 0.34 \\
\hline
\end{tabular}

d-IBS, diarrhoea-predominant IBS; MINI, Mini International Neuropsychiatric Interview; HAM-D17, 17-item Hamilton Depression Scale; SCL-90, Symptom Check List; GSI, Global Severity Index; HADS, Hospital Anxiety and Depression Scale. ${ }^{a}$ Significant difference between groups. ${ }^{b}$ Independent samples $t$-tests comparing d-IBS patients versus control subjects. ${ }^{\circ}$ Four subjects had a psychiatric diagnosis: depression, agoraphobia, social phobia, and anxiety disorder, respectively.

\section{Study design}

The study was conducted following a randomised, placebo-controlled, doubleblind, crossover design. The two test days were separated by a minimum of seven days. All women were tested in the follicular phase of the menstrual cycle or while taking oral contraception ${ }^{33}$. All subjects were tested within three months to avoid possible seasonal variation ${ }^{34}$. They were asked to abstain from heavy physical exercise and consumption of alcoholic beverages the day prior to their visit. All subjects attended the laboratory twice after an overnight fast (after 10:00 PM no eating, drinking or smoking was allowed) at 8:00 AM. At 
$94 \mid$ Chapter 5

$t=8: 30$ the amino acid drink (ATD or placebo mixture) was administered. Blood samples were taken at $t=8: 00$ and $t=15: 00$. Affective memory performance and visceral perception were assessed at $t=12: 30$ and $t=14: 00$, respectively. Mood was assessed six times during each test day at $t=8: 00,10: 30,12: 30,14: 00$, 14:30, and $t=15: 00$ (Figure 5.1).

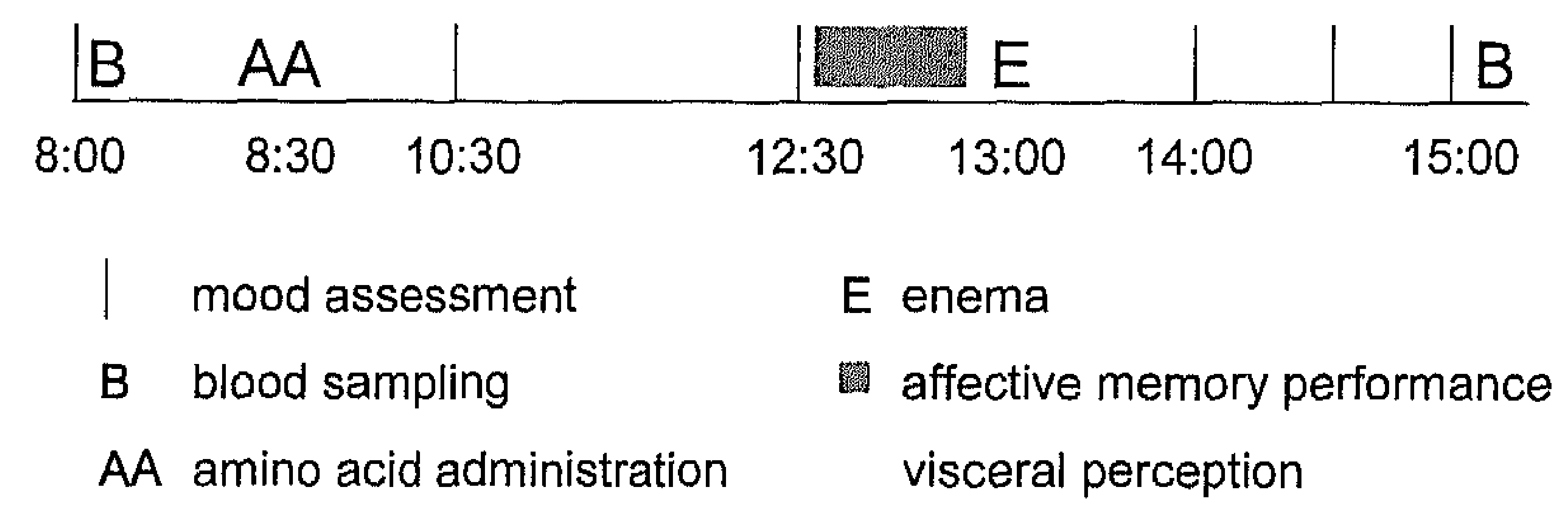

Figure 5.1 Schematic representation of a test day (flow chart).

\section{Tryptophan depletion}

The Department of Pharmacy of the University Hospital prepared the amino acid mixtures. The tryptophan deficient amino acid (ATD) mixture consisted of fifteen amino acids, including five large neutral amino acids (LNAAs) ${ }^{21}$. The placebo mixture contained the same amino acids plus 3 grams of tryptophan in order to prevent a decrease in tryptophan levels ${ }^{21}$. The mixture was dissolved in $200 \mathrm{ml}$ of tap water and subjects were instructed to ingest the mixture as quickly as possible.

\section{Visceral perception}

The bowel preparation consisted of a tap water enema administered one hour before the barostat procedure. The rectal probe was lubricated and placed into the rectum so that the attached end of the bag was $3 \mathrm{~cm}$ from the anal sphincter. The probe consisted of a $700 \mathrm{ml}$ polyethylene bag secured on a rectal catheter (external diameter $=18$ French). The catheter was connected to the electronic distension device (Electronic Barostat; Distender Series II; G \& J Electronics Inc., Toronto, Ontario, Canada) using the Protocol Plus Deluxe Software (G \& J Electronics Inc.). The barostat inflation rate was $50 \mathrm{ml} / \mathrm{s}$ to a constant pressure plateau. Subjects were not given any auditory or visual clues that they were to receive a stimulus and were not told the exact nature of the distension protocol. To reduce the influence of adipose tissue mass and abdominal wall tone, subjects were all placed in the left lateral position. 
Acute tryptophan depletion and the brain-gut axis $\mid 95$

In order to unfold the rectal probe, a five minute $10 \mathrm{mmHg}$ distension followed by a one minute $20 \mathrm{mmHg}$ distension was administered. Subsequently, the barostat protocol consisted of intermittent semi-random staircase distensions of 60 seconds duration $(15,10,25,20 \mathrm{mmHg}$, etcetera) separated by an interval of 30 seconds baseline pressure ${ }^{35}$. The end point to stop the series of distensions was the perceptual threshold for maximal tolerable discomfort or if the safety value of the maximal volume of $600 \mathrm{ml}$ was exceeded. During each distension (after thirteen seconds of distension) subjects were asked to report their perception of urge and pain. Urge was scored on a panel (Deluxe Perception Panel; G\&J Electronics Inc.) with six buttons labelled: 1, not perceptible; 2 , first perception; 3 , minor urge to defecate; 4 , normal urge to defecate; 5 , strong urge to defecate; and 6 , maximal tolerable urge ${ }^{35}$. Pain was scored using a $100 \mathrm{~mm}$ visual analogue scale (no pain-maximal tolerable pain). Rectal volumes were measured at the end of each distension (after 60 seconds distension). Volume was corrected for air compressibility. Rectal compliance $(\Delta \mathrm{V} / \Delta \mathrm{P} ; \mathrm{P}=10-45 \mathrm{mmHg})$ was estimated for each subject.

\section{Cognition and mood}

The affective memory test consisted of a list of 30 emotionally loaded stimulus words (twelve positive, twelve negative, and six neutral) ${ }^{23}$. All 30 words on the list were consecutively presented three times on a computer screen. Each presentation of the list ended with a free recall of the words. Recall scores were summed to comprise the total immediate recall score. After the third presentation, subjects performed a 30 minute distraction task and subsequently subjects were requested to recall as many of the previously learned words as possible (delayed recall). Outcome measures were the percentages of positive, negative, and neutral words recalled of the total immediate recall and delayed recall score ${ }^{22}$. Mood was assessed with visual analogue scales adapted from the profile of mood states (POMS) ${ }^{36}$. It consisted of 32 items describing bipolar mood adjectives (for example, happy - sad). Items were grouped to form measures of five mood dimensions: depression, tension, vigour, anger, and fatigue, respectively.

\section{Biochemical parameters}

Total plasma tryptophan concentrations and the tryptophan/ $L$ LNAAs ratios (sum of tyrosine, valine, leucine, isoleucine, and phenylalanine concentrations) were determined. This ratio provides an estimate of tryptophan uptake into the brain and consequently central $5-\mathrm{HT}$ synthesi ${ }^{37}$. In addition, concentrations of platelet 5-HT, platelet poor plasma (ppp) 5-HT, its major metabolite 5-HIAA, and $5-\mathrm{HT}$ turnover (5-HIAA:5-HT) were determined as markers of peripheral 5-HT metabolism. Blood was sampled in EDTA Vacutainer tubes. Samples for 
$96 \mid$ Chapter 5

tryptophan and LNAAs were immediately placed on ice and centrifuged within 30 minutes $\left(10\right.$ minutes, $\left.900 \mathrm{~g}, 4^{\circ} \mathrm{C}\right)$. For platelet $5-\mathrm{HT}$ measurement, whole blood was used. In addition, an aliquot of blood kept at room temperature was used for whole blood platelet counting. Samples for ppp 5-HT and 5-HIAA were kept at room temperature and centrifuged (20 minutes, $\left.2600 \mathrm{~g}, 20^{\circ} \mathrm{C}\right)$. As a control of the ppp preparation procedure, platelets were also counted in the ppp using a Coulter Counter (Coulter MD Series, Coulter Corporation, Hialeah, Florida, USA). All samples were stored at $-80^{\circ} \mathrm{C}$ until analysis. Plasma tryptophan, $\mathrm{LNAAs}^{38,39}$, ppp 5-HT, and 5-HIAA ${ }^{40,41}$ samples were analysed using high performance liquid chromatography, as previously described.

\section{Statistical analysis}

The primary outcome parameter was visceral perception. Secondary outcome parameters were rectal compliance, affective memory performance, and mood. Data are presented as mean (SE). Differences in subject characteristics were analysed using independent-samples $t$-tests. Outcome variables were analysed using analysis of variance design (ANOVA) for repeated measures. For visceral perception thresholds (first perception and maximal tolerable discomfort) and affective memory performance, the within subjects factor was treatment (two levels: ATD, placebo). For biochemical parameters, the within subjects factors were treatment (two levels: ATD, placebo) and time (two levels: $t=8: 00,15: 00)$. Urge and pain perception were analysed in the range 10$45 \mathrm{mmHg}$, as our total number of subjects became too small for adequate statistical analysis at pressures above $45 \mathrm{mmHg}$. For visceral urge, pain perception, and mood analyses, within subjects factors were treatment (two levels: ATD, placebo), pressure (eight levels: 10, 15, 20, 25, 30, 35, 40, $45 \mathrm{mmHg}$ ) and time (six levels: $t=8: 00,10: 30,12: 30,14: 00,14: 30,15: 00)$. The between subjects factor for all outcome variables was diagnosis of d-IBS (two levels: d-IBS, controls). In case of a slight violation of sphericity, the Greenhouse-Geisser correction was applied. If indicated, post hoc analyses were performed for certain time points and Bonferroni corrections were applied. A $p$ value of less than 0.05 was considered statistically significant. Statistical analyses were performed using the SPSS 10.0 for Windows software package.

\section{Results}

\section{Visceral perception}

Urge and pain (Figure 5.2, 5.3). Urge and pain scores increased significantly with increasing pressure $[F(7,13)=199.0, p<0.001 ; F(7,12)=7.1, p<0.01]$, respectively. For the urge scores, there was a strong significant pressure $x$ 
diagnosis of $d-$ IBS interaction $[F(7,133)=3.2, p<0.005]$, indicating that patients had relatively higher urge scores in the lower pressure range and relatively lower urge scores in the higher pressure range compared with controls (Figure 5.2). Post hoc analysis indicated that in the lower pressure range $(10-15 \mathrm{mmHg})$, patients had significantly higher urge scores compared with controls $[F(1,26)=4.6, p<0.05]$.

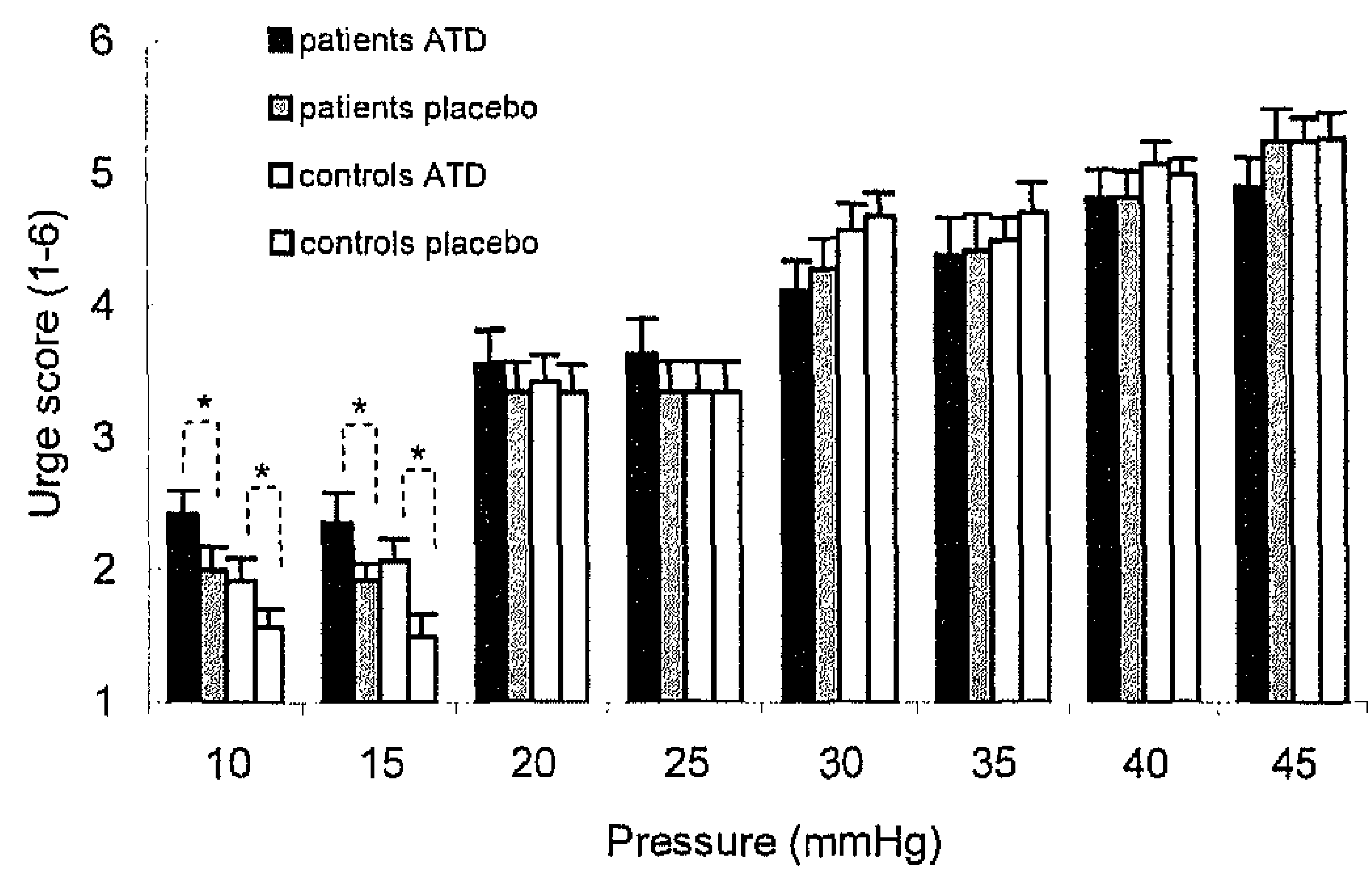

Figure 5.2 Pressure-urge scores (mean (SE)) during intermittent pressure distension of the rectum in diarrhoea-predominant irritable bowel syndrome (d-IBS) patients and control subjects, during acute tryptophan depletion (ATD) and placebo. Overall, ATD did not significantly affect urge scores compared with placebo. However, post hoc analysis indicated that in the lower pressure range, ATD was significantly associated with increased urge scores compared with placebo $(p<0.001)(10-15 \mathrm{mmHg})$.

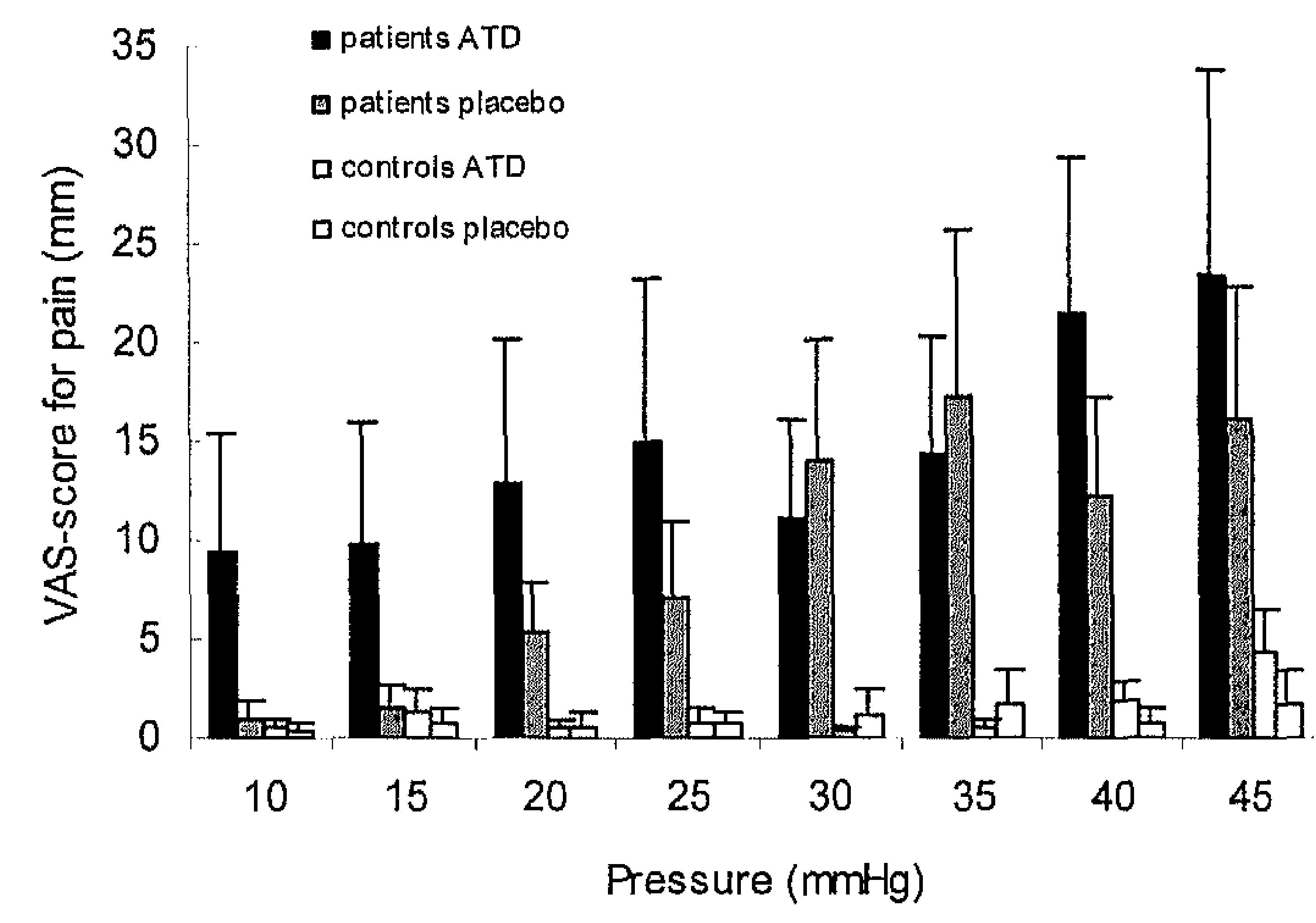

Figure 5.3 Pressure-pain scores (mean (SE)) during intermittent pressure distension of the rectum in diarrhoea-predominant irritable bowel syndrome (d-IBS) patients and control subjects, during acute tryptophan depletion (ATD) and placebo. Overall, patients showed significantly increased pain scores compared with controls $(p=0.02)$. ATD was significantly associated with increased pain scores $(p=0.04)$. 
$98 \mid$ Chapter 5

Patients experienced overall significantly more pain compared with controls $[F(1,17)=4.7, p<0.05]$ and their pain scores increased significantly more with increasing pressure compared with controls (pressure $x$ diagnosis of $d-I B S$ interaction) $[F(9,119)=7.1, \quad p<0.001]$, which confirms the presence of hypersensitivity in our d-IBS population.

Overall, there was a significant pressure $x$ treatment interaction for the perception of urge $[F(7,133)=3.1, p<0.01]$, indicating that in the lower pressure range ATD enhanced the perception of urge whereas this enhancing effect of ATD was no longer present in the higher pressure range (Figure 5.2). Post hoc analysis showed significantly increased urge scores in the lower pressure range $(10-15 \mathrm{mmHg})$ during ATD compared with placebo $[F(1,26)=23.6$, $p<0.001]$.

ATD was significantly associated with increased overall pain scores compared with placebo $[F(1,17)=3.4, p<0.05]$. For the pain scores there was, in contrast with the urge scores, no significant pressure $x$ treatment interaction $(p>0.3)$, indicating that the effect of ATD was not specific for the higher or lower pressure range (Figure 5.3). The effects of ATD compared with placebo on urge and pain scores did not differ significantly between patients and controls.

Perceptual thresholds (Table 5.2). Patients tended to have lower first perception thresholds compared with controls although this did not reach significance $[F(1,26)=2.5, p=0.06]$. Thresholds for maximal tolerable discomfort did not differ between patients and controls $[F(1,26)=0.1, p=0.8]$.

ATD significantly lowered the perceptual threshold for first perception compared with placebo $[F(1,26)=9.03, p=0.003]$. ATD did not significantly influence the perceptual threshold for maximal tolerable discomfort $[F(1,26)=0.06, p=0.8]$ (Table 5.2). The effects of ATD compared with placebo on perceptual thresholds did not differ significantly between patients and controls $(p>0.4)$.

Table 5.2 Perceptual thresholds (mean (SE)) in diarrhoea-predominant irritable bowel syndrome (d-IBS) patients and control subjects during acute tryptophan depletion (ATD) and placebo.

\begin{tabular}{llccc}
\hline Pressure threshold (mmHg) & & d-lBS patients & Controls & $\begin{array}{c}\text { ATD vs. Placebo } \\
\text { p value }\end{array}$ \\
\hline First perception & ATD & $10.6(1.2)$ & $12.6(1.3)$ & 0.003 \\
& Placebo & $13.6(0.8)$ & $15.7(1.2)$ & \\
Maximal tolerable discomfort & ATD & $50.5(3.6)$ & $50.9(3.3)$ & 0.8 \\
& Placebo & $51.6(3.3)$ & $48.8(2.9)$ & \\
\hline
\end{tabular}


Rectal compliance (Figure 5.4). The pressure-volume curves differed significantly between patients and controls $[F(1,20)=5.7, p<0.05]$ (Figure 5.4). There was no significant difference in pressure-volume relation or apparent rectal compliance during ATD compared with placebo $[F(1,20)=0.09, p=0.8$; $F(1,26)=0.03, p=0.9$, respectively].

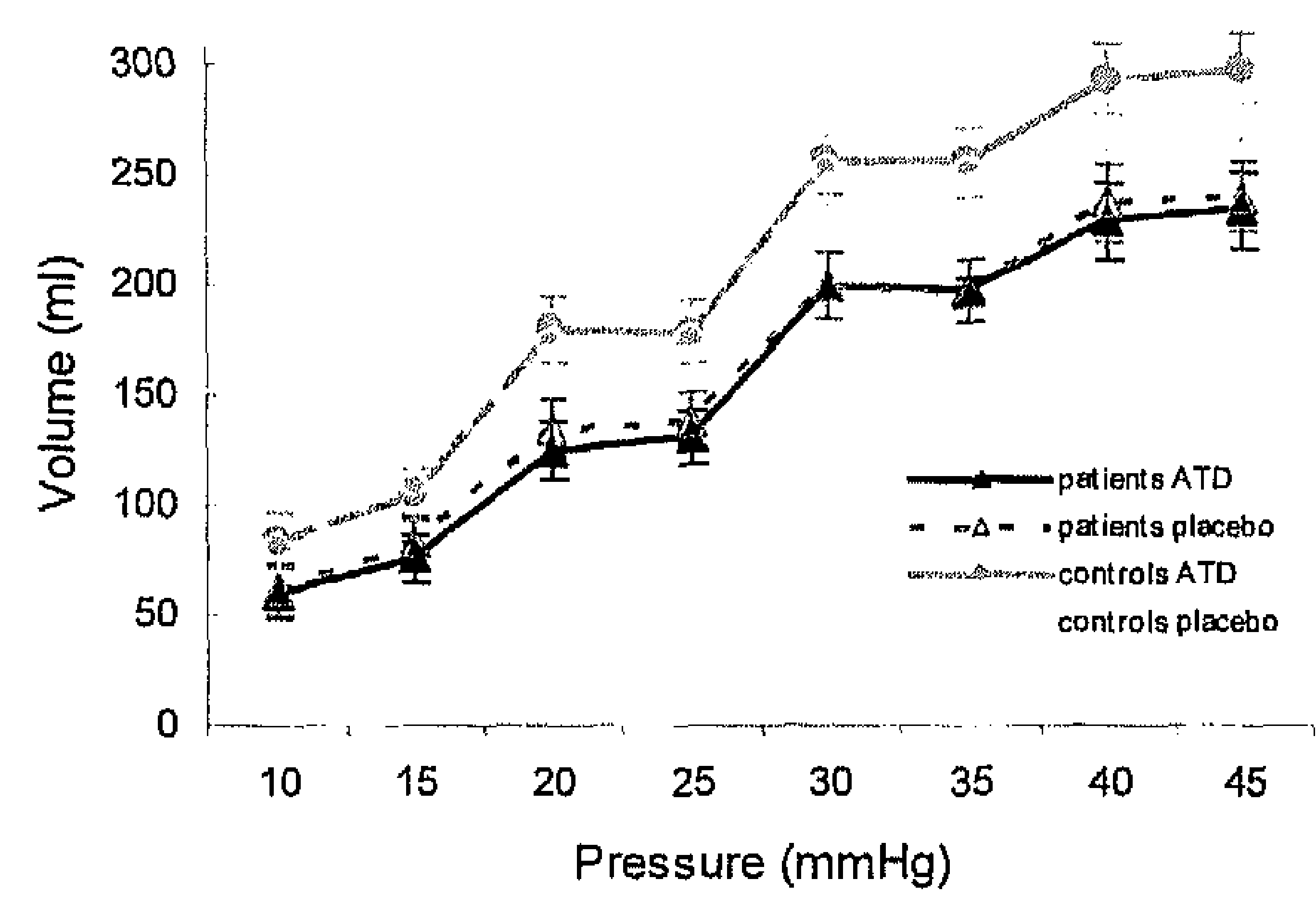

Figure 5.4 Pressure-volume curves (mean (SE)) during intermittent pressure distension of the rectum in diarrhoea-predominant irritable bowel syndrome (d-IBS) patients and control subjects, during acute tryptophan depletion (ATD) and placebo. Patients showed significantly decreased rectal volumes compared with controls $(p=0.02)$. ATD did not significantly influence pressure-volume relations.

\section{Cognition and mood}

Immediate and delayed memory performance as well as percentages of recalled positive and negative words did not significantly differ between patients and controls $(p>0.3)$. However, patients tended to have greater loss of delayed recalled neutral words compared with controls $[F(1,26)=9.5, p=0.06]$ (Table 5.3). 
$100 \mid$ Chapter 5

Table 5.3 Influence of acute tryptophan depletion (ATD) on affective memory performance, in diarrhoea-predominant irritable bowel syndrome (d-IBS) patients and control subjects. Mean (SE) percentages of positive, negative, and neutral words recalled immediately (total immediate recall) and recalled after a delay of 30 minutes (delayed recall).

\begin{tabular}{|c|c|c|c|c|}
\hline & & d-IBS patients & Controls & $\begin{array}{c}\text { ATD vs. Placebo } \\
\text { p value }\end{array}$ \\
\hline \multicolumn{5}{|c|}{ Total immediate recall } \\
\hline \multirow[t]{2}{*}{ Positive words } & ATD & $38.4(4.1)$ & $42.4(4.2)$ & 0.06 \\
\hline & Placebo & $45.3(3.7)$ & $48.9(4.4)$ & \\
\hline \multirow[t]{2}{*}{ Negative words } & ATD & $41.7(2.2)$ & $44.4(3.3)$ & 0.86 \\
\hline & Placebo & $42.0(4.2)$ & $43.4(4.3)$ & \\
\hline \multirow[t]{2}{*}{ Neutral words } & ATD & $44.8(4.3)$ & $50.1(5.2)$ & 0.20 \\
\hline & Placebo & $46.9(4.4)$ & $56.6(3.8)$ & \\
\hline \multicolumn{5}{|l|}{ Delayed recall } \\
\hline \multirow[t]{2}{*}{ Positive words } & ATD & $31.6(5.6)$ & $43.5(7.4)$ & $<0.005^{\mathrm{a}}$ \\
\hline & Placebo & $46.1(5.6)$ & $52.9(7.0)$ & \\
\hline \multirow[t]{2}{*}{ Negative words } & ATD & $41.4(4.7)$ & $45.3(6.1)$ & 0.40 \\
\hline & Placebo & $44.0(6.8)$ & $48.2(7.3)$ & \\
\hline \multirow[t]{2}{*}{ Neutral words } & ATD & $34.6(5.9)$ & $57.5(8.4)$ & 0.31 \\
\hline & Placebo & $48.6(7.4)$ & $57.9(8.7)$ & \\
\hline
\end{tabular}

a Significant after Bonferroni correction

ATD was significantly associated with impaired immediate and delayed recall performance $[F(1,26)=3.3, \quad p<0.05 ; \quad F(1,26)=9.5, \quad p<0.005$, respectively] compared with placebo. Impaired delayed recall was due to impaired recall of positive words $[F(1,26)=10.7, p<0.005]$. Delayed recall of negative and neutral words was not significantly affected by ATD.

ATD did not differentially affect the patient or control group ( $p>0.3)$. There were no significant differences in mood between groups or treatment conditions ( $p$ values $>0.2$ ).

\section{Biochemical parameters}

Biochemical parameters did not significantly differ between groups or treatment conditions at baseline. There were no significant correlations between baseline biochemical values and perception scores under placebo conditions. Tryptophan concentrations as well as tryptophan $/ \Sigma L N A A s$ ratios decreased significantly over time during ATD compared with placebo $[F(1,26)=252.5$, $p<0.001 ; F(1,26)=164.8, p<0.001$, respectively]. ATD did not significantly influence platelet 5-HT or ppp 5-HT concentrations (Table 5.4). 5-HIAA concentrations and ppp 5-HT turnover decreased significantly under ATD conditions compared with placebo $[F(1,26)=338, p<0.001 ; F(1,26)=11, p<0.01$, respectively]. The change in biochemical parameters induced by ATD did not significantly differ between patients and controls $(p>0.5)$ although a trend towards a differential effect of ATD on ppp 5-HT concentrations was observed $[F(1,26)=3.2 p=0.09]$. 
Table 5.4 Biochemical parameters at $t=8: 00$ and $t=15: 00$ (mean (SE)) during acute tryptophan depletion (ATD) and placebo, in the diarrhoea-predominant irritable bowel syndrome (d-IBS) patients and control subjects.

\begin{tabular}{|c|c|c|c|c|c|c|}
\hline & & \multicolumn{2}{|c|}{ d-IBS patients } & \multicolumn{2}{|c|}{ Controls } & \multirow{2}{*}{$\begin{array}{l}\text { ATD vs. } \\
\text { Placebo } \\
\text { p value }\end{array}$} \\
\hline & & $t=8: 00$ & $t=15: 00$ & $t=8: 00$ & $t=15: 00$ & \\
\hline \multirow[t]{2}{*}{ Tryptophan $(\mu \mathrm{mol} / \mathrm{l})$} & ATD & $65.6(3.0)$ & $23.1(2.4)$ & $68.4(2.7)$ & $26.7(3.1)$ & $<0.001$ \\
\hline & Placebo & $62.8(2.0)$ & $91.0(6.0)$ & $65.2(2.1)$ & $100(5.7)$ & \\
\hline \multirow{2}{*}{ Tryptophan/ELNAAs $(x 100)$} & ATD & $11.9(0.7)$ & $2.30(0.3)$ & $12.1(0.7)$ & $2.30(0.2)$ & $<0.001$ \\
\hline & Placebo & $11.4(0.5)$ & $9.70(0.6)$ & $11.4(0.5)$ & $10.3(0.8)$ & \\
\hline \multirow[t]{2}{*}{ Platelet 5-HT (nmol/10 platelets) } & ATD & $4.40(0.4)$ & $4.80(0.5)$ & $5.70(0.7)$ & $5.80(0.8)$ & 0.78 \\
\hline & Placebo & $4.30(0.6)$ & $4.50(0.6)$ & $5.20(0.7)$ & $5.40(0.8)$ & \\
\hline \multirow[t]{2}{*}{ ppp 5-HIAA (nmol//) } & ATD & $28.9(1.6)$ & $15.6(1.0)$ & $30.9(1.1)$ & $16.1(0.8)$ & $<0.001$ \\
\hline & Placebo & $28.9(1.1)$ & $29.4(1.2)$ & $29.8(1.3)$ & $31.6(1.8)$ & \\
\hline \multirow[t]{2}{*}{ ppp 5-HT (nmol/l) } & ATD & $9.80(1.6)$ & $10.7(1.4)$ & $9.20(1.5)$ & $7.90(1.1)$ & 0.25 \\
\hline & Placebo & $10.7(1.7)$ & $10.8(1.7)$ & $9.01(1.2)$ & $11.9(1.5)$ & \\
\hline \multirow[t]{2}{*}{ 5-HT turnover } & ATD & $3.80(0.5)$ & $2.34(0.6)$ & $3.98(0.5)$ & $2.68(0.5)$ & $<0.01$ \\
\hline & Placebo & $3.48(0.5)$ & $3.37(0.6)$ & $3.97(0.5)$ & $3.63\langle 0.6\rangle$ & \\
\hline
\end{tabular}

5-HT, 5-hydroxytryptamine (serotonin); ppp, platelet poor plasma; 5-HIAA, 5-hydroxyindole acetic acid; $\Sigma$ LNAAs, large neutral amino acids (sum of tyrosine, valine, leucine, isoleucine, and phenylalanine)

\section{Discussion}

We have demonstrated for the first time that acute tryptophan depletion (ATD) affects both visceral perception and cognition in d-IBS patients and healthy controls. In addition, we have described an experimental model which can detect changes in the 'brain-gut axis' due to acute alteration of serotonergic activity.

The role of specific $5-\mathrm{HT}$ modulators, mainly $5-\mathrm{HT}_{3}$ antagonists and $5-\mathrm{HT}_{4}$ agonists, in visceral perception has been the subject of earlier studies ${ }^{7,42}$. However, it is not feasible to compare our results with studies using receptor specific 5-HT modulators as both at the CNS as well as the Gl level 5-HT interacts with a number of different 5-HT receptor subclasses that are either stimulatory or inhibitory. It is not known how ATD specifically influences these subreceptors. Data concerning the role of the psychotropic selective serotonin reuptake inhibitors, which enhance the overall availability of $5-\mathrm{HT}$, on visceral perception are conflicting. Reduced oesophageal sensitivity to distension has been described but these drugs seem to lack this effect at the gastric level ${ }^{18}$.

In contrast with most studies evaluating the therapeutic effects of long-term serotonergic modulation on Gl physiology, this study was designed to investigate the effects of acute serotonergic modulation within the brain-gut axis. This is the first time that the effects of lowered 5-HT synthesis using the 
$102 \mid$ Chapter 5

ATD method on both brain and gut function have been studied. ATD significantly lowered the perceptual threshold for first perception but nor for maximal tolerable discomfort. In addition, ATD was associated with increased urge scores specifically in the lower pressure range and overall increased pain scores. The effect of ATD on pain scores was not pressure dependent. As pain is a nociceptive stimulus, our data seem to be in accordance with the results of others who reported that the induced reduction in IBS symptoms by a $5-\mathrm{HT}_{3}$ antagonist was maximal in the absence of visceral nociceptive input ${ }^{43}$. As a possible mechanism explaining these findings it was hypothesized that the inhibitory effect of the $5-\mathrm{HT}_{3}$ antagonist on central neuronal excitability can be overcome by activation of nociceptive rectal afferents. Apart from this biological reason, the lack of a pressure dependent effect for the perception of pain may also be due to a methodological problem. A minority of our subjects reported perception of pain before reaching their threshold for maximal tolerable discomfort. Hence this resulted in large variance of our pain data.

The observed memory impairing effects of ATD on delayed recall are similar to former studies applying ATD in patients with mood disorders as well as in healthy controls ${ }^{21,23,44}$. In addition to impaired memory function, 'mood congruence' is one of the features of the depressive syndrome (that is, depressed patients are more likely to store or recall information that is congruent with their mood state $)^{45}$. The presence of a depression-like memory bias in IBS is however not fully established ${ }^{46,47}$. Whitehead and Palsson suggested that psychological response bias resulting in increased attention and hypervigilance to $\mathrm{Gl}$ sensations is present in IBS and influences visceral perception $^{48}$. In our study, ATD induced a depression-like affective memory bias. We do not know however whether ATD induced increased attention towards $\mathrm{Gl}$ sensations as our word list did not include $\mathrm{Gl}$ annotated words. In future research differentiation between words with and without a $\mathrm{Gl}$ annotation should be made. In our opinion, response bias could potentially have enhanced visceral perception during ATD. This hypothesis integrating cognition in the neurophysiology of visceral perception needs further validation and may have consequences in the clinical practice of IBS. During ATD, we did not find a significant decrease of mood status, using the POMS, in IBS patients or healthy controls. In our opinion however affective memory bias may offer a more objective and sensitive biomarker of central serotonergic dysregulation.

It is difficult to define the primary point of action of ATD on visceral perception. Importantly, 5-HT reuptake by the serotonin reuptake transporter is a mechanism that is relevant in both the digestive tract and $\mathrm{CNS}^{49}$. The fact that ATD lowers central 5-HT synthesis and our findings of ATD induced affective memory bias favour a central influence of $A T D^{20,50}$. In addition, animal studies 
have shown that an acute decrease in serotonergic activity is associated with increased sensory information processing, which is in concordance with enhanced visceral perception during ATD ${ }^{51}$.

Until now, ATD has only been used with regard to changes in CNS functioning. However, our data also strongly suggest that ATD exerts its metabolic action at the level of the gut. Only plasma tryptophan/LNAAs ratios have been routinely assessed as an indication of central serotonergic depletion. We are the first to measure ppp levels of 5-HT and 5-HIAA in addition to plasma tryptophan levels. The significantly decreased plasma 5-HIAA concentrations strongly suggest that 5-HT metabolism in the gut was decreased because the gut is by far the major $(>55 \%)$ contributor to the total $5-$ HIAA plasma poo ${ }^{52}$. Although plasma $5-\mathrm{HT}$ turnover significantly decreased during ATD, this was not reflected by a significant decrease in ppp 5-HT. We hypothesize that blood platelets release $5-\mathrm{HT}$ in order to counterbalance lowered ppp 5-HT concentrations during ATD. The finding that platelet $5-\mathrm{HT}$ was not significantly affected by ATD is in line with evidence that blood platelets act as a $5-\mathrm{HT}$ reservoir and therefore are indicative of long-term changes in 5-HT metabolism.

Apparently, relationships between systemic, GI, and CNS 5-HT metabolism and the influence of acute and chronic serotonergic modulation on each of these systems needs further study. Although it is of interest to know whether pharmacological and metabolic serotonergic modulation primarily acts at the CNS or peripheral level, one has to keep in mind that the brain-gut axis consists of bidirectional neurohumoral pathways and hence modulation at one level may affect various levels of the brain-gut axis ${ }^{10}$.

Increased postprandial ppp 5-HT levels in d-IBS patients and increased numbers of 5-HT containing EC cells in PI-IBS have been observed. This seems paradoxical to our findings of decreased 5-HT levels associated with enhanced visceral perception. Increased postprandial ppp 5-HT levels have been associated with postprandial symptomatology in $d-I B S^{13,16}$. However, we did not show increased ppp 5-HT levels in d-IBS. This can be explained by analytical and methodological differences as well as the fact that the subjects in our study were fasted of any nutrients during the five hours before the assessment of visceral perception and ppp 5-HT. Our results suggest a different, although not significant, effect of ATD on ppp 5-HT levels between patients and controls. This may support evidence concerning disturbed serotonergic metabolism in the $\mathrm{d}$-IBS subgroup type of IBS ${ }^{13,14,16}$. Dunlop et al. showed that both increased EC cells as well as depression are equally important independent predictors of developing PI-IBS ${ }^{5}$. GI motility may be primarily peripherally determined and our study does not solve the contribution 
of central versus peripheral 5-HT in the role of altered motility and perception in IBS. Recently, two tryptophan hydroxylase (rate limiting enzyme of 5-HT synthesis) isoforms have been identified which differentially modulate peripheral and central $5-\mathrm{HT}$ effects ${ }^{53}$. This duality of the $5-\mathrm{HT}$ system may open new avenues for specific investigational and therapeutic approaches exclusively affecting central or peripheral $5-\mathrm{HT}$ actions ${ }^{54}$.

The time window of ATD induced metabolic activity at the CNS level may differ from that at the gut level. However, our cognitive and biochemical results indicated that ATD influenced both the CNS as well as the periphery during our experiments. Our results concern the change in brain-gut interaction due to an acute decrease of serotonergic activity. Whether these findings are also valid in a prolonged change in serotonergic activity by pharmacological or nutritional means needs to be investigated. We did not find a significant effect of ATD on rectal compliance. Although our barostat protocol was primarily designed to detect changes in visceral perception, this suggests that acute changes of serotonergic activity differentially affect visceral perception and rectal compliance. The ATD model mimics characteristics of IBS in healthy controls. During ATD, healthy controls had urge scores comparable with those of the patients in the placebo condition. The fact that ATD did not increase pain scores in the control group to levels observed in the patient group suggest that pain and urge perception are different entities.

Our results support further study on the effect of serotonergic modulation on the brain-gut interaction in IBS. We have provided evidence that serotonergic modulation by ATD affects both visceral perception as well as cognition in d-IBS patients and controls. Simultaneous measurement of brain and gut function and the application of ATD contribute to elucidation of the complex pathophysiology of IBS. 


\section{References}

1. Talley NJ, Spiller R. Irritable bowel syndrome: a little understood organic bowel disease? Lancet 2002;360:555-564.

2. Mayer EA, Gebhart GF. Basic and clinical aspects of visceral hyperalgesia. Gastroenterology 1994;107:271-293.

3. Walker EA, Roy-Byrne PP, Katon WJ, Li L, Amos D, Jiranek G. Psychiatric illness and irritable bowel syndrome: a comparison with inflammatory bowel disease. Am J Psychiatry 1990;147:1656-1661.

4. Delvaux M. Role of visceral sensitivity in the pathophysiology of irritable bowel syndrome. Gut 2002;51 Suppl 1:i67-71.

5. Dunlop SP, Jenkins D, Neal KR, Spiller RC. Relative importance of enterochromaffin cell hyperplasia, anxiety, and depression in postinfectious IBS. Gastroenterology 2003;125: 16511659.

6. Charney DS. Monoamine dysfunction and the pathophysiology and treatment of depression. $\mathrm{J}$ Clin Psychiatry 1998:59 Suppl 14:11-14.

7. Camilleri M. Serotonergic modulation of visceral sensation: lower gut. Gut 2002;51 Suppl 1: i81-86.

8. Creed F, Fernandes L, Guthrie E, Palmer S, Ratcliffe J, Read N, Rigby C, Thompson D, Tomenson B. The cost-effectiveness of psychotherapy and paroxetine for severe irritable bowel syndrome. Gastroenterology 2003;124:303-317.

9. Jackson JL, O'Malley PG, Tomkins $G$, Balden $E$, Santoro J, Kroenke K. Treatment of functional gastrointestinal disorders with antidepressant medications: a meta-analysis. Am J Med 2000;108:65-72.

10. Kilkens TO, Honig A, Rozendaal N, Van Nieuwenhoven MA, Brummer RJ. Serotonergic modulators in the treatment of irritable bowel syndrome - influence on psychiatric and gastrointestinal symptoms. Aliment Pharmacol Ther 2003;17:43-51.

11. Clouse RE. Antidepressants for irritable bowel syndrome. Gut 2003;52:598-599.

12. Kim DY, Camilleri M. Serotonin: a mediator of the brain-gut connection. Am J Gastroenterol 2000;95:2698-2709

13. Houghton LA, Atkinson W, Whitaker RP, Whorwell PJ, Rimmer MJ. Increased platelet depleted plasma 5-hydroxytryptamine concentration following meal ingestion in symptomatic female subjects with diarrhoea predominant irritable bowel syndrome. Gut 2003;52:663-670.

14. Singh RK, Pandey HP, Singh RH. Correlation of serotonin and monoamine oxidase levels with anxiety level in diarrhea-predominant irritable bowel syndrome. Indian J Gastroenterol 2003;22:88-90.

15. Camilleri M, Northcutt AR, Kong S, Dukes GE, McSorley D, Mangel AW. Efficacy and safety of alosetron in women with irritable bowel syndrome: a randomised, placebo-controlled trial. Lancet 2000;355:1035-1040.

16. Bearcroft CP, Perrett D, Farthing MJ. Postprandial plasma 5-hydroxytryptamine in diarrhoea predominant irritable bowel syndrome: a pilot study. Gut 1998;42:42-46.

17. Sanger GJ, Yoshida M, Yahyah M, Kitazumi K. Increased defecation during stress or after 5hydroxytryptophan: selective inhibition by the $5-\mathrm{HT}(4)$ receptor antagonist, SB-207266. Br J Pharmacol 2000;130:706-712.

18. Tack J, Sarnelli G. Serotonergic modulation of visceral sensation: upper gastrointestinal tract. Gut 2002;51 Suppl 1:i77-80.

19. Moore P, Landolt H-P, Seifritz E, Clark C, Batthi T, Kelsoe J, Rapaport M, Gillin JC. Clinical and Physiological Consequences of Rapid Tryptophan Depletion. Neuropsychopharmacology 2000;23:601-622.

20. Carpenter LL, Anderson GM, Pelton GH, Gudin JA, Kirwin PD, Price LH, Heninger GR, McDougle CJ. Tryptophan depletion during continuous CSF sampling in healthy human subjects. Neuropsychopharmacology 1998;19:26-35. 
$106 \mid$ Chapter 5

21. Sobczak S, Riedel WJ, Booij I, Aan Het Rot M, Deutz NE, Honig A. Cognition following acute tryptophan depletion: difference between first-degree relatives of bipolar disorder patients and matched healthy control volunteers. Psychol Med 2002;32:503-515.

22. Klaassen T, Riedel WJ, Deutz NE, Van Praag HM. Mood congruent memory bias induced by tryptophan depletion. Psychol Med 2002;32:167-172.

23. Riedel WJ, Klaassen T, Deutz NE, van Someren A, van Praag HM. Tryptophan depletion in normal volunteers produces selective impairment in memory consolidation. Psychopharmacology (Berl) 1999;141:362-369.

24. Yatham LN, Liddle PF, Shiah IS, Lam RW, Adam MJ, Zis AP, Ruth TJ. Effects of rapid tryptophan depletion on brain 5-HT(2) receptors: a PET study. Br J Psychiatry 2001;178:448453.

25. Van Nieuwenhoven MA, Valks SDM, Sobczak S, Riedel WJ, Brummer R-JM. Acute tryptophan depletion slows gastric emptying in females. British Journal of Nutrition 2004;91:16.

26. Sheehan D. MINI International neuropsychiatric Interview: Tampa: University of South Florida; 1994

27. Hamilton $M$. Development of a rating scale for primary depressive illness. $\mathrm{Br} J \mathrm{Soc}$ Clin Psychol 1967;6:278-296.

28. Arrindell WA, Ettema JHM. SCL-90. Een multidimensionale psychopathologie indicator [SCL90. A multidimensional indicator of psychopathology]. In. Lisse, The Netherlands: Swets \& Zeitlinger; 1986.

29. Mykletun A, Stordal E, Dahl AA. Hospital Anxiety and Depression (HAD) scale: factor structure, item analyses and internal consistency in a large population. $\mathrm{Br} J$ Psychiatry 2001;179:540-544

30. Luteijn F, van der Ploeg FAE. Handleiding Groninger Intelligentie test (GIT) [Manual Groninger Intelligence Test]. Lisse, the Netherlands: Swets and Zeitlinger; 1983.

31. Drossman DA, Heaton $K$, Irvine EJ, Muller-Lissner S. Functional bowel disorders and functional abdominal pain. In: Drossman DA, Corazziari E, Talley N, Thompson WG, Whitehead WE, editors. The functional gastrointestinal disorders. Diagnosis, pathophysiology and treatment: A multinational consensus. McLean, VA: Degnon Associates;2000:351-398.

32. Ledochowski $M$, Sperner-Unterweger $B$, Fuchs $D$. Lactose malabsorption is associated with early signs of mental depression in females: a preliminary report. Dig Dis Sci 1998;43: 2513-2517.

33. Houghton LA, Lea $R$, Jackson $N$, Whorwell PJ. The menstrual cycle affects rectal sensitivity in patients with irritable bowel syndrome but not healthy volunteers. Gut 2002;50:471-474.

34. Maes $M$, Scharpe $S$, Verkerk $R, D$ Hondt $P$, Peeters $D$, Cosyns $P$, Thompson $P$, De Meyer $F$, Wauters $A$, Neels $H$. Seasonal variation in plasma L-tryptophan availability in healthy volunteers. Relationships to violent suicide occurrence. Arch Gen Psychiatry 1995;52: 937-946.

35. Sloots CE, Felt-Bersma RJ, Cuesta MA, Meuwissen SG. Rectal visceral sensitivity in healthy volunteers: influences of gender, age and methods. Neurogastroenterol Motil 2000;12: 361-368.

36. Menair DM, Lorr M, Droppleman LF. Manual for the Profile of Mood States (POMS) manual. Educational and Industrial Testing Service. San Diego, CA; 1992.

37. Bell $C$, Abrams J, Nutt $D$. Tryptophan depletion and its implications for psychiatry. $\mathrm{Br} J$ Psychiatry 2001;178:399-405.

38. Maes $M$, Wauters A, Verkerk R, Demedts P, Neels $H$, Van Gastel A, Cosyns $P$, Scharpe $S$, Desnyder R. Lower serum L-tryptophan availability in depression as a marker of a more generalized disorder in protein metabolism. Neuropsychopharmacology 1996;15:243-251.

39. Turnell DC, Cooper JD. Rapid assay for amino acids in serum or urine by pre-column derivatization and reversed-phase liquid chromatography. Clin Chem 1982;28:527-531.

40. Stronks DL, Tulen JH, Verheij R, Boomsma F, Fekkes D, Pepplinkhuizen L, Mantel GW, Passchier J. Serotonergic, catecholaminergic, and cardiovascular reactions to mental stress in female migraine patients. A controlled study. Headache 1998;38:270-280. 
41. Fekkes D, Timmerman L, Pepplinkhuizen L. Effects of clomipramine on plasma amino acids and serotonergic parameters in panic disorder and depression. Eur Neuropsychopharmacol 1997;7:235-239.

42. Talley NJ. Review article: 5-hydroxytryptamine agonists and antagonists in the modulation of gastrointestinal motility and sensation: clinical implications. Aliment Pharmacol Ther 1992;6: 273-289.

43. Berman SM, Chang L, Suyenobu B, Derbyshire SW, Stains J, Fitzgerald L, Mandelkern M, Hamm L, Vogt B, Naliboff BD, Mayer EA. Condition-specific deactivation of brain regions by 5-HT3 receptor antagonist Alosetron. Gastroenterology 2002;123:969-977.

44. Park SB, Coull JT, McShane RH, Young AH, Sahakian BJ, Robbins TW, Cowen PJ. Tryptophan depletion in normal volunteers produces selective impairments in learning and memory. Neuropharmacology 1994;33:575-588.

45. Elliott R, Rubinsztein JS, Sahakian BJ, Dolan RJ. The neural basis of mood-congruent processing biases in depression. Arch Gen Psychiatry 2002;59:597-604.

46. Toner BB, Garfinkel PE, Jeejeebhoy KN, Scher H, Shulhan D, Di Gasbarro I. Self-schema in irritable bowel syndrome and depression. Psychosom Med 1990;52:149-155.

47. Gomborone JE, Dewsnap PA, Libby GW. Farthing MJ. Selective affective biasing in recognition memory in the irritable bowel syndrome. Gut 1993;34:1230-1233.

48. Whitehead WE, Palsson OS. Is rectal pain sensitivity a biological marker for irritable bowel syndrome: psychological influences on pain perception. Gastroenterology 1998;115: 1263-1271.

49. Wade PR, Chen J, Jaffe B, Kassem IS, Blakely RD, Gershon MD. Localization and function of a 5-HT transporter in crypt epithelia of the gastrointestinal tract. J Neurosci 1996;16: 2352-2364.

50. Nishizawa S, Benkelfat C, Young SN, Leyton M, Mzengeza S, de Montigny C, Blier P, Diksic $M$. Differences between males and females in rates of serotonin synthesis in human brain. Proc Natl Acad Sci U S A 1997;94:5308-5313.

51. Jacobs $B L$, Fornal $C A$. Activity of serotonergic neurons in behaving animals. Neuropsychopharmacology 1999;21:9S-15S.

52. Lambert GW, Kaye DM, Cox HS, Vaz M, Turner AG, Jennings GL, Esler MD. Regional 5hydroxyindoleacetic acid production in humans. Life Sci 1995;57:255-267.

53. Walther DJ, Bader M. A unique central tryptophan hydroxylase isoform. Biochem Pharmacol 2003;66:1673-1680.

54. Walther DJ, Peter JU, Bashammakh S, Hortnagl H, Voits M, Fink H, Bader M. Synthesis of serotonin by a second tryptophan hydroxylase isoform. Science 2003;299:76. 


\section{Chapter 6}

Acute tryptophan depletion alters cortical activation following visceral stimuli

MA van Nieuwenhoven, TO Kilkens, EA Evers, RJ Brummer, WA Backes Submitted 


\section{Abstract}

\section{Background}

Serotonin is involved in visceral perception. Acute tryptophan depletion (ATD) is an established method to lower the serotonergic activity in the brain.

Aim

The aim of this study was to investigate the effect of ATD on cortical activation during painful and non-painful rectal distension.

\section{Methods}

Twelve healthy females (19-25 year) were studied under both a control condition and during ATD.

Functional magnetic resonance imaging ( $(\mathrm{MMR}$ ) sessions were performed on a $1.5 \mathrm{~T}$ system using block-related rectal barostat stimuli, consisting of non-painful and painful distensions in random order, interleaved with 30 seconds rest intervals.

Resuits

Significant differences in cortical activation as a result of ATD were found for painful distension (high > rest $x$ ATD contrast). ATD induced more activation in the left anterior cingulate cortex $(\mathrm{ACC}$ ), the left superior frontal cortex and right insula and putamen. Non-painful distension (low > rest $X$ ATD contrast) resulted in more activation of the left putamen as a result of ATD.

Painful rectal distension (high > rest contrast) resulted in activation of the right insula and the right inferior frontal cortex, whereas non-painful distension (low > rest contrast) resulted in activation of the right insula, the right $A C C$ and the right inferior and left orbitofrontal cortex.

\section{Conclusions}

ATD leads to a shift from primary visceral pain sensation as represented by activation of the right insula and the inferior frontal operculum towards a more affective or emotional experience of the visceral pain sensation, as represented by increased activation of the ACC, superior frontal cortex and right insula. 
Tryptophan depletion, rectal stimuli and IMRI $\mid 111$

\section{Introduction}

The pathophysiology of irritable bowel syndrome (IBS) is not completely understood. A multicomponent conceptual model of IBS has been postulated involving physiological, affective, cognitive and behavioural factors ${ }^{1}$. The "braingut axis' is a theoretical model describing the bidirectional neural pathways linking cognitive and emotional centres in the brain to neuroendocrine centres, the enteric nervous system and the immune system and plays a major role in the concept of IBS ${ }^{2}$. IBS is associated with visceral hypersensitivity and with a high co-occurrence of psychiatric symptoms, in particular affective dysregulation ${ }^{3}$.

Serotonin (5-HT) is a biogenic amine that functions as a neurotransmitter and is located predominantly in the gastrointestinal (GI) tract $(80 \%$ of body $5-\mathrm{HT})$. About $5 \%$ is located centrally in the brain and cerebrospinal fluid. 5 -HT is peripherally involved in the regulation of $\mathrm{Gl}$ secretion, motility and perception, whereas in the central nervous system (CNS) it plays a pivotal role in the regulation of mood and cognition ${ }^{4}$. Hence, $5-\mathrm{HT}$ is a key denominator of the 'brain-gut axis',5,

Functional magnetic resonance imaging (fMRI) is now widely used to study cognitive, motor and sensory systems in the brain ${ }^{7}$. The visualization of the central response to visceral sensation and the possibility to study abnormalities of the brain-gut axis in IBS and other functional gastrointestinal disorders has gained wide interest, as well as the effect of pharmacological intervention on the brain-gut axis ${ }^{8-29}$. Recently, Derbyshire ${ }^{13}$ performed a systematic review to establish which brain structures are involved in visceral perception. The most consistently activated structure appeared to be the insula. Additional regions comprised the anterior cingulate cortex (ACC), the prefrontal cortex, and the primary sensory cortex (S1). Although results are conflicting there appear to be differences between IBS patients and control subjects regarding the central processing of lower $\mathrm{Gl}$ tract distension.

Acute tryptophan depletion (ATD) is a method to temporarily reduce 5-HT synthesis by decreasing the availability of its precursor tryptophan. This can be accomplished by administration of an amino acid mixture lacking tryptophan ${ }^{30}$. Following ATD a substantial reduction of brain serotonin synthesis as well as decreased levels of tryptophan have been demonstrated in humans ${ }^{31-33}$.

ATD has been used to investigate the role of the central-5-HT system in patients with affective disorders (depression and anxiety) and their first-degree relatives ${ }^{34,35}$. It serves as a biological model for inducing symptoms of depression ${ }^{36}$. In gastroenterological research ATD has been shown to delay gastric emptying $^{37}$.

Recently, we demonstrated that ATD can alter brain-gut responses, i.e. ATD enhanced visceral pain perception of rectal balloon distensions and induced an 
$112 \mid$ Chapter 9

affective memory bias towards a preferential loss of positive material in IBS patients as well as in healthy controls ${ }^{38}$. As ATD has been associated with enhanced visceral perception we hypothesised that ATD induces changes in the central response to visceral stimulation.

Therefore, the aim of the current study is to investigate the influence of a reduced serotonergic synthesis, by means of ATD, on cortical activation to visceral stimulation in healthy female subjects.

\section{Materials and methods}

\section{Subjects}

Twelve right-handed healthy females (19-25 y) were included. To avoid possible effects of the menstrual cycle, the subjects who did not use oral contraceptives were studied during the follicular phase of the menstrual cycle. Female subjects were studied because the rate of central 5-HT synthesis is lower in females than in males ${ }^{31}$. Hence, they are more sensitive to ATD.

Exclusion criteria comprised abdominal surgery (other than appendectomy and cholecystectomy), use of medications other than oral contraceptives within fourteen days prior to testing, lactose intolerance ${ }^{39}$, a positive first-degree psychiatric family history ${ }^{40}$, any history of psychiatric disease or use of psychoactive medication, or Hospital Anxiety and Depression Scale (HADS) scores equal to or above eight. The Medical Ethics Committee of the University Hospital Maastricht approved the study protocol and all subjects gave their informed written consent before start of the study.

\section{Study design}

After inclusion, but at least one day before the start of the fMRl-barostat protocol the pressures inducing minor urge to defecate (non-painful stimulus) and the maximal tolerable pressure (painful stimulus) were obtained in each individual using a rectal barostat semirandom distension protocol ${ }^{38}$.

The fMRI-barostat protocol comprised the measurement of cortical response patterns to rectal non-painful and painful stimuli in a placebo-controlled doubleblind randomised crossover design under a placebo condition and an ATD condition, respectively. The two test days were separated by at least one week. During the test days subjects ingested the amino acid mixture at $t=0$ hour. At $t=4$ hour the fMRI-barostat experiment was started. 
Tryptophan depletion, rectal stimul and $\mathrm{MMR} \mid 113$

\section{Tryptophan depletion}

The amino acid mixtures were prepared by the department of Pharmacy of the University Hospital. The ATD mixture consisted of 15 amino acids including five large neutral amino acids (LNAAs). It contained no tryptophan. The placebo mixture contained the same amino acids plus 3 grams of tryptophan ${ }^{40}$. After an overnight fast, the subjects consumed the amino acid mixtures dissolved in $250 \mathrm{ml}$ water as quickly as possible, using a nose clamp to avoid the unpleasant smell of the mixtures. Water, decaffeinated tea and peppermint were available to neutralize the unpleasant taste of the mixtures.

\section{Amino acids}

Tryptophan and the ratio tryptophan/ $\mathrm{LNAA}$ (sum of tyrosine, leucine, phenylalanine, isoleucine, valine) were measured at $t<0$ and $t=4$ hour, using HPLC ${ }^{41}$. Differences between ATD and placebo and between baseline and 4 hour after amino acid ingestion were compared using a paired sample $t$-test. $P$-values of $\leq 0.05$ were considered statistically significant.

\section{Visceral perception}

The bowel preparation consisted of a water enema administered one hour before the barostat procedure. The rectal probe was lubricated and placed into the rectum with the attached end of the bag at $3 \mathrm{~cm}$ from the external anal sphincter. The probe consisted of a $700 \mathrm{ml}$ polyethylene bag secured on a rectal catheter (external diameter $=18$ French). The catheter was connected to the electronic distension device (Electronic Barostat; Distender Series II; G \& J Electronics Inc., Toronto, Ontario, Canada) using the Protocol Plus Deluxe Software ( $G$ \& J Electronics $I n c$.). The barostat inflation rate was $50 \mathrm{ml} / \mathrm{s}$ to a constant pressure plateau. Subjects were not given any auditory or visual clues that they were to receive a stimulus and were not told the exact nature of the distension protocol.

Determination of individual low and high distension pressures: In order to unfold the probe a 5 -minute $10-\mathrm{mmHg}$ distension, followed by a 1-minute $20-\mathrm{mmHg}$ distension was administered, respectively. Subsequently, the barostat protocol consisted of intermittent semi-random staircase distensions of 60 -second duration $(15,10,25,20 \mathrm{mmHg}$ etcetera) separated by an interval of 30 -second baseline pressure ${ }^{42}$. The end point to stop the series of distensions was the perceptual threshold for maximal tolerable pressure. During each distension (after thirteen seconds of distension) subjects were asked to report their perception level on a panel (Deluxe Perception Panel; G\&J Electronics Inc.) with six levels labelled: 1, not perceptible; 2, first perception; 3, minor urge; 4 , normal urge; 5 , strong urge and 6 , maximal tolerable pressure. The 
level 3 and the level 6 distension pressures constituted the low (minor urge $=$ non-painful) and high (maximal tolerable pressure=painful) distension pressures, respectively. These low and high distension pressures were subsequently used during the fMRI-barostat experiments.

\section{fMRI - barostat protocol}

Images were acquired on a 1.5 T MR scanner (Philips ACS-NT, Philips Medical Systems), which was equipped with a standard receiver head coil. Head fixation was achieved by two foam cushions and by a tape across the subject's head. The scanner room was dimly lit and the subjects were instructed to close their eyes during the experiment.

On the basis of $\mathrm{T}_{1}$-weighted survey scans in three orthogonal directions, an approximately $12 \mathrm{~cm}$ thick stack of slices was defined, encompassing transversal slices that covered the entire cerebrum and as much as possible of the cerebellum.

The functional scan session consisted of a single shot multiple slice $\mathrm{T}_{2}{ }^{*}$ sensitive echo planar imaging (EPI) sequence, with the following parameters: repetition time $3715 \mathrm{~ms}$, echo time $40 \mathrm{~ms}$, flip angle $90^{\circ}$, matrix dimension $64 \times 64,34$ contiguous slices and a voxel size of $3.5 \times 3.5 \times 3.5 \mathrm{~mm}$. The fMRI signal is based on the blood oxygen level dependent (BOLD) effect. Before the acquisition of functional images started, two dummy full brain scans were acquired to run the system in a steady state in order to avoid switch-on effects. The total acquisition time was 12.5 minutes for the fMRI examination.

Rectal painful and non-painful distensions were applied by a barostat, which was placed outside the MR room, by means of long tubes (10 meter) which were guided through the wave guide of the MRI room wall. The distensions were presented in a block design: epochs of eight scans without rectal distension were followed by eight scans with rectal distension. This sequence was repeated up to the total of 202 scans. The rectal barostat stimulation/distension paradigm consisted of six non-painful distensions and six painful distensions, respectively, in random order, each distension lasted 30 seconds and were interleaved with 30 seconds rest $(0 \mathrm{mmHg})$ intervals (rest distension).

For anatomical reference, a 3D $T_{1}$-weighted fast-field echo scan succeeded the functional scans with the following parameters: repetition time $11 \mathrm{~ms}$, echo time $38 \mathrm{~ms}$, flip angle $8^{\circ}$, matrix dimensions $256 \times 256,128$ contiguous slices and a voxel size of $1 \times 1 \times 1 \mathrm{~mm}$.

Image analysis

The image data were analysed using the Statistical Parametric Mapping (SPM99) software package implemented in MATLAB (The Mathworks, Inc.) 
Tryptophan depletion, rectal stimuli and FMRI $\mid 115$

and run on a Windows PC. The scans were first corrected for small motion artefacts by $3 \mathrm{D}$ rigid-body realignment to the first volume scan of each timeseries. Subsequently, the images were spatially normalised by transformation into standard space, using the EPI template image of the MNI (Montreal Neurological Institute) as a reference to the stereotaxic atlas of Talairach and Tournoux ${ }^{43}$. The voxel sizes of the normalized images were $2 \times 2 \times 2 \mathrm{~mm}$. The stereotaxic coordinates of the Montreal Neurological Institute were used to report the location of the observed activation foci. To enhance the spatial signal-to-noise ratio and to facilitate inter-subject averaging, the normalised images were smoothed with a Gaussian filter with a full-width at half maximum of $8 \mathrm{~mm}$.

For the group analysis the SPM design matrix considered the global activity as a confound which was accounted for by global scaling. Temporal smoothing, a low-pass filter $(1 / 120 \mathrm{~Hz})$ and delaying of the modelled response function by six seconds were applied to enhance that part of the haemodynamic response that correlated with stimulus sequence. The resulting set of images represents statistical parametric maps of the $t$ statistic SPM $\{t\}$. The statistical inferences about the deflecting fMRI signal were based on the theory of random Gaussian fields. The $t$-maps were transformed to the unit normal probability distribution to allow inferences based on Gaussian $p$-values and Z-values. Subsequently, statistical random-effect group inferences were made on the differences in cortical activation as a result of ATD between the rest and low distension pressures stimulus (low > rest $X$ ATD contrast) and between the rest and the high distension pressures stimulus (high > rest $X$ ATD contrast). The effect of both low distension pressures (low $>$ rest contrast) and high distension pressures (high > rest contrast) on cortical activation was measured as well in the placebo experiment. If necessary, a small volume correction was performed using $10 \mathrm{~mm}$ spheres, based on the a prioriprediction that activation will occur in the following regions of interests (ROI): insula, ACC, prefrontal cortex, orbitofrontal cortex, thalamus, primary somatosensory cortex, motor cortex and amygdala ${ }^{13}$. In addition, we assessed the main effect of ATD on the overall activation compared to the placebo condition by comparing the non-stimulus conditions $(0 \mathrm{mmHg})$ in both experiments as a control for non-specific ATD effects. MRIcro (http://web.arizona.edu/ cnl/ mricro.htm) was used to label the coordinates and Brodmann areas of the anatomical regions. 
$116 \mid$ Chapter 6

\section{Results}

\section{Subject characteristics}

Subject characteristics including mean non-painful and painful distension pressures are listed in Table 6.1.

Table 6.1 Subject characteristics (mean (SE)).

\begin{tabular}{lc}
\hline & $n=12$ \\
\hline Females $(n)$ & 12 \\
Use of oral contraceptives $(n)$ & 9 \\
Age $(y)$ & $22.7(0.5)$ \\
HADS score (total) & $2.67(0.6)$ \\
Non-painful distension pressure $(\mathrm{mmHg})$ & $19.6(1.9)$ \\
Painful distension pressure $(\mathrm{mmHg})$ & $52.1(3.5)$ \\
\hline
\end{tabular}

HADS, Hospital Anxiety and Depression Scale.

\section{Amino acids}

No significant baseline differences between ATD and the placebo were found for both the plasma tryptophan levels and the ratio tryptophan $/ \Sigma L N A A ' s, p=0.21$ and $p=0.17$, respectively. The analysis of amino acid levels showed that our depletion was successful. During ATD the plasma tryptophan $(-58 \%)$ and the ratio tryptophan $/ \Sigma$ LNAA's $(-85 \%)$ decreased significantly, compared to the baseline values, $p<0.001$ and $p<0.001$, respectively. For values see Table 6.2.

Table 6.2 Biochemical parameters at $t<0$ and $t=4$ hour (mean (SE)).

\begin{tabular}{llll}
\hline & & $t<0 h$ & $t=4 h$ \\
\hline Tryptophan $(\mu \mathrm{mol} / \mathrm{l})$ & ATD & $34.4(1.3)$ & $14.4(1.1)$ \\
& placebo & $37.3(1.8)$ & $74.6(6.8)$ \\
Tryptophan $/$ LLNAAs & ATD & $0.15(0.02)$ & $0.02(0.01)$ \\
& placebo & $0.16(0.05)$ & $0.13(0.01)$ \\
\hline
\end{tabular}

LNAAs, Large neutral amino acids.

\section{fMRI}

Painful rectal distension (high > rest contrast) resulted in activation of the right insula and the inferior frontal cortex, whereas non-painful distension (low $>$ rest contrast) resulted in activation of the right insula, the right $A C C$ and the inferior and orbitofrontal cortex. Small volume correction was required. (Figures 6.1c, 6.1d and Table 6.3). No significant differences could be observed between the ATD and placebo conditions during the 30 seconds rest intervals. 
Significant differences in cortical activation as a result of ATD were found for painful distension (high > rest $x$ ATD contrast). ATD induced more activation in the left $A C C$, the left superior frontal cortex and right insula and putamen (Figures $6.1 \mathrm{a}$ and Table 6.3). The non-painful distension (low $>$ rest $x$ ATD contrast) resulted in more activation in the left putamen an insula as a result of ATD. Small volume correction was required (See Figure 6.1b and Table 6.3).

A
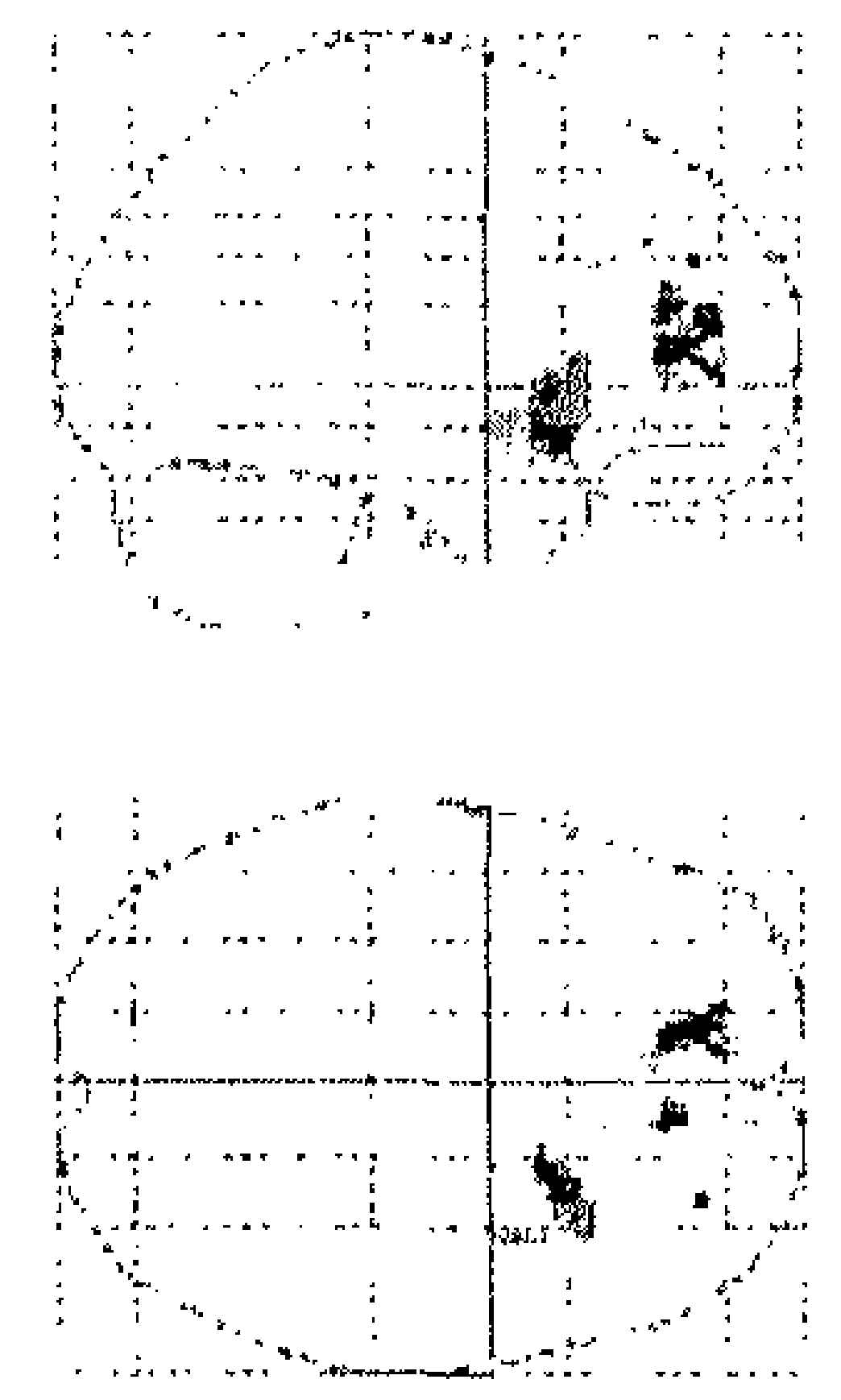

C
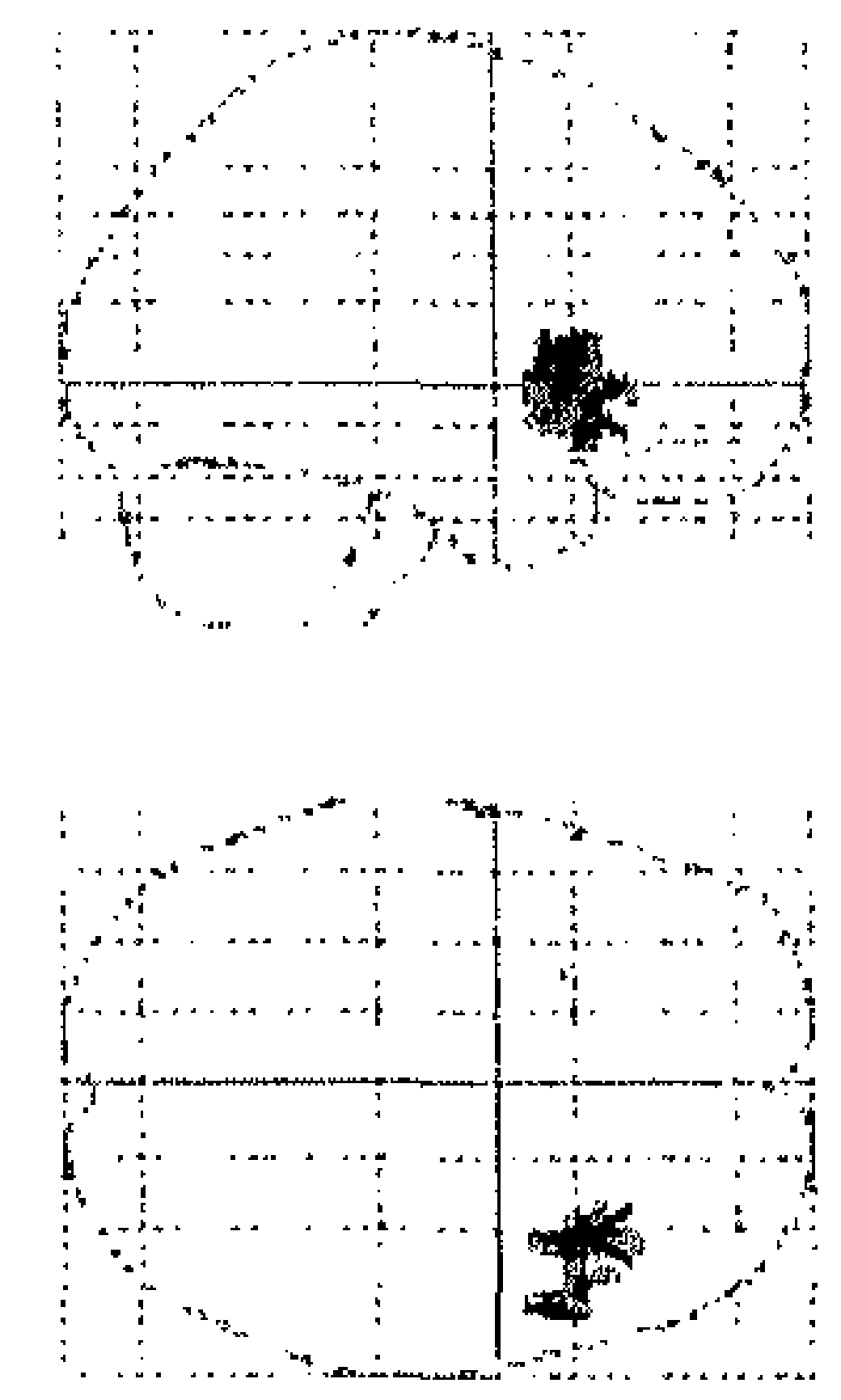

$\mathrm{B}$
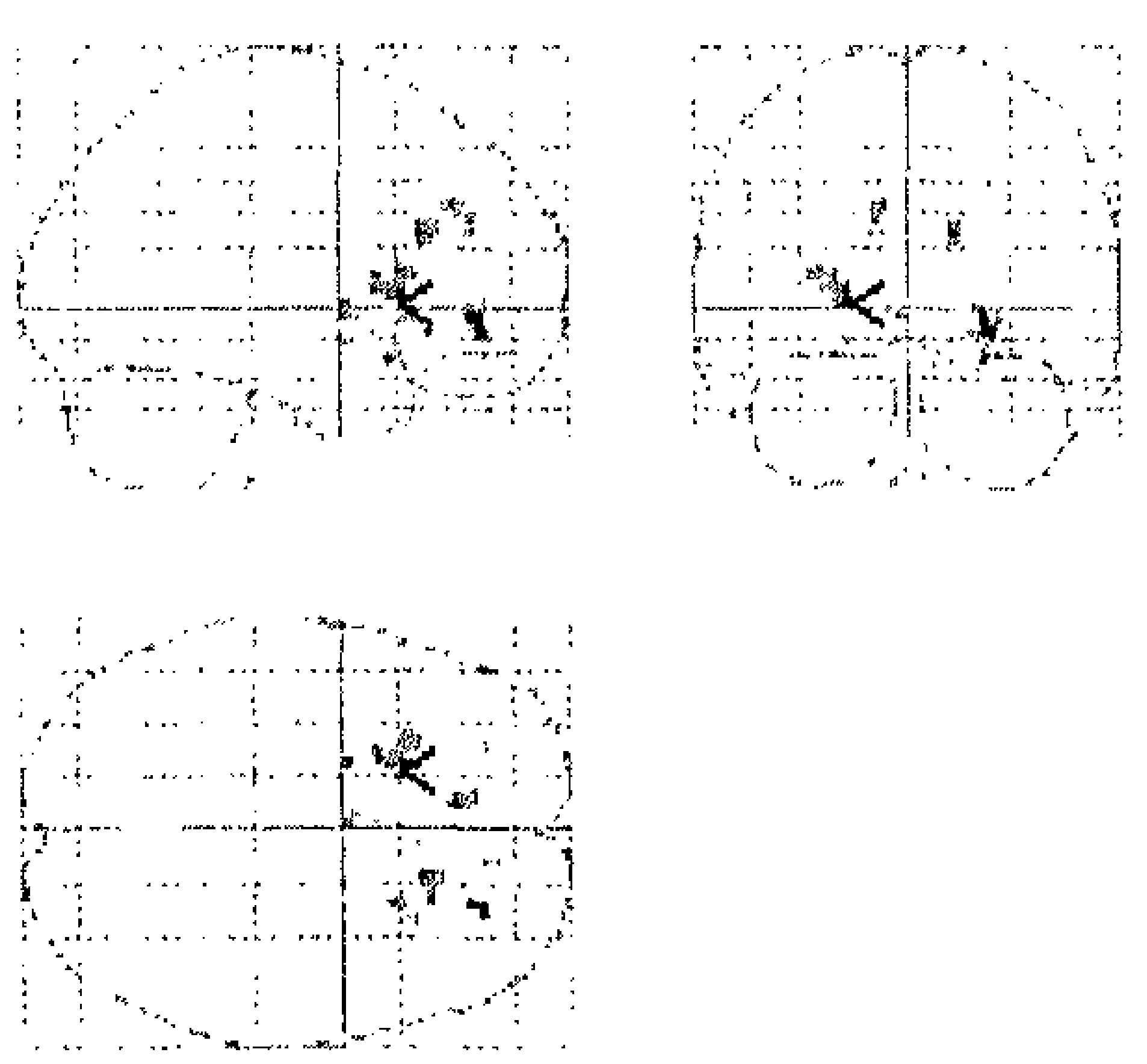

D
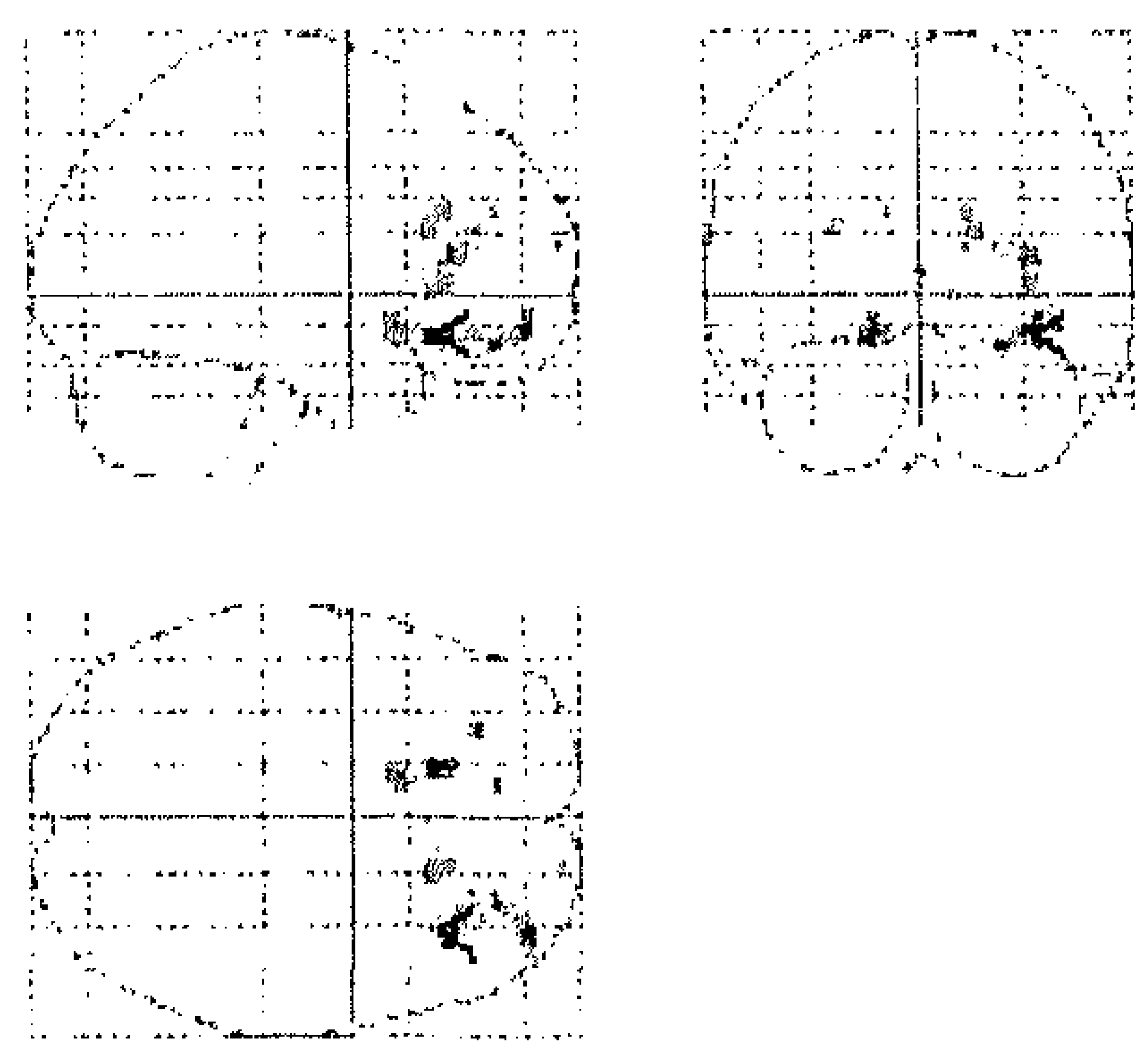

Figure 6.1 Group mean activations for the (1a) "high > rest $x$ ATD contrast", the (1b) "low > rest $x$ ATD contrast", the (ic) "high > rest contrast" and the (1d) "low > rest contrast". For details see text and Table 6.3. 
$118 \mid$ Chapter 6

Table 6.3 Significant effect of ATD on BOLD response to rectal stimuli in painful (high>rest $x$ ATD contrast) and non-painful stimuli (low>rest $x$ ATD contrast). The effects of painful and non-painful rectal stimuli on BOLD response are demonstrated by (highrrest contrast) and (lowrrest contrast). Stereotactic coordinates ( $x$, mediolateral; $y$, rostrocaudal; $z$, dorso-ventral) are presented according to the Montreal Neurological Institute coordinate system.

\begin{tabular}{lcccc}
\hline Increased activation & T-values & $\mathrm{p}$ & $x y z$ & BA \\
\hline High $>$ rest $x$ ATD contrast & & & & \\
$\quad$ Left ACC & 6.78 & 0.02 & $-12,42,8$ & 32 \\
& 5.79 & 0.02 & $-14,50,16$ & 32 \\
Left superior frontal & 4.39 & 0.02 & $-10,54,6$ & 10 \\
Right insula & 6.08 & $<0.01$ & $20,12,-10$ & $11 / 48$ \\
& 5.83 & $<0.01$ & $26,16,-14$ & 48 \\
Right putamen & 5.83 & $<0.01$ & $22,14,-2$ & \\
Low $>$ rest $x$ ATD contrast & & & & \\
Left insula & 4.13 & 0.016 & $-30,20,12$ & 48 \\
& & & & \\
High > rest contrast & & & & 48 \\
Right inferior frontal operculum & 7.33 & $<0.01$ & $52,12,8$ & 48 \\
Right insula & 6.82 & $<0.01$ & $36,16,10$ & $47 / 48$ \\
& 6.63 & $<0.01$ & $36,20,-12$ & \\
Low $>$ rest contrast & & & & 11 \\
Left sup orbitofrontal & 7.02 & $<0.01$ & $-16,26,-14$ & 47 \\
Right mid superior orbitofrontal & 5.73 & $<0.01$ & $36,56,-10$ & $47 / 48$ \\
Right insula & 4.31 & $<0.01$ & $34,28,4$ & 32 \\
Right ACC & 4.36 & $<0.01$ & $14,30,26$ & \\
\hline
\end{tabular}

$B A$, Brodmann area; $A C C$, anterior cingulate cortex; ATD, acute tryptophan depletion.

\section{Discussion}

Our findings demonstrate that a painful rectal distension leads to a BOLD response of the right insula and inferior frontal operculum. ATD leads to an increased activation in the left ACC, the left superior frontal cortex and right insula and putamen. Non-painful distension leads to a BOLD response in the right insula, right ACC and the left and right superior orbitofrontal cortex. ATD leads to significant more activation of the left insula.

Our data confirm the earlier reported activation of the frontal, insular and ACC regions in response to visceral stimulation ${ }^{13}$. Studies comparing cortical response patterns in IBS patients with healthy controls show inconsistent results. Both increased ${ }^{19,24,44}$ and decreased ${ }^{8,23}$ cortical activity in patients with IBS have been reported. Pharmacological intervention in IBS patients with a $5-\mathrm{HT}_{3}$ receptor antagonist, which reduces symptoms in female IBS patients, showed a reduced cerebral activation in emotional motor system regions, which are rich in $5-\mathrm{HT}_{3}$ receptors, but no significant change in areas activated by pain $^{10,12}$. 
We previously demonstrated that ATD enhanced visceral pain perception of rectal balloon distensions ${ }^{38}$. The present study demonstrates that ATD also leads to a shift in BOLD response towards the left ACC, the left superior frontal cortex and the right insula and putamen, following a painful rectal stimulus.

The insula is believed to mediate primarily visceral sensations (taste, smell, gastric, colonic, and other visceral inputs) including rectal stimuli. The insular cortex neurons distributed between the taste area and the visceral area receive convergent inputs from baroreceptor, chemoreceptor, gustatory and nociceptive organs and may have roles in taste aversion or in the regulation of visceral responses. Recently it has been suggested that the insula represents one main structure where the affective-motivational component of the unpleasantness of tonic pain perception is encoded ${ }^{45}$.

The ACC is thought to mediate the affective or "emotional" content of sensory information. It is also involved in transient mood changes, depression and anxiety disorders and autonomic regulation ${ }^{46-48}$.

The orbital cortex receives several sensory inputs (olfactory, gustatory, visceral afferent, somatic sensory, and visual) that appear to be related to feeding. It also receives many limbic inputs and may therefore serve as a substrate to integrate viscerosensory information with affective signals ${ }^{49}$.

Recently, it has been hypothesized that behaviourally relevant nociceptive information without additional information from the tactile system is represented in the putamen and made available for pain related motor responses ${ }^{50}$. The frontal operculum has been shown to participate in the processing of somatosensory stimuli ${ }^{51}$.

A unique characteristic of gastrointestinal sensation is that it evokes stronger emotional reactions compared to a perceived intensity matched somatic stimulus $^{52}$. Recently, the effect of negative emotional context on the brain processing of gastrointestinal sensation has been examined ${ }^{53}$. The study revealed that oesophageal distension presented during a negative emotional context was perceived more intensely, induced greater anxiety, and was associated with increased cortical activity, especially the right insula, compared with distension presented during a neutral emotional context. ATD also affects symptoms of anxiety since it has been shown that ATD significantly impairs the recognition of fearful facial expressions in females ${ }^{54}$, which suggests that subtle changes in the processing of emotional material may occur with this manipulation of serotonin function. This seems to be in accordance with our findings that ATD induced a significant shift in affective memory bias towards a preferential loss of positive material and enhanced visceral perception ${ }^{38}$. The present data suggest that ATD leads to a shift from primary visceral pain 
120 Chapter 6

sensation as represented by activation of the right insula and the inferior frontal operculum towards a more affective or emotional experience of the visceral pain sensation, as represented by increased activation of the anterior cingulate cortex, superior frontal cortex and right insula. The increased activation of the putamen may represent an increased availability for pain-related motor responses, such as flight behaviour.

Our data demonstrate that ATD leads to a small but significant shift in activation from the orbitofrontal cortex, the $A C C$ and insula towards more activation of the left insula during a non-painful rectal stimulus.

As the BOLD response is a function of blood flow, volume and blood oxygenation level, variability of these factors may affect the BOLD response. Since the reported ATD effects were restricted to specific area's associated with visceral perception, it is very unlikely that this increased cortical activation is a non-specific effect of ATD. This is further strengthened by the fact that there were no differences between ATD and placebo in cortical activation during the 30 seconds rest intervals of the stimulus paradigm.

In the present study we only included female volunteers. Consequently results might be representative for a female population only. The effects of ATD on $B O L D$ after visceral stimulation may be different in a male population ${ }^{21}$.

In summary, this study provides evidence that serotonergic modulation by ATD alters central processing of painful visceral stimuli towards a more affective experience of the visceral pain sensation. The results emphasize the important role of serotonin in brain-gut responses. 


\section{References}

1. Mayer EA. Emerging disease model for functional gastrointestinal disorders. Am J Med 1999; 107:12S-19S.

2. Mayer EA, Gebhart GF. Basic and clinical aspects of visceral hyperalgesia. Gastroenterology 1994;107:271-293.

3. Walker EA, Roy-Byrne PP, Katon WJ, Li L, Amos D, Jiranek G. Psychiatric illness and irritable bowel syndrome: a comparison with inflammatory bowel disease. Am J Psychiatry 1990;147:1656-1661.

4. Jackson JL, O'Malley PG, Tomkins G, Balden E, Santoro J, Kroenke K. Treatment of functional gastrointestinal disorders with antidepressant medications: a meta-analysis. Am J Med 2000;108:65-72.

5. Kim DY, Camilleri M. Serotonin: a mediator of the brain-gut connection. Am J Gastroenterol 2000;95:2698-2709.

6. Kilkens TO, Honig A, Rozendaal N, Van Nieuwenhoven MA, Brummer RJ. Serotonergic modulators in the treatment of irritable bowel syndrome - influence on psychiatric and gastrointestinal symptoms. Aliment Pharmacol Ther 2003;17:43-51.

7. Peyron $R$, Laurent $B$, Garcia-Larrea $L$. Functional imaging of brain responses to pain. $A$ review and meta-analysis (2000). Neurophysiol Clin 2000;30:263-288.

8. Baciu MV, Bonaz BL, Papillon E, Bost RA, Le Bas JF, Fournet J, Segebarth CM. Central processing of rectal pain: a functional MR imaging study. AJNR Am J Neuroradiol 1999;20: 1920-1924.

9. Berman S, Munakata J, Naliboff BD, Chang L, Mandelkern M, Silverman D, Kovalik E, Mayer EA. Gender differences in regional brain response to visceral pressure in IBS patients. Eur $J$ Pain 2000:4:157-172.

10. Berman SM, Chang $L$, Suyenobu B, Derbyshire SW, Stains J, Fitzgerald $L$, Mandelkern $M$, Hamm L, Vogt B, Naliboff BD, Mayer EA. Condition-specific deactivation of brain regions by 5-HT3 receptor antagonist Alosetron. Gastroenterology 2002;123:969-977.

11. Bernstein CN, Frankenstein UN, Rawsthorne P, Pitz M, Summers R, McIntyre MC. Cortical mapping of visceral pain in patients with $\mathrm{Gl}$ disorders using functional magnetic resonance imaging. Am J Gastroenterol 2002;97:319-327.

12. Chang L, Berman S, Mayer EA, Suyenobu B, Derbyshire S, Naliboff B, Vogt B, FitzGerald L, Mandelkern MA. Brain responses to visceral and somatic stimuli in patients with irritable bowel syndrome with and without fibromyalgia. Am J Gastroenterol 2003;98:1354-1361.

13. Derbyshire SW. A systematic review of neuroimaging data during visceral stimulation. Am $J$ Gastroenterol 2003;98:12-20.

14. Kern MK, Birn RM, Jaradeh S, Jesmanowicz A, Cox RW, Hyde JS, Shaker R. Identification and characterization of cerebral cortical response to esophageal mucosal acid exposure and distention. Gastroenterology 1998;115:1353-1362.

15. Kern MK, Shaker R. Cerebral cortical registration of subliminal visceral stimulation. Gastroenterology 2002;122:290-298.

16. Kern $M$, Hofmann $C$, Hyde J, Shaker R. Characterization of the cerebral cortical representation of heartburn in GERD patients. Am J Physiol Gastrointest Liver Physiol 2004; 286:G174-181.

17. Mayer EA, Derbyshire $S$, Naliboff BD. Cerebral activation in irritable bowel syndrome. Gastroenterology 2000;119:1418-1420.

18. Mayer EA, Berman S, Derbyshire SW, Suyenobu B, Chang L, Fitzgerald L, Mandelkern M, Hamm L, Vogt B, Naliboff BD. The effect of the 5-HT3 receptor antagonist, alosetron, on brain responses to visceral stimulation in irritable bowel syndrome patients. Aliment Pharmacol Ther 2002;16:1357-1366.

19. Mertz $H$, Morgan $V$, Tanner G, Pickens D, Price R, Shyr $Y$, Kessler R. Regional cerebral activation in irritable bowel syndrome and control subjects with painful and nonpainful rectal distention. Gastroenterology 2000;118:842-848. 
20. Naliboff BD, Derbyshire SW, Munakata J, Berman S, Mandelkern M, Chang L, Mayer EA. Cerebral activation in patients with irritable bowel syndrome and control subjects during rectosigmoid stimulation. Psychosom Med 2001;63:365-375.

21. Naliboff BD, Berman $S$, Chang $L$, Derbyshire SW, Suyenobu $B$, Vogt $B A$, Mandelkern $M$, Mayer EA. Sex-related differences in IBS patients: central processing of visceral stimuli. Gastroenterology 2003;124:1738-1747.

22. Silverman DH. Cerebral Activity in the Perception of Visceral Pain. Curr Rev Pain 1999;3: 291-299.

23. Silverman DH, Munakata JA, Ennes $H$, Mandelkern MA, Hoh $C K$, Mayer EA. Regional cerebral activity in normal and pathological perception of visceral pain. Gastroenterology 1997:112:64-72.

24. Yuan $Y Z$, Tao RJ, $X u B$, Sun J, Chen KM, Miao $F$, Zhang $Z W, X u J Y$. Functional brain imaging in irritable bowel syndrome with rectal balloon-distention by using fMRI. World $J$ Gastroenterol 2003;9:1356-1360.

25. Drossman DA, Ringel $Y$, Vogt BA, Leserman J, Lin W, Smith JK, Whitehead W. Alterations of brain activity associated with resolution of emotional distress and pain in a case of severe irritable bowel syndrome. Gastroenterology 2003:124:754-761.

26. Ringel $Y$, Drossman DA, Turkington TG, Bradshaw $B$, Hawk TC, Bangdiwala $S$, Coleman RE, Whitehead WE. Regional brain activation in response to rectal distension in patients with irritable bowel syndrome and the effect of a history of abuse. Dig Dis Sci 2003;48: 1774-1781.

27. Hobday DI, Aziz Q, Thacker N, Hollander I, Jackson A, Thompson DG. A study of the cortical processing of ano-rectal sensation using functional MRI. Brain 2001;124:361-368.

28. Lotze M, Wietek B, Birbaumer N, Ehrhardt J, Grodd W, Enck P. Cerebral activation during anal and rectal stimulation. Neuroimage 2001;14:1027-1034.

29. Strigo IA, Duncan $\mathrm{GH}$, Boivin M, Bushnell MC. Differentiation of visceral and cutaneous pain in the human brain. J Neurophysiol 2003;89:3294-3303.

30. Moore P, Landolt H-P, Seifritz E, Clark C, Batthi T, Kelsoe J, Rapaport M, Gillin JC. Clinical and Physiological Consequences of Rapid Tryptophan Depletion. Neuropsychopharmacology 2000:23:601-622.

31. Nishizawa S, Benkelfat C, Young SN, Leyton M, Mzengeza S, de Montigny C, Blier P, Diksic $M$. Differences between males and females in rates of serotonin synthesis in human brain. Proc Natl Acad Sci U S A 1997;94:5308-5313.

32. Carpenter LL, Anderson GM. Acute changes in CSF 5-HIAA following oral paroxetine challenge in healthy humans. Neuropsycho Pharmacology 2003;28:339-347.

33. Klaassen Tnio, Riedel WJ, Deutz NE, van Someren A, van Praag HM. Specificity of the tryptophan depletion method. Psychopharmacology (Berl) 1999;141:279-286.

34. Van der Does AJ. The effects of tryptophan depletion on mood and psychiatric symptoms. J Affect Disord 2001;64:107-119.

35. Bell $C$, Abrams $J$, Nutt D. Tryptophan depletion and its implications for psychiatry. $\mathrm{Br} J$ Psychiatry 2001:178:399-405

36. Young SN, Smith SE, Pihl RO, Ervin FR. Tryptophan depletion causes a rapid lowering of mood in normal males. Psychopharmacology 1985;87:173-177.

37. Van Nieuwenhoven MA, Valks SD, Sobczak S, Riedel WJ, Brummer RJ. Acute tryptophan depletion slows gastric emptying in females. $\mathrm{Br} \mathrm{J}$ Nutr 2004;91:351-355.

38. Kilkens TO, Honig A, van Nieuwenhoven MA, Riedel WJ, Brummer RJ. Acute tryptophan depletion affects brain-gut responses in irritable bowel syndrome patients and controls. Gut 2004;53:1794-1800.

39. Ledochowski M, Sperner-Unterweger B, Fuchs D. Lactose malabsorption is associated with early signs of mental depression in females: a preliminary report. Dig Dis Sci 1998;43: 2513-2517.

40. Sobczak S, Riedel WJ, Booij I, Aan Het Rot M, Deutz NE, Honig A. Cognition following acute tryptophan depletion: difference between first-degree relatives of bipolar disorder patients and matched healthy control volunteers. Psychol Med 2002;32:503-515. 
41. van Eijk HM, Rooyakkers DR, Deutz NE. Rapid routine determination of amino acids in plasma by high-performance liquid chromatography with a 2-3 microns Spherisorb ODS II column. J Chromatogr 1993;620:143-148.

42. Sloots CE, Felt-Bersma RJ, Cuesta MA, Meuwissen SG. Rectal visceral sensitivity in healthy volunteers: influences of gender, age and methods. Neurogastroenterol Motil 2000;12: $361-368$.

43. Talairach J, Tournoux P. Co-planar stereotaxic atlas of the human brain 3-dimensional porportional system: Anapproach to cerebral imaging. Stuttgart: New York; 1988.

44. Verne GN, Himes NC, Robinson ME, Gopinath KS, Briggs RW, Crosson B, Price DD. Central representation of visceral and cutaneous hypersensitivity in the irritable bowel syndrome. Pain 2003;103:99-110.

45. Schreckenberger $M$, Siessmeier $T$, Viertmann $A$, Landvogt $C$, Buchholz HG, Rolke $R$, Treede $R D$, Bartenstein $P$, Birklein $F$. The unpleasantness of tonic pain is encoded by the insular cortex. Neurology 2005;64:1175-1183.

46. Mayberg HS, Liotti M, Brannan SK, McGinnis S, Mahurin RK, Jerabek PA, Silva JA, Tekell JL, Martin CC, Lancaster JL, Fox PT. Reciprocal limbic-cortical function and negative mood: converging PET findings in depression and normal sadness. Am J Psychiatry 1999;156: 675-682.

47. Mayberg HS, Brannan SK, Tekell JL, Silva JA, Mahurin RK, McGinnis S, Jerabek PA. Regional metabolic effects of fluoxetine in major depression: serial changes and relationship to clinical response. Biol Psychiatry 2000;48:830-843.

48. Critchley HD, Mathias CJ, Josephs O, O'Doherty J, Zanini S, Dewar BK, Cipolotti L, Shallice $T$, Dolan RJ. Human cingulate cortex and autonomic control: converging neuroimaging and clinical evidence. Brain 2003;126:2139-2152.

49. Price JL. Prefrontal cortical networks related to visceral function and mood. Ann N Y Acad Sci 1999;877:383-396.

50. Bingel U, Glascher J, Weiller C, Buchel C. Somatotopic representation of nociceptive information in the putamen: an event-related fMRI study. Cereb Cortex 2004;14:1340-1345.

51. Stancak A, Polacek H, Vrana J, Rachmanova R, Hoechstetter K, Tintra J, Scherg M. EEG source analysis and $\mathrm{FMRI}$ reveal two electrical sources in the fronto-parietal operculum during subepidermal finger stimulation. Neuroimage 2005;25:8-20.

52. Ness TJ, Gebhart GF. Visceral pain: a review of experimental studies. Pain 1990;41: 167-234.

53. Phillips ML, Gregory LJ, Cullen $S$, Coen $S, N g$ V, Andrew C, Giampietro V, Bullmore $E$, Zelaya F, Amaro E, Thompson DG, Hobson AR, Williams SC, Brammer M, Aziz Q. The effect of negative emotional context on neural and behavioural responses to oesophageal stimulation. Brain 2003;126:669-684.

54. Harmer CJ, Rogers RD, Tunbridge E, Cowen PJ, Goodwin GM. Tryptophan depletion decreases the recognition of fear in female volunteers. Psychopharmacology (Berl) 2003; 167:411-417. 


\section{Chapter 7}

The effects of an acute serotonergic challenge on brain-gut responses in irritable bowel syndrome patients and controls

Kilkens TO, Honig A, Fekkes D, Brummer RJ

Aliment Pharmacol Ther 2005; 22: 865-874 


\section{Abstract}

\section{Background}

Serotonin, a key denominator of the brain-gut axis is involved in the regulation of gastrointestinal function as well as cognition, mood and hypothalamic-pituitary-adrenal axis mediated neuroendocrine responses.

\section{Aim}

To assess the effects of an acutely increased serotonergic activity, using a $20 \mathrm{mg}$ intravenous citalopram challenge test on visceral perception, affective memory performance, mood and neuroendocrine responses, respectively, in diarrhoea-predominant irritable bowel syndrome patients and controls.

\section{Methods}

In a randomised, double-blind crossover design, fourteen diarrhoea-predominant irritable bowel syndrome patients and fourteen matched controls were studied under citalopram and placebo conditions, respectively. Visceral perception was scored in response to rectal distensions. Affective memory performance, mood, levels of adrenocorticotropic hormone, cortisol, prolactin and biochemical parameters of serotonergic metabolism were simultaneously assessed.

\section{Results}

Visceral perception did not significantly differ between the citalopram and placebo condition. Citalopram administration enhanced affective memory performance because of a bias towards positive material but no significant changes in mood. Citalopram significantly increased plasma serotonin, adrenocorticotropic hormone and cortisol levels compared with placebo. Citalopram did not differentially affect the patient or control group.

\section{Conclusions}

We have provided evidence that acutely increased serotonergic activity influences neuroendocrine responses and cognition in diarrhoea-predominant irritable bowel syndrome and controls without a significant effect on visceral perception. 


\section{Introduction}

The pathophysiology of irritable bowel syndrome (IBS) is not fully understood. A multi-component conceptual model of IBS has been postulated, involving physiological, affective, cognitive and behavioural factors ${ }^{1}$.The 'brain-gut axis' plays a major role in the concept of IBS. It is a theoretical model describing the bidirectional neural pathways linking cognitive and emotional centres in the brain to neuroendocrine centres, the enteric nervous system and the immune system $^{2}$. IBS is associated with visceral hypersensitivity and with a high cooccurrence of psychiatric symptoms, in particular affective dysregulation ${ }^{3,4}$.

Serotonin (5-hydroxytryptamine, 5-HT) is a biogenic amine that functions among others as a neurotransmitter and is located predominantly in the gastrointestinal $(\mathrm{Gl})$ tract $(80 \%$ of total body $5-\mathrm{HT})$. Approximately $5 \%$ is located in the brain and cerebrospinal fluid (CSF). Peripherally, 5-HT is involved in the regulation of $\mathrm{Gl}$ secretion, motility and perception, whereas in the central nervous system (CNS) it plays a role in the regulation of mood and cognition ${ }^{5,6}$. Hence, $5-\mathrm{HT}$ is a key denominator of the 'brain-gut axis'

Disturbed serotonergic metabolism seems especially prevalent in the diarrhoea-predominant type of IBS (d-IBS) ${ }^{7,8}$. Recently, we demonstrated that acute lowering of 5-HT synthesis was associated with altered brain-gut responses, i.e. enhanced visceral perception and impaired affective memory performance in d-IBS patients and healthy controls ${ }^{9}$.

Stimulation of serotonergic neurotransmission induces an hypothalamicpituitary-adrenal (HPA) axis-mediated neuroendocrine response ${ }^{10}$. An acute increase of 5-HT activity can be achieved by intravenous (i.v.) administration of the selective serotonin reuptake inhibitor (SSRI) citalopram ${ }^{11}$. The neuroendocrine response induced by citalopram challenge has been used in a psychiatric setting as an indirect measure of central serotonergic function ${ }^{10}$. In patients with affective disorders alterations in the neuroendocrine response to serotonergic stimulation have been shown ${ }^{12}$. It is unknown whether such alterations in central serotonergic function are present in d-IBS patients ${ }^{13,14}$.

The above findings indicate that citalopram challenge offers a potential technique to study the contribution of 5-HT to the regulation of the 'brain-gut axis'. This study describes the effects of acute serotonergic modulation, using the citalopram challenge test, at the level of both the brain and the gut, in patients with d-IBS and healthy controls. We applied this model to investigate whether: (i) citalopram challenge influences visceral perception during rectal distensions and (ii) whether citalopram challenge simultaneously influences 
$123 \mid$ Onapter 7

affective memory performance, mood and neuroendocrine responses. Based on our previous findings that acute lowering of 5-HT was associated with enhanced visceral perception and impaired affective memory performance, we hypothesized that an increased serotonergic activity would be associated with decreased visceral perception and improved affective memory performance.

\section{Materials and methods}

\section{Subjects}

Subjects were screened comprising a standardized psychiatric examination using the Mini International Neuropsychiatric Interview (MINI) ${ }^{15}$ to determine the present psychiatric state. General psychological state was assessed using the 17-item Hamilton Depression Rating Scale (HAM-D17) ${ }^{16}$, the Dutch version of the symptom checklist (SCL-90) ${ }^{17}$ and the Hospital Anxiety and Depression Scale $(\mathrm{HADS})^{18}$. Physical health was assessed by means of a standard physical examination and a urine test. A pregnancy test was performed in all female subjects. Intelligence was estimated using the Groninger Intelligence Test $(\mathrm{GIT})^{19}$.

The Medical Ethics Committee of the University Hospital Maastricht approved the study protocol and all subjects gave their written informed consent before start of the study. Exclusion criteria were abdominal surgery (other than appendectomy and cholecystectomy), use of medications other than oral contraceptives within fourteen days prior to testing, lactose malabsorption ${ }^{20}$, a positive first-degree psychiatric family history ${ }^{21}$, any history of psychiatric disease or use of psychoactive medication, premenstrual syndrome, dieting, pregnancy or lactation, excessive alcohol intake $(>20$ alcoholic consumptions per week) and hypertension (diastolic $>100 \mathrm{mmHg}$, systolic $>170 \mathrm{mmHg}$ ), respectively. Additional exclusion criteria for the control subjects were current or history of GI disorder, current psychiatric or psychological symptomatology defined as a diagnosis on the MINI, HAM-D17 scores above 18, global severity index (GSI) score on SCL-90 for females $\geq 150$ and for males $\geq 131$ or HADS scores $\geq 8$, respectively. The required number of subjects to be included was based on pilot experiments and an estimated effect size of 1.0 (calculated from the standardized mean difference between the experimental and placebo condition with respect to the primary outcome parameter), with $\beta=0.2$ and $\alpha=0.05$. Fourteen patients fulfilled the diagnosis of $d-I B S$ according to the ROME II criteria, diagnosed by an experienced gastroenterologist ${ }^{22}$ and fourteen healthy matched control subjects were included (Table 7.1). Prior to the test days a training version of the affective memory performance test was practiced to minimize learning effects. 
Table 7.1 Subject characteristics (median (range)).

\begin{tabular}{lccc}
\hline & $\begin{array}{c}\text { d-IBS patients } \\
n=14\end{array}$ & $\begin{array}{c}\text { Controls } \\
n=14\end{array}$ & $\begin{array}{c}\text { Patients vs. Controls } \\
\text { p value }\end{array}$ \\
\hline Females $(n)$ & $8 / 14$ & $8 / 14$ & - \\
Oral Contraceptives $(n)$ & $6 / 8$ & $6 / 8$ & - \\
Age & $31.5(18-52)$ & $32.5(19-57)$ & 0.839 \\
Intelligence quotient & $112(88-122)$ & $116(95-135)$ & 0.352 \\
Body mass index & $21.9(18-35)$ & $22.8(19-26)$ & 0.701 \\
Alcohol units/day & $0.22(0-2)$ & $0.35(0-1)$ & 0.329 \\
Cigarettes/day & $0.2(0-5)$ & $0.0(0-10)$ & 0.352 \\
Diagnosis on MINI & $5^{\mathrm{b}}$ & 0 & - \\
HAM-D17 & $3.5(0-11)$ & $1.0(0-4)$ & $0.007^{\mathrm{a}}$ \\
SCL-90 (GSI) & $111(91-170)$ & $93.5(90-128)$ & $0.004^{\mathrm{a}}$ \\
HADS (total) & $7.5(1-10)$ & $2.0(0-8)$ & $0.004^{\mathrm{a}}$ \\
\hline
\end{tabular}

d-IBS, diarrhoea-predominant IBS; MINI, Mini International Neuropsychiatric Interview; HAM-D17, 17-item Hamilton Depression Rating Scale; SCL-90, Symptom Check List; GSI, Global Severity Index; HADS, Hospital Anxiety and Depression Scale. ${ }^{a}$ Indicating a significant difference between groups. ${ }^{b}$ Five subjects fulfilled the criteria of a psychiatric diagnosis: two panic disorder and agoraphobia; two agoraphobia; one depression, social phobia and anxiety disorder, respectively. ${ }^{c}$ Mann-Whitney test comparing $d$-IBS patients versus control subjects.

\section{Study design}

The study was conducted following a randomised placebo-controlled, doubleblind, crossover design (Figure 7.1). The two test days were spaced a minimum of fourteen days apart. All women were tested in the follicular phase of the menstrual cycle or while taking oral contraception ${ }^{23}$. All subjects were tested within 3 months time to avoid possible seasonal variation. They were asked to abstain from heavy physical exercise and consumption of alcoholic beverages the day prior to their visit. All subjects attended the laboratory twice after an overnight fast (after 10:00 PM no eating, drinking or smoking allowed) at 8:00 AM. At 8:15 AM an i.v. catheter was placed in a forearm vein. Between $9.00 \quad(t=0)$ and 9.15 AM subjects received an i.v. infusion (citalopram or placebo). Assessment of visceral perception was started at $t=40$ minutes and affective memory performance at $t=90$ minutes, respectively. Mood was assessed each test day at $t=-60,0,55,75$ and 135 minutes, respectively. Blood samples were taken at $t=0,15,30,45,60,75$ and 90 minutes, respectively. 
$130 \mid$ Chapter 7

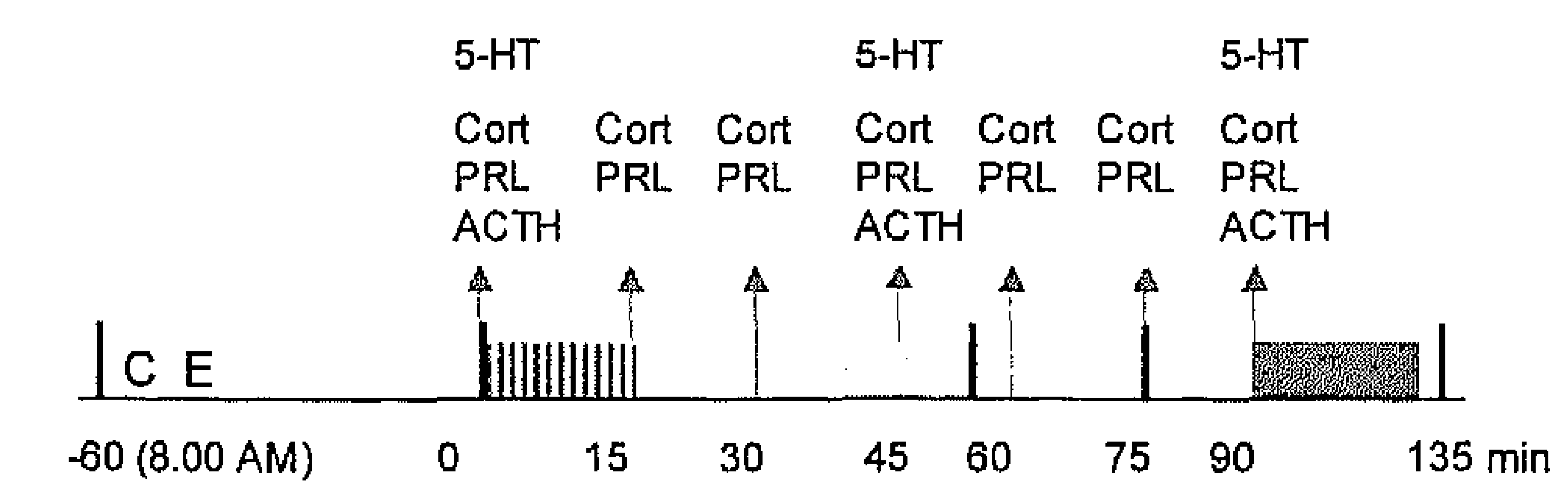

1 mood assessment

$E$ enema

A blood sampling

竘 affective memory performance

IIIII i.v. infusion

visceral perception

C insertion i.v. catheter

Figure 7.1 Schematic representation of a test day (flow chart).

\section{Citalopram challenge}

The i.v. catheter was kept open by $0.9 \%$ saline solution. Subjects received an i.v. infusion over a period of $15 \mathrm{~min}$ containing citalopram $(20 \mathrm{mg}$ citalopram diluted in $100 \mathrm{ml}$ of $0.9 \%$ saline solution) or placebo $(100 \mathrm{ml}$ of $0.9 \%$ saline solution) using a calibrated volumetric infusion pump ${ }^{12}$.

\section{Visceral perception}

The bowel preparation consisted of a tap water enema 1 hour before start of the citalopram administration. A detailed description regarding the barostat device and the standardized barostat protocol has been described elsewhere ${ }^{9}$. In short, the protocol consisted of intermittent semi-random staircase distensions of 60 seconds duration $(15,10,25,20 \mathrm{mmHg}$ etc.) separated by an interval of 30 seconds baseline pressure. During each distension subjects were asked to report their perception of urge and pain, respectively. Urge was scored: 1 , not perceptible; 2 , first perception; 3 , minor urge to defecate; 4 , normal urge to defecate; 5 , strong urge to defecate and 6 , maximal tolerable urge. Pain was scored using a $100 \mathrm{~mm}$ visual analogue scale (no pain-maximal tolerable pain). Rectal volume was measured at the end of each distension. Apparent rectal compliance $(\Delta \mathrm{V} / \Delta \mathrm{P} ; \mathrm{P}=10-45 \mathrm{mmHg})$ was estimated for each subject.

\section{Cognition and mood}

The affective memory test consisted of a list of 30 emotionally loaded stimulus words (twelve positive, twelve negative and six neutral) ${ }^{24}$. All 30 words of the 
list were consecutively presented three times on a computer screen. Each presentation of the list ended with a free recall of the words. Recall scores were summed to comprise the total immediate recall score. After the third presentation, subjects performed a 30-minute distraction task and subsequently the subject was requested to recall as many of the previously learned words as possible (delayed recall). Outcome measures were the percentages of positive, negative and neutral words recalled of the total immediate recall and delayed recall score, respectively. Mood was assessed with visual analogue scales adapted from the profile of mood states (POMS) ${ }^{25}$. It consisted of 32 items describing bipolar mood adjectives (e.g. happy - sad). Items were grouped to form measures of five mood dimensions: depression, tension, vigour, anger and fatigue, respectively.

\section{Biochemical parameters}

Concentrations of 5-HT were determined in platelet poor plasma (ppp) and were regarded as markers of peripheral 5-HT metabolism? ${ }^{7}$. Blood for adrenocorticotropic hormone (ACTH) and 5-HT measurements was sampled in Vacutainer EDTA tubes (Becton Dickinson, Meylan, France). Blood for 5-HT determinations was kept at room temperature and centrifuged within one hour $\left(20\right.$ minutes, $2600 \mathrm{~g}, 20^{\circ} \mathrm{C}$ ). As a control of the ppp preparation procedure, platelets were counted in the ppp using a Coulter Counter (Coulter MD Series, Coulter Corporation, Hialeah, Florida, USA). Blood for serum cortisol and prolactin measurement was sampled in Vacutainer SST tubes. Blood samples for $\mathrm{ACTH}$, cortisol and prolactin were kept at $0^{\circ} \mathrm{C}$ and were immediately centrifuged (15 minutes, $2600 \mathrm{~g}, 4^{\circ} \mathrm{C}$ ). Ppp 5- $\mathrm{HT}^{26,27}, \mathrm{ACTH}$, cortisol and prolactin samples were analysed as previously described ${ }^{28}$.

\section{Statistical analysis}

Primary outcome parameter was visceral perception and secondary outcome parameters were rectal compliance, affective memory performance, mood and neuroendocrine parameters. Data are presented as mean (SE) except for subject characteristics [median (range)] because of the absence of normal distribution. Differences in subject characteristics were analysed using Wilcoxon Mann-Whitney test. Outcome variables were analysed using ANOVA for repeated measures. For affective memory performance the within subjects factor was treatment (two levels: citalopram, placebo) whereas for the biochemical parameters the within-subjects factors were treatment (two levels: citalopram, placebo) and time (for $5-\mathrm{HT}$ and ACTH three levels: $\mathrm{t}=0,45$ and 90 minutes and for cortisol and prolactin seven levels: $t=0,15,30,45,60,75$ and 90 minutes, respectively). Urge and pain perception were analysed in the $10-45 \mathrm{mmHg}$ range, as our total number of subjects became too small for 
adequate statistical analysis at pressures above $45 \mathrm{mmHg}$. For the visceral perception and mood analyses within-subjects factors were treatment (two levels: citalopram, placebo), pressure (eight levels: 10, 15, 20, 25, 30, 35, 40 and $45 \mathrm{mmHg}$ ) and time (five levels: $\mathrm{t}=-60,0,55,75$ and 135 minutes), respectively. The between-subjects factor for all outcome variables was diagnosis of d-IBS (two levels: d-IBS, controls). A potential differential treatment response between subject groups was assessed by analysing the treatment $x$ diagnosis of $d$-IBS interaction. In case of a slight violation of sphericity, the Greenhouse-Geisser correction was applied. A p-value $<0.05$ was considered statistically significant. Statistical analyses were performed using the SPSS 10.0 for Windows software package.

\section{Results}

\section{Visceral perception}

Urge and pain (Figures 7.2, 7.3). Urge and pain scores increased significantly with increasing pressure $[F(7,147)=172, p<0.001 ; F(7,147)=4.6, p<0.001$, respectively]. Pain scores were significantly increased in the patient group compared with the controls $[F(1,21)=6.49, p<0.05]$, which confirms the presence of visceral hypersensitivity in our d-IBS population.

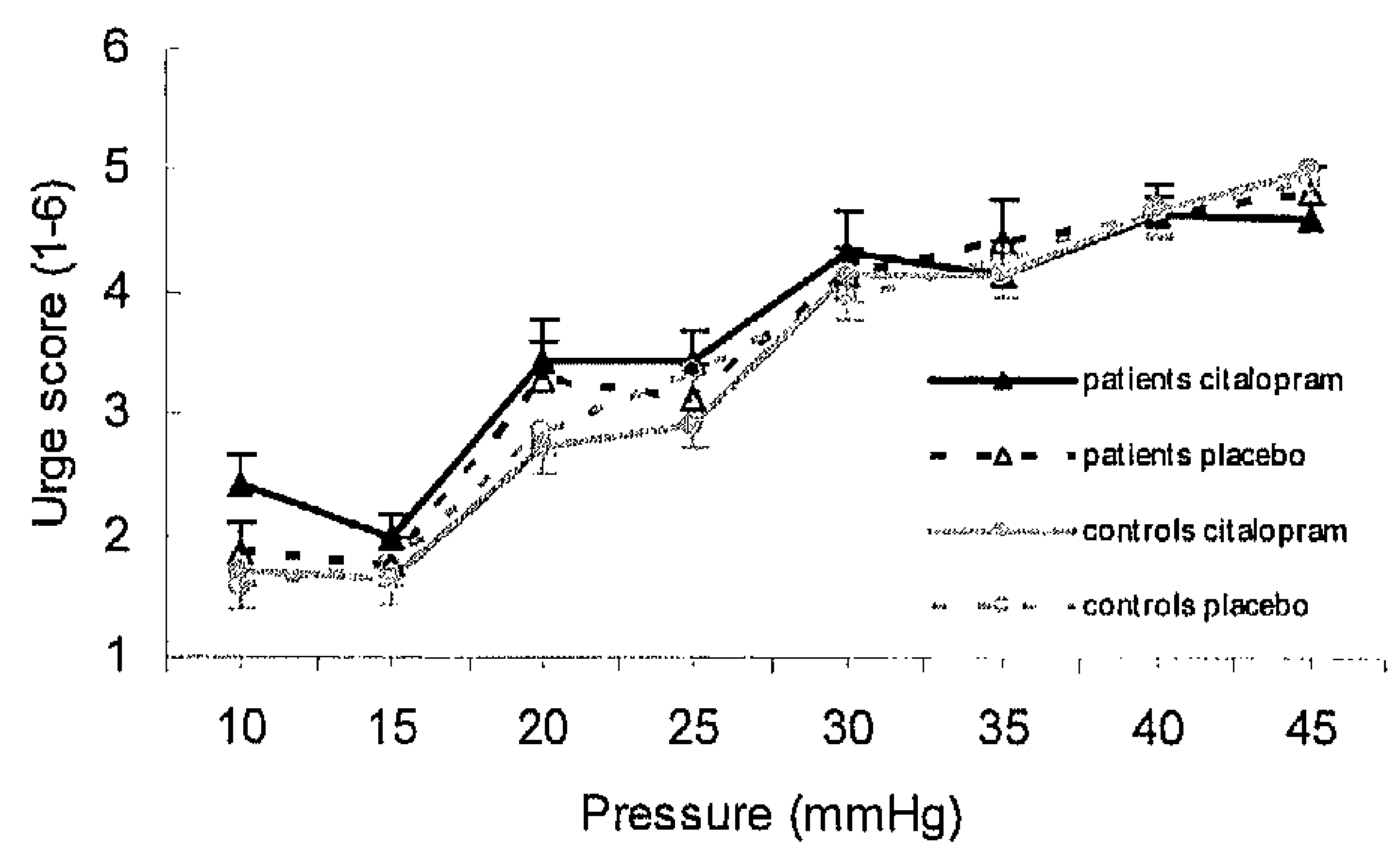

Figure 7.2 Pressure-urge scores (mean (SE)) during intermittent pressure distension of the rectum during intravenous (i.v.) citalopram challenge and placebo, in diarrhoeapredominant irritable bowel syndrome (d-IBS) patients and control subjects respectively. Citalopram did not significantly affect urge scores compared with placebo $[F(1,21)=0.93, p=0.3]$. 


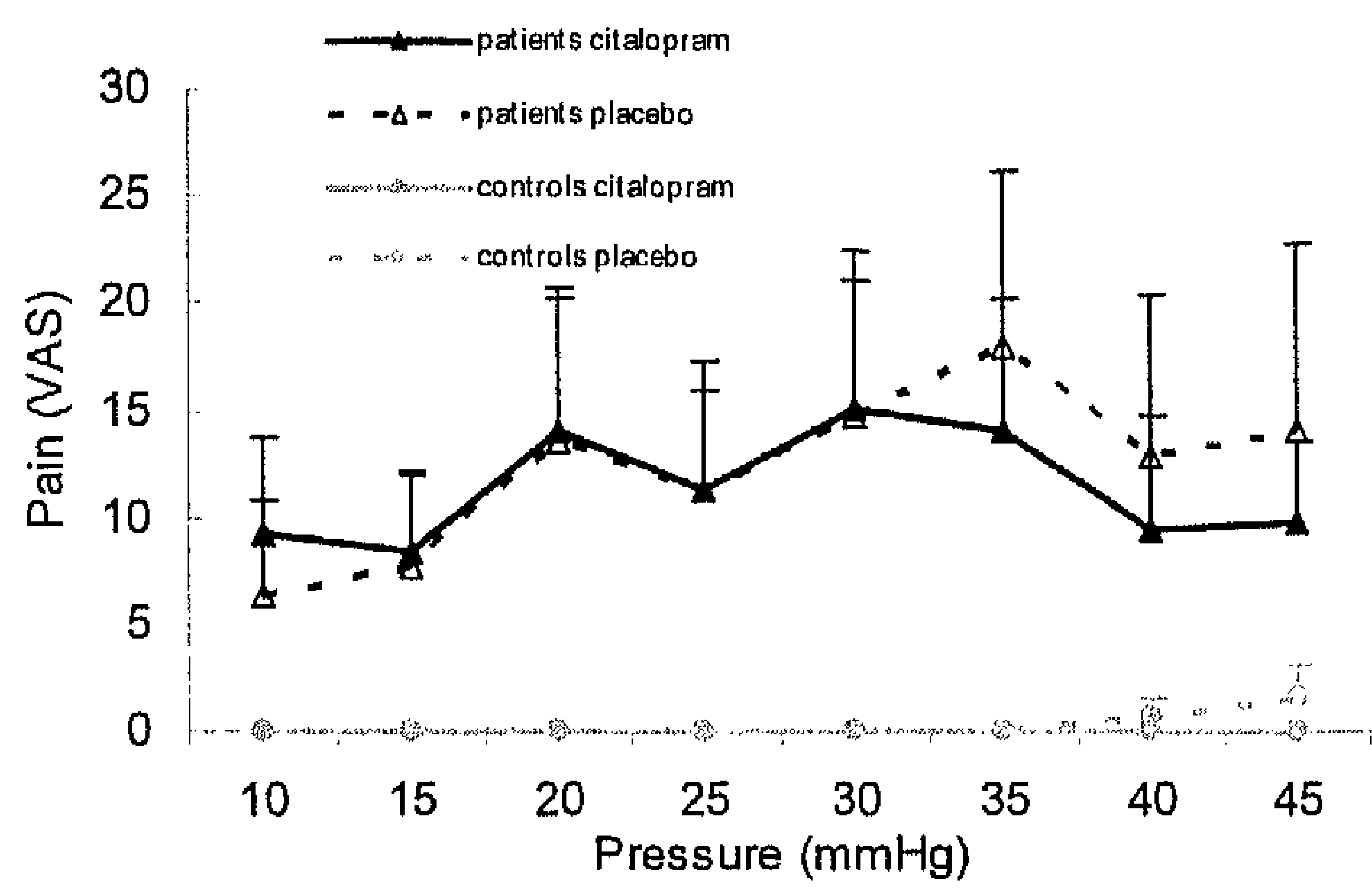

Figure 7.3 Pressure-pain scores (mean (SE)) during intermittent pressure distension of the rectum during intravenous (i.v.) citalopram challenge and placebo, in diarrhoeapredominant irritable bowel syndrome (d-IBS) patients and control subjects respectively. Citalopram did not significantly affect pain scores compared with placebo $[F(1,21)=2.1, p=0.2]$.

Citalopram administration was not significantly associated with differences in overall urge or pain scores compared with placebo $[F(1,21)=0.93, p=0.3$ and $F(1,21)=2.1, p=0.2$, respectively]. The effects of citalopram on urge and pain scores did not significantly differ between the patients and controls.

Rectal compliance. Patients showed significantly decreased rectal volumes compared with controls $[F(1,24)=5.53, p<0.05]$. There was no significant difference in pressure-volume relation or rectal compliance during citalopram compared with placebo $[F(1,24)=0.03, p=0.9 ; \quad F(1,26)=0.00, p=1.0$, respectively].

\section{Cognition and mood}

Patients had decreased total immediate memory performance scores compared with controls $(p<0.05)$ (Table 7.2). Overall, citalopram administration was associated with improved total immediate recall performance $[F(1,26)=3.13, p<0.05]$ compared with placebo. Citalopram did not differentially affect the patient and the control group at a significant level [no treatment $x$ diagnosis of $d$-IBS interaction, $F(1,26)=2.23, p=0.15$ ]. Post hoc analyses indicated that the increased total recall score was due to improved recall of positive words $[F(1,26)=4.99, p<0.05]$. Immediate recall of negative and neutral words was not significantly affected by citalopram.

Patients had decreased total delayed memory performance scores compared with controls $(p<0.05)$. Citalopram tended to be associated with improved total 
$134 \mid$ Chapter 7

delayed recall performance $[F(1,26)=2.34, p=0.07]$ compared with placebo, although this difference did not reach significance. Citalopram did not differentially affect the patient and the control group. Post hoc analyses indicated that this trend towards increased total recall scores was due to a tendency towards improved recall of positive words $[F(1,26)=2.30, p=0.09]$. Immediate recall of negative and neutral words was not significantly affected by citalopram.

There were no significant differences in mood between subject groups or treatment conditions $(p>0.2)$.

Table 7.2 Influence of i.v. citalopram administration compared with placebo on affective memory performance, in d-IBS patients and control subjects, respectively. Mean (SE) of percentages of positive, negative and neutral words recalled immediately (total immediate recall) and recalled after a delay of 30 minutes (delayed recall).

\begin{tabular}{llllc}
\hline & & d-IBS patients & Controls & $\begin{array}{c}\text { Citalopram vs. Placebo } \\
\text { p value }\end{array}$ \\
\hline Immediate recall & Citalopram & $35.9(4.3)$ & $51.3(5.0)$ & $0.044^{*}$ \\
Total & Placebo & $35.3(3.2)$ & $44.8(4.6)$ & \\
Positive words & Citalopram & $37.9(5.2)$ & $52.8(4.4)$ & $0.017^{*}$ \\
& Placebo & $36.5(3.9)$ & $40.5(4.4)$ & \\
Negative words & Citalopram & $32.5(4.5)$ & $49.2(6.0)$ & 0.67 \\
& Placebo & $32.9(3.8)$ & $46.6(5.1)$ & \\
Neutral words & Citalopram & $38.5(4.6)$ & $52.8(7.0)$ & 0.54 \\
& Placebo & $37.7(3.9)$ & $49.6(5.6)$ & \\
Delayed recall & & & & \\
Total & Citalopram & $36.2(6.4)$ & $57.1(6.5)$ & 0.069 \\
& Placebo & $33.6(4.0)$ & $50.2(7.0)$ & \\
Positive words & Citalopram & $38.1(6.9)$ & $59.5(6.0)$ & 0.089 \\
& Placebo & $35.1(5.9)$ & $49.4(7.1)$ & \multirow{2}{*}{0.185} \\
Negative words & Citalopram & $33.3(6.7)$ & $54.8(7.1)$ & \multirow{2}{*}{0.774} \\
& Placebo & $40.0(4.4)$ & $46.4(7.8)$ & \\
Neutral words & Citalopram & $38.1(8.1)$ & $57.1(8.0)$ & \\
& Placebo & $35.7(5.8)$ & $59.5(8.7)$ & \\
\hline
\end{tabular}

*Indicating a significant treatment effect; i.v., intravenous; d-IBS, diarrhoea-predominant irritable bowel syndrome.

\section{Biochemical parameters}

Absolute ppp 5-HT levels did not significantly differ between the subject groups at baseline (patients $10.5(1.9) ; 9.65(1.7)$, controls $13.2(3.2) ; 7.1(1.4) \mathrm{nmol} / /$, in the citalopram and placebo condition respectively). The $\mathrm{ppp} 5-\mathrm{HT}$ concentrations increased significantly in the citalopram condition compared with placebo $[F(2,52)=29.1, p<0.001]$. This effect did not significantly differ between patients and controls $(p=0.2)$ (Figure 7.4). Absolute ACTH levels did 
not significantly differ between groups at baseline (patients $22.6(2.6) ; 27.6$ (3.3), controls $23.0(2.3) ; 23.1(2.3) \mathrm{ng} / \mathrm{l}$ in the citalopram and placebo condition respectively). The ACTH concentrations increased significantly in the citalopram condition compared with placebo $[F(2,52)=16.5, p<0.001]$. This effect did not significantly differ between the two subject groups $(p>0.6$; Figure 7.5). The d-IBS patients had significantly increased absolute cortisol levels compared with controls at baseline $[F(1,26)=4.73, p<0.05]$ (patients $584(55)$; 599 (52), controls $454(31) ; 491$ (44) $\mathrm{nmol} / /$ in the citalopram and placebo condition respectively). The cortisol concentrations increased significantly in the citalopram condition compared with placebo $[F(6,150)=5.04, p<0.001]$. This effect did not significantly differ between patients and controls $(p=0.6$; Figure 7.6). Absolute prolactin levels did not significantly differ between groups at baseline. The prolactin concentrations did not significantly increase in the citalopram condition compared with placebo $[F(6,144)=1.36, p=0.2]$.

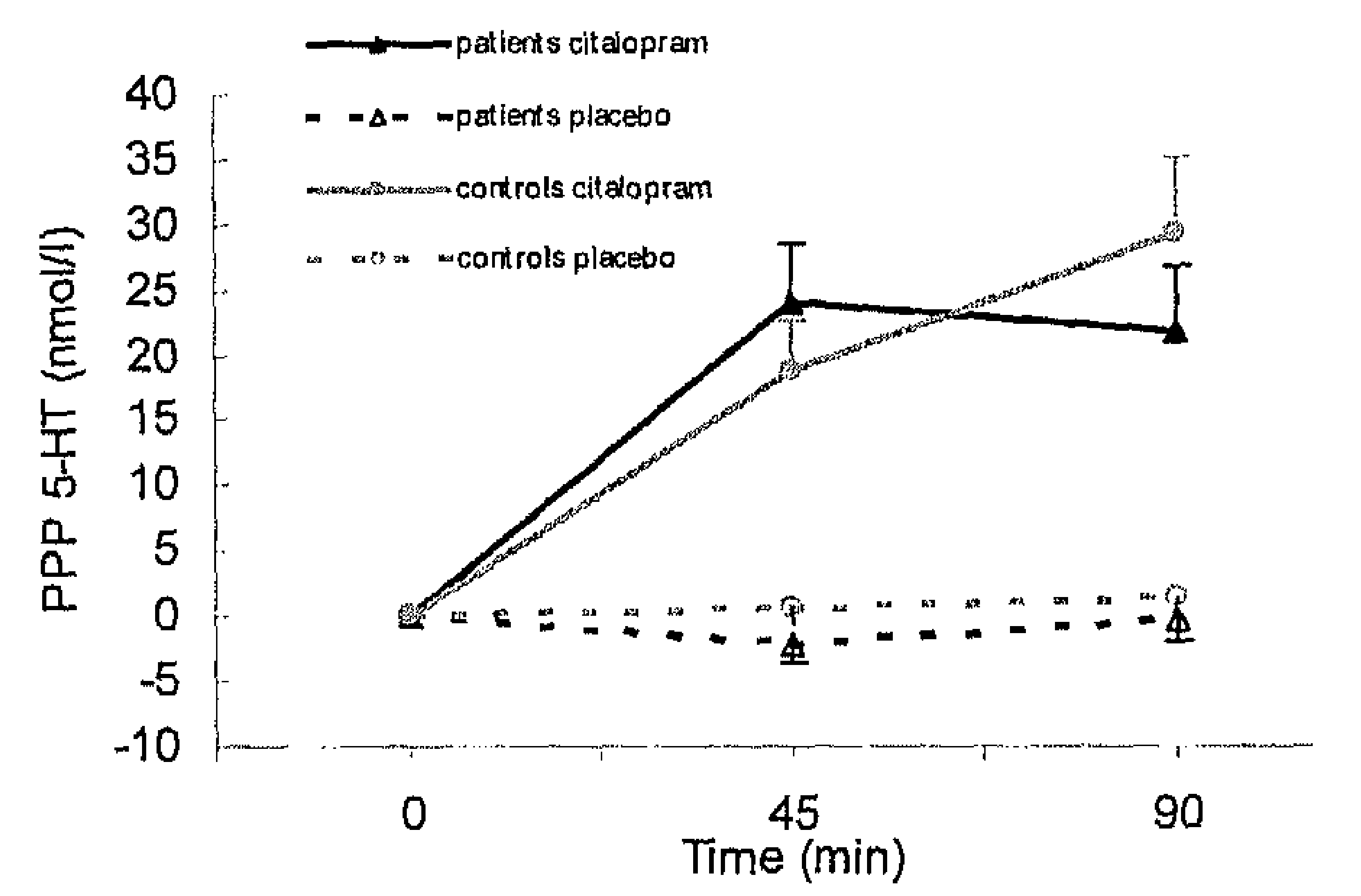

Figure 7.4 Mean (SE) platelet poor plasma (ppp) 5-HT (measured as change from baseline) responses as a function of time in diarrhoea-predominant irritable bowel syndrome (dIBS) patients and control subjects respectively, receiving citalopram $20 \mathrm{mg}$ and placebo, respectively, infused intravenously over 15 minutes starting at time " 0 ". Citalopram significantly increased $5-H T$ levels compared with placebo $[F(2,52)=29.1$, $p<0.001]$.

\section{Post-hoc correlations}

In post-hoc analyses, correlations between level of affective dysregulation and citalopram induced ACTH and cortisol responses were investigated. Significant negative correlations between HAM-D17, SCL-90 and HADS scores with ACTH responses were found (Pearson's $r=-0.36, p<0.05 ; r=-0.53, p<0.01$ and $r=-0.60, p<0.001$ respectively). Significant negative correlations between SCL90 and HADS scores with cortisol responses were found (Pearson's $r=-0.33$, $p<0.05$ and $r=-0.31, p=0.05$ ). 


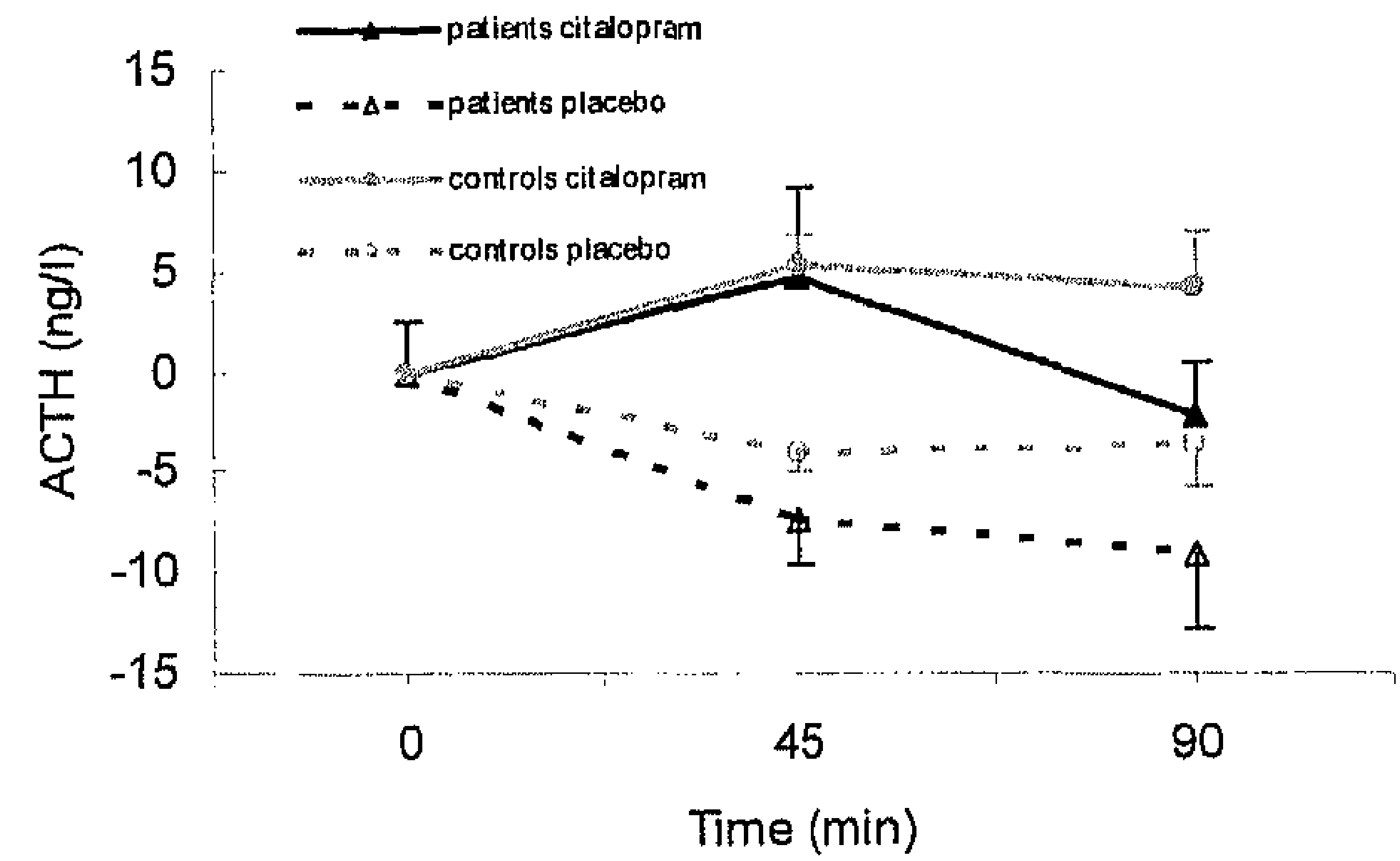

Figure 7.5 Mean (SE) plasma adrenocorticotropic hormone (ACTH; measured as change from baseline) responses as a function of time in diarrhoea-predominant irritable bowel syndrome (d-IBS) patients and control subjects respectively, receiving citalopram $20 \mathrm{mg}$ and placebo, respectively, infused intravenously over 15 minutes starting at time " 0 ". Citalopram significantly increased ACTH levels compared with placebo $[F(2,52)=16.5, p<0.001]$.

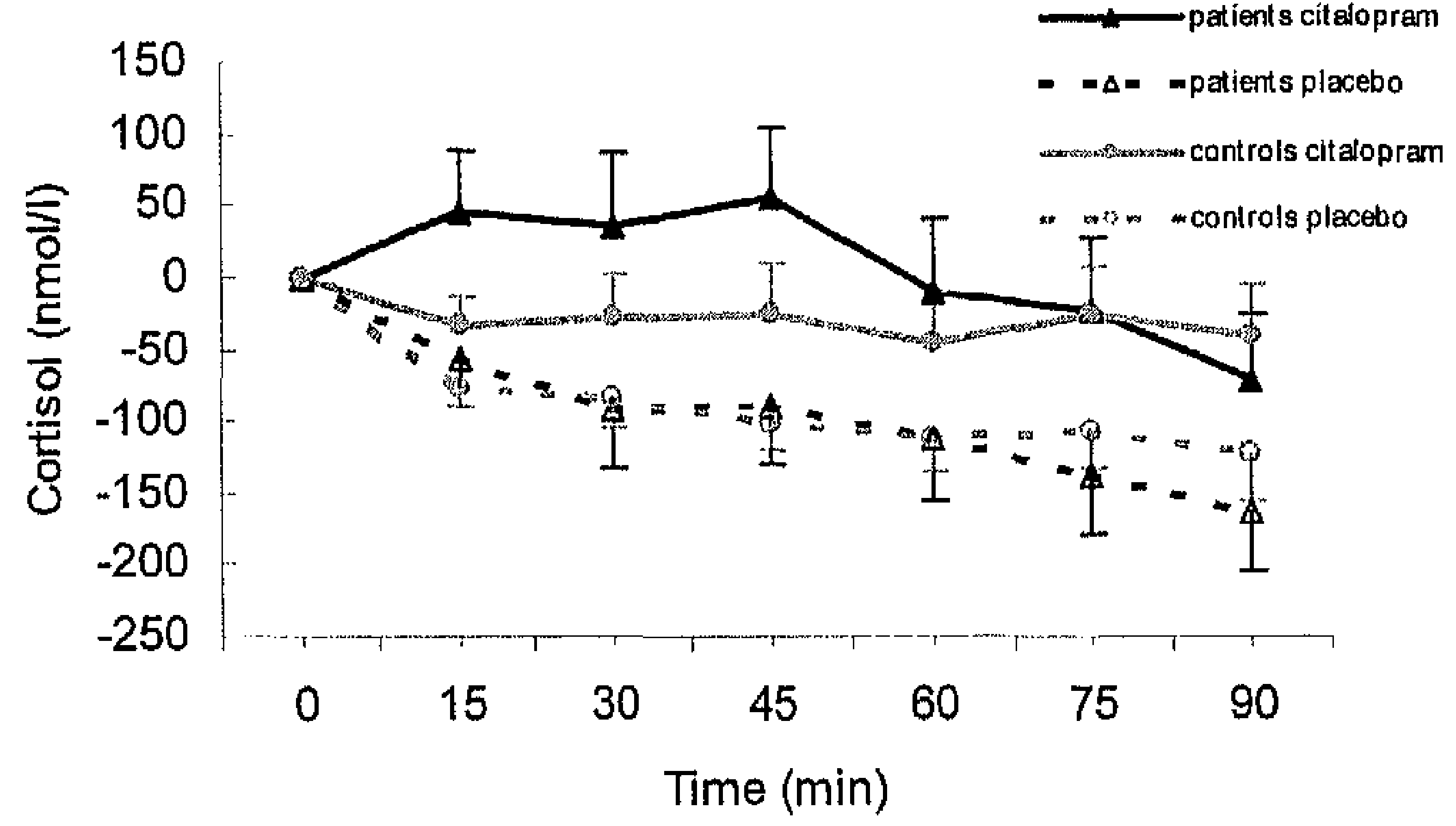

Figure 7.6 Mean (SE) serum cortisol (measured as change from baseline) responses as a function of time in diarrhoea-predominant irritable bowel syndrome (d-IBS) patients and control subjects respectively, receiving citalopram $20 \mathrm{mg}$ and placebo, respectively, infused intravenously over 15 minutes starting at time "0". Citalopram significantly increased cortisol levels compared with placebo $[F(6,150)=5.04, p<0.001]$.

\section{Discussion}

We have demonstrated that acutely increasing the serotonergic activity by selective serotonin reuptake inhibition does not significantly influence visceral urge or pain perception. However, the citalopram challenge was significantly associated with increased ACTH and cortisol levels and enhanced affective 
memory performance because of a bias towards positive material in d-IBS patients and healthy matched controls.

Citalopram, a widely used antidepressant, is the SSRI with the highest selectivity for the serotonin reuptake transporter (SERT), with no known intrinsic activity at any $5-\mathrm{HT}$ receptor subtype or at any other neurotransmitter system ${ }^{11}$.

Data concerning the role of SSRIs on visceral perception are conflicting. Reduced oesophageal sensitivity to distension has been described, but these drugs seem to lack this effect at gastric level ${ }^{29}$. This is the first study investigating the acute effects of an SSRI on brain and gut responses simultaneously. Two preliminary studies indicated that acute i.v. administration of $20 \mathrm{mg}$ citalopram did not alter the sensitivity to descending colonic pressure distension ${ }^{30,31}$. A single oral dose of another SSRI fluoxetine, did not modify rectal perception in healthy controls ${ }^{32}$. In addition, 6-week treatment with fluoxetine $20 \mathrm{mg}$ dd in 40 non-depressed IBS patients did not significantly change rectal sensitivity or compliance ${ }^{33}$. The role of specific 5 -HT modulators, mainly $5-\mathrm{HT}_{3}$ antagonists and $5-\mathrm{HT}_{4}$ agonists in visceral perception has been subject of earlier studies ${ }^{6}$. However, it is not feasible to compare our results with studies using receptor-specific 5-HT modulators, because both at the CNS as well as the Gl level, $5-\mathrm{HT}$ interacts with a number of different $5-\mathrm{HT}$ receptor subclasses that are either stimulatory or inhibitory. Although our barostat protocol was primarily designed to detect changes in visceral perception our data of altered rectal pressure-volume relations in d-IBS patients are in accordance with former studies ${ }^{9,34}$.

Citalopram significantly enhanced memory performance. These results are in line with a study of Harmer et al., which demonstrated that $10 \mathrm{mg}$ i.v. citalopram administration increased memory consolidation ${ }^{35}$. In addition to enhanced memory performance, citalopram induced an affective memory bias toward positive material without significantly influencing the subjective mood status, using the POMS. These results are in line with data from Harmer et al., which demonstrated that citalopram administration reduces the processing of negative relative to positive emotional material without notable mood differences ${ }^{36,37}$. Also, acute lowering of $5-\mathrm{HT}$ synthesis impaired memory performance with an affective memory bias towards a preferential loss of positive material without significant mood differences ${ }^{9}$. We stated that affective memory bias may offer a more objective and sensitive biomarker of central serotonergic regulation ${ }^{9}$. This seems to be confirmed by our current findings as well as by the findings of Kemp et al., which demonstrated that acute citalopram administration enhances pleasant, and suppresses unpleasant cortical electrophysiological responses to emotional images without altering 
subject's subjective responses ${ }^{38}$. In contrast to our previous study memory performance was significantly impaired in d-IBS compared with controls, ${ }^{9}$ however, in the present study memory performance was assessed after the barostat procedure. Possibly, the barostat procedure induced a different stress response in our IBS subjects compared with the controls which might have differentially affected memory performance ${ }^{39}$.

Consistent with other studies citalopram significantly increased ACTH and cortisol levels ${ }^{12,40,41}$. However, we did not demonstrate a significant effect of citalopram on plasma prolactin levels. Failure of oral citalopram administration to increase prolactin levels has been reported ${ }^{42,43}$. It was suggested that prolactin responses after i.v. drug administration may be a result of other than 5 -HT-mediated processes ${ }^{42}$. In addition, the effects of a barostat procedure on neuroendocrine responses remain unknown.

Increased urine and salivary cortisol levels have been reported in IBS ${ }^{44,45}$. In patients with affective disorders basal hypercortisolaemia and attenuated neuroendocrine responses after a serotonergic challenge have been reported ${ }^{12,46}$. We studied a homogenous group of d-IBS patients which demonstrated increased levels of affective dysregulation and increased baseline plasma cortisol levels compared with controls. In addition, the level of affective dysregulation was significantly negatively associated with citalopraminduced neuroendocrine responses. Neuroendocrine responses were however, not significantly attenuated in d-IBS patients compared with controls which may be explained by the fact that (i) we excluded patients with a previous psychiatric diagnosis or first-degree family history of affective disorders and (ii) the level of affective dysregulation in our IBS patients was less pronounced compared with patients primarily presenting with affective disorders or (iii) a type II error.

Our findings of increased plasma serotonergic and hormonal parameters and presence of an affective memory bias favour a peripheral as well as central influence of citalopram. It may be hypothesized that citalopram administration increases $5-\mathrm{HT}$ at the synapses. However, how this effects $5-\mathrm{HT}$ activity at target organ level (CNS and GI tract) remains uncertain in view of possible counter-regulatory mechanisms. Regarding citalopram pharmacokinetics Seifritz et al. reported that plasma citalopram metabolite concentrations were constant from the end of $20 \mathrm{mg}$ i.v. administration until 160 minutes afterwards ${ }^{40}$. Although we lack biochemical evidence of an increased serotonergic activity in the brain, the citalopram-induced alteration of affective memory performance indicate that central effects are apparent. Acute citalopram administration did not significantly decrease visceral perception. Although the pharmacokinetic and pharmacodynamic effect window do not 
need to be identical it is unlikely that during the assessment of visceral perception the pharmacodynamic effect was not yet present.

In our previous study lowering of the serotonergic synthesis induced a depression-like memory bias, i.e. induced a memory bias towards a loss of positive material and we hypothesized that this response bias maybe responsible for the induced enhanced visceral perception by affecting afferent visceral processing ${ }^{9}$. However, in the present study increasing the serotonergic synthesis induced a response bias towards positive material without an effect on visceral perception. Apparently, visceral perception is not directly associated with $5-\mathrm{HT}$ activity or response bias but probably is a more complex multifactor interaction.

The antidepressant effect of SSRIs in patients with major depression is known to occur after about 6 weeks of treatment. However, 6-week treatment with fluoxetine did not change rectal sensitivity in non-psychiatric IBS patients ${ }^{33}$. The present results concern the change in brain-gut interaction due to an acute increase of serotonergic activity. Whether these findings are also valid in a prolonged change of serotonergic activity by pharmacological or nutritional means and whether there is a differential effect in IBS with and without psychiatric comorbidity needs to be investigated.

The SERT polymorphism has been associated with vulnerability to stressinduced depressive symptoms and with the speed and rate of response to antidepressant treatment. In addition, SERT polymorphism has been associated with altered neuroendocrine and neuromodulatory responses following citalopram administration ${ }^{47}$. Also in IBS there is some evidence that genetic polymorphisms at the SERT promoter are associated with d-IBS and treatment response ${ }^{48}$. Wether functional responses of the serotonin system, may represent a neurobiological substrate for the differential response to antidepressant treatment in IBS needs to be elucidated.

We have provided evidence that acute serotonergic modulation by selective serotonin reuptake inhibition influences neuroendocrine responses and cognition in d-IBS and controls without a significant effect on visceral perception. 


\section{References}

1. Barbara G, De Giorgio R, Stanghellini V, Cremon C, Salvioli B, Corinaldesi R. New pathophysiological mechanisms in irritable bowel syndrome. Aliment Pharmacol Ther 2004; 20 Suppl 2:1-9.

2. Mayer EA, Gebhart GF. Basic and clinical aspects of visceral hyperalgesia. Gastroenterology 1994;107:271-293.

3. Vandvik PO, Wilhelmsen I, Ihlebaek C, Farup PG. Comorbidity of irritable bowel syndrome in general practice: a striking feature with clinical implications. Aliment Pharmacol Ther 2004; 20:1195-203.

4. Kilkens TO, Honig A, Rozendaal N, Van Nieuwenhoven MA, Brummer RJ. Serotonergic modulators in the treatment of irritable bowel syndrome - influence on psychiatric and gastrointestinal symptoms. Aliment Pharmacol Ther 2003;17:43-51.

5. Charney DS. Monoamine dysfunction and the pathophysiology and treatment of depression. J Clin Psychiatry 1998;59 Suppl 14:11-14.

6. Camilleri M. Serotonergic modulation of visceral sensation: lower gut. Gut 2002;51 Suppl 1: i81-86.

7. Houghton LA, Atkinson W, Whitaker RP, Whorwell PJ, Rimmer MJ. Increased platelet depleted plasma 5-hydroxytryptamine concentration following meal ingestion in symptomatic female subjects with diarrhoea predominant irritable bowel syndrome. Gut 2003;52:663-670.

8. Camilleri M, Northcutt AR, Kong S, Dukes GE, McSorley D, Mangel AW. Efficacy and safety of alosetron in women with irritable bowel syndrome: a randomised, placebo-controlled trial. Lancet 2000;355:1035-1040.

9. Kilkens TO, Honig A, van Nieuwenhoven MA, Riedel WJ, Brummer RJ. Acute tryptophan depletion affects brain-gut responses in irritable bowel syndrome patients and controls. Gut 2004;53:1794-1800.

10. Yatham LN, Steiner M. Neuroendocrine probes of serotonergic function: a critical review. Life Sci 1993;53:447-463

11. Hyttel J. Citalopram--pharmacological profile of a specific serotonin uptake inhibitor with antidepressant activity. Prog Neuropsychopharmacol Biol Psychiatry 1982;6:277-295.

12. Kapitany $T$, Schind $M$, Schindler SD, Hesselmann B, Fureder T, Barnas C, Sieghart W, Kasper $\mathrm{S}$. The citalopram challenge test in patients with major depression and in healthy controls. Psychiatry Res 1999;88:75-88.

13. Gorard DA, Dewsnap PA, Medbak SH, Perry LA, Libby GW, Farthing MJ. Central 5hydroxytryptaminergic function in irritable bowel syndrome. Scand J Gastroenterol 1995;30: 994-999.

14. Crowell MD. Role of serotonin in the pathophysiology of the irritable bowel syndrome. $\mathrm{Br} \mathrm{J}$ Pharmacol 2004;141:1285-1293.

15. Sheehan D. MINI International neuropsychiatric Interview: Tampa: University of South Florida; 1994

16. Hamilton M. Development of a rating scale for primary depressive illness. Br J Soc Clin Psychol 1967;6:278-296.

17. Arrindell WA, Ettema JHM. SCL-90. Een multidimensionale psychopathologie indicator $[S C L-$ 90. A multidimensional indicator of psychopathology]. In. Lisse, The Netherlands: Swets \& Zeitlinger; 1986.

18. Mykletun A, Stordal E, Dahl AA. Hospital Anxiety and Depression (HAD) scale: factor structure, item analyses and internal consistency in a large population. $\mathrm{Br} \mathrm{J}$ Psychiatry 2001; 179:540-544.

19. Luteijn F, van der Ploeg FAE. Handleiding Groninger Intelligentie test (GIT) [Manual Groninger Intelligence Test]. Lisse, the Netherlands: Swets and Zeitlinger; 1983.

20. Ledochowski $M$, Sperner-Unterweger B, Fuchs $D$. Lactose malabsorption is associated with early signs of mental depression in females: a preliminary report. Dig Dis Sci 1998;43: 2513-2517. 
21. Sobczak S, Riedel WJ, Booij I, Aan Het Rot M, Deutz NE, Honig A. Cognition following acute tryptophan depletion: difference between first-degree relatives of bipolar disorder patients and matched healthy control volunteers. Psychol Med 2002;32:503-515.

22. Drossman DA, Heaton K, Irvine EJ, Muller-Lissner S. Functional bowel disorders and functional abdominal pain. In: Drossman DA, Corazziari $E$, Talley N, Thompson WG, Whitehead WE, editors. The functional gastrointestinal disorders. Diagnosis, pathophysiology and treatment: A multinational consensus. McLean, VA: Degnon Associates; 2000:351-398.

23. Houghton LA, Lea R, Jackson N, Whorwell PJ. The menstrual cycle affects rectal sensitivity in patients with irritable bowel syndrome but not healthy volunteers. Gut 2002;50:471-474.

24. Riedel WJ, Klaassen T, Deutz NE, van Someren A, van Praag HM. Tryptophan depletion in normal volunteers produces selective impairment in memory consolidation. Psychopharmacology (Berl) 1999;141:362-369.

25. Mcnair DM, Lorr M, Droppleman LF. Manual for the Profile of Mood States (POMS) manual. Educational and Industrial Testing Service. San Diego, CA; 1992.

26. Stronks DL, Tulen JH, Verheij R, Boomsma F, Fekkes D, Pepplinkhuizen L, Mantel GW, Passchier J. Serotonergic, catecholaminergic, and cardiovascular reactions to mental stress in female migraine patients. A controlled study. Headache 1998;38:270-280

27. Fekkes D, Timmerman L, Pepplinkhuizen L. Effects of clomipramine on plasma amino acids and serotonergic parameters in panic disorder and depression. Eur Neuropsychopharmacol 1997; 7:235-239.

28. Riedel WJ, Klaassen T, Griez E, Honig A, Menheere PP, van Praag HM. Dissociable hormonal, cognitive and mood responses to neuroendocrine challenge: evidence for receptor-specific serotonergic dysregulation in depressed mood. Neuropsychopharmacology 2002;26:358-367.

29. Tack J, Sarnelli G. Serotonergic modulation of visceral sensation: upper gastrointestinal tract. Gut 2002;51 Suppl 1:i77-80.

30. Broekaert D, Vos R, Gevers A, Janssens J, Vandenberghe J, Fischler B, Tack J. A doubleblind randomised placebo-controled crossover trial of citalopram, a selective serotonin reuptake inhibitor, in irritable bowel syndrome. Gastroenterology 2001;120:A641.

31. Tack JF, Vos R, Broeckaert D, Fischler B, Janssens B. Influence of citalopram, a selective serotonin reuptake inhibitor, on colonic tone and sensitivity in man. Gastroenterology 2000; 118:A175.

32. Siproudhis $L$, Dinasquet $M$, Sebille $V$, Reymann $J M$, Bellissant $E$. Differential effects of two types of antidepressants, amitriptyline and fluoxetine, on anorectal motility and visceral perception. Aliment Pharmacol Ther 2004;20:689-695.

33. Kuiken SD, Tytgat GN, Boeckxstaens GE. The selective serotonin reuptake inhibitor fluoxetine does not change rectal sensitivity and symptoms in patients with irritable bowel syndrome: a double blind, randomized, placebo-controlled study. Clin Gastroenterol Hepatol 2003;1:219-228.

34. Steens J, Van Der Schaar PJ, Penning C, Brussee J, Masclee AA. Compliance, tone and sensitivity of the rectum in different subtypes of irritable bowel syndrome. Neurogastroenterol Motil 2002;14:241-247.

35. Harmer CJ, Bhagwagar Z, Cowen PJ, Goodwin GM. Acute administration of citalopram facilitates memory consolidation in healthy volunteers. Psychopharmacology (Berl) 2002; 163:106-110.

36. Harmer CJ, Bhagwagar Z, Perrett DI, Vollm BA, Cowen PJ, Goodwin GM. Acute SSRI administration affects the processing of social cues in healthy volunteers. Neuropsychopharmacology 2003;28:148-152.

37. Harmer CJ, Shelley NC, Cowen PJ, Goodwin GM. Increased positive versus negative affective perception and memory in healthy volunteers following selective serotonin and norepinephrine reuptake inhibition. Am J Psychiatry 2004;161:1256-1263.

38. Kemp AH, Gray MA, Silberstein RB, Armstrong SM, Nathan PJ. Augmentation of serotonin enhances pleasant and suppresses unpleasant cortical electrophysiological responses to visual emotional stimuli in humans. Neuroimage 2004;22:1084-1096. 
142 Chapter?

39. Paffen MLJE, Kilkens TOC, Honig A, Brummer R-JM, Van Nieuwenhoven MA. Does a rectal barostatprocedure induce stress in IBS patients and healthy controls? Eur J Gastroenterol Hepatol 2003;15:A38.

40. Seifritz E, Baumann $P$, Muller MJ, Annen $O$, Amey $M$, Hemmeter $U$, Hatzinger $M$, Chardon $F$, Holsboer-Trachsler $E$. Neuroendocrine effects of a $20-\mathrm{mg}$ citalopram infusion in healthy males. A placebo-controlled evaluation of citalopram as 5-HT function probe. Neuropsychopharmacology 1996;14:253-263.

41. Lotrich FE, Bies R, Muldoon MF, Manuck SB, Smith GS, Pollock BG. Neuroendocrine response to intravenous citalopram in healthy control subjects: pharmacokinetic influences. Psychopharmacology (Berf) 2005;178:268-275.

42. Henning $J$, Netter $P$. Oral application of citalopram (20 mg) and its usefulness for neuroendocrine challenge tests. Int J Neuropsychopharmacol 2002;5:67-71.

43. Nadeem HS, Attenburrow MJ, Cowen PJ. Comparison of the Effects of Citalopram and Escitalopram on 5-Ht-Mediated Neuroendocrine Responses. Neuropsychopharmacology 2004.

44. Heitkemper $M$, Jarrett $M$, Cain $K$, Shaver J, Bond E, Woods NF, Walker E. Increased urine catecholamines and cortisol in women with irritable bowel syndrome. Am J Gastroenterol 1996;91:906-913.

45. Patacchioli FR, Angelucci L, Dellerba G, Monnazzi P, Leri O. Actual stress, psychopathology and salivary cortisol levels in the irritable bowel syndrome (IBS). J Endocrinol Invest 2001; 24:173-177.

46. Steckler T, Holsboer F, Reul JM. Glucocorticoids and depression. Baillieres Best Pract Res Clin Endocrinol Metab 1999;13:597-614.

47. Eichhammer $P$, Langguth $B$, Wiegand $R$, Kharraz $A$, Frick $U$, Hajak $G$. Allelic variation in the serotonin transporter promoter affects neuromodulatory effects of a selective serotonin transporter reuptake inhibitor (SSRI). Psychopharmacology (Berl) 2003;166:294-297.

48. Yeo A, Boyd $P$, Lumsden $S$, Saunders $T$, Handley $A$, Stubbins $M$, Knaggs A, Asquith $S$, Taylor I, Bahari B, Crocker N, Rallan R, Varsani S, Montgomery D, Alpers DH, Dukes GE, Purvis I, Hicks GA. Association between a functional polymorphism in the serotonin transporter gene and diarrhoea predominant irritable bowel syndrome in women. Gut 2004; $53: 1452-1458$ 


\section{Chapter 8}

Rectal compliance and neurohormonal-immune signalling in diarrhoea-predominant irritable bowel syndrome

Kilkens TO, van Nieuwenhoven MA, Brummer RJ. 
144 Chapter

\section{Abstract}

\section{Background}

Irritable bowel syndrome (IBS) is regarded as a disorder of brain-gut axis regulation. Abnormal rectal compliance seems a consistent feature of diarrhoea-predominant (d-)!BS. The serotonergic system, HPA-axis, and immune system are all integrated within the brain-gut concept.

Aim

The aim of this study was to investigate differences in rectal compliance and its relationship to biochemical parameters of serotonergic metabolism, HPA axis and immune activation, respectively, in patients with $d-I B S$ and healthy controls.

Methods

(i) Platelet poor plasma 5-hydroxytryptamine (ppp 5-HT), its metabolite 5-hydroxyindole acetic acid (5-HIAA), 5-HT turnover (5-HIAA:5-HT), platelet 5-HT, (ii) salivary cortisol and, (iii) serum levels of the proinflammatory cytokine TNF- $a$ were used as parameters of serotonergic metabolism, HPA axis and immune activation, respectively, in 22 d-IBS patients and 22 matched healthy controls. Rectal compliance (pressure-volume relationship) was assessed using a barostat procedure. Subsequently, group differences and associations between the biochemical parameters and rectal compliance were analysed.

Results

The d-IBS patients showed significantly increased ppp 5 -HT levels $(p=0.03)$, decreased 5-HIAA levels $(p=0.03)$, decreased 5 -HT turnover $(p=0.04)$ and reduced rectal compliance $(p=0.002)$ compared to controls. The levels of ppp $5-H T(p=0.03)$, cortisol $(p=0.02)$ and TNF- $\alpha(p=0.02)$ correlated significantly with reduced rectal compliance. Ppp 5-HT was significantly positively associated with cortisol levels $(p=0.004)$.

\section{Conclusion}

We demonstrated that reduced rectal compliance in d-IBS is significantly related to alterations in neurohormonal and immune signalling. 
Rectal compliance and neurohormonal-immune signalling $\mid 145$

\section{Introduction}

Irritable bowel syndrome (IBS) is conventionally regarded as a symptom complex characterized by abdominal pain or discomfort associated with alteration in defecation, in the absence of structural or biochemical abnormalities ${ }^{1}$. Approximately $10-20 \%$ of the Western population has symptoms consistent with a diagnosis of IBS and IBS is associated with a significant impairment in quality of life $^{2}$. Although altered intestinal motor physiology and visceral perception contribute to the symptom generation of IBS the pathophysiology of IBS is not fully understood ${ }^{1}$. A multicomponent conceptual model of IBS has been postulated, involving physiologic, affective, cognitive and behavioural factors. The brain-gut axis consists of communication pathways connecting the central nervous system (CNS) and the enteric nervous system (ENS) with neuroendocrine centres, and the immune system ${ }^{3}$. IBS is now generally recognized as a disorder involving the brain-gut axis.

Serotonin $(5-\mathrm{HT})$ is an important neurotransmitter and paracrine signalling molecule and is involved in a number of physiologic functions, including mood, appetite, emesis, sleep, memory and learning, homeostasis and sexual behaviours. The major source of bioavailable $5-\mathrm{HT}$ is located in the gastrointestinal $(\mathrm{Gl})$ tract. In the $\mathrm{GI}$ tract $5-\mathrm{HT}$ regulates secretion, motility, vasodilatory reflexes and perception. Hence, serotonin is considered one of the key denominators of the brain-gut axis ${ }^{4}$. Modulation of serotonergic activity is currently under investigation in the treatment of IBS ${ }^{5-7}$. Recent observations suggest that serotonergic dysregulation may be specifically relevant in the diarrhoea-predominant type of IBS (d-IBS), although the role and the nature of disturbed serotonergic metabolism in the pathophysiology of d-IBS are not fully understood ${ }^{8,9}$.

Alterations in the hypothalamic-pituitary-adrenal (HPA) axis function also play an important role in brain-gut interactions and therefore in the pathophysiology of IBS. IBS has been associated with altered levels of the stress hormone cortisol $^{10,11}$. There is increasing evidence that links $5-\mathrm{HT}$ with HPA regulation ${ }^{12}$. IBS has been characterized by an overactivation of the HPA axis, a proinflammatory cytokine increase, and low-grade inflammation has been postulated as a basis for alterations in intestinal motility or sensation ${ }^{13-15}$. In addition, both the HPA axis and serotonergic mechanisms are involved in the immune response by modulating the production of cytokines ${ }^{12}$.

Reduced rectal compliance may be a consistent feature of $d-\mid B S^{16,17}$. We previously demonstrated that rectal compliance was significantly reduced in $d$ - 
146 Chapter 8

IBS patients and that acute serotonergic challenge did not significantly affect rectal compliance in d-IBS patients or healthy controls ${ }^{18,19}$. However, the relationship of basal (i.e. unchallenged) biochemical parameters of serotonergic metabolism, HPA axis and immune activation with rectal compliance is unknown. Therefore this study investigates: (i) rectal compliance, (ii) platelet poor plasma (ppp) 5-HT, its metabolite 5-hydroxyindole acetic acid (5-HIAA), 5-HT turnover (5-HIAA:5-HT) and platelet 5-HT, respectively, as parameters of $5-\mathrm{HT}$ metabolism, and salivary cortisol and serum levels of tumour necrosis factor-alpha (TNF- $\alpha$ ) as parameters of HPA axis and immune activation, respectively, and (iii) the relationship between these parameters and rectal compliance in a homogenous group of d-IBS patients and matched healthy controls.

\section{Materials and methods}

\section{Subjects}

The Medical Ethics Committee of the University Hospital Maastricht approved the study protocol and all subjects gave written informed consent before the start of the study. The study was carried out on 22 patients and 22 matched control subjects. The patients fulfilled the diagnosis of d-IBS according to the ROME II criteria, as diagnosed by an experienced gastroenterologist ${ }^{20}$. Exclusion criteria were abdominal surgery (other than appendectomy and cholecystectomy), use of medications other than oral contraceptives within fourteen days prior to testing, lactose malabsorption ${ }^{21}$, presence of an acute infection episode, any history of psychiatric disease or use of psychoactive medication, premenstrual syndrome, dieting, pregnancy or lactation, excessive alcohol intake ( $>20$ alcoholic units a week), and hypertension (diastolic $>100$ $\mathrm{mmHg}$, systolic $>170 \mathrm{mmHg}$ ), respectively. During the screening procedure general psychological state was assessed using the 17-item Hamilton Depression Rating Scale (HAM-D17) ${ }^{22}$, the Dutch version of the Symptom Check List $(\mathrm{SCL}-90)^{23}$ and the Hospital Anxiety and Depression Scale $(\mathrm{HADS})^{24}$. Physical health was assessed by means of a standard physical examination and a urine test. A pregnancy test was performed in all female subjects. The healthy control subjects were carefully matched for age, gender, Body Mass Index (BMI), use of oral contraceptives ${ }^{25,26}$. The same exclusion criteria as those for the patients were applied. Additional exclusion criteria for the control subjects were current or history of a Gl disorder, current psychiatric or psychological symptomatology defined as: HAM-D17 scores above 18, global severity index score on the SCL-90 for females $\geq 150$ and for males $\geq 131$, or HADS scores $\geq 8$. All women were tested in the follicular phase of the 
menstrual cycle or while taking oral contraception ${ }^{27}$. To avoid possible seasonal variation all subjects were tested within three months time ${ }^{28}$. They were asked to abstain from heavy physical exercise and consumption of alcoholic beverages the day prior to their visit.

\section{Study design}

The subjects attended the laboratory after an overnight fast (after 10:00 PM eating, smoking or drinking was not allowed) at 8:00 AM. Saliva was collected for cortisol measurement. Subsequently, venous blood was obtained for determination of serotonergic and immune parameters. Thereafter, rectal compliance was assessed using a rectal barostat procedure.

\section{Biochemical parameters}

Concentrations of platelet poor plasma (ppp) 5-HT, its major metabolite 5HIAA, 5-HT turnover (5-HIAA:5-HT) and platelet 5-HT were determined as markers of 5-HT metabolism. Blood was sampled in EDTA Vacutainer tubes and kept at room temperature. For platelet 5-HT measurement, whole blood was used. In addition, an aliquot of blood was used for whole blood platelet counting. Samples for ppp 5-HT and 5-HIAA were centrifuged (20 minutes, $2600 \mathrm{~g}, 20^{\circ} \mathrm{C}$ ). As a control of the ppp preparation procedure, platelets were also counted in the platelet poor plasma using a Coulter Counter (Coulter MD Series, Coulter Corporation, Hialeah, Florida, USA). All samples were stored at $-80^{\circ} \mathrm{C}$ until analysis. Platelet poor plasma 5-HT, 5-HIAA and platelet 5-HT samples were analysed using high performance liquid chromatography (HPLC), as previously described ${ }^{29-31}$. Saliva collection was performed using cotton Salivettes (Sarstedt, the Netherlands) ${ }^{32}$. Uncentrifuged samples were stored at $-20^{\circ} \mathrm{C}$ until analysis. Cortisol levels were determined in duplicate in $50 \mu \mathrm{l}$ saliva by direct radio-immunoassay, using an HPLC-purified preparation of cortisol-3CMO-histamine ${ }^{125} \mathrm{l}^{33}$. The lower detection limit of the assay was $11.9 \mathrm{ng} / \mathrm{dl}$. Blood for serum TNF- $\alpha$ was sampled in Vacutainer (SST) tubes. Samples were centrifuged $\left(10\right.$ minutes, $\left.900 \mathrm{~g}, 4^{\circ} \mathrm{C}\right)$ and stored at $-80^{\circ} \mathrm{C}$ until analysis. Serum concentrations of TNF-a were measured using a commercially available enzyme-linked immunosorbent assay (ELISA)(Bender MedSystems, Vienna, Austria). The assay was performed according manufacturer instructions. The detection limit in our laboratory was $8 \mathrm{pg} / \mathrm{ml}$.

\section{Barostat procedure}

The bowel preparation consisted of a tap water enema one hour before the barostat procedure. The rectal probe was lubricated and placed into the rectum so that the attached end of the bag was $3 \mathrm{~cm}$ from the external anal sphincter. 
$148 \mid$ Chapter 8

The probe consisted of a $700 \mathrm{ml}$ polyethylene bag secured on a rectal catheter (external diameter $=18$ French). The catheter was connected to the electronic distension device (Electronic Barostat; Distender Series II; G \& J Electronics Inc., Toronto, Ontario, Canada) using the Protocol Plus Deluxe Software ( $G$ \& J Electronics Inc.). The barostat inflation rate was $50 \mathrm{ml} / \mathrm{s}$ to a constant pressure plateau. To reduce the influence of adipose tissue mass and abdominal wall tone, subjects were all placed in the left lateral position. In order to unfold the rectal probe, a five minute $10 \mathrm{mmHg}$ distension followed by one minute $20 \mathrm{mmHg}$ distension was administered. Subsequently, the standardized barostat protocol consisted of intermittent distensions of 60 seconds duration at pressures $10-70 \mathrm{mmHg}$, separated by an interval of 30 seconds baseline pressure $(0 \mathrm{mmHg})$. The endpoint to stop the series of distensions was the perceptual threshold for maximal tolerable discomfort or if a maximal volume of $600 \mathrm{ml}$ or pressure $70 \mathrm{mmHg}$ was reached ${ }^{19}$. As the occurrence of pain may reflect the point where the adaptability of the rectal wall is reaching its limits we chose to analyse rectal compliance within the physiologic distension range of the gut, i.e. up to $25 \mathrm{mmHg}$, a pressure which induces minor to normal urge to defecate ${ }^{18,19}$. Apparent dynamic rectal compliance $(\Delta \mathrm{V} / \Delta \mathrm{P} ; \mathrm{ml} / \mathrm{mmHg})$ was determined by calculating the difference in rectal volume (rectal volume Pressure $25_{2}$ - rectal volume pressure 10) and divided by the difference in rectal pressure $(15 \mathrm{mmHg})$.

\section{Statistical analysis}

According to the Shapiro-Wilks normality tests subject characteristics and outcome parameters were normally distributed and therefore independent samples $t$-tests were used. For the multiple biochemical $5-\mathrm{HT}$ parameters a multivariate ANOVA was used. Associations between biochemical outcome parameters and between biochemical outcome parameters with rectal compliance were analysed by linear regression in the entire study population. Cook's distance was used to identify possible influential cases according to the lines described by Hair et al. ${ }^{34}$. A two-tailed $p$-value $<0.05$ was considered statistically significant. Data are presented as mean (SE). Statistical analyses were performed using the SPSS 12.0 for Windows software package.

\section{Results}

\section{Sample description}

Table 8.1 summarizes the characteristics of the 44 participants $(22 \mathrm{~d}$-IBS patient and 22 healthy controls). Subjects were successfully matched with 
regard to sex, age, BMl and contraceptive use. In addition, there were no significant differences in alcohol and cigarette consumption between patients and controls. Severity of affective dysregulation, as indicated by the SCL, HADS, and HAM-D17 scores, was significantly higher in d-IBS patients compared with healthy controls $(p \leq 0.01)$.

Table 8.1 Subject characteristics (mean (SE)).

\begin{tabular}{lccc}
\hline & $\begin{array}{c}\text { d-IBS patients } \\
n=22\end{array}$ & $\begin{array}{c}\text { Controls } \\
n=22\end{array}$ & $\begin{array}{c}\text { Patients vs. Controls } \\
p \text { value }\end{array}$ \\
\hline Age & $33.4(2.4)$ & $33.0(2.7)$ & 0.9 \\
Females $(n)$ & $13 / 22$ & $13 / 22$ & - \\
Oral contraceptives $(\mathrm{n})$ & $11 / 13$ & $11 / 13$ & - \\
Body mass index & $23.2(0.9)$ & $22.8(0.5)$ & 0.7 \\
Alcohol units/day & $0.58(0.2)$ & $0.54(0.1)$ & 0.8 \\
Cigarettes/day & $2.2(0.8)$ & $0.9(0.6)$ & 0.2 \\
HAM-D17 & $4.5(0.9)$ & $1.0(0.3)$ & $0.001^{\mathrm{b}}$ \\
SCL-90 (GSI) & $116(4.9)$ & $97.7(2.2)$ & $0.001^{\mathrm{b}}$ \\
HADS (total) & $6.0(0.6)$ & $3.5(0.7)$ & $0.01^{\mathrm{b}}$ \\
\hline
\end{tabular}

${ }^{a}$ Independent-samples $t$-tests comparing d-IBS patients versus control subjects

b Significant difference between groups. d-IBS; diarrhoea-predominant irritable bowel syndrome; HAM-D17, 17-item Hamilton Depression Rating Scale; SCL-90, Symptom Check List; GSI, Global Severity Index; HADS, Hospital Anxiety and Depression Scale.

\section{Group differences in outcome parameters}

Multivariate analysis showed a significant difference in 5-HT parameters between $d$-IBS patients and healthy controls $\left(F_{(\mathrm{HOT})}=3.27, d f=4, p=0.02\right)$. Subsequent univariate analyses revealed statistical significances of increased ppp 5-HT $(p=0.03)$, decreased 5-HIAA $(p=0.03)$ and decreased 5-HT turnover $(p=0.04)$, in the patient compared with the control group. Platelet 5-HT concentrations did not significantly differ between the patient and the control group ( $p=0.4$ ). The cortisol concentrations tended to be increased in the patient compared with the control group, however this difference did not reach significance $(p=0.1)$. TNF- $\alpha$ levels did not significantly differ between the patient and the control group ( $p=0.2)$ (Table 8.2). Rectal compliance was significantly decreased in d-IBS patients compared with the healthy controls $(p=0.002)$ (Figure 8.1), confirming the presence of reduced rectal compliance in this $d$-IBS subgroup. 


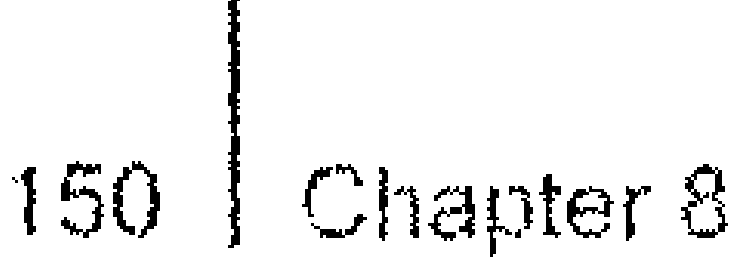

Table 8.2 Biochemical parameters (mean (SE)) in the diarrhoea-predominant irritable bowel syndrome (d-IBS) patients and control subjects.

\begin{tabular}{lccc}
\hline & $\begin{array}{c}\text { d-IBS patients } \\
n=22\end{array}$ & $\begin{array}{c}\text { Controls } \\
n=22\end{array}$ & $\begin{array}{c}\text { Patients vs. Controls } \\
\text { ppp value }\end{array}$ \\
ppp 5-HT (nmol/l) & $11.8(1.3)$ & $8.2(0.8)$ & $0.03^{\mathrm{a}}$ \\
5-HT turnover & $29.0(1.0)$ & $32.9(1.5)$ & $0.03^{\mathrm{a}}$ \\
platelet 5-HT (nmol/10 platelets) & $3.45(0.5)$ & $4.88(0.5)$ & $0.04^{\mathrm{a}}$ \\
cortisol (ng/d) & $3.91(0.3)$ & $4.32(0.3)$ & $0.36^{\mathrm{a}}$ \\
TNF-a (pg/ml) & $621(49)$ & $503(52)$ & $0.10^{b}$ \\
\hline
\end{tabular}

${ }^{a}$ Multivariate analysis indicated a significant difference in multiple 5-HT parameters comparing dIBS patients versus control subjects, in this table univariate $p$ values are shown.

${ }^{b}$ Independent-samples $t$-tests comparing $d-\{B S$ patients versus control subjects.

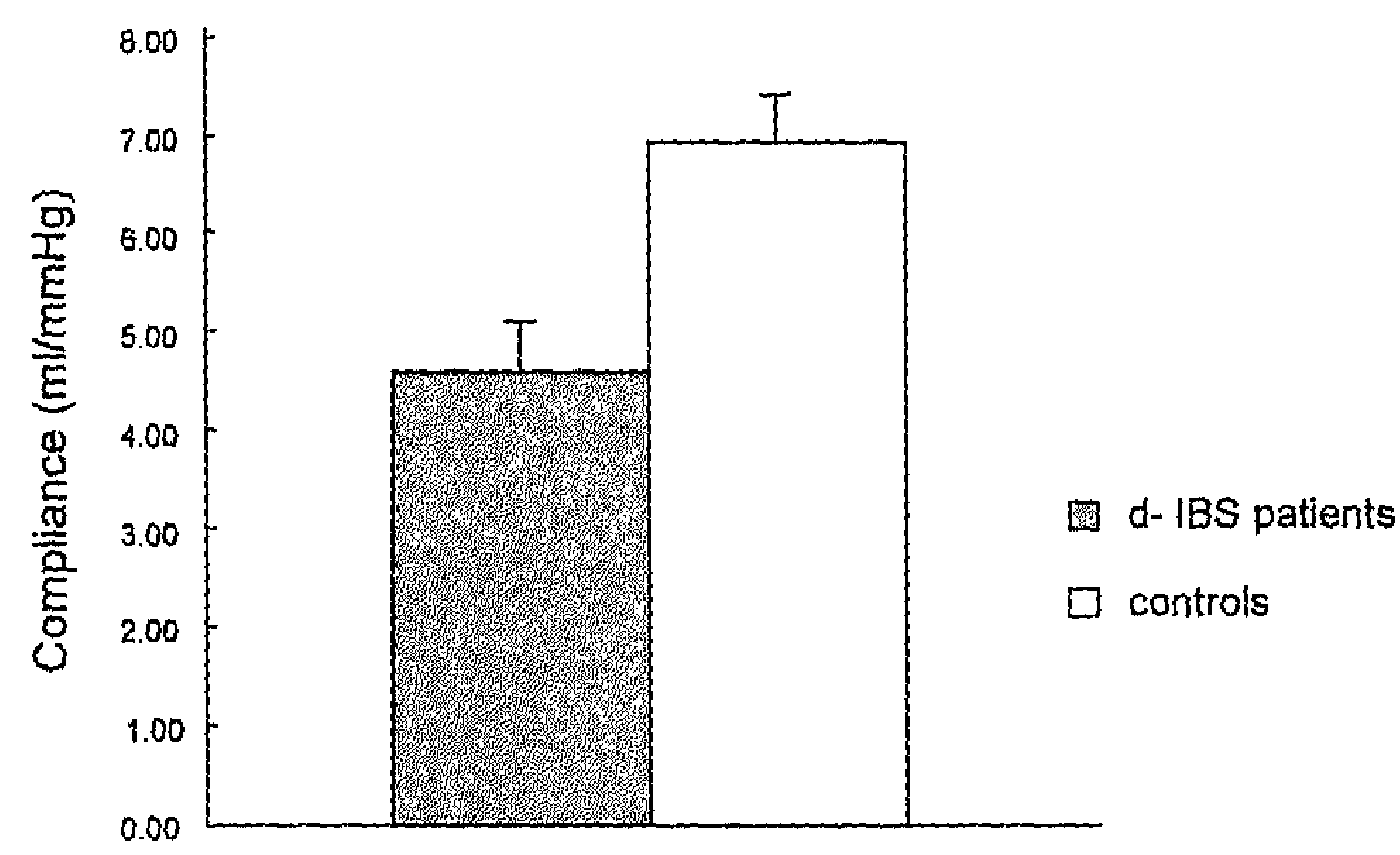

Figure 8.1 Rectal compliance in diarrhoea-predominant irritable bowel syndrome patients (d-IBS) and healthy controls (mean (SE)). Patients had decreased rectal compliance compared with controls $(p=0.002)$.

\section{Associations between biochemical parameters and rectal compliance}

Ppp 5-HT was significantly negatively associated with rectal compliance $\left(F=5.2, p=0.03, R^{2}=0.11\right)$ (Figure 8.2$)$. There was no significant association between 5-HIAA ( $F=0.009, p=0.92, R^{2}=0.000$ ) and platelet 5 -HT ( $F=0.01$, $p=0.94, R^{2}=0.000$ ) with rectal compliance. The $5-H T$ turnover was significantly positively associated with rectal compliance $\left(F=4.5, p=0.04, R^{2}=0.10\right)$. Both the cortisol $\left(F=5.9, p=0.02, R^{2}=0.12\right)$ and TNF-a concentrations $(F=5.5, p=0.02$, $R^{2}=0.12$ ) were significantly negatively associated with rectal compliance (Figures 8.3, 8.4). 


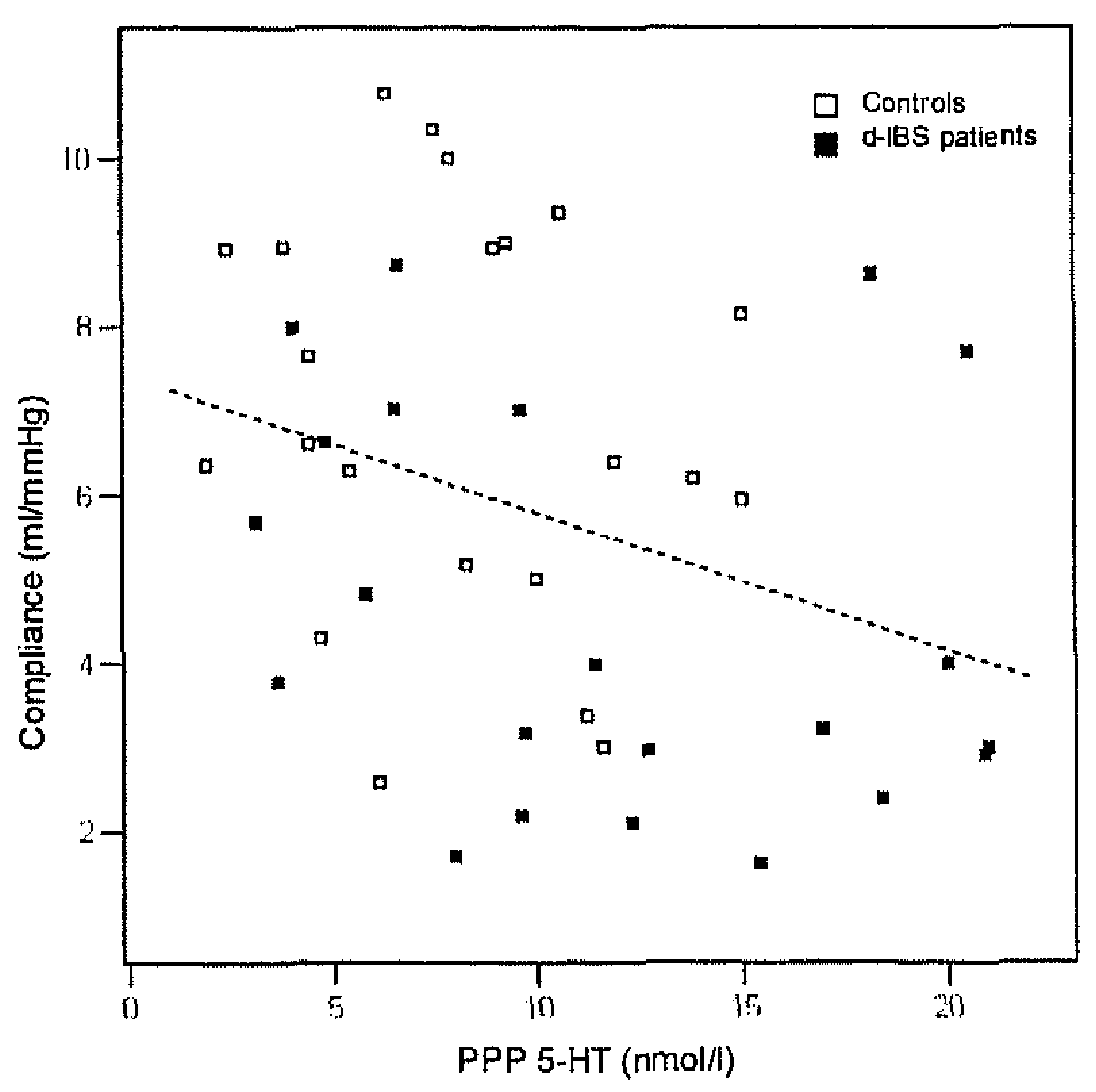

Figure 8.2 Association between platelet poor plasma (ppp) $5-H T$ and rectal compliance in the entire study population. Ppp 5-HT was significantly negatively associated with rectal compliance $(p=0.03)$.

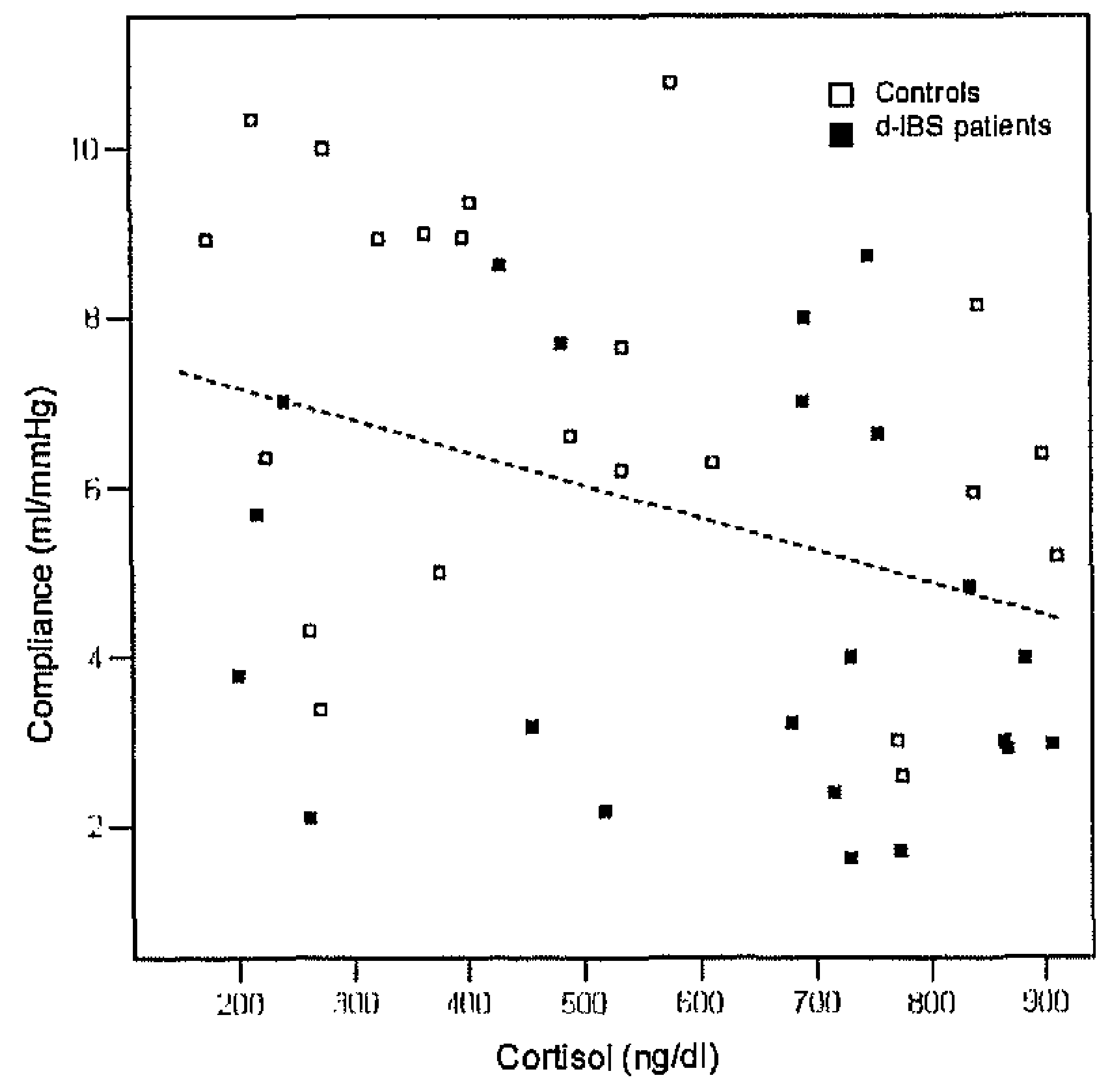

Figure 8.3 Association between salivary cortisol concentrations and rectal compliance in the entire study population. The cortisol concentrations were significantly negatively associated with rectal compliance $(p=0.02)$. 


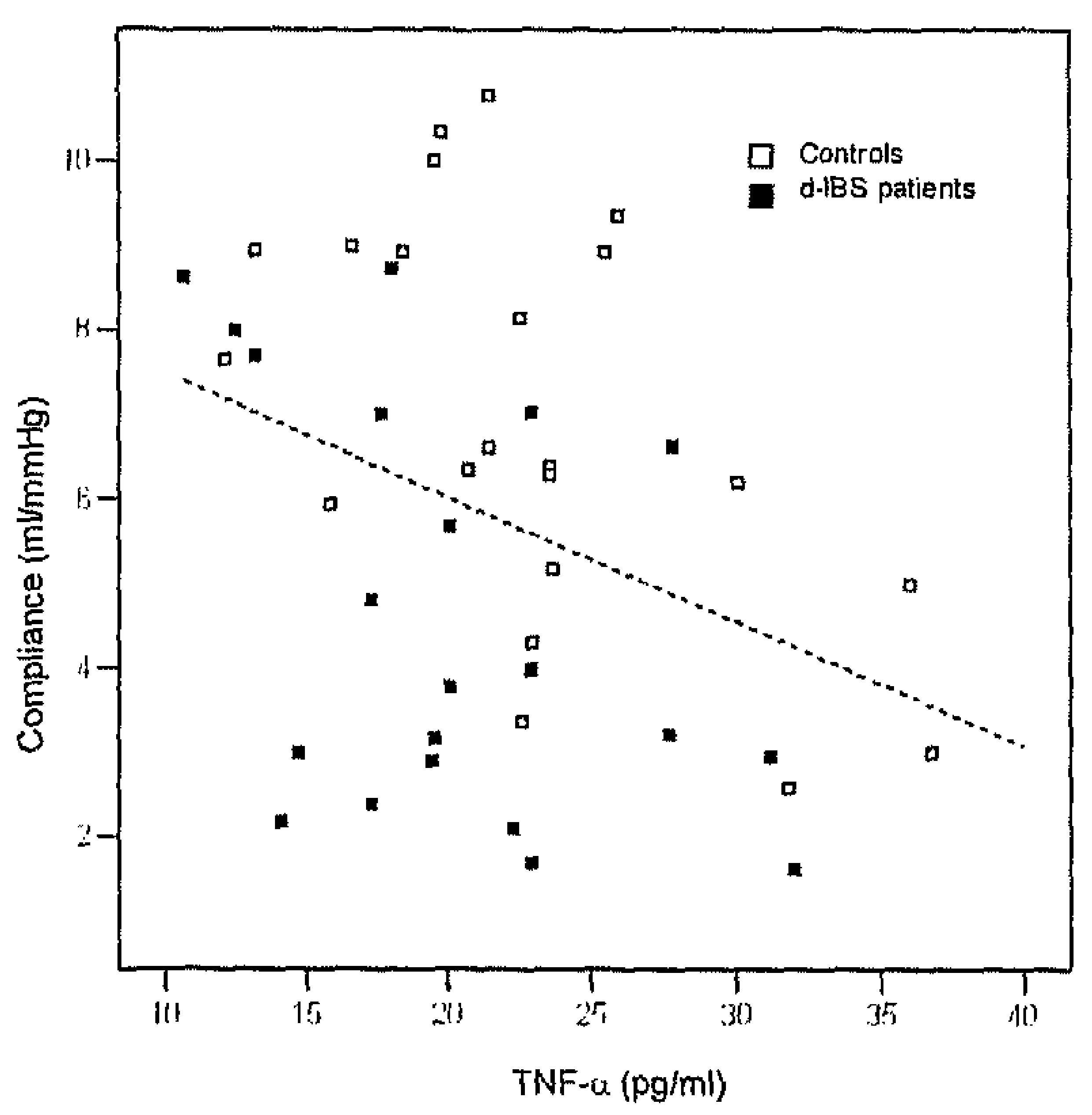

Figure 8.4 Association between serum TNF-a levels and rectal compliance in the entire study population. The TNF-a levels were significantly negatively associated with rectal compliance $(p=0.02)$.

\section{Associations between biochemical parameters}

Cortisol concentrations were significantly positively associated with ppp 5-HT $\left(F=9.4, p=0.004, R^{2}=0.18\right)$ but not with ppp 5-HIAA ( $\left.F=0.12, p=0.73, R^{2}=0.003\right)$ or platelet $5-\mathrm{HT}\left(\mathrm{F}=0.93, p=0.34, \mathrm{R}^{2}=0.02\right)$, respectively. $5-\mathrm{HT}$ turnover was significantly negatively associated with cortisol concentrations $(F=7.9, p=0.007$, $\left.R^{2}=0.16\right)$. TNF- $\alpha$ levels were not significantly associated with $5-H T$ parameters or cortisol levels $(p>0.4)$.

\section{Discussion}

This is the first study to show that rectal compliance in a study population of diarrhoea-predominant type of IBS (d-IBS) and healthy controls is related to parameters of neurohormonal-immune signalling as indicated by the relationship of platelet poor plasma 5-hydroxytryptamine (ppp 5-HT), salivary cortisol and serum TNF- $\alpha$ levels with reduced rectal compliance. In addition, ppp 5-HT levels were significantly positively correlated with cortisol levels. Furthermore, these results confirm not only the presence of reduced rectal compliance but also of increased ppp 5-HT levels in patients with d-IBS ${ }^{16-19}$.

The findings of raised ppp 5-HT and decreased 5-HT turnover with no change in platelet 5-HT concentrations under fasting conditions in d-IBS are in 
accordance with a recent study aiming to measure $5-\mathrm{HT}$ parameters under fasting and fed conditions in d-IBS, constipation predominant (C-)IBS patients, and healthy controls, respectively ${ }^{8}$. In contrast to this study, which also reported decreased 5-HIAA levels in c-IBS compared with d-IBS and healthy controls, we demonstrated significantly decreased ppp 5-HIAA in d-IBS patients ${ }^{8}$. The increased ppp 5-HT levels in d-IBS may be associated with decreased mucosal serotonin reuptake transporter (SERT) protein expression, which plays a pivotal role in the termination of action of 5-HT in the mucosa, and reduced 5-HT reuptake ${ }^{8,9,35}$. Interestingly, inflammation of the mucosa is generally considered to be associated with a decrease in the expression of SERT, which is supported by recent data that TNF- $\alpha$ modulates SERT activity $^{36-38}$. Apparently, raised ppp 5-HT levels in d-IBS are a consistent finding whereas results concerning 5-HIAA in various IBS subgroups are not. Hence, the precise mechanisms underlying the altered serotonergic signalling in d-IBS and other clinically relevant IBS subgroups needs further study.

Ppp 5-HT levels under fasting conditions were significantly associated with rectal compliance. Findings on colonic and rectal compliance in response to 5 -HT modulation are inconsistent ${ }^{5,39-42}$. We previously demonstrated that acute serotonergic modulation did not affect rectal compliance in d-IBS or controls ${ }^{18,19}$. These apparent equivocal findings may be explained by the fact that changes in serotonergic signalling achieved by pharmacological intervention are not directly comparable with the effects of unchallenged biological variation in serotonergic signalling on rectal compliance. This might be reflected by the observation that rectal compliance was not only associated with the unchallenged biological variation in $5-\mathrm{HT}$ signalling but also associated with variation in HPA axis and immune activation.

The negative association between cortisol levels and rectal compliance may be mediated by the autonomic nervous system (ANS) or potentially achieved by a direct effect on smooth muscle cell reactivity ${ }^{43,44}$. Some evidence exists for increased basal HPA axis activation in IBS as indicated by increased cortisol levels ${ }^{10,11,13,45}$. The cortisol concentrations tended to be increased in the patient compared with the control group. Failure of reaching statistical significance may be due to a type II error.

We chose to carefully match our group of d-IBS patients and healthy controls for potential confounding factors by matching them for age, gender, BMI, smoking behaviour and use of oral contraceptives ${ }^{46,47}$. The potential influence of anticipatory anxiety cannot be eliminated as biochemical assessment and the barostat procedure were conducted on the same test day ${ }^{48,49}$. 
154 Chapter 8

Although it is known that stimulation of serotonergic neurotransmission induces a hypothalamic-pituitary-adrenal axis-mediated neuroendocrine response, we are not aware of any other reports concerning the relationship between basal ppp 5-HT and salivary cortisol levels ${ }^{50}$. The positive association between these biomarkers of brain-gut dysregulation could have potential value regarding future brain-gut interaction research.

TNF-a was significantly negatively associated with rectal compliance. This may fit observations that TNF- $\alpha$ contributes to motility dysfunction and the findings that IBS has been associated with the high producer TNF- $\alpha$ genotype ${ }^{51,52}$. Furthermore, IBS patients failed to show a postprandial decrease in the in vitro TNF-a production ${ }^{53}$.

The clear association between neurohormonal-immune parameters and rectal compliance in the entire study population strengthens the direct biological association between these parameters, rather than the existence of a dominant disease modifying factor specific for IBS. We studied parameters at baseline under fasting conditions. However, whether these associations are also present in a postprandial state remains to be elucidated and future studies may also take into account the potential interaction with the ANS and noradrenergic pathways, which are intimately connected with the brain-gut axis ${ }^{54,55}$. In addition, these data do not provide clear evidence for a causal relationship between mechanisms of neurohormonal-immune signalling and rectal compliance. This necessitates other mechanistic studies.

We demonstrated that reduced rectal compliance in d-IBS is associated with alterations in neurohormonal and immune signalling. This study may facilitate the search for biomarkers of integrated brain-gut function in IBS. 


\section{References}

1. Talley NJ, Spiller R. Irritable bowel syndrome: a little understood organic bowel disease? Lancet 2002;360:555-564.

2. Hungin AP, Whorwell PJ, Tack J, Mearin F. The prevalence, patterns and impact of irritable bowel syndrome: an international survey of 40,000 subjects. Aliment Pharmacol Ther 2003; 17:643-550.

3. Drossman DA, Camilleri M, Mayer EA, Whitehead WE. AGA technical review on irritable bowel syndrome. Gastroenterology 2002;123:2108-2131.

4. Kim DY, Camilleri M. Serotonin: a mediator of the brain-gut connection. Am J Gastroenterol 2000;95:2698-2709.

5. Tack J, Broekaert D, Fischler B, Van Oudenhove L, Gevers A, Janssens J. A controlled cross-over study of the selective serotonin reuptake inhibitor citalopram in irritable bowe syndrome. Gut 2006.

6. Jackson JL, O'Malley PG, Tomkins G, Balden E, Santoro J, Kroenke K. Treatment of functional gastrointestinal disorders with antidepressant medications: a meta-analysis. Am J Med 2000;108:65-72.

7. Kilkens TO, Honig A, Rozendaal N, Van Nieuwenhoven MA, Brummer RJ. Serotonergic modulators in the treatment of irritable bowel syndrome - influence on psychiatric and gastrointestinal symptoms. Aliment Pharmacol Ther 2003;17:43-51.

8. Atkinson W, Lockhart S, Whorwell PJ, Keevil B, Houghton LA. Altered 5-hydroxytryptamine signaling in patients with constipation- and diarrhea-predominant irritable bowel syndrome Gastroenterology 2006;130:34-43.

9. Yeo A, Boyd $P$, Lumsden $S$, Saunders $T$, Handley A, Stubbins M, Knaggs $A$, Asquith $S$, Taylor I, Bahari B, Crocker N, Rallan R, Varsani S, Montgomery D, Alpers DH, Dukes GE, Purvis I, Hicks GA. Association between a functional polymorphism in the serotonin transporter gene and diarrhoea predominant irritable bowel syndrome in women. Gut 2004; 53:1452-1458.

10. Heitkemper M, Jarrett M, Cain K, Shaver J, Bond E, Woods NF, Walker E. Increased urine catecholamines and cortisol in women with irritable bowel syndrome. Am J Gastroenterol 1996;91:906-913.

11. Patacchioli FR, Angelucci $L$, Dellerba G, Monnazzi $P$, Leri $O$. Actual stress, psychopathology and salivary cortisol levels in the irritable bowel syndrome (IBS). J Endocrinol Invest 2001; 24:173-177.

12. Leonard $\mathrm{BE}$. The HPA and immune axes in stress: the involvement of the serotonergic system. Eur Psychiatry 2005;20 Suppl 3:S302-306.

13. Dinan TG, Quigley EM, Ahmed $S M$, Scully $P$, O'Brien $S$, O'Mahony $L$, O'Mahony $S$, Shanahan F, Keeling PW. Hypothalamic-pituitary-gut axis dysregulation in irritable bowel syndrome: plasma cytokines as a potential biomarker? Gastroenterology 2006;130:304-311.

14. Chadwick VS, Chen W, Shu D, Paulus B, Bethwaite P, Tie A, Wilson I. Activation of the mucosal immune system in irritable bowel syndrome. Gastroenterology 2002;122: 1778-1783.

15. Dunlop SP, Jenkins $D$, Neal KR, Spiller RC. Relative importance of enterochromaffin cell hyperplasia, anxiety, and depression in postinfectious IBS. Gastroenterology 2003;125: 16511659.

16. Steens J, Van Der Schaar PJ, Penning C, Brussee J, Masclee AA. Compliance, tone and sensitivity of the rectum in different subtypes of irritable bowel syndrome. Neurogastroenterol Motil 2002;14:241-247.

17. Zar S, Benson MJ, Kumar D. Rectal afferent hypersensitivity and compliance in irritable bowel syndrome: differences between diarrhoeampredominant and constipation-predominant subgroups. Eur J Gastroenterol Hepatol 2006;18:151-158.

18. Kilkens TO, Honig A, Fekkes D, Brummer RJ. The effects of an acute serotonergic challenge on brain-gut responses in irritable bowel syndrome patients and controls. Aliment Pharmacol Ther $2005 ; 22: 865-874$ 
$158 \mid$ Chapter 8

19. Kilkens TO, Honig A, van Nieuwenhoven MA, Riedel WJ, Brummer RJ. Acute tryptophan depletion affects brain-gut responses in irritable bowel syndrome patients and controls. Gut 2004;53:1794-1800.

20. Drossman DA, Heaton K, Irvine EJ, Muller-Lissner S. Functional bowel disorders and functional abdominal pain. In: Drossman DA, Corazziari E, Talley N, Thompson WG, Whitehead WE, editors. The functional gastrointestinal disorders. Diagnosis, pathophysiology and treatment: A multinational consensus. McLean, VA: Degnon Associates; 2000:351-398.

21. Ledochowski $M$, Sperner-Unterweger $B$, Fuchs $D$. Lactose malabsorption is associated with early signs of mental depression in females: a preliminary report. Dig Dis Sci 1998:43:2513-2517.

22. Hamilton M. Development of a rating scale for primary depressive illness. $\mathrm{Br} \mathrm{J}$ Soc Clin Psychol 1967;6:278-296.

23. Arrindell WA, Ettema JHM. SCL-90. Een multidimensionale psychopathologie indicator [SCL90. A multidimensional indicator of psychopathology]. In. Lisse, The Netherlands: Swets \& Zeitlinger; 1986.

24. Mykletun $A$, Stordal E, Dahl AA. Hospital Anxiety and Depression (HAD) scale: factor structure, item analyses and internal consistency in a large population. $\mathrm{Br} J$ Psychiatry 2001; 179: $540-544$

25. Lagier $E$, Delvaux M, Vellas B, Fioramonti J, Bueno L, Albarede JL, Frexinos J. Influence of age on rectal tone and sensitivity to distension in healthy subjects. Neurogastroenterol Motil 1999:11:101-107.

26. Tanriverdi F, Silveira LF, MacColl GS, Bouloux PM. The hypothalamic-pituitary-gonadal axis: immune function and autoimmunity. J Endocrinol 2003;176:293-304.

27. Houghton LA, Lea R, Jackson N, Whorwell PJ. The menstrual cycle affects rectal sensitivity in patients with irritable bowel syndrome but not healthy volunteers. Gut 2002;50:471-474.

28. Maes M, Scharpe S, Verkerk R, D'Hondt P, Peeters D, Cosyns P, Thompson P, De Meyer F, Wauters A, Neels $H$. Seasonal variation in plasma L-tryptophan availability in healthy volunteers. Relationships to violent suicide occurrence. Arch Gen Psychiatry 1995;52: 937-946.

29. Stronks DL, Tulen JH, Verheij R, Boomsma F, Fekkes D, Pepplinkhuizen L, Mantel GW, Passchier J. Serotonergic, catecholaminergic, and cardiovascular reactions to mental stress in female migraine patients. A controlled study. Headache 1998;38:270-280.

30. Fekkes D, Timmerman L, Pepplinkhuizen L. Effects of clomipramine on plasma amino acids and serotonergic parameters in panic disorder and depression. Eur Neuropsychopharmacol 1997;7:235-239.

31. Schins A, Hamulyak K, Scharpe S, Lousberg R, Van Melle J, Crijns H, Honig A. Whole blood serotonin and platelet activation in depressed post-myocardial infarction patients. Life Sci 2004;76:637-650.

32. Vining RF, McGinley RA. The measurement of hormones in saliva: possibilities and pitfalls. $J$ Steroid Biochem 1987;27:81-94.

33. Ansseau $M$, Sulon J, Doumont $A$, Cerfontaine JL, Legros JJ, Sodoyez JC, Demey-Ponsart E. Use of saliva cortisol in the dexamethasone suppression test. Psychiatry Res 1984;13: 203-211.

34. Hair JF. Multivariate data analysis. New Yersey: Prentice-Hall, Inc. A Simon \& Schuster Company; 1998.

35. Pata $C$, Erdal ME, Derici $E$, Yazar A, Kanik A, Ulu $O$. Serotonin transporter gene polymorphism in irritable bowel syndrome. Am J Gastroenterol 2002;97:1780-1784.

36. Zhu CB, Blakely RD, Hewlett WA. The Proinflammatory Cytokines Interleukin-1beta and Tumor Necrosis Factor-Alpha Activate Serotonin Transporters. Neuropsychopharmacology 2006.

37. Linden DR, Foley KF, McQuoid C, Simpson J, Sharkey KA, Mawe GM. Serotonin transporter function and expression are reduced in mice with TNBS-induced colitis. Neurogastroenterol Motil 2005;17:565-574.

38. Mawe GM, Ciolino A, Foley KF. Inflammatory cytokines decrease serotonin transporter function in colonic epithelial cells. Neurogastroenterol Motil 2005;17:613. 
Rectal compliance and neurohormonal-immune signalling $\mid 157$

39. Simren M, Simms L, D'Souza D, Abrahamsson H, Bjornsson ES. Lipid-induced colonic hypersensitivity in irritable bowel syndrome: the role of 5-HT3 receptors. Aliment Pharmacol Ther 2003;17:279-287.

40. Thumshirn M, Coulie B, Camilleri M, Zinsmeister AR, Burton DD, Van Dyke C. Effects of alosetron on gastrointestinal transit time and rectal sensation in patients with irritable bowel syndrome. Aliment Pharmacol Ther 2000;14:869-878.

41. Zighelboim J, Talley NJ, Phillips SF, Harmsen WS, Zinsmeister AR. Visceral perception in irritable bowel syndrome. Rectal and gastric responses to distension and serotonin type 3 antagonism. Dig Dis Sci 1995;40:819-827.

42. Tack J, Broekaert D, Corsetti M, Fischler B, Janssens J. Influence of acute serotonin reuptake inhibition on colonic sensorimotor function in man. Aliment Pharmacol Ther 2006; 23:265-274.

43. Khan WI, Collins SM. Gut motor function: immunological control in enteric infection and inflammation. Clin Exp Immunol 2006;143:389-397.

44. Yang S, Zhang L. Glucocorticoids and vascular reactivity. Curr Vasc Pharmacol 2004;2: 1-12.

45. Posserud I, Agerforz P, Ekman R, Bjornsson ES, Abrahamsson H, Simren M. Altered visceral perceptual and neuroendocrine response in patients with irritable bowel syndrome during mental stress. Gut 2004;53:1102-1108.

46. Nicolson $N$, Storms $C$, Ponds $R$, Sulon J. Salivary cortisol levels and stress reactivity in human aging. J Gerontol A Biol Sci Med Sci 1997;52:M68-75.

47. Elsenbruch $S$, Orr WC. Diarrhea- and constipation-predominant IBS patients differ in postprandial autonomic and cortisol responses. Am J Gastroenterol 2001;96:460-466.

48. Paffen MLJE, Kilkens TOC, Honig A, Brummer R-JM, Van Nieuwenhoven MA. Does a rectal barostatprocedure induce stress in IBS patients and healthy controls? Eur J Gastroenterol Hepatol 2003;15:A38.

49. Lucas A, Holtmann G, Gerken G, Pietsch A, Braun-Lang U, Gilani K, Strassburger K, Gesing $S$, Janssen $O E$, Kavelaars $A$, Heijnen $C J$, Schedlowski M, Elsenbruch S. Visceral pain and public speaking stress: neuroendocrine and immune cell responses in healthy subjects. Brain Behav Immun 2006;20:49-56.

50. Yatham LN, Steiner M. Neuroendocrine probes of serotonergic function: a critical review. Life Sci 1993;53:447-463.

51. Won KJ, Suzuki T, Hori M, Ozaki H. Motility disorder in experimentally obstructed intestine: relationship between muscularis inflammation and disruption of the ICC network. Neurogastroenterol Motil 2006;18:53-61.

52. van der Veek PP, van den Berg $M$, de Kroon YE, Verspaget HW, Masclee AA. Role of tumor necrosis factor-alpha and interleukin-10 gene polymorphisms in irritable bowel syndrome. Am J Gastroenterol 2005;100:2510-2516.

53. Elsenbruch S, Holtmann G, Oezcan D, Lysson A, Janssen O, Goebel MU, Schedlowski M. Are there alterations of neuroendocrine and cellular immune responses to nutrients in women with irritable bowel syndrome? Am J Gastroenterol 2004;99:703-710.

54. Elenkov IJ, lezzoni DG, Daly A, Harris AG, Chrousos GP. Cytokine dysregulation, inflammation and well-being. Neuroimmunomodulation 2005;12:255-269.

55. Wrona D. Neural-immune interactions: An integrative view of the bidirectional relationship between the brain and immune systems. J Neuroimmunol 2006;172:38-58. 


\section{Chapter 9}

General discussion 


\section{General discussion}

The primary aim of this thesis was to elucidate the involvement of serotonin in brain-gut interactions with relevance to the pathophysiology of diarrhoeapredominant irritable bowel syndrome (d-IBS). We reviewed literature regarding the role of serotonin (5-HT) in brain-gut signalling and effects of treatment through altering serotonergic signalling in IBS. We proposed a novel research framework (i.e paradigm) to further investigate the role of 5-HT in brain-gut (dys)regulation. This framework includes simultaneous assessment of various domains of the brain-gut axis and techniques to modify serotonergic activity. This research framework was applied by investigating effects of acute 5-HT modulation, using both the acute tryptophan depletion (ATD) method and citalopram challenge test, on brain-gut responses in d-lBS patients and healthy controls. In addition we investigated baseline differences in mediators of braingut signalling in d-IBS patients and healthy controls.

The main findings of our studies are integrated and discussed by addressing the following issues:

- To what extend do these results support the hypothesis of serotonergic involvement in brain-gut signalling with regard to IBS pathophysiology?

- To what extend are these results applicable and constitute valid foundations for IBS research, clinical practice and therapy?

- Which recommendations can be made regarding future IBS research?

\section{Serotonergic modulation paradigms and assessment of brain-gut responses.}

Acute tryptophan depletion increased urge scores specifically in the lower pressure range and increased pain scores in d-IBS patients and healthy controls. In addition, ATD altered cortical activation in healthy controls and induced a significant shift in affective memory bias towards a preferential loss of positive material. During ATD, 5-HIAA levels, the major metabolite of 5-HT, were decreased which strongly suggests that lowered 5-HT activity alters braingut responses. The citalopram challenge test increased adrenocorticotropic hormone and cortisol levels compared with placebo and improved affective memory performance due to a bias towards positive material without a significant effect on visceral perception or rectal compliance in d-IBS patients and controls. Recently, Tack et al. demonstrated that, in healthy subjects rather than in IBS subjects, acute i.v. citalopram administration induced an increase in colonic phasic contractility and occurrence of high-amplitude propagated contractions, increased colonic compliance and a suppression of colonic tonic response to a meal ${ }^{1,2}$. The same group reported that citalopram significantly 
162 $\mid$ Chapter 9

improved IBS symptoms including abdominal pain after six weeks treatment (three weeks $20 \mathrm{mg}$, three weeks $40 \mathrm{mg}$ ) in non-depressed IBS patients. This therapeutic effect was independent of effects on mood and colonic sensorimotor function ${ }^{2}$. A recent animal study in mice demonstrated that selective serotonin reuptake inhibition significantly altered $\mathrm{Gl}$ motility and sensitivity ${ }^{3}$. Apparently, findings in literature regarding the effects of 5-HT on visceral sensorimotor function are equivocal possibly due to a differential effect of acute versus chronic serotonergic modulation at organ level. The complexity of $5-\mathrm{HT}$ signalling is further demonstrated by observations that peristaltic activity can be augmented by slight 5-HT stimulation, whereas over-stimulation can lead to a decreased efficiency, as well as by observations that deletion of serotonin reuptake transporter (SERT) in transgenic mice leads to a state of alternating diarrhoea and constipation ${ }^{4,5}$. An important point of notice is that both ATD and citalopram did not differentially affect the patient or control group. Although a type II error cannot be excluded, these results suggest that IBS is more a functional aberration rather than characterized by structurally altered 5-HT signalling pathways. However, 5-HT action could contribute to symptoms associated with IBS.

The clinical application of our primary findings is challenging. Our hypothesized role of 5-HT in IBS-related symptom generation is generally supported. Translation of these results of acute serotonergic modulation to chronic modulation is warranted. In chapter 3 long-term serotonergic treatment influences both gastroenterological and psychiatric symptoms in IBS. From personal experiences at the MedPsychUnit (MPU) at the University Hospital Maastricht (an inpatient clinic for patients with complex symptomatology in whom somatic and psychiatric symptoms or disease overly) treatment of refractory IBS with citalopram and mirtazapine showed relief of IBS symptoms with acceptable side effects. Continuation of this complex but interesting area of investigation is certainly needed. The acute tryptophan depletion model might serve as a research model for IBS in healthy subjects. ATD as a model for IBS was first implicated in our functional magnetic resonance imaging (fMRI) study.

With regard to future research we have the following recommendations:

Design. Because all studies were based on cross-sectional data, temporal ordering of any association between brain-gut responses and serotonergic parameters cannot be established. As addressed in chapter 2, acute versus long-term effects of 5-HT modulation may differ both qualitatively and quantitatively. Application of standardized nutrient intake and external stressors might increase the probability to detect differences between IBS patients and healthy controls. In addition, inclusion of other domains of the brain-gut axis 
signalling, such as the autonomic nervous system and immune function, is advisable.

Currently, the role of affective dysregulation in IBS symptom generation remains unclear. Our data support that affective dysregulation is indeed an important mediator influencing brain-gut responses. It would be appealing to study IBS patients with and without affective dysregulation, patients with affective dysregulation without abdominal complaints, and healthy controls, respectively, in order to unravel whether $5-\mathrm{HT}$ signalling is only a surrogate marker of affective dysregulation or has an additive affective dysregulationindependent contribution to IBS symptom generation. Determination of symptom severity at the time of testing and dividing patients into normosensitive and hypersensitive patients would be useful, especially in larger patient groups ${ }^{6}$.

Visceral sensorimotor function. The barostat procedure was useful to detect differences between IBS patients and controls as well as the effects of ATD. Although often used in current literature we did not assess minimal distension pressure (MDP) in our barostat studies. Using the MDP might influence results especially in the low distension pressure range. In addition, our barostat protocol was not primarily designed to analyse rectal compliance. inclusion of a staircase protocol with $2-\mathrm{mmHg}$ increases should be considered in order to obtain a proper pressure-volume curve.

Cerebral cortical activity induced by perception of a stimulus represents two intertwined processes: 1) registration of the sensory signal and 2) perceptionrelated cognitive processing ${ }^{7}$. Sensory and attentional networks for somatic pain have been described by Peyron et al. using position emission tomography (PET). They demonstrated that the insular and somatosensory cortices mediate sensory-discriminative aspects of pain whereas thalamus, prefrontal cortex, posterior parietal cortex, and anterior cingulate cortex (ACC) mediate attentional aspects of pain. In particular the ACC was not found to be involved in intensity coding of pain but instead was involved in attentional mechanisms triggered by pain $^{8}$. We asked our subjects to rate their perception and intensity of urge and pain. Patients differed from controls especially with regard to (subjective) pain perception. Although fMRI may give some insight into mechanisms of perception, interpretation and quantification of areas of activation with regard to visceral perception is still difficult. There is little experience with the affective (unpleasant) dimension of visceral perception. As supported by our ATD and fMRI studies this might be independent from the intensity of perception, which might be of special relevance to IBS $^{9}$.

Mood and cognition. In contrast to our expectation, we did not demonstrate any differences in mood, using the profile of mood states (POMS), during both the ATD and citalopram challenges. Using the verbal learning tasks, however, memory effects were significantly associated with affective connotation of the 
words. We suggested that this is a form of (mood congruent) response bias, which may be a more sensitive central indicator of affective tendencies. Recently, children with recurrent abdominal pain (RAP) showed non-conscious attention to and conscious avoidance of threat-related words related to individual differences in symptom severity ${ }^{10}$. As explained in chapter 2 , it would be interesting to study the presence of conscious or even non-conscious response bias in IBS. This response bias might be the key point of action regarding the positive effects of hypnotherapy in $\mathrm{IBS}^{11}$.

\section{Lipid profile: a mediator of the brain-gut axis?}

It is unknown whether serotonergic metabolism is directly related to lipid metabolism and plasma lipid profile as we did not analyze the direct relationships between 5-HT parameters and lipid profile. IBS patients showed increased scores of affective dysregulation compared to healthy controls, while polyunsaturated fatty acids (PUFAs) profile and serum cholesterol levels were significantly associated with markers of affective dysregulation. However, in contrast to patients with affective disorders, there were no significant differences in PUFAs and cholesterol indices between IBS patients and healthy matched controls. Hence, it can only be speculated that the increased levels of affective dysregulation in the IBS group are related to aberrations in 5-HT signalling.

A direct causal relationship between intermediary metabolism and brain-gut symptom generation with regard to IBS pathophysiology is unlikely and would conflict with the concept of IBS being a disorder rather than a disease entity. The presence of an association between level of affective dysregulation and lipid profile in the total study population suggests, however, a potential role for lipid profile as a metabolic mediator or biomarker in the brain-gut interaction. Hence, further investigation of intermediary metabolism in IBS is warranted especially in relation to gut and systemic inflammation. Supplementation with $n-3$ PUFAs (as potential mood and inflammatory mediators) or consumption of a diet rich in n-3 PUFAs in patients with IBS and co-occurrence of affective dysregulation needs further study and evidence before valid recommendations can be made.

Serotonergic and immune pathways are of potential importance in order to elucidate mechanisms by which intermediary metabolism may influence braingut signalling. Future research should include intestinal biopsies of IBS patients in order to subdivide IBS patients into groups with and without signs of lowgrade intestinal inflammation, as well as parameters of 5-HT metabolism at both the $\mathrm{Gl}$ and systemic level. Furthermore, its relationships with biomarkers of lipid metabolism and affective dysregulation should be investigated. 


\section{Unravelling the black box: missing pieces?}

Chapter 8 shows that $d$-IBS patients demonstrated significantly increased platelet poor plasma (ppp) 5-HT levels compared to controls. We did not demonstrate differences in ppp 5-HT between patients and controls in chapter 5 and 7 , respectively, using smaller subject groups. At the time of developing the research paradigm for this thesis differences in ppp 5-HT between d-IBS and controls were unknown. Currently, increased ppp 5-HT in d-IBS and postinfectious patients, especially in a post-prandial state have been reported by other research groups and constitute the first direct evidence of serotonergic involvement in IBS pathophysiology ${ }^{12}$. Salivary cortisol levels, used as an indication of hypothalamic-pituitary-adrenal (HPA) axis activity, tended to be increased in d-IBS patients compared to controls. Failure to reach significance may be due to a type $\|$ error as serum levels of cortisol were significantly increased in d-IBS patients compared to controls (chapter 7). An overall group association was found between ppp 5-HT, salivary cortisol, and tumour necrosis factor-alpha (TNF- $\alpha$ ) levels respectively, with reduced rectal compliance. In addition, a positive association between ppp 5-HT and salivary cortisol levels was found. These findings not only support the role of serotonin in IBS pathophysiology but also confirm the connections of serotonergic signalling with other mediators of brain-gut function including neuroendocrine and immune mechanisms.

These findings form an interesting point of departure for future research. The clear associations between neurohormonal-immune parameters and rectal compliance in the entire study population suggest a direct biological association between these parameters, rather than the existence of a dominant disease modifying factor specific for IBS. It may be concluded that not only subtle aberrations in intermediary metabolism, as mentioned earlier, but also in neurohormonal-immune signalling may underlie brain-gut dysregulation in $\mathrm{d}$ IBS.

With regard to clinical practice and therapy, however, these current findings are too preliminary as valid point of actions. It may be hypothesized that subjects prone to develop post-infectious or d-IBS are hyperresponsive with regard to inflammatory responses, due to alterations in the neuro-hormonal immune system. Prolonged nutritional supplementation or high dietary intake of specific PUFAs rather than pharmacological supplements may be useful in the prevention of post-infectious and d-IBS symptoms.

Regarding future research more comprehensive assessment of immune status and responsiveness is necessary ${ }^{13}$. We assessed neuro-hormonal status and rectal compliance on the same test day. The presence of anticipatory anxiety, although potentially inherent to the pathophysiology of IBS, must be taken into 
$166 \mid$ Chapter 9

consideration ${ }^{14}$. Applications of specific challenges including stress paradigms and corticotropin-releasing hormone (CRH) infusion tests as well as nutritional modulation are valuable tools for evaluating neuro-hormonal immune responses. Although of potential interest, as mentioned in chapter 2, we did not include autonomic nervous system (ANS) measurements. It remains a challenge for the future to adequately integrate the diverse domains of the brain-gut axis into one research paradigm.

\section{Final conclusion}

This thesis supports the involvement of $5-H T$ in brain-gut responses. Direct evidence of 5-HT dysregulation in IBS is reflected by the increased ppp 5-HT levels in d-IBS compared to carefully matched healthy control subjects. Moreover, 5-HT influences specific brain-gut responses which are assumed to differentiate IBS patients from healthy controls. However, serotonergic "tone" is not directly related to brain-gut responses but potentially influenced by other domains of the brain-gut axis. Paradoxes remain such as the positive influence of citalopram administration on IBS symptoms and the presence of increased ppp 5-HT in $\mathrm{IBS}^{2,12}$. Currently it can even be disputed whether drug therapy altering $5-\mathrm{HT}$ signalling is targeting symptoms rather than disease modifying. Apparently, relationships between systemic, gastrointestinal, and central nervous system 5-HT metabolism as well as the influence of acute and chronic serotonergic modulation on each of these systems needs further elucidation.

To define disorders of unknown pathology represents a substantial change in thinking for doctors whose clinical training concentrates on "palpable evidence". Brain-gut function is clearly a complex matter and it remains a challenge for the future to adequately understand and integrate the various domains of the braingut axis. This requires input of knowledge not only from gastroenterological and psychiatric but also neuropsychological, radiological, immunological and neurophysiological expertise. A transdisciplinary approach is pivotal in medical education, clinical practice (translational medicine e.g. MPU) as well as in medical research in order to achieve breakthroughs and eventually provide better treatment for patients with functional gastrointestinal disorders. 


\section{References}

1. Tack J, Broekaert D, Corsetti M, Fischler B, Janssens J. Influence of acute serotonin reuptake inhibition on colonic sensorimotor function in man. Aliment Pharmacol Ther 2006; 23:265-274.

2. Tack J, Broekaert D, Fischler B, Van Oudenhove L, Gevers A, Janssens J. A controlled crossover study of the selective serotonin reuptake inhibitor citalopram in irritable bowel syndrome. Gut 2006;55:1095-1103.

3. Coates MD, Johnson AC, Greenwood-Van Meerveld B, Mawe GM. Effects of serotonin transporter inhibition on gastrointestinal motility and colonic sensitivity in the mouse. Neurogastroenterol Motil 2006;18:464-471.

4. Mawe GM, Coates MD, Moses PL. Review article: intestinal serotonin signaling in irritable bowel syndrome. Aliment Pharmacol Ther 2006;23:1067-1076.

5. Chen JJ, Li Z, Pan $H$, Murphy DL, Tamir $H$, Koepsell $H$, Gershon MD. Maintenance of serotonin in the intestinal mucosa and ganglia of mice that lack the high-affinity serotonin transporter: Abnormal intestinal motility and the expression of cation transporters. $J$ Neurosci 2001:21:6348-6361.

6. Zar S, Benson MJ, Kumar D. Rectal afferent hypersensitivity and compliance in irritable bowel syndrome: differences between diarrhoea-predominant and constipation-predominant subgroups. Eur J Gastroenterol Hepatol 2006;18:151-158.

7. Sidhu $H$, Kern $M$, Shaker R. Absence of increasing cortical fMRI activity volume in response to increasing visceral stimulation in IBS patients. Am J Physiol Gastrointest Liver Physiol 2004;287:G425-435.

8. Peyron R, Garcia-Larrea L, Gregoire MC, Costes N, Convers $P$, Lavenne F, Mauguiere F, Michel D, Laurent $B$. Haemodynamic brain responses to acute pain in humans: sensory and attentional networks. Brain 1999;122:1765-1780.

9. Verne GN, Himes NC, Robinson ME, Gopinath KS, Briggs RW, Crosson B, Price DD. Central representation of visceral and cutaneous hypersensitivity in the irritable bowel syndrome. Pain 2003;103:99-110.

10. Boyer MC, Compas BE, Stanger C, Colletti RB, Konik BS, Morrow SB, Thomsen AH. Attentional biases to pain and social threat in children with recurrent abdominal pain. $J$ Pediatr Psychol 2006;31:209-220.

11. Whorwell PJ. Review article: The history of hypnotherapy and its role in the irritable bowel syndrome. Aliment Pharmacol Ther 2005;22:1061-1067.

12. Atkinson W, Lockhart S, Whorwell PJ, Keevil B, Houghton LA. Altered 5-hydroxytryptamine signaling in patients with constipation- and diarrhea-predominant irritable bowel syndrome. Gastroenterology 2006;130:34-43.

13. Dinan TG, Quigley EM, Ahmed SM, Scully P, O'Brien S, O'Mahony L, O'Mahony S, Shanahan F, Keeling PW. Hypothalamic-pituitary-gut axis dysregulation in irritable bowel syndrome: plasma cytokines as a potential biomarker? Gastroenterology 2006;130:304-311.

14. Paffen MLJE, Kilkens TOC, Honig A, Brummer R-JM, Van Nieuwenhoven MA. Does a recta! barostatprocedure induce stress in IBS patients and healthy controls? Eur $\mathrm{J}$ Gastroenterol Hepatol 2003;15:A38. 
Summary 


\section{Summary}

The irritable bowel syndrome (IBS) is a common functional gastrointestinal disorder (FGID) characterized by lower gastrointestinal (GI) tract symptoms that are conventionally not explained by structural abnormalities, infection, or metabolic changes. Although the pathophysiology of IBS is incompletely understood, IBS is now generally considered as a disorder primarily involving the brain-gut axis i.e. the bidirectional communication pathways connecting the enteric nervous system (ENS), the central nervous system (CNS), the autonomic nervous system (ANS), neuroendocrine centres, and the immune system, respectively. Serotonin is a biogenic amine that functions as a neurotransmitter and paracrine signalling molecule. Serotonin $(5-H T)$ is regarded as one of the key denominators involved in brain-gut interactions and signalling. The involvement of serotonin in brain-gut signalling as part of diarrhoea-predominant (d)-IBS pathophysiology was examined by application of a new research paradigm.

Chapter 1 is a general introduction to this thesis; it describes the (patho)physiological background of IBS, the brain-gut concept and the hypothesized pivotal role of serotonin in brain-gut signalling. In addition, the general aim and the outline of this thesis are presented.

Chapter 2 provides a framework for a new research paradigm to investigate brain-gut function and the potential role for serotonin in brain-gut signalling. We specifically focus on the assessment of integrated brain-gut function and serotonergic modulation techniques including the acute tryptophan depletion (ATD) method and the citalopram (SSRI) challenge test in healthy subjects in general and in d-IBS patients in particular. This paradigm includes several available biomarkers of brain-gut function including biomarkers at "end-organ" level i.e. at brain and gut level and mediators of brain-gut signalling including autonomic nervous system, intermediary metabolism, neuroendocrine-immune, and serotonergic systems, respectively. Serotonergic modulation of the braingut axis by these interventions and subsequent assessment of integrated braingut function offers the possibility to gain further insight into the role of serotonergic regulation and brain-gut function in functional gastrointestinal disorders. We concluded that the multi-component nature of IBS pathophysiology should be taken into consideration in order to achieve breakthroughs in pharmacological and nutritional interventions in IBS.

In order to elucidate potential differential effects of 5-HT modulation on IBS symptom generation Chapter 3 describes a standardized qualitative analysis of studies investigating the influence of 5-HT modulators on both gastrointestinal 
and psychiatric symptoms in IBS. Eleven studies fulfilled the entry criteria of which six scored above 55 points (an adequate quality of the paper). An association between gastroenterological and psychiatric changes was present in five of these six studies. We concluded that the results support the role of serotonin in the association between gastroenterological and psychiatric symptoms in IBS.

Irritable bowel syndrome is characterized by a high co-occurrence of affective dysregulation. Affective disorders have been associated with specific aberrations in intermediary metabolism characterized by alterations of the profiles of polyunsaturated fatty acids (PUFAs) in plasma phospholipids and serum cholesterol. If metabolic aberrations occur in IBS and if these aberrations show a high similarity with those that are present in patients with affective disorders, this would support the existence of shared pathophysiological routes. In Chapter 4 a case-control study is presented on the fatty acid profile of plasma phospholipids and cholesterol in association with affective dysregulation in $23 \mathrm{~d}$-IBS patients and 23 healthy matched controls. As expected, the level of affective dysregulation was higher in IBS compared with controls. PUFA and cholesterol profile did not differ significantly between subject groups. However, PUFA and cholesterol profiles were significantly associated with the level of affective dysregulation. Hence, we concluded that these results warrant further studies regarding the role of PUFA and cholesterol status as a pathophysiological denominator or biomarker in the co-occurrence of affective dysregulation and functional gastrointestinal disorders.

Chapter 5 describes the results of the first application of our new research paradigm and shows the effects of lowered serotonergic activity using the ATD method on visceral urge and pain perception during rectal distensions using a barostat procedure, on affective memory performance, and mood in fourteen dIBS patients and fourteen matched controls. ATD was associated with significantly increased urge scores, specifically in the lower pressure range, and overall increased pain scores. ATD induced a significant shift in affective memory bias towards preferential loss of positive material but no significant changes in mood appeared. ATD did not differentially affect the patient and control group. We concluded that acute serotonergic modulation by ATD affects visceral perception as well as cognition in d-IBS patients and controls.

Chapter 6 describes the effect of ATD on cortical activation during painful and non-painful rectal distension in twelve healthy females. This study was performed according to a placebo-controlled study design. Functional magnetic resonance imaging (fMRI) sessions were performed using block-related rectal barostat stimuli, interleaved with rest intervals. Painful rectal distension resulted 
in activation of the right insula and the right inferior frontal cortex, whereas nonpainful distension resulted in activation of the right insula, the right anterior cingulate cortex (ACC) and the right inferior and left orbitofrontal cortex. During ATD painful distension induced more activation in the left ACC, the left superior frontal cortex and right insula and putamen and non-painful distension resulted in more activation of the left putamen. We concluded that ATD leads to a shift from primary visceral pain perception towards a more affective or emotional experience of the visceral pain sensation.

The effects of an acutely increased serotonergic activity, using a $20 \mathrm{mg}$ intravenous citalopram challenge test (selective serotonin reuptake inhibitor), on visceral perception in response to rectal distensions, affective memory performance, mood, and neuroendocrine response (adrenocorticotropic hormone, cortisol, prolactin) in fourteen d-IBS patients and fourteen matched controls are described in Chapter 7. Visceral perception did not significantly differ between the citalopram and placebo condition. However, citalopram administration improved affective memory performance because of a bias towards positive material without significant changes in mood. Citalopram significantly increased plasma serotonin, adrenocorticotropic hormone and cortisol levels compared with placebo. Citalopram did not differentially affect the patient and control group. We concluded that acutely increased serotonergic activity influences neuroendocrine responses and cognition in dIBS patients and controls without a significant effect on visceral perception.

In Chapter 8 differences in rectal compliance (pressure-volume relationship) and its relationship to biochemical parameters of serotonergic metabolism (platelet poor plasma 5-hydroxytryptamine (ppp 5-HT), its metabolite 5-hydroxyindole acetic acid (5-HIAA), 5-HT turnover (5-HIAA:5-HT), platelet 5-HT, hypothalamic-pituitary-adrenal (HPA) axis (salivary cortisol) and immune activation (serum levels of the proinflammatory cytokine TNF- $\alpha$ ), respectively, in 22 patients with d-IBS and 22 healthy controls were assessed. The d-IBS patients showed significantly increased ppp 5-HT levels, decreased 5-HIAA levels, decreased 5-HT turnover and reduced rectal compliance compared to controls. The levels of ppp 5-HT, cortisol and TNF- $\alpha$ correlated significantly with reduced rectal compliance. We concluded that reduced rectal compliance in $\mathrm{d}$-IBS is significantly related to alterations in neurohormonal and immune signalling.

In Chapter 9, the results of our studies are interpreted and discussed by addressing i) to what extend our results support the general concept of serotonergic involvement in brain-gut signalling with regard to IBS pathophysiology and ii) to what extend the results are applicable and constitute 
valid foundations for IBS research, clinical practice and therapy and iii) which recommendations can be made regarding future IBS research. Overall, this thesis supports the involvement of 5-HT in brain-gut responses. The brain-gut axis is a complex matter and a transdisciplinary approach is pivotal for the future to adequately understand the role of serotonin in brain-gut signalling and to integrate the various domains of the brain-gut axis in functional gastrointestinal research and treatment. 
Samenvatting 
\begin{tabular}{l|l} 
Samenvatting & 177
\end{tabular}

\section{Samenvatting}

Het prikkelbare darm syndroom (PDS) is een veel voorkomende functionele maag-darmaandoening (FGID) die wordt gekarakteriseerd door klachten van de lage tractus digestivus die niet kunnen worden verklaard door structurele afwijkingen, infecties of metabole afwijkingen. Hoewel de pathofysiologie van PDS maar ten dele is opgehelderd, wordt PDS tegenwoordig beschouwd als een aandoening van de hersen-darm as. Deze as verbindt het maagdarmzenuwstelsel, het centraal zenuwstelsel, het autonome zenuwstelsel, de neuroendocriene centra en het immuunsysteem. Serotonine $(5-\mathrm{HT})$ wordt beschouwd als een van de voornaamste neurotransmitters die de hersen-darm as reguleert. De rol van serotonine in de hersen-darm interactie en regulatie bij diarree-predominante (d)-PDS en gezonde personen werd onderzocht met een nieuw onderzoeks-paradigma.

Hoofdstuk 1 is een algemene introductie van dit proefschrift waarin de (patho)fysiologische achtergronden van PDS, het concept van de hersen-darm interactie en de veronderstelde cruciale rol van serotonine in de hersen-darm as worden beschreven. Verder wordt de hoofddoelstelling en een kort overzicht van dit proefschrift gepresenteerd.

Hoofdstuk 2 toont een raamwerk van een nieuw onderzoeksparadigma om de hersen-darm functie en de potentiële rol van serotonine in hersen-darm interactie te onderzoeken. De specifieke focus ligt op het meten van geïntegreerde hersen-darm functie en serotonerge modulatie technieken, te weten de acute tryptofaan depletie methode (ATD) en de citalopram challenge test bij gezonde vrijwilligers in het algemeen en bij mensen met PDS in het bijzonder. Er werd gekeken naar biomarkers van hersen-darm functie op "eindorgaan" nivo (hersenen en darm) en tevens naar mediatoren van hersen-darm signalering die betrekking hebben op het autonoom zenuwstelsel, het intermediair metabolisme en het neuroendocrien-immuun- en serotonerge systeem. Serotonerge modulatie van de hersen-darm as met behulp van deze interventies en het meten van geïntegreerde hersen-darm functie leidt tot meer inzicht in functionele maag-darm aandoeningen. We concludeerden dat we rekening moeten houden met het multifactoriële aspect van de pathofysiologie van PDS om doorbraken te realiseren in de behandeling van PDS.

Om de potentiele differentiële effecten van 5-HT modulatie op het onstaan van klachten bij PDS te verhelderen wordt in Hoofdstuk 3 een kwalitatieve analyse van studies gepresenteerd die de invloed van 5-HT modulatoren op zowel gastrointestinale als psychiatrische klachten beschrijft bij PDS. Elf studies voldeden aan de inclusie criteria waarvan er zes een score behaalden hoger 
dan 55 punten (hetgeen een voldoende kwaliteit van het artikel aangeeft). Een verband tussen verandering van gastroenterologische en psychiatrische klachten was aanwezig bij vijf van deze zes studies. We concludeerden dat de resultaten de rol van serotonine bij gastroenterologische en psychiatrische klachten bij PDS ondersteunen.

PDS wordt gekaraktariseerd door een hoge co-morbiditeit met affectieve dysregulatie. Deze stemmingsstoornissen zijn geassocieerd met specifieke afwijkingen in het intermediar metabolisme. Het wordt gekenmerkt door veranderingen in het profiel van meervoudig onverzadigde vetzuren (PUFAs) in plasma fosfolipiden alsook serum cholesterol. Indien metabole afwijkingen aanwezig zijn bij PDS die lijken op de afwijkingen bij patiënten met stemmingsstoornissen dan zou dit het bestaan van gezamelijke pathofysiologische routes ondersteunen. In Hoofdstuk 4 wordt een patiëntcontrole studie beschreven die het verband tussen het vetzuurprofiel van plasma fosfolipiden en cholesterol en de aanwezigheid van affectieve dysregulatie in 23 d-PDS patiënten en 23 gezonde gematchte controle personen onderzocht. Zoals verwacht was de mate van affectieve dysregulatie bij patiënten met PDS hoger dan die van gezonde controles. Het PUFA en cholesterol profiel verschilde niet significant tussen de patiënten en de controle groep. Echter, het PUFA en cholesterol profiel was significant geassocieerd met de mate van affectieve dysregulatie. Deze resultaten rechtvaardigen verder onderzoek naar de rol van PUFA en cholesterol status als een pathofysiologisch kenmerk van het samen optreden van affectieve dysregulatie en functionele maag-darm aandoeningen.

In Hoofdstuk 5 worden resultaten van ons nieuwe onderzoeksparadigma beschreven. Dit betreft de effecten van een verlaagde serotonerge activiteit middels de acute tryptofaan depletie techniek (ATD) op aandrang en pijn (viscerale) perceptie tijdens het opblazen van een ballon in de endeldarm (rectale distensies) met behulp van een barostat procedure op (affectieve) geheugen prestatie en stemming bij veertien d-PDS patiënten en veertien gematchte controle personen. Het toepassen van ATD ging gepaard met significant verhoogde aandrang scores speficiek bij lage distensie drukken en algemeen verhoogde pijnperceptie. ATD ging gepaard met een verslechtering van het geheugen, die specifiek was voor woorden met een positieve betekenis. ATD had geen significant effect op de stemming en de effecten van ATD verschilden niet tussen de patiënt- en de controle groep. We concludeerden dat acute serotonerge modulatie met behulp van ATD zowel de viscerale perceptie als cognitie beïnvloedt. 
In Hoofdstuk 6 worden de effecten van ATD op corticale activatie tijdens pijnlijke en niet-pijnlijke rectale distensies beschreven bij twaalf gezonde vrouwelijke personen in een placebo-gecontroleerde studie. Functionele magnetische resonantie-imaging (fMRI) metingen werden uitgevoerd tijdens blok-gerelateerde barostat stimuli met intervallen van rust. Pijnlijke rectale distensies veroorzaakten activatie van de rechter insula en de rechter inferieure frontale cortex en niet-pijnlijke distensies resulteerden in activatie van de rechter insula en de rechter anterieure cingulate cortex (ACC) en de rechter inferieure en linker orbitofrontale cortex. Tijdens ATD induceerden pijnlijke distensies meer activatie van de linker ACC, de linker superieure frontale cortex, de rechter insula en het putamen en niet-pijnlijke distensies resulteerden in meer activatie van het linker putamen. We concludeerden dat ATD een verschuiving veroorzaakt van primaire viscerale pijnperceptie naar een meer affectieve of emotionele ervaring van de viscerale pijn.

De effecten van een acuut verhoogde serotonerge activiteit middels de $20 \mathrm{mg}$ i.v. citalopram (selectieve serotonine heropname remmer) challenge test op viscerale perceptie tijdens rectale distensies, affectieve geheugen functie, stemming en neuro-endocriene respons (adrenocorticotroop hormoon, cortisol en prolactine) in veertien d-PDS patiënten en veertien gezonde gematchte controle proefpersonen worden beschreven in Hoofdstuk 7. De viscerale perceptie verschilde niet significant tussen de placebo en citalopram conditie. Echter, citalopram verbeterde de affectieve geheugenprestatie met een bias voor woorden met een positieve betekenis zonder dat er sprake was van een effect op de stemming. Citalopram verhoogde significant de plasma serotonine, adrenocorticotroop hormoon en cortisol waarden t.o.v. placebo. De effecten van citalopram verschilden niet tussen de patiënten en de controle groep. We concludeerden dat acuut verhoogde serotonerge activiteit de neuroendocrine respons en cognitie beïnvloedt bij d-PDS patiënten en gezonde controles zonder dat het een effect heeft op de viscerale perceptie.

In Hoofdstuk 8 worden verschillen in rectale compliantie (druk-volume relatie) en de relatie met biochemische parameters van het serotonerge metabolisme (bloedplaatjesarm plasma 5-hydroxytryptamine (ppp 5-HT), de metaboliet 5-hydroxyindole acetic acid (5-HIAA), 5-HT turnover (5-HIAA:5-HT), plaatjes $5-\mathrm{HT}$ ), van de HPA as (cortisol in speeksel) en van immuunactivatie (serum waardes van de proinflammatoire cytokine TNF- $\alpha$ ) bepaald in 22 patiënten met d-PDS en 22 gezonde controle personen. De d-PDS patiënten hadden significant verhoogde ppp 5-HT waarden, verlaagde 5-HIAA waarden, een verlaagde $5-\mathrm{HT}$ turnover en verlaagde rectale compliantie vergeleken met controles. De waarden van ppp 5-HT, cortisol en TNF- $\alpha$ hadden een significant verband met verlaagde rectale compliantie. We concludeerden dat verlaagde 
rectale compliantie in d-PDS significant is gerelateerd aan veranderingen in neurohormonale en immuun signalering.

In Hoofdstuk 9 worden de resultaten van onze studies geïnterpreteerd en bediscussieerd door het volgende aan de orde te stellen:

i) in hoeverre ondersteunen onze resultaten serotonerge betrokkenheid in hersen-darm signalering met betrekking tot de pathofysiologie van PDS, ii) in hoeverre deze resultaten toepasbaar zijn en een valide basis vormen voor PDS onderzoek, de klinische praktijk en behandeling en iii) welke aanbevelingen kunnen worden gemaakt met betrekking tot toekomstig PDS onderzoek.

De resultaten van dit proefschrift ondersteunen de serotonerge betrokkenheid van $5-H T$ in de hersen-darm as. De hersen-darm as is een complex concept en een transdisciplinaire benadering is cruciaal om in de toekomt de rol van serotonine beter te kunnen begrijpen en om de diverse domeinen van de hersen-darm as te kunnen integreren in het onderzoek bij en behandeling van functionele maag-darm aandoeningen. 
Nawoord 


\section{Nawoord}

Ik wil allen danken, die al dan niet bij naam genoemd, een bijdrage hebben geleverd aan de totstandkoming van dit proefschrift.

Een onweerstaanbare aantrekkingskracht van de begrippen sport en voeding afzonderlijk en de combinatie van beide in het bijzonder maakte dat ik tijdens mijn studie geneeskunde koos voor het "Onderzoeks Traject Geneeskunde". met als onderwerp "sport en voeding".

De persoon die ervoor heeft gezorgd dat het niet bij een kortstondige kennismaking bleef is Michiel van Nieuwenhoven: jij zag kans om in die éne week mijn wetenschappelijke wortels grond te geven. Hierdoor kon, maar vooral ook durfde ik enkele jaren later de beslissing te nemen om mijn studie te onderbreken voor de wetenschap.

Adriaan Honig en Robert-Jan Brummer: het project "Serotonerge mechanismen bij het prikkelbare darm syndroom" vloeide voort uit de nieuwe en uiterst interessante brug, die door jullie in de kliniek -in de vorm van de MedPsychUnit- was geslagen tussen de psychiatrie en de gastroenterologie. Ik hoop dat middels dit proefschrift het wetenschappelijk fundament hiervan is verstevigd.

Adriaan: na jouw vertrek naar Amsterdam zijn we elkaar de laatste jaren uit het oog verloren. Ik zal mij je grote rechtvaardigheidsgevoel en fanatieke inzet niet alleen voor het onderzoek, maar ook voor mij als onderzoeker, altijd bijzonder heugen.

Robert-Jan: tijdens mijn sollicitatiegesprek constateerde jij dat ik mogelijk licht neurotisch ben. Ik moet inmiddels constateren dat dit toch een typisch geval betreft van -de pot die... Het verwoorden van mijn gedachten verliep niet altijd even logisch. Maar als er iemand was die dit wel begreep en mijn manier van denken en redeneren heeft beïnvloed dan ben jij het wel. Je had het vaak erg druk, maar de momenten dat we er eens "goed voor gingen zitten" waren hoogtepunten tijdens mijn onderzoeksperiode.

I would like to thank all members of the reviewing committee: Prof. dr. J. Van Os, Prof. dr. A.A.M. Masclee, Prof. dr. M. Schemann, Dr. M. Simrén and Prof. dr. R.W. Stockbrügger, for their evaluation of the manuscript.

De oude en nieuwe opleider van het specialisme maag-darm-leverziekten: Beste Prof. Stockbrügger, hoewel ik nooit direct met $u$ heb samengewerkt bewonder ik $u$ op afstand. Een leven lang leren en gedreven blijven, het straalt van $u$ af. Beste Prof. Masclee, nu na mijn start in de kliniek, hoop ik mijn ambitie wetenschappelijk onderzoek te blijven doen, te gaan waarmaken. 
Mijn speciale dank gaat uit naar alle patiënten en vrijwilligers die de moeite hebben genomen om al die keren naar het ziekenhuis te komen om deel te nemen aan de soms niet al te plezierige en vaak lange testdagen. Dank aan mijn zus die na enige twijfel als proefpersoon heeft deelgenomen. Het feit dat je hierna zelf de eerste schreden op het pad van de wetenschap bent gaan zetten, bewijst maar weer eens dat een barostatprocedure of anders tryptofaan depletie wel degelijk de perceptie van zaken kan veranderen.

De studenten die hebben meegeholpen aan het onderzoek: jullie hulp, tomeloze inzet en betrokkenheid waren onontbeerlijk. Het was voor mij een groot compliment als jullie na jullie wetensschapsstage, vaak tegen jullie eigen verwachtingen in, constateerden dat het doen van onderzoek toch zeker zo saai niet is en eigenlijk gewoonweg leuk.

Medewerkers van de Universiteit Maastricht, het Academisch Ziekenhuis Maastricht en elders: jullie steun en vaak onbaatzuchtig advies waren en zijn voor mij een verademing.

De inmiddels door de jaren heen talloze directe collega's/kamergenoten: pagina's vol anekdotes zou ik kunnen schrijven... wat hebben we geklaagd maar vooral toch veel gelachen. $\mathrm{lk}$ hoop van harte jullie tijdens mijn promotie te mogen begroeten.

Mijn familie, vrienden, paranimfen Marcel en Wendy, ouders, Ans en Stan: ik ben bevoorrecht en blij met jullie! 
Curriculum vitae 


\section{Curriculum vitae}

Tessa Kilkens werd geboren op 3 november 1977 te Sittard. In 1996 behaalde zij haar VWO-diploma aan het Bisschoppelijk College te Echt en 4 jaar later haar doctoraal geneeskunde aan de Universiteit Maastricht cum laude. Zij onderbrak tijdelijk haar co-schappen om met haar onderzoek te starten bij de vakgroep Psychiatrie en Neuropsychologie resulterend in dit proefschrift. In maart 2006 behaalde ze haar basisartsdiploma en sinds augustus 2006 werkt zij als assistent in opleiding tot maag-darm-lever arts in het Academisch Ziekenhuis Maastricht. 